\title{
AN INTELLIGENT SUPPORT SYSTEM FOR THE ANALYSIS OF POWER SYSTEM TRANSIENTS
}

\author{
by \\ AWAD IBRAIK IBRAHIM \\ B.A.Sc. Garyouns University, Benghazi, Libya, 1989 \\ M.A.Sc. University of Waterloo, 1995 \\ A THESIS SUBMITTED IN PARTIAL FULFILLMENT OF \\ THE REQUIREMENTS FOR THE DEGREE OF \\ DOCTOR OF PHILOSOPHY \\ in \\ THE FACULTY OF GRADUATE STUDIES \\ DEPARTMENT OF ELECTRICAL \& COMPUTER ENGINEERING \\ We accept this thesis as conforming \\ to the required standard
}

THE UNIVERSITY OF BRITISH COLUMBIA

September 2000

(C) Awad Ibraik Ibrahim, 2000 
In presenting this thesis in partial fulfilment of the requirements for an advanced degree at the University of British Columbia, I agree that the Library shall make it freely available for reference and study. I further agree that permission for extensive copying of this thesis for scholarly purposes may be granted by the head of my department or by his or her representatives. It is understood that copying or publication of this thesis for financial gain shall not be allowed without my written permission.

Department of Electical \& Computur Eng.

The University of British Columbia Vancouver, Canada

Date $\operatorname{sep} 18,2000$ 


\begin{abstract}
Electromagnetic transients programs such as EMTP are extensively used for simulating fast transient effects in electric power systems. However, these programs are not easy to use for the following two reasons:
\end{abstract}

1. All of them require a high level of technical expertise to apply them properly.

2. Most of them are not user friendly.

This project proposes an intelligent system to support simulations with EMTP and similar programs. The work is composed of three parts; the first part presents the development of a prototype intelligent support system, the second part introduces the knowledge base for switching surge studies, and the third part describes the development of a new line model for the representation of short transmission lines and cables.

The intelligent support system is composed of three stages. First, the support system selects a base case from a case database, to be modified to meet the user's requirement. Second, the expert system checks the syntax and validity of the new case data. Finally, the EMTP solution is checked to see whether the chosen models are appropriate for the frequencies contained in the solution, for the degree of imbalance, and for other criteria. If they are not appropriate, better models will be recommended to the user.

The knowledge base for switching surge studies solves the problem of selecting the proper models for representing power system components in the EMTP. It helps in checking the validity of the data used to represent the simulated transient phenomena, and gives some suggestions to the user to correct his case data before simulation. It also helps in the evaluation of the results, using 
the knowledge of the phenomena being simulated. From this knowledge base, the idea of developing a new line model for short lines and cables evolved as one of the practical rules that is recommended for the proposed intelligent support system.

The new EMTP transmission line model for the representation of short overhead lines and cables will overcome the limitation of using a time step size not larger than the travel time. The error analysis for the short and open-circuit responses shows that the new line model has a filtering effect for higher frequencies. In a comparison with actual field test measurements, it is shown that the new line model is suitable for a reasonable representation of short overhead transmission lines and cables. 
$\begin{array}{lll}\text { Abstract } & \text { ii }\end{array}$

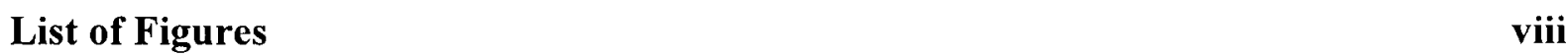

List of Tables $\quad$ xiii

Acknowledgment $\quad$ xiv

1 Introduction 1

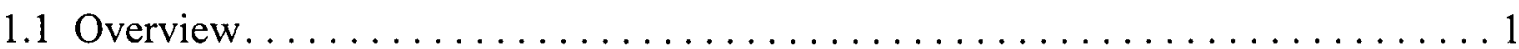

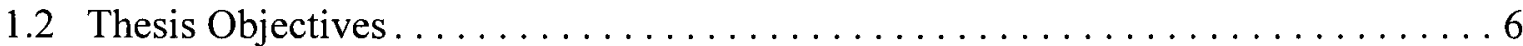

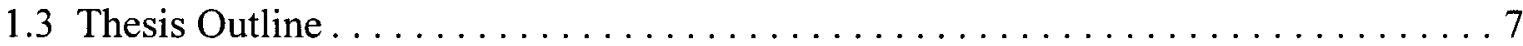

2 Intelligent Support System $\quad 9$

2.1 Introduction. . . . . . . . . . . . . . . . . . . . . . .

2.2 The Need for Using an Expert System Approach. . . . . . . . . . . . . . 11

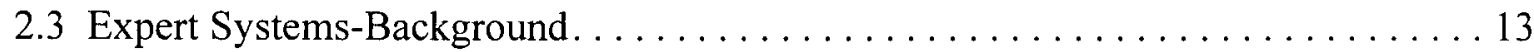

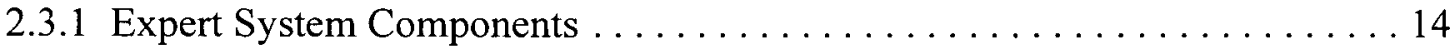

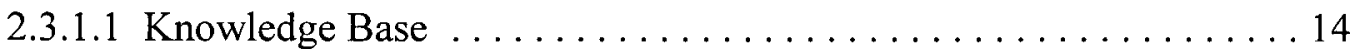

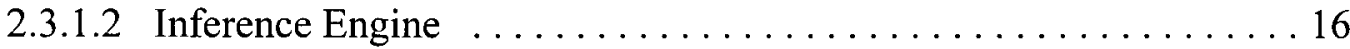

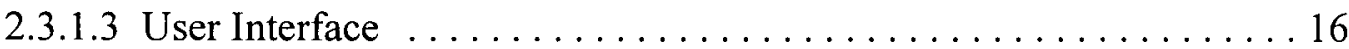

2.3.2 Applications of Expert Systems to Power Systems . . . . . . . . . . . 16

2.3.3 Integration of Expert Systems with Power Simulation Programs . . . . . . 18

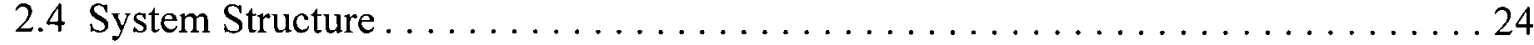

2.4.1 The Case Database. . . . . . . . . . . . . . . . . . . . . 26

2.4 .2 Data Validation ... . . . . . . . . . . . . . . . . . . . . . . . 27

2.4 .3 Results Evaluation . . . . . . . . . . . . . . . . . . . 27

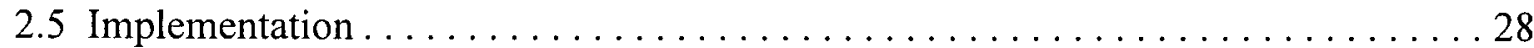

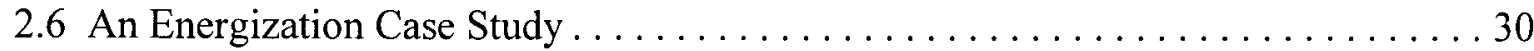

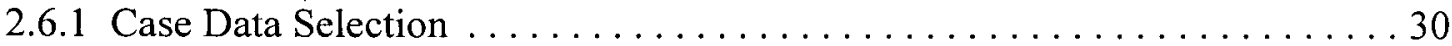

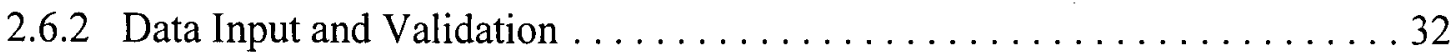


2.6.2.1 The Data Input Form $\ldots \ldots \ldots \ldots \ldots \ldots \ldots \ldots \ldots \ldots \ldots \ldots \ldots \ldots \ldots$

2.6.3 Rule-Based Data Validation System ....................... 34

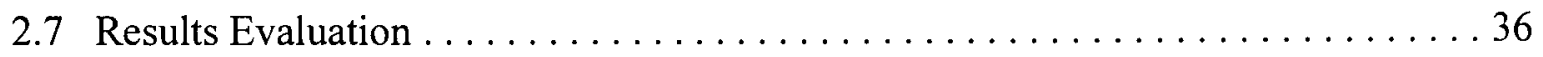

2.7.1 Characteristic Parameters ............................. 37

2.7.2 Step Size Variations . . . . . . . . . . . . . . . . . . . . . 37

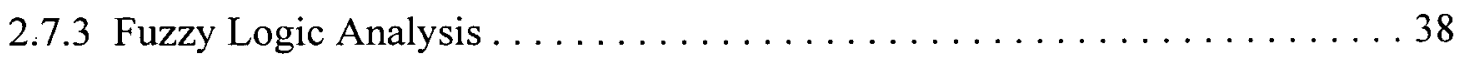

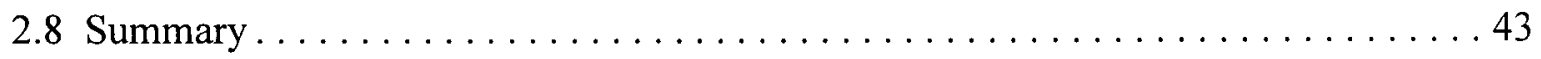

3 Switching Surge Transients $\quad 44$

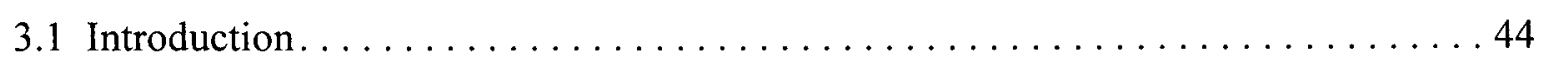

3.2 Switching Surge Transients-Background $\ldots \ldots \ldots \ldots \ldots \ldots \ldots \ldots \ldots \ldots$

3.2.1 Summary of Different Classes of Overvoltages .............. 45

3.2.2 Source and Characteristic of Switching Overvoltages ............. 46

3.2.3 Parameters Influencing Switching Overvoltages .............. 47

3.2.4 Switching Overvoltages in Closing and Reclosing Operations.......... 50

3.2.5 Definitions ................................... 51

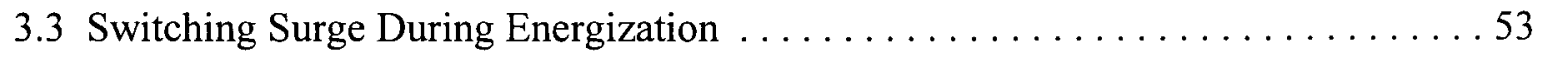

3.3.1 Network Configuration............................. 53

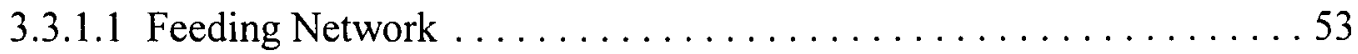

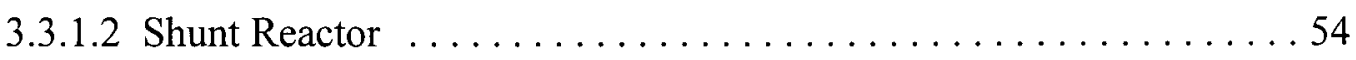

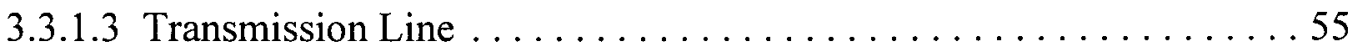

3.3.1.4 Circuit Breaker ........................... 55

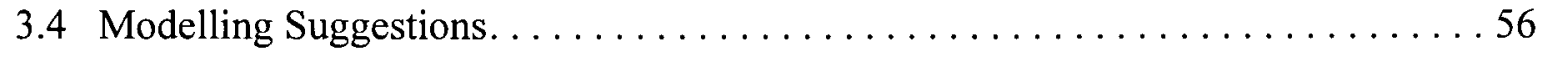

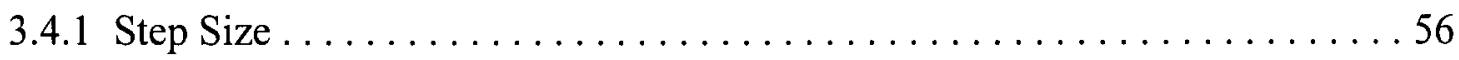

3.4 .2 Transmission Line Models . . . . . . . . . . . . . . . . . . . . . 59

3.4.2.1 The Constant Parameter Line Model $\ldots \ldots \ldots \ldots \ldots \ldots \ldots$

3.4.2.2 The Frequency Dependent Line Model ................... 61

3.4.2.3 Comparison Between the Two Line Models .............. 66 
3.4.2.4 The Single-Phase Energization Case Study . . . . . . . . . . . . 69

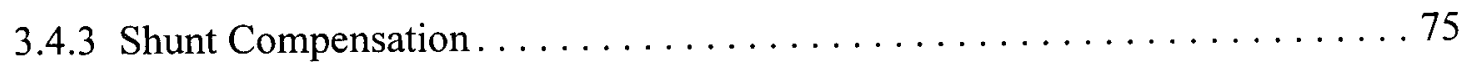

3.4 .4 Trapped Charges . . . . . . . . . . . . . . . . . . . 79

3.4 .5 Feeding Network. . . . . . . . . . . . . . . . . . . 81

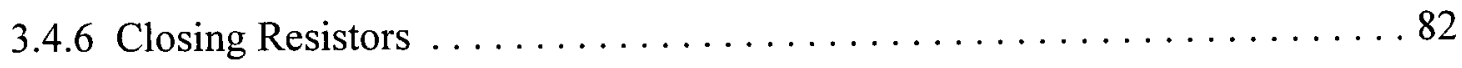

3.4 .7 Line Length. . . . . . . . . . . . . . . . . . . . . 85

3.4 .8 Closing Angle and Pole Span $\ldots \ldots \ldots \ldots \ldots \ldots \ldots \ldots \ldots \ldots \ldots \ldots \ldots \ldots \ldots \ldots$

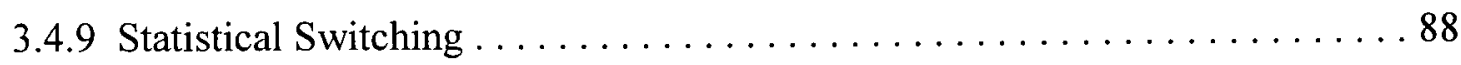

3.4.10 Derived Practical Rules from the Knowledge Base. . . . . . . . . . . . 89

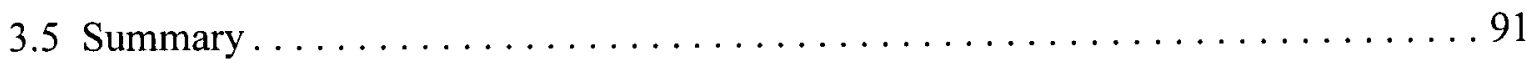

4 Transmission Line Model $\quad 93$

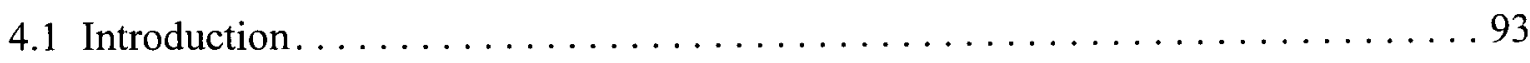

4.2 Transmission Line Models-Background . . . . . . . . . . . . . . . 94

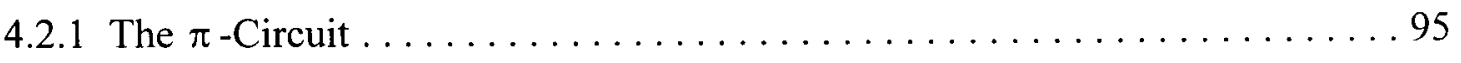

4.2.2 The Constant Parameter Line Model . . . . . . . . . . . . . . . . 96

4.2.3 The Frequency Dependent Line Model . . . . . . . . . . . . . 96

4.3 Transmission Line Model For Long Lines . . . . . . . . . . . . . . . . 96

4.4 The New Line Model For Short Lines . . . . . . . . . . . . . . . . . 97

4.5 Interpolation Error Analysis . . . . . . . . . . . . . . . . 100

4.6 The Lossy Line Model . . . . . . . . . . . . . . . . . . . 106

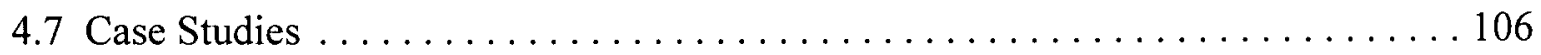

4.7.1 Transmission Line Energization . . . . . . . . . . . . . 106

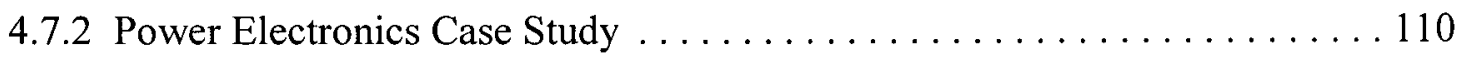

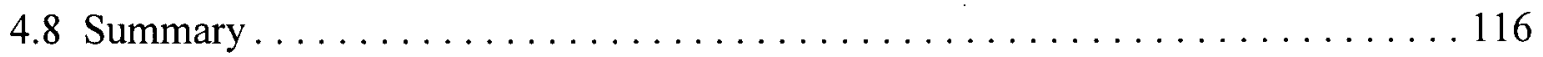

5 Conclusions and Future Work $\quad 117$

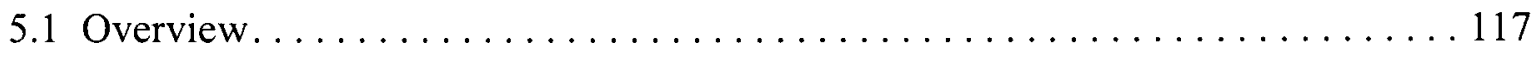




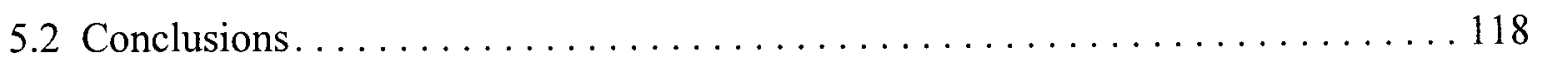

5.2 .1 The Prototype Intelligent Support System $\ldots \ldots \ldots \ldots \ldots \ldots \ldots \ldots$

5.2 .2 Switching Surge Overvoltages . . . . . . . . . . . . . . . 119

5.2 .3 New Transmission Line Model. . . . . . . . . . . . . . . . . . . . . . 119

5.3 Recommendations for Future Work . . . . . . . . . . . . . . . . . 119

$\begin{array}{ll}\text { Bibliography } & 121\end{array}$

$\begin{array}{ll}\text { A The Case Database } & 131\end{array}$

B EMTP Connec Subroutine for the New Line Model 152

$\begin{array}{lr}C \text { Interpolation Error Analysis } & 160\end{array}$

D Input \& Output Data Files for EMTP 173 


\section{List of Figures}

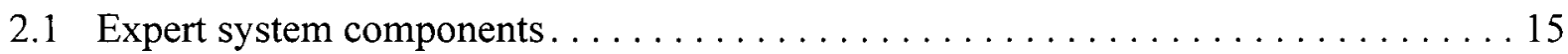

2.2 Structure of transient simulation support system $\ldots \ldots \ldots \ldots \ldots \ldots \ldots \ldots$

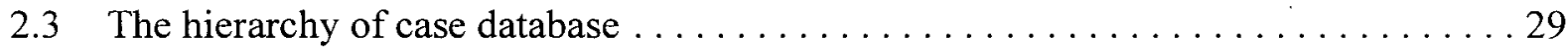

2.4 System configuration for a single-phase switching case study $\ldots \ldots \ldots \ldots \ldots$

2.5 The data set of load energization case study $\ldots \ldots \ldots \ldots \ldots \ldots \ldots \ldots \ldots \ldots \ldots \ldots$

2.6 Input data form for load energization case study $(\mathrm{R}$ in , $\mathrm{L}$ in $\mathrm{mH}$, and $\mathrm{C}$ in $) \ldots \ldots 33$

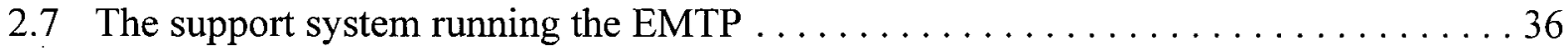

2.8 Characteristic parameters and case study comparison for step size of $50 \mathrm{~s}$ (solid) and 100

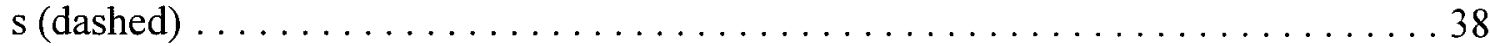

2.9 The maximum voltage amplitude of the first peak $\ldots \ldots \ldots \ldots \ldots \ldots$

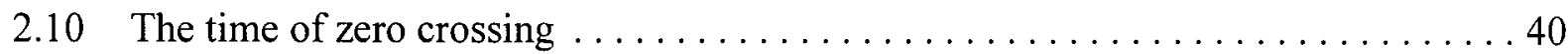

2.11 Output voltage waveform comparison $\ldots \ldots \ldots \ldots \ldots \ldots \ldots \ldots \ldots \ldots \ldots \ldots$

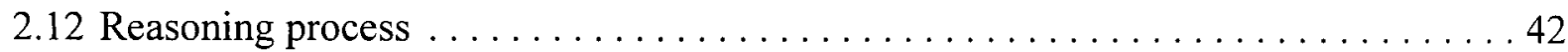

3.1 Evaluation of overvoltage factors dependent on type of operation and system [1] . . 52

3.2 Network configuration for switching surge case study $\ldots \ldots \ldots \ldots \ldots \ldots \ldots \ldots$

3.3 Comparison between field test (solid) and constant parameter line model (dashed) in

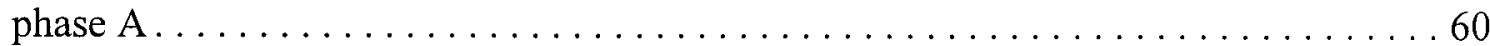

3.4 Comparison between field test (solid) and constant parameter line model (dashed) in

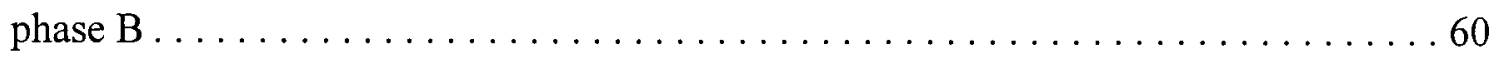


3.5 Comparison between field test (solid) and constant parameter line model (dashed) in phase $\mathrm{C}$....

3.6 Tower configuration of Jaguara-Taquaril line. All measurements in meters, earth resistivity $=100 \mathrm{~m}$

3.7 Comparison between field test (solid) and frequency dependent line (dashed) in phase A

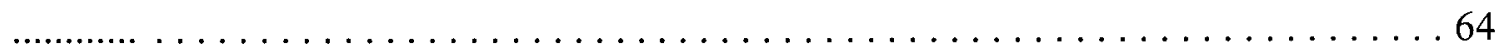

3.8 Comparison between field test (solid) and frequency dependent line (dashed) in phase B .64

3.9 Comparison between field test (solid) and frequency dependent line (dashed) in phase C

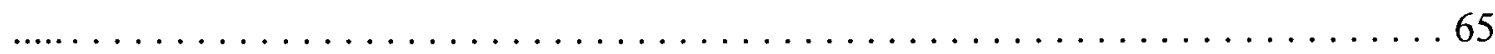

3.10 Comparison between frequency dependent line (solid) and constant parameter line

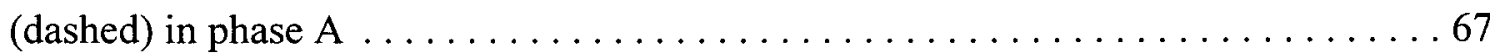

3.11 Comparison between frequency dependent line (solid) and constant parameter line (dashed) in phase B

3.12 Comparison between frequency dependent line (solid) and constant parameter line

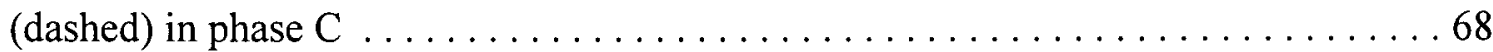

3.13 Zero sequence current for Jaguara case study $\ldots \ldots \ldots \ldots \ldots \ldots \ldots \ldots \ldots \ldots \ldots$

3.14 Three phase currents for Jaguara case study . . . . . . . . . . . . . . . . . 69

3.15 Comparison between frequency dependent line (solid) and constant line (dashed) for

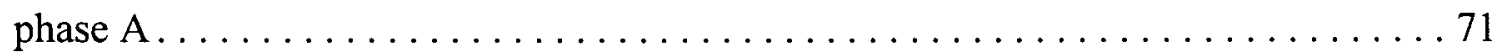

3.16 Comparison between frequency dependent line (solid) and constant line (dashed) for

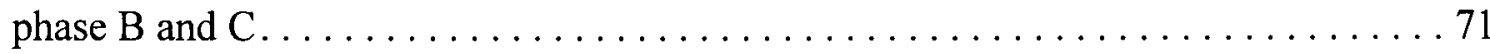

3.17 Zero sequence current and current in phase $\mathrm{A} \ldots \ldots \ldots \ldots \ldots \ldots \ldots \ldots \ldots \ldots \ldots \ldots$ 
3.18 Positive sequence inductance of the three-phase line ................. 72

3.19 Positive sequence resistance of the three-phase line $\ldots \ldots \ldots \ldots \ldots \ldots \ldots \ldots$

3.20 Zero sequence inductance of the three-phase line $\ldots \ldots \ldots \ldots \ldots \ldots \ldots \ldots \ldots$

3.21 Zero sequence resistance of the tree-phase line $\ldots \ldots \ldots \ldots \ldots \ldots \ldots \ldots \ldots$

3.22 Overvoltages with shunt reactor (solid) and without shunt reactor (dashed) in phase A . .77

3.23 Overvoltages with shunt reactor (solid) and without shunt reactor (dashed) in phase B . 78

3.24 Overvoltages with shunt reactor (solid) and without shunt reactor (dashed) in phase C . .78

3.25 Effect of trapped charges on switching surge (1.0 p.u. on phase A) $\ldots \ldots \ldots \ldots 80$

3.26 Effect of trapped charges on switching surge $(-1.0$ p.u. on phase $B) \ldots \ldots \ldots \ldots 80$

3.27 Effect of trapped charges on switching surge $(0.5$ p.u. on phase $C) \ldots \ldots \ldots \ldots 81$

3.28 Overvoltages with closing resistors (solid) and without closing resistors (dashed) at phase

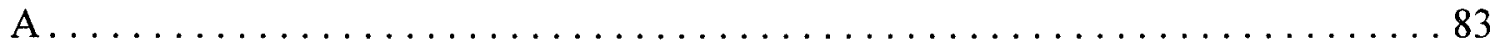

3.29 Overvoltages with closing resistors (solid) and without closing resistors (dashed) at phase B . . . . . . . . . . . . . . . . . . . . . . . . . . . . 84

3.30 Overvoltages with closing resistors (solid) and without closing resistors (dashed) at phase C . . . . . . . . . . . . . . . . . . . . . . . . . . . . . . . . . . . . . . 84

3.31 The effect of line length on the overvoltages in phase A: original line (solid), shorter line (dashed) 86

3.32 The effect of line length on the overvoltages in phase B: original line (solid), shorter line (dashed) 
3.33 The effect of line length on the overvoltages in phase C: original line (solid), shorter line (dashed)

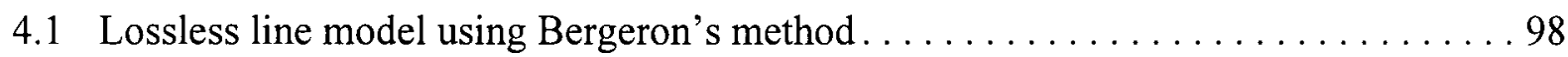

4.2 Lossless transmission line model . . . . . . . . . . . . . . . . . . . 100

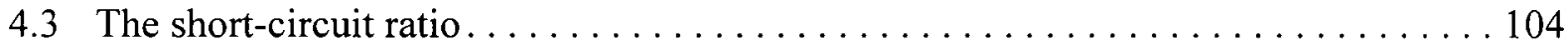

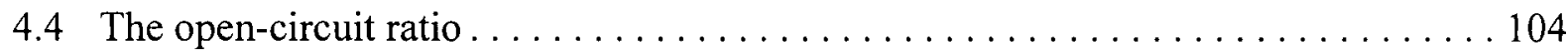

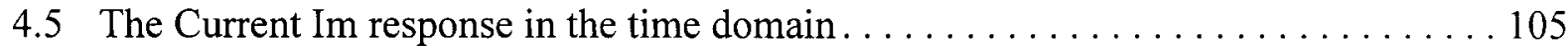

4.6 The current Ik response in the time domain ..................... 105

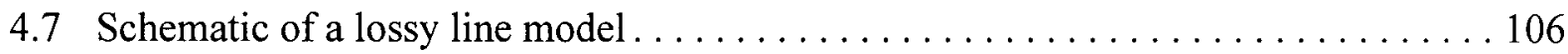

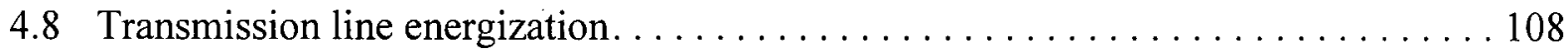

4.9 The constant parameter model response. . . . . . . . . . . . . . . . . . . . . 109

4.10 The new line model response .............................. 109

4.11 The $\pi$-circuit line model response .......................... 110

4.12 The power electronics case................................ 113

4.13 The field test recording for the drive system $\ldots \ldots \ldots \ldots \ldots \ldots \ldots \ldots \ldots \ldots \ldots$

4.14 Simulation results for the new line model $\ldots \ldots \ldots \ldots \ldots \ldots \ldots \ldots \ldots \ldots \ldots \ldots$

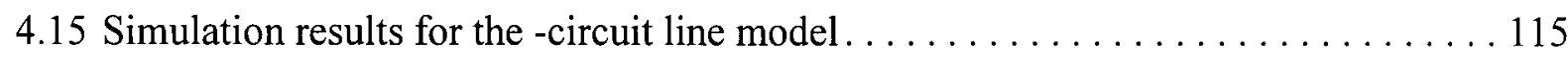

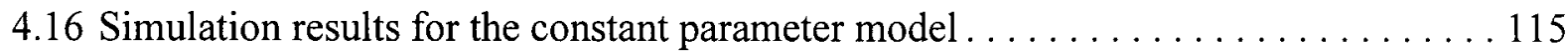

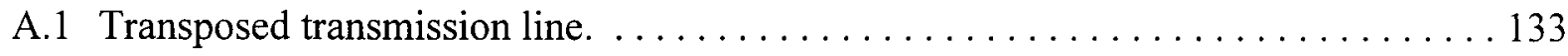

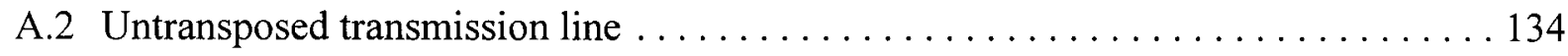

A.3 Reclosing of overhead lines with trapped charges $\ldots \ldots \ldots \ldots \ldots \ldots \ldots \ldots \ldots$ 


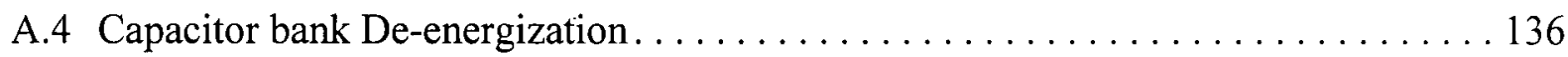

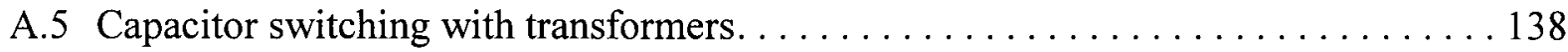

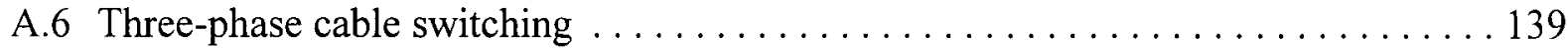

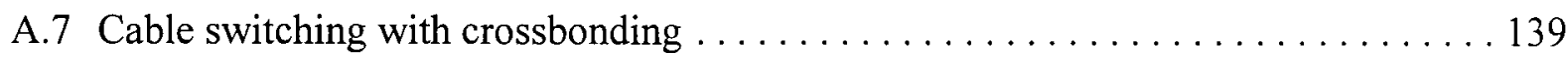

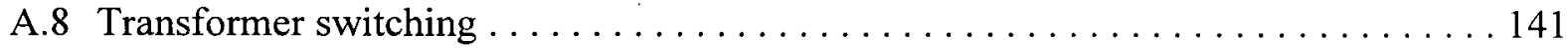

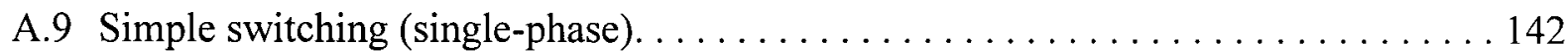

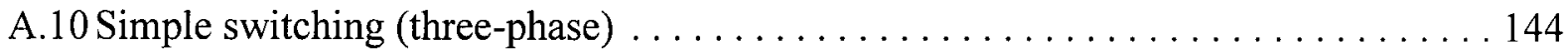

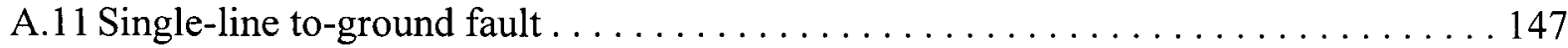

A.12 Fault analysis with frequency dependence $\ldots \ldots \ldots \ldots \ldots \ldots \ldots \ldots \ldots \ldots \ldots \ldots \ldots \ldots$

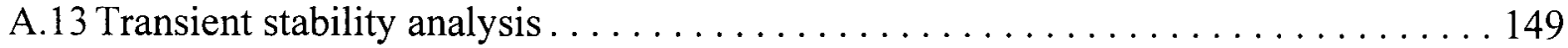

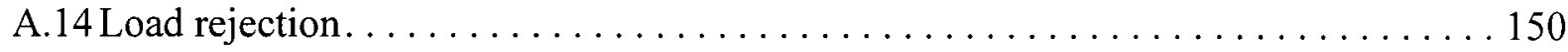

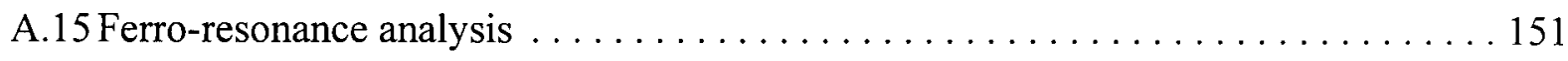




\section{List of Tables}

2.1 Summary of EMTP models for transmission line and applications $[54] \ldots \ldots \ldots 12$

2.2 Conventional analysis programs and knowledge-based expert systems $[32] \ldots \ldots 14$

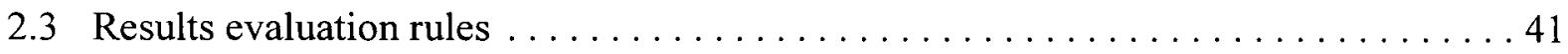

3.1 Network parameters influencing the switching overvoltages $[1] \ldots \ldots \ldots \ldots \ldots$

3.2 Network steady-state nodal voltages with shunt compensation $\ldots \ldots \ldots \ldots$

3.3 Network steady-state nodal voltages without shunt compensation . . . . . . . 76

3.4 Network steady-state nodal voltages with shunt compensation doubled $\ldots \ldots \ldots 77$

3.5 Network steady-state nodal voltages with $100 \%$ shunt compensation $\ldots \ldots \ldots 77$

3.6 Some derived rules from the knowledge base for switching surge transients . . . . 90

D.1 Data input file for the switching surge case study $\ldots \ldots \ldots \ldots \ldots \ldots \ldots$

D.2 Output file for switching surge case study $\ldots \ldots \ldots \ldots \ldots \ldots \ldots \ldots \ldots$

D.3 The fd Data input file for Jaguara case study $\ldots \ldots \ldots \ldots \ldots \ldots \ldots \ldots \ldots$

D.4 Input data file for Jaguara case study using frequency dependent line model $\ldots \ldots 176$

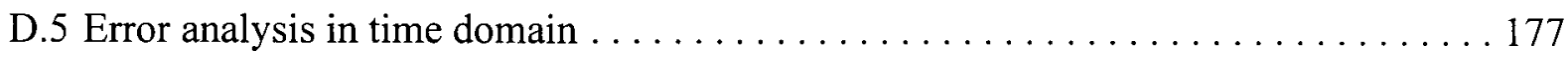




\section{Acknowledgment}

I would like to express my deepest gratitude to my thesis supervisors, Dr. Hermann Dommel and Dr. Takahide Niimura, whom I respect and admire tremendously, for their constant guidance and help throughout this work. Both their background and expertise in the areas of electromagnetic transients simulation and artificial intelligence not only made this work possible but also enriched my personal and professional life. Thank you both very much for your encouragement and support.

I further appreciate the cooperation and joint work with dear colleagues and friends, Daniel Lindenmeyer, whose suggestions for the improvement of the results evaluation process had been gladly accepted; Sebastian Henschel, whom I thank for his patience and encouragement during the development of the new line model; Antonio Carlos Siqueira de Lima, for providing the power electronics case study and field test results.

The financial assistance of the Natural Sciences and Engineering Research Council of Canada, and of B.C. Hydro \& Power Authority, through funding provided for the NSERC-B.C. Hydro Industrial Chair in Advanced Techniques for Electric Power Systems Analysis, Simulation and Control is gratefully acknowledged. The financial support of the Ministry of Education and Scientific Research of Libya, and the Canadian Bureau of International Education (CBIE) is also appreciated.

A warm and special thanks to all my friends and colleagues in the Power Group; their friendship through the past years have been a constant source of knowledge, enjoyment and energy. Thank you all and the best luck in your research. Thanks to all the Professors and staff in the Department 
of Electrical and Computer Engineering who taught, guided and helped me constantly.

Finally, I would like to thank my wife Samira, my brother Ahmed, my lovely daughters Omama, Khadija and Safia, and my parents for their never-ending patience, encouragement and sensitive understanding. I am grateful for their love and support through the years. 


\section{Chapter 1}

\section{Introduction}

\subsection{Overview}

The design of electric power systems requires a precise knowledge of the magnitude and duration of overvoltages that might occur and faults. Overvoltages appear due to lightning discharges, switching operations, and faults [55]. The insulation of transmission lines and terminal equipment, such as transformers and circuit breakers, must either be able to withstand the stresses imposed by these transient overvoltages, or surge arresters must be able to

protect the equipment from them. To co-ordinate the insulation levels of equipment with the protective level of surge arresters is referred as "insulation coordination".

The most practical and effective way currently to study the different types of transient overvoltages, their effects and the design of adequate insulation levels is through simulations with 
computer programs. The most widely known of these programs is the Electromagnetic Transients Program (EMTP) [5]. The EMTP is a combination of mathematical models and solution techniques representing the different components of the electrical network and their interpolations. The EMTP represents each component through equivalent resistances and history current sources obtained from mathematical models. Finally, the whole electrical systems is solved using numerical methods to solve the resulting simultaneous equations.

The EMTP is well tested, powerful, and flexible. However, one drawback of the EMTP is its unfriendly user interface, which limits the effectiveness and usability of the program. There are also multiple choices of models of network elements now offered in the EMTP, and users must choose the proper models that best suit the specific study. For example, a transmission line would be represented accurately enough by a $\pi$-circuit for sub-synchronous resonance studies, while a transient recovery voltage study requires a distributed parameter model.

Many efforts have been made to improve the user interface of the EMTP during the last few years. An Integrated Engineering Simulation Environment (IESE) [22][23][24] was proposed to solve the user interface problem with the EMTP, in which a graphic oriented interface is integrated with a modular engineering database.

A data modularization concept, which was incorporated into the alternative transient program (ATP) version of the EMTP [25], has been used in [26] to simplify the data input. A supporting subroutine called "Data Base Module" allows the user to create component modules that will be used later as library modules. 
A new approach was presented in [27] and [75] to use MATLAB [28] for the development of a new EMTP. The benefits of the MATLAB environment, such as toolboxes and the graphic user interface (GUI), will then be available to the EMTP. However, the execution time of MATLAB programs is much longer than that of the original EMTP, because MATLAB uses an interpreter rather than a compiler at the present time.

ATPDRAW [29] was developed as a mouse-driven preprocessor for the ATP version, where the user can build up a circuit by choosing components from menus. For the DCG/EPRI version of the EMTP, a graphical user interface is being developed by Ontario Hydro, Canada. The Manitoba HVDC Research Center has developed a graphical user interface for the EMTDC [76]. The Power Research Group at the University of British Columbia also has developed a graphical user interface for the UBC version of the EMTP (MicroTran) [16] and for the Real Time Power System Simulator (OVNI) [77].

Although the ease of data entry may be solved by the above mentioned techniques, such as graphic displays and data modularization, the difficulty of proper modeling of power systems remains basically unchanged. Also, data validation and data sanity checks have not yet been fully implemented in the EMTP.

The problem of selecting the proper models of power system elements is not as easy as it may appear. Each model has its unique features such as frequency range, time step, maximum time, and the type of phenomena being studied. The selection of the proper model is usually made based on engineering experience and judgment, which often is not available to many of 
the users. For example, the selection of the $\pi$-circuit line model depends on length $(l)$ of the line as well as on the frequency range of the transient phenomena $(f)$ as shown in the following equations [79]:

$$
\begin{gathered}
l<\frac{10,000}{f} \mathrm{~km} \text { for overhead lines, } \\
l<\frac{3,000}{f} \mathrm{~km} \text { for underground cables }
\end{gathered}
$$

(For $f=60 \mathrm{~Hz}, l<167 \mathrm{~km}$ for overhead lines, $l<50 \mathrm{~km}$ for cables). These approximate rules are derived from frequency domain comparisons of the open and short-circuit behavior of the $\pi$-circuit (nominal $\pi$ ) and the exact solution (exact equivalent $\pi$ ).

Furthermore, the issue of data validation requires all of the analyst's knowledge about the reasonableness of the data and the frequency range over which the model is valid. Also, the results evaluation of the EMTP is not simple, because the answers are different for different types of studies. Results evaluation depends on the model used, the extent of the system represented, and other factors.

To use the EMTP properly, it is best to have an experienced user to oversee the initial stages of the EMTP learning curve. However, a beginning user does not always have a supervisor who is familiar with the procedures of EMTP simulation and the phenomena to be simulated. There are various EMTP applications to power system transient studies in the literature, but the expertise is normally personalized and not stored as electronically accessible information. 
All the factors of power system transients simulations using the EMTP imply that an intelligent support system approach could be effective in dealing with the above mentioned problems. The main advantage of this approach is its ability to deal with knowledge in an easily accessible form. This was the motivation for adopting the intelligent support systems approach to power system transients simulations.

This thesis presents an intelligent system to support simulations with EMTP and similar programs. The work is composed of three parts: the first part presents the development of a prototype intelligent support system, the second part introduces the knowledge base for switching surge studies, and the third part describes the development of a new line model for the representation of short overhead transmission lines and underground cables.

The intelligent support system works in three stages. First, the support system selects a base case from a case database, to be modified to meet the user's requirement. Second, the expert system checks the syntax and validity of the new case data. Finally, the EMTP solution is checked to see whether the chosen models are appropriate for the frequencies contained in the solution, for the degree of imbalance, and for other criteria. If they are not appropriate, better models will be recommended to the user.

For the knowledge base, switching surge transients have been chosen as an example. The knowledge base will solve the problem of selecting the proper model for representing power system components in the EMTP according to problem specifications. It will help in checking the validity of the data used to represent the simulated transient phenomena, and will give 
some suggestions to the user to correct his case data before the simulation. It will also help in the evaluation of the results of the EMTP simulation, using the knowledge of the phenomena being simulated. From this knowledge base, the idea of developing a new line model for short lines and cables evolved as one of the practical rules that is recommended for the proposed intelligent support system.

The new EMTP transmission line model for the representation of short overhead lines and underground cables will overcome the limitation of having to use a time step size not larger than the shortest travel time. The error analysis for the short-circuit and open-circuit responses shows that the new line model has a filtering effect for higher frequencies. In a comparison with actual field test measurements, it could be shown that the new line model is suitable for a reasonable representation of short overhead transmission lines and underground cables.

\subsection{Thesis Objectives}

1. Developing an intelligent support system to support practicing engineers and engineering students who are not familiar with EMTP simulations [52][70][71][74]. This expert system will be composed of three parts. First, the expert system selects a base case data from a case database, to be modified later to meet the user's own goal. After that, the expert system checks the validity of the modified case data. Finally, the EMTP solution is verified for the user's specific data case.

2. Developing a knowledge base, with switching surge overvoltages used as an example. From this knowledge base, we can derive some practical rules for switching surge tran- 
sients. These practical rules will be used in the proposed EMTP intelligent system. The objective of this part of the thesis is to give the simple and approximate rules for the support system. These rules will provide the beginning EMTP users and practicing engineers with a simple scheme based on the experience of EMTP uses.

3. Developing a new EMTP line model for the representation of short overhead transmission lines and cables [64][72][73]. It overcomes the limitation of having to use a time step size not larger than the shortest travel time. This modelling approach is proposed by one of the practical rules for the intelligent support system.

\subsection{Thesis Outline}

Due to the diversity of the topics handled in this thesis (e.g. expert systems, switching overvoltages, transmission line models, etc.) it was decided not to include one literature review chapter of all the topics by itself. Rather, literature reviews will be included with each chapter for the relevant topics.

Chapter Two describes the development of an intelligent support system to help engineers and students in the proper use of the EMTP.

Chapter Three presents the development of a knowledge base for switching surge overvoltages. A typical study for switching transient overvoltages is also introduced.

Chapter Four presents a new EMTP line model for the representation of short transmission lines and cables. 
Chapter Five gives the conclusions derived from this work, and suggests recommendations for future work in this area. 


\section{Chapter 2}

\section{Intelligent Support System}

\subsection{Introduction}

The electromagnetic transient program (EMTP) [5] is a general-purpose computer program for simulating fast transient effects in electric power systems. The EMTP is a widely used program for transient analysis, in industry as well as in universities. There are several versions available. The program features an extremely wide variety of modeling capabilities, encompassing electromagnetic and electromechanical transients ranging in duration from microseconds to seconds.

The EMTP is reasonably well tested, powerful, and flexible for various types of transient studies. However, the main drawback of the EMTP is the difficulty of building a proper representation of the power system to be studied. A high level of expertise is required for the 
analysis of transient phenomena in power systems. The users are assumed to have a basic knowledge of the phenomena to be simulated, and at the same time they are required to have a certain familiarity with the input data format of the EMTP. In this situation, it is best to have an experienced EMTP user to overlook the initial stages of the EMTP learning curve. However, it is not always possible for the beginning user to have a supervisor who is familiar with both the procedures of EMTP simulation and the phenomena to be simulated.

Many efforts have been made to improve the use of the EMTP during the past few years. Although the ease of using the program may be improved by different techniques, such as graphic display [22] [29] and data modularization [26], the difficulty of proper modeling of the power system being studied remains basically unchanged. Also, possible user support in data validation and results evaluation has not yet been fully investigated in the EMTP simulation environment.

This chapter describes the development of an intelligent support system to help engineers and students in the proper use of the EMTP [52][70][71][74] and similar programs, such as EMTDC and ATP. This chapter is composed of eight sections. The next section introduces an overview of expert systems and their applications in power systems. Section 3 explains the need for expert system approaches. Section 4 introduces the structure of the proposed intelligent support system. Section 5 presents the implementation of a prototype support system. Section 6 describes a simple energization case study as a base case example. Section 7 introduces case study results evaluation, and section 8 summarizes the conclusions and 
comments about the proposed intelligent system.

\subsection{The Need for Using an Expert System Approach}

Unfortunately, the problem of selecting the proper models of power system is not as simple as it may appear. Each model has its unique features such as frequency range, time step, maximum time, and the type of phenomena being studied. For example, hysteresis effects may be important in ferresonance but unimportant in inrush current studies. The selection of the proper model is usually made based on engineering experience and judgment, which often is not available to many of the users. As an example, Table 2.1 shows the different models available for transmission lines, and the recommended applications for each model.

Furthermore, the issue of data validation requires all of the analyst's knowledge about the reasonableness of the data and the frequency range over which the model is valid. For example, the $\pi$-circuit type of transmission line model is only valid for lower frequencies from 0 to $200 \mathrm{~Hz}$ for a line length of $50 \mathrm{~km}$. There are many other model limitations as well, e.g., voltages should not be higher than 1.2 p.u. if transformer saturation is ignored. Also, the results evaluation of the EMTP is not simple, because answers are different for different types of studies. Results evaluation depends on the model used, the extent of the system represented, and other factors.

All the characteristics of power system transients simulations using the EMTP suggest that an intelligent support system approach could be capable in dealing with the above designated issues. The most significant benefit of an intelligent support system approach is its capability 
to deal with knowledge in an easily accessible form. This will make the experience and analysis of power system transients simulations using the EMTP available for all users.

Table 2.1: Summary of EMTP models for transmission line and applications [54]

\begin{tabular}{|c|c|}
\hline Model & Best Fit For \\
\hline A) Frequency Independent Line & $\begin{array}{l}\text { Best for parametric studies, power frequency or low frequency } \\
\text { phenomena, or where exact data is unavailable }\end{array}$ \\
\hline $\begin{array}{l}\text { Positive sequence lumped parameter } \\
\text { R-L representation, and positive and } \\
\text { zero sequence lumped parameter } R-L \\
\text { representation }\end{array}$ & $\begin{array}{l}\text { Unswitched line, unbalanced conditions, load flow, initial } \\
\text { conditions for balanced systems, and remote source equivalent. }\end{array}$ \\
\hline$\pi$-section representation & $\begin{array}{l}\text { - Can be used for switched lines, although not recommended. } \\
\text { - Data similar to Transient Network Analyzer (TNA) data. }\end{array}$ \\
\hline $\begin{array}{l}\text { Distributed parameter transposed line } \\
\text { model }\end{array}$ & $\begin{array}{l}\text { General purpose studies, switched lines, lightning and high } \\
\text { frequency studies, and where typical and line-specific data is } \\
\text { available. }\end{array}$ \\
\hline $\begin{array}{l}\text { Distributed parameter untransposed } \\
\text { line model (K.C. Lee's model) }\end{array}$ & $\begin{array}{l}\text { Untransposed lines, high grounding resistivity and unbalanced } \\
\text { circuits, general purpose studies, modal analysis, and traveling } \\
\text { wave analysis. }\end{array}$ \\
\hline B) Frequency Dependent Line & $\begin{array}{l}\text { When frequency dependence is important, for switching surge } \\
\text { studies, and those studies dealing with transient system } \\
\text { resonances in excess of } 1 \mathrm{kHz} \text {. }\end{array}$ \\
\hline J. Marti model & $\begin{array}{l}\text { Can be used for transposed or untransposed lines (assumed } \\
\text { constant transformation matrix) }\end{array}$ \\
\hline L. Marti model & $\begin{array}{l}\text { Can be used for untransposed lines and cables (transformation } \\
\text { matrix frequency dependent) }\end{array}$ \\
\hline Corona Model & $\begin{array}{l}\text { Can be used for transposed or untransposed lines with corona } \\
\text { effects (assumed constant transformation matrix) }\end{array}$ \\
\hline
\end{tabular}




\subsection{Expert Systems-Background}

Expert systems are a sub-field of artificial intelligence (AI) methods. Artificial intelligence is the branch of computer science that studies how computers can be used to simulate and duplicate functions of the human brain.

The name "expert system" is used interchangeably with the term "knowledge-based system". An expert system is a computer program which embodies human knowledge and understanding in a way which imitates a human expert who can solve specific types of problems. Such programs can be used by non-experts to improve their problem solving abilities or by experts to provide consistent and perhaps, faster approaches to the application of knowledge [30].

Expert systems have been successfully applied to a variety of areas. These include diagnosis, planning, intelligent tutoring, monitoring, control, and scheduling [31]. Also, the expert system has the ability to deal with knowledge in a symbolic form. This makes it easier to incorporate knowledge which is only present as human expertise in heuristic form.

Moreover, the expert system approach can handle real world data which involves uncertain, missing, and inaccurate data.

The expert system approach is more effective than conventional analysis techniques in dealing with problems where judgment and experience are needed. The differences between knowledge-based expert systems and conventional analysis programs outlined in [32] are summarized in Table 2.2 . 
Table 2.2:Conventional analysis programs and knowledge-based expert systems [32]

\begin{tabular}{||l|l|}
\hline \multicolumn{1}{|c|}{ Conventional Analysis Programs } & Knowledge-Based Expert Systems \\
\hline $\begin{array}{l}\text { Stated equations which can be proven. If } \\
\text { correct numerical data is provided, a correct } \\
\text { answer will result. }\end{array}$ & $\begin{array}{l}\text { Usually based on rules of thumb that are } \\
\text { generally reliable and cannot be reduced to } \\
\text { formulae or numbers. }\end{array}$ \\
\hline Provides answers only & Can explain its logic and reasoning \\
\hline Needs all data called for to operate & Can function with incomplete data \\
\hline $\begin{array}{l}\text { Often programmed in isolation from domain } \\
\text { experts and users }\end{array}$ & $\begin{array}{l}\text { Development team includes domain experts } \\
\text { and users }\end{array}$ \\
\hline $\begin{array}{l}\text { May be extremely difficult to examine } \\
\text { imbedded knowledge }\end{array}$ & $\begin{array}{l}\text { Provides capability to easily examine } \\
\text { knowledge base }\end{array}$ \\
\hline
\end{tabular}

\subsubsection{Expert System Components}

The expert system has three components, as shown in Figure 2.1 [33]. These components are as follows:

\subsubsection{Knowledge Base}

A knowledge base is a collection of data, rules, inference, and procedures organized into frames, scripts, rules and other formats. It contains everything necessary to understand, formulate and solve the problem [33].

Also, it includes two basic elements: facts such as problem situations, and special heuristics or If-Then rules that direct the use of knowledge to solve the problem. The heuristics express the 
informal judgment knowledge of the application area. The information in the knowledge base is incorporated into a computer program by a process called knowledge representation.

Generally, the knowledge base is written in a natural language for ease of development by inexperienced computer programmers [33].

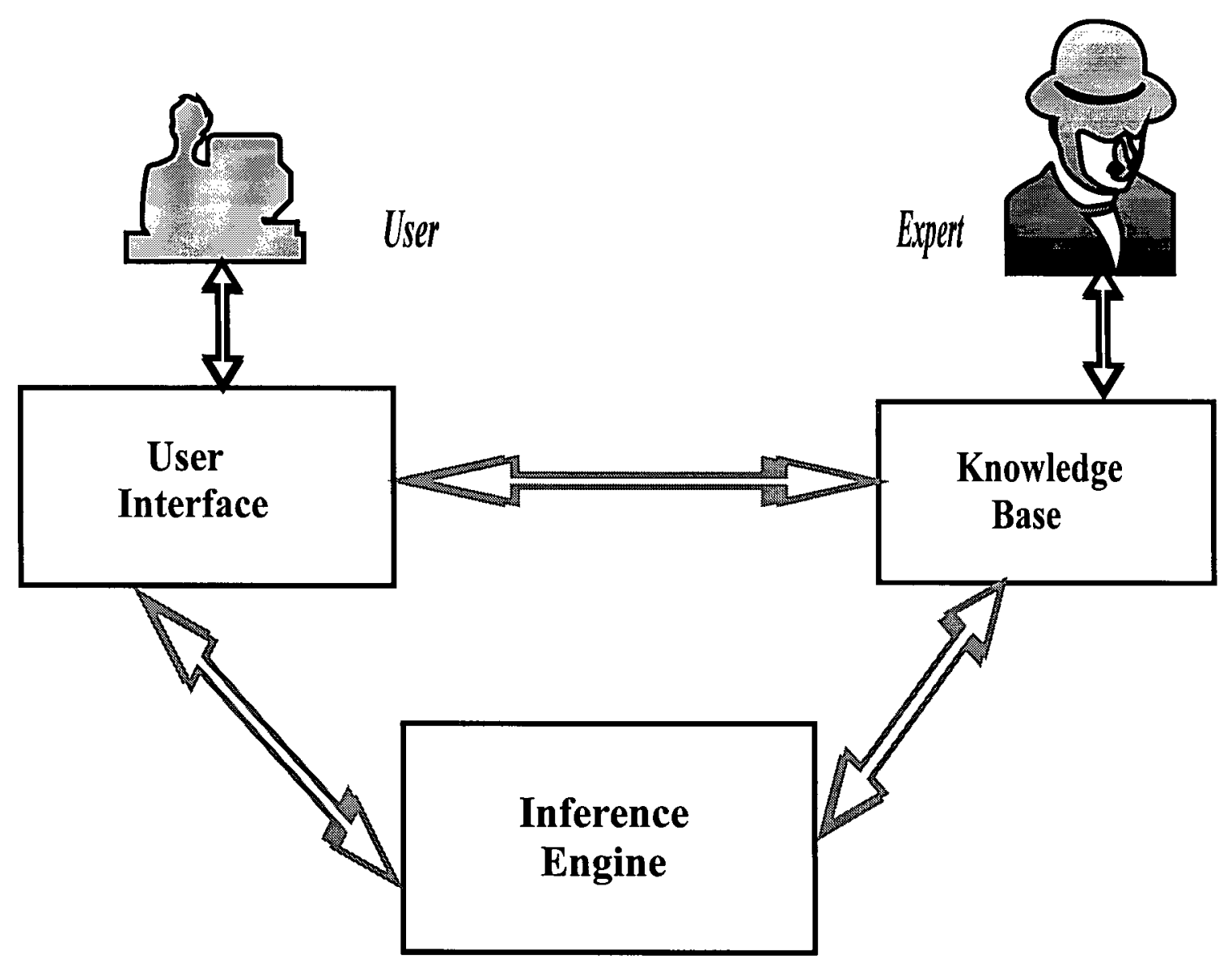

Figure 2.1 Expert system components 


\subsubsection{Inference Engine}

The inference engine is the brain or the thinking part of the expert system. It is a computer program which processes the knowledge contained in the knowledge base. It contains methods to reason with information in the knowledge base and to arrive at conclusions. The inference engine responds to user input and to changes that occur in the knowledge base [33].

\subsubsection{User Interface}

The user interface is the facility which accommodates communication between the user and the computer. In some cases this communication may be by line command or through menus. It is through this facility that the inexperienced user can be trained in the use of the knowledge contained in the knowledge base [33].

\subsubsection{Applications of Expert Systems to Power Systems}

Interest in the application of expert systems to power systems is growing strongly. There is a need for expert systems in response to the inherent difficulties that are associated with the daily operation and future planning of very large and complex power systems.

For example, power system disturbances which may lead to system collapse are relatively fast, and the time available to decide and take corrective action is very limited. As human beings are incapable of judging and correcting situations within a very short time, based on a large amount of incomplete information, it is strongly hoped that expert systems will be able to fill the gap between human capability and power system complexity.

In recent years, many power systems applications of expert systems have been implemented 
successfully [34][35][36][37]. They can be classified as follows:

Monitoring: The monitoring category heads the development of expert systems in power system. Fault diagnosis and alarm processing are the dominant applications in the monitoring category. The fault diagnosis area includes fault detection, location of the faulty section, and prediction of the behavior of the system in the emergency state. Alarm processing usually includes the real time monitoring of sequential events and the detection of malfunctioning of protective devices.

Control: The control area applications include the normal, emergency, and restorative states. Control of the normal state depends heavily on state estimation, load forecasting, system stability and voltage and VAR control. The control of emergency state includes corrective actions against voltage collapse and countermeasures for maintaining system security. The restorative state deals with system restoration of transmission lines and switching operations in substations.

Planning: Operational planning includes system structure planning, generation scheduling, reactive power scheduling, system stabilization, and security assessment.

Education \& Training: According to a recent survey [38], many applications of expert systems are used for training simulators for various tasks. These training simulators are used by operators and students.

System Analysis: System analysis applications include system model validation, load flow 
analysis, and voltage instability studies.

\subsubsection{Integration of Expert Systems with Power Simulation Programs}

An expert system can be integrated with conventional power simulation programs, to combine the ability and strength of both environments. The conventional power simulation program can efficiently solve the defined problem, while the expert system is much better at handling heuristic knowledge. The integration of the expert system with power simulation programs will overcome the difficulty of incomplete simulation knowledge.

This approach will be used in the proposed research project, to integrate a prototype expert system with the EMTP. This approach is new and only a few papers can be found on it [39][40]. These two papers present a prototype expert system which was incorporated with a power converter simulation program to reduce the design time and costs. The knowledgebased system imitates an experienced designer's approach in choosing the proper topology and component values to satisfy the design specifications. The converter responses are calculated with the converter simulation program to verify the design, using the knowledgebased system. If there is any violation in design specifications, the knowledge-based system will modify the design parameters and continue the design procedure until all specifications are met. The knowledge-based system also prepares the SPICE-like input file for the converter simulation program. 


\subsubsection{Tools and Shells of Expert Systems}

Most of the knowledge-based systems are created using expert system shells, because they are easy to use when compared with programming languages. Each shell is suitable for a specific domain. A comprehensive list of different shells and languages used in power systems applications is presented in [34][41].

Special artificial intelligence (AI) languages such as LISP and PROLOG were frequently used [42]. These AI languages are different from conventional programming languages as they provide effective possibilities to handle symbolic information and data structures that change dynamically during run time. However, these AI language tools are limited to their machines and high-end workstations. Also, they often have difficulties in interfaces with other software systems.

Conventional programming languages such as C, FORTRAN, and Pascal can also be used to develop expert systems. These conventional programming language shells are faster, more flexible, easy to interface with other software systems and widely available as compared with traditional AI languages tools [42]. However, they require skillful programmers and a great deal of effort to work with them.

There are some popular expert system shells such as EXSYS, INSIGHT 2+, and TIMM, which are written in C, Pascal, or FORTRAN [42]. The remarkable advances in the area of developing expert system shells has provided very sophisticated shells which reduce the need to use $\mathrm{AI}$ or conventional programming languages. 


\subsubsection{A Survey of Expert System Tools}

In this section several expert system tools are reviewed [44][45][46][47][48][49][50]. The goal of this survey is to assess the functionality and performance of the expert system tools and to gain insight into their advantages and disadvantages.

\section{C Language Integrated Production System (CLIPS)}

CLIPS [44] was developed by NASA at the Lyndon B. Johnson Space Center. It was designed to overcome a number of difficulties that NASA had experienced using LISP-based tools. CLIPS is written in C to support the goals of high portability, low cost, and ease of integration with external systems.

CLIPS uses rules as its primary knowledge representation approach and its inference is based on a forward-chaining control strategy only. The source code of CLIPS is provided, to allow the developer to modify it to meet his desired application. CLIPS has some disadvantages, such as its lack of support for backward-chaining, and it does not support a graphics tool kit for building end-user interfaces.

The Fuzzy CLIPS is now available as an extended version of the CLIPS rule-base shell for representing and manipulating fuzzy facts and rules [45].

\section{Automated Reasoning Tool for Information Management (ART-IM)}

ART [46][47][48] was introduced in 1985 by Inference Corporation as a LISP-based expert system tool targeted to LISP machines. ART-IM is the company's current version 
implemented in C. It uses NASA' CLIPS as a base and has several enhancements added, including a frame system, an object oriented programming capability, and a graphic development environment called the ART-IM Studio.

\section{Level 5}

A backward-chaining expert system tool was introduced by Level Five Research in 1984. Level 5 [46][47][48] is written in Pascal and it supports backward-chaining, an English-like rule language, and certainty factors. Level 5 does not provide a frame system and its ability to emulate forward-chaining is limited to simple problems. Level 5 supports several windows, dialogue boxes, and a debug menu on Macintosh. However, it has several drawbacks, such as the absence of a graphic tool kit for building user interface applications.

\section{VAX OPS5/OPS83}

Official Production System Version 5 (OPS5) [46][47][48][49] was developed at Carnegie Mellon University. It has influenced the development of a number of expert system tools, including ART and CLIPS. It is written in Bliss (DEC's system implementation language) and executes only on DEC hardware. Also, it can be interfaced with a number of DEC-supported languages. VAX OPS5 has fast execution times and it can support the development and delivery of large expert systems. However, it is characterized by forward-chaining and it does not support backward-chaining. There is a PC version called OPS83 that is used to build up an expert system for a voltage control application [34][39]. 


\section{Knowledge Engineering System (KES)}

KES [46][47][48] was introduced by Software A\&E in 1982. The early versions of KES were implemented in LISP, but it was ported to $\mathrm{C}$ in recent versions. KES provides forwardchaining rules, backward-chaining rules, and a frame system for knowledge representation. KES also handles uncertainty using certainty factors as a measure of belief in a value.

The KES development environment for UNIX systems supports a graphical development interface based on X Windows, however it does not provide an editor. Also, KES does not support a graphic tool kit for user interface applications.

\section{COMDALE/X}

COMDALE/X [50] is an expert system shell which provides a highly effective, efficient and easy to use environment for the development and delivery of expert system applications. COMDALE/X has many built-in features and advantages that make development of an expert system easier and straightforward. These advantages include:

1. A customized windowed user interface and a hypertext facility including graphical presentations.

2. Simultaneous backward and forward chaining capabilities.

3. It includes debugging features (watch variables and rule tracing).

4. Excellent meta-knowledge capabilities, such as customized questions and explanations, 
hypertext documents, forms, and graphics.

5. Technical support is provided by Comdale Technologies (Canada) Inc. to the users of COMDALE/X. Also, the Department of Mining and Mineral Processing of UBC has offered a course in expert systems applications using COMDALE/X, and good feedback and consultations are provided.

These mentioned advantages make COMDALE/X the best choice among the surveyed expert system shells, especially for its technical support and consultation at UBC. This is why COMDALE/X is used for the current prototype support system.

\section{The principal components of a COMDALE/X expert system are:}

1. The Knowledge Base: The knowledge base can handle all types of information that humans use to make decisions in a particular domain. The knowledge base therefore contains:

2. Objects that represent factual information.

3. Classes which are the structural relationships of the facts in hierarchical classification.

4. Rules which are the complex relationships formulated between facts.

5. Procedures that are used to dictate the application of rules and the manipulation of classes and rules. 
COMDALE/X compiles a knowledge base when it is loaded for use. Maintaining and consulting a knowledge base requires loading of the system only once. Objects, classes, rules, and procedures are incrementally compiled as they are modified.

The Inference Engine: The inference engine processes the knowledge to make decisions.

The User Interface: It provides the window to observe and communicate with COMDALE/ $\mathrm{X}$ during the development and use of an expert system application.

The Utilities: They facilitate various approaches to represent and display information as well as provide security of the knowledge base.

The Hypertext Facility: It has been produced so that the system designers can easily create documents for on-line reference for use with expert system applications.

To facilitate this ease of use, the hypertext format consists of constructing a text within a text editor and saving the file as an ASCII format file.

\subsection{System Structure}

The proposed structure of the intelligent support system for EMTP simulation is shown in Figure 2.2. The support system's operation can be divided into three stages: case data selection, data validation, and results evaluation. 


\section{Case Database}

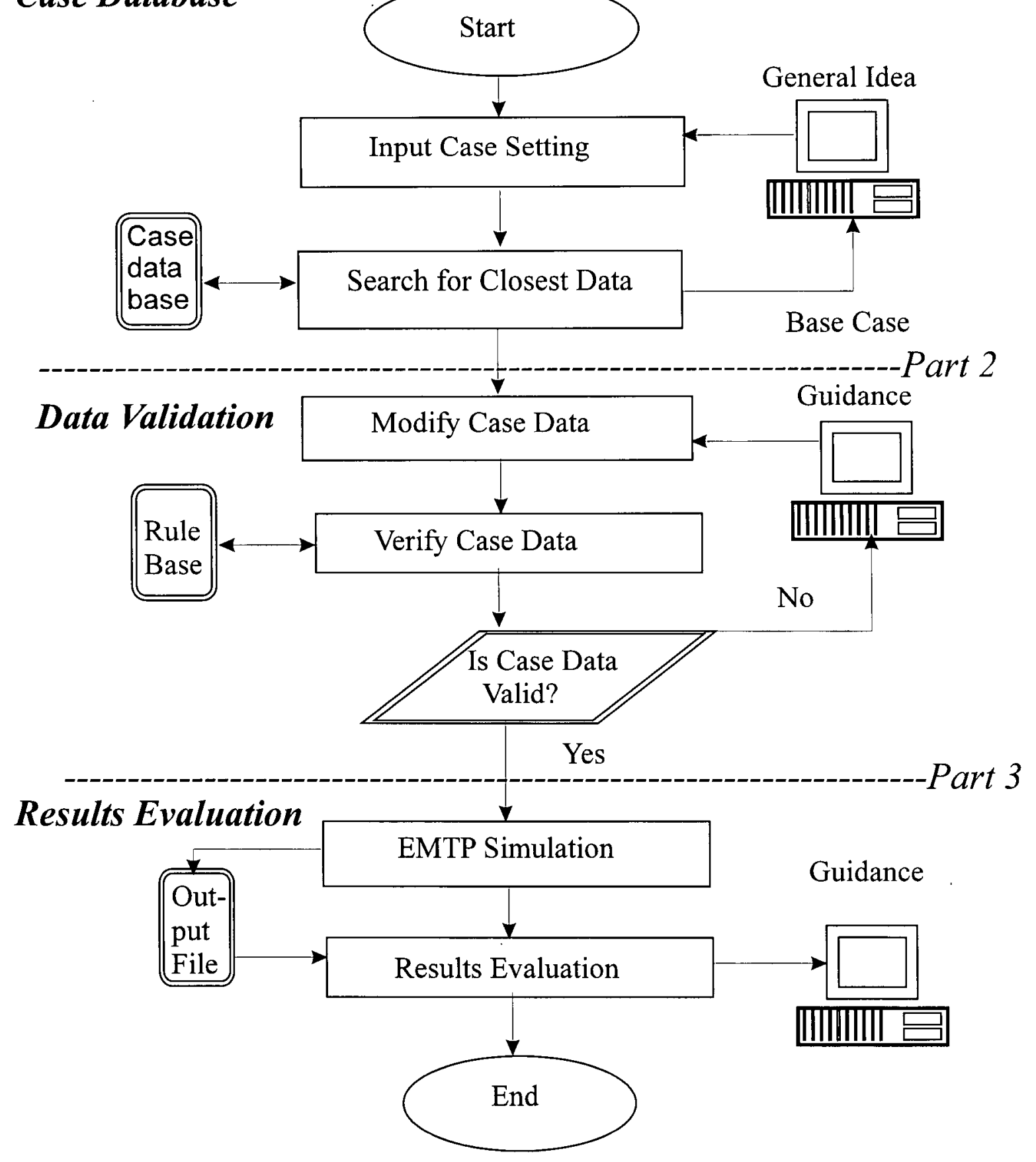

Figure 2.2 Structure of transient simulation support system 
Experienced users of EMTP have an advantage over beginners because they can choose a previously successful data set which is similar to a given simulation case. The experts can also tell what values of diverse parameters will be appropriate for the simulation. When the simulation results are obtained, they can often immediately recognize if the simulation is successful or not by the output of waveform, due mainly to their capacity of roughly estimating the expected output. This support system imitates such actions of a transient analysis expert.

\subsubsection{The Case Database}

A database that contains a collection of various power transient study cases is the most fundamental part of the proposed support system. The case-related information have been collected from EMTP experts, technical papers, and manuals, and compiled into a hypertextbased database. The case database engine locates the case data set that is closest to the system configuration and type of study of the user's base case by an interaction with the user. In many cases, inexperienced users may have a vaguely defined goal for the simulation, but through the interactive search, the system can be narrowed down to a specific case. The interactive search can also draw the context information of the target study, which will be valuable in the later stages of the support process. The hypertext data cases are classified in such a way that the user can reach a relevant data case easily through the search menus.

The selection of a particular case data by the user initiates the support system to retrieve the data set necessary for running the EMTP simulation. Since this is only a base data which 
resembles the user's case, the data is copied to a user file, in which the user can modify the data to meet the requirements of the specific case to be studied.

\subsubsection{Data Validation}

In the second stage, the support system checks the structure and the validity of the new case data that the user modified for his or her specific purposes. The support system will give the user some guidelines and explanations to correct suspicious data. Also, some hints about the system representation are provided. Such a checking procedure examines the reasonableness of the power system parameters, the frequency range over which the selected models are valid, the discrete time step, the overall length of simulation, and other factors [53][54].

From the experts' knowledge of different models of power system elements, such as transmission lines, and the phenomena being studied, certain rules have been compiled for the support system and are applied to verify the validity of the case data. If the support system finds that the modified case data is valid, it will activate EMTP to simulate this case. If not, the modification-validation procedure will be repeated until a proper case data set is obtained.

\subsubsection{Results Evaluation}

After the EMTP engine numerically solves the simulation case, the results verification step is executed, where the EMTP solution is checked whether it is reasonable. The most important tool for verifying the EMTP case results is the basic knowledge of the phenomena to be simulated. Such knowledge can be found in field test results [7][8][53][54], technical papers [1][2][3], textbooks [13][17][55][56], and EMTP experts. The support system will propose a 
list of possible corrections to the users so that they can improve their case data.

In the results evaluation process, the EMTP output waveforms are examined in the time domain. One of the potential evaluation processes is to compare the obtained results with that of the base case, because the base case is known to produce a reasonable result. If the modification is relatively minor, for example, with a slight variation of parameters, the simulation results will be reasonably similar to that of the base case. However, if the modification is extensive and there are some drastic changes in configuration, the support system no longer assumes that the results will be satisfactory. Models used in the simulation case may not be valid for the new study.

When two output curves are compared, however, it is usually not easy to simply say that they are similar or not. In real life, the truth lies rather between these statements. The application of fuzzy logic allows defining such statements mathematically by mapping them into a certain range of parameters, which can then be used to measure the degree of similarity.

\subsection{Implementation}

To test the feasibility of the proposed system, a prototype case-based expert system has been implemented to support EMTP simulation. The EMTP support system currently has the case database shown in Figure 2.3. This case database is presented in more detail in Appendix A. 
[Menu]

Power System-
Transient Studie
[Sub-Menu]

\section{Switching} Transient

Overhead Line Energization

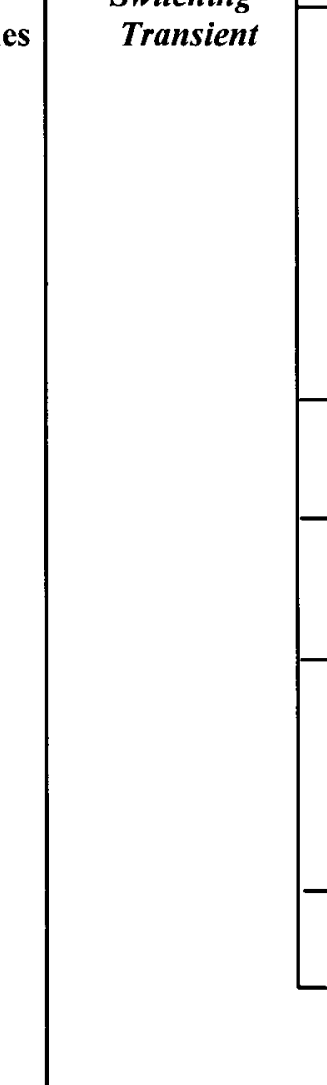

Energization with

Trapped Charges

Underground Cable Energization

\section{[Case Data]}

Overhead Line Re-

Capacitor Switching Capacitor Bank De-nergization Capacitor Bank Energization Three-Phase Cables

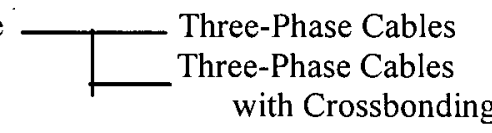

Transformer and Shunt - Transformer \& Shunt Reactor Reactor Energization Energization Load Energization Ferroresonance Analysis Interruption of Small Inductive Currents

Single Line-to-Ground Fault of an Overhead Line Single Line-to-Ground Fault of a Cable Transient Stability Analysis for Three-Phase Short Circuit Load Rejection

Resonance
Linear Resonance at Power Frequency Linear Resonance at Harmonics

Figure 2.3 The hierarchy of case database 
The MicroTran version of EMTP, developed at UBC [10], is used to run transient simulation cases [7][17][53][54]. The transient program exchanges data with the support system through an input case data file and result files. The expert system shell COMDALE/X [50] is used to build up the knowledge base of the prototype support system.

This prototype support system is installed on a PC with Pentium Pro $200 \mathrm{MHz}-\mathrm{Class} \mathrm{CPU}$ running Windows NT with $32 \mathrm{MB}$ of main memory.

This intelligent support system provides a case data library that can help users to build up their case data quickly and properly. Then, the rule-based system validates the data that was modified by the users to meet their simulation requirement.

Suspicious case data will be rejected, and the users will be prompted to correct the parameter values and models. The simulation results are examined by the waveform comparison for particular features.

In the following section a sample case study from [53] is used to show the performance of the proposed support system.

\subsection{An Energization Case Study}

\subsubsection{Case Data Selection}

A single-phase load energization transient study from [53] is used for demonstration purposes in this section. The system configuration is shown in Figure 2.4. 


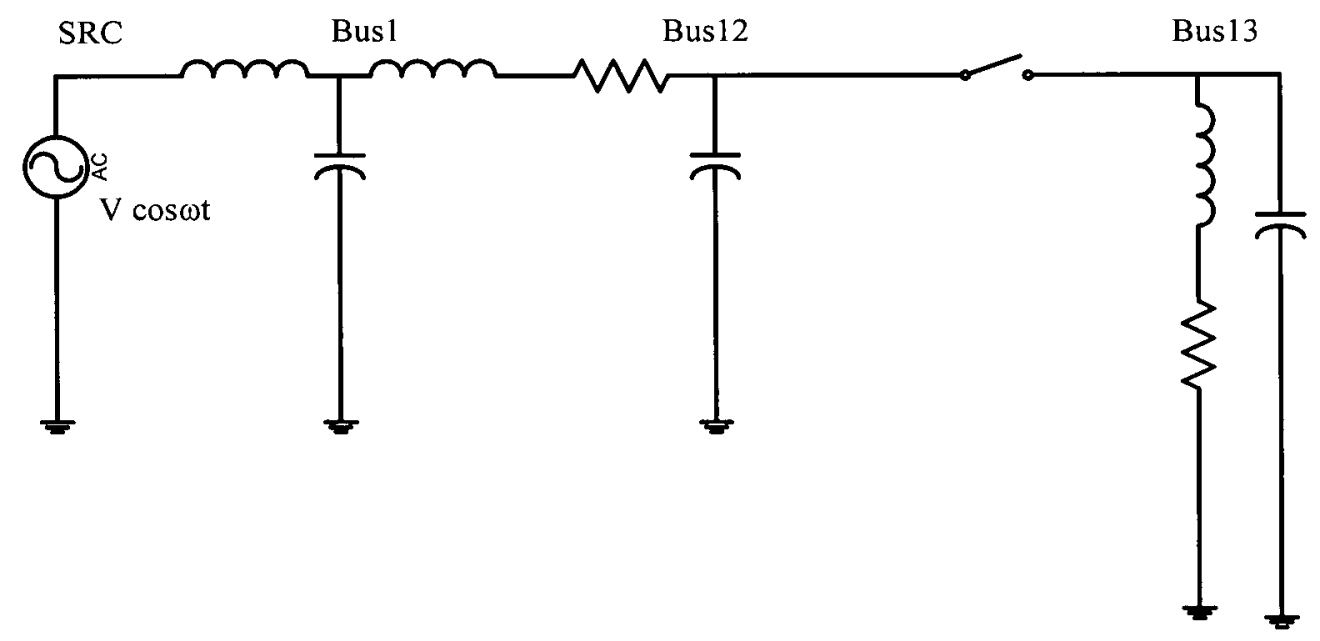

Figure 2.4 System configuration for a single-phase switching case study

Figure 2.4 represents the energization of a single-phase R-L-C load bank from a voltage source behind a Thevenin equivalent impedance through a transmission line modeled by a $\pi$ circuit.

This case data is located in the case database of the proposed intelligent support system under the switching transients category. The case data, when selected, is displayed to the user with some relevant information. The information is organized so that the user can look at the system configuration and a short description in a single frame. If the user wants more information about the case, he or she can simply jump to a detailed description by a point-and-click operation of a mouse on the hypertext data. The data file of this case study with its description is shown in Figure 2.5. 


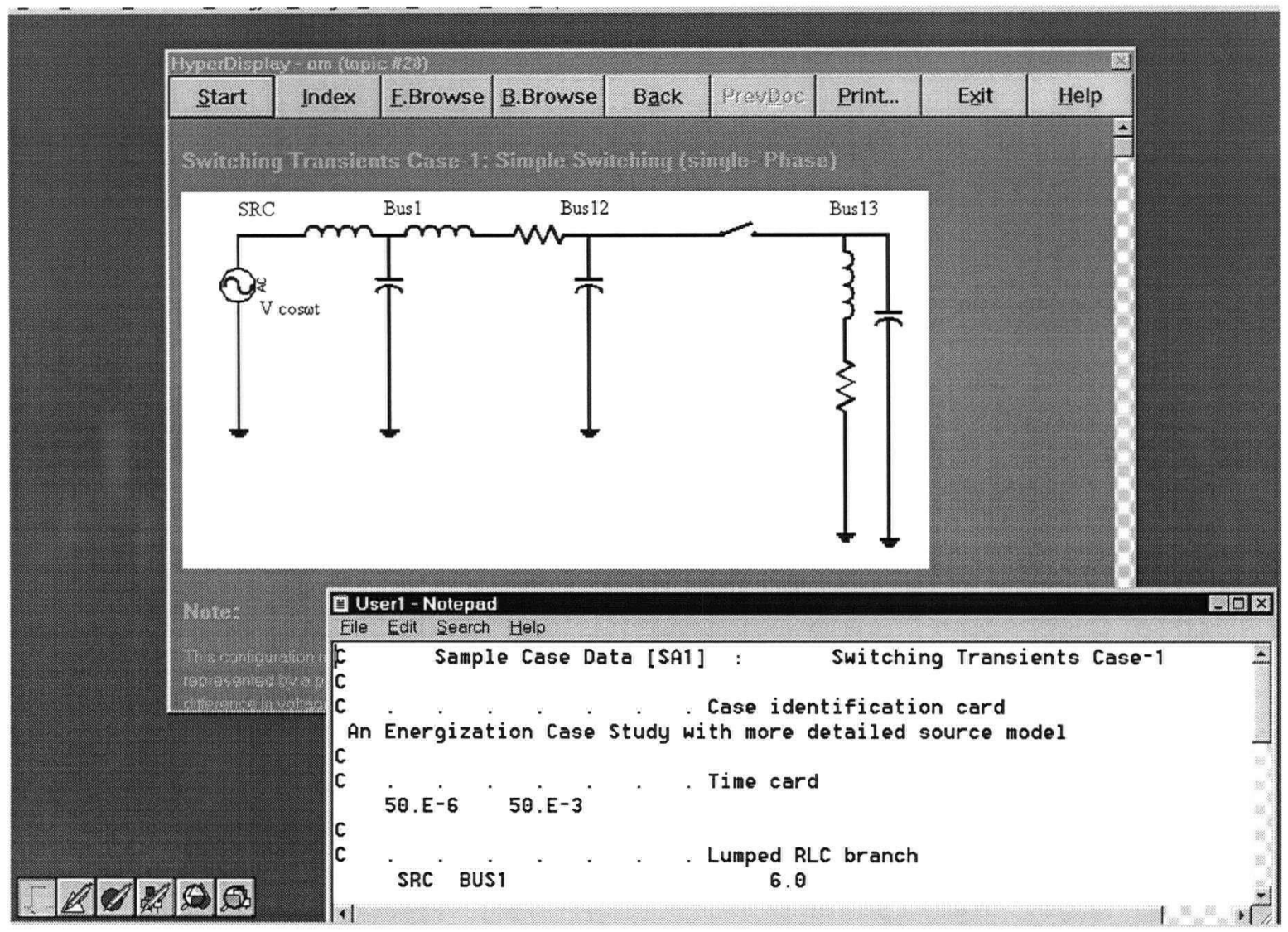

Figure 2.5 The data set of load energization case study

\subsubsection{Data Input and Validation}

\subsubsection{The Data Input Form}

After the user chooses to modify this case data, a form that contains some information about this case data will come up as shown in Figure 2.6. These data are contained in instance objects for each component model used to represent the target power system. Each object has specific attributes and each attribute has a default value. Different instance objects are assembled under class objects that represent various case data sets. For example: 
Class:Load Energization Through Transmission Line Case

Object: $\pi$-Circuit Line Model

Attributes: Resistance, Inductance, Capacitance, and Nodes.

Data validation rules are also classified by objects for efficient application and maintenance.

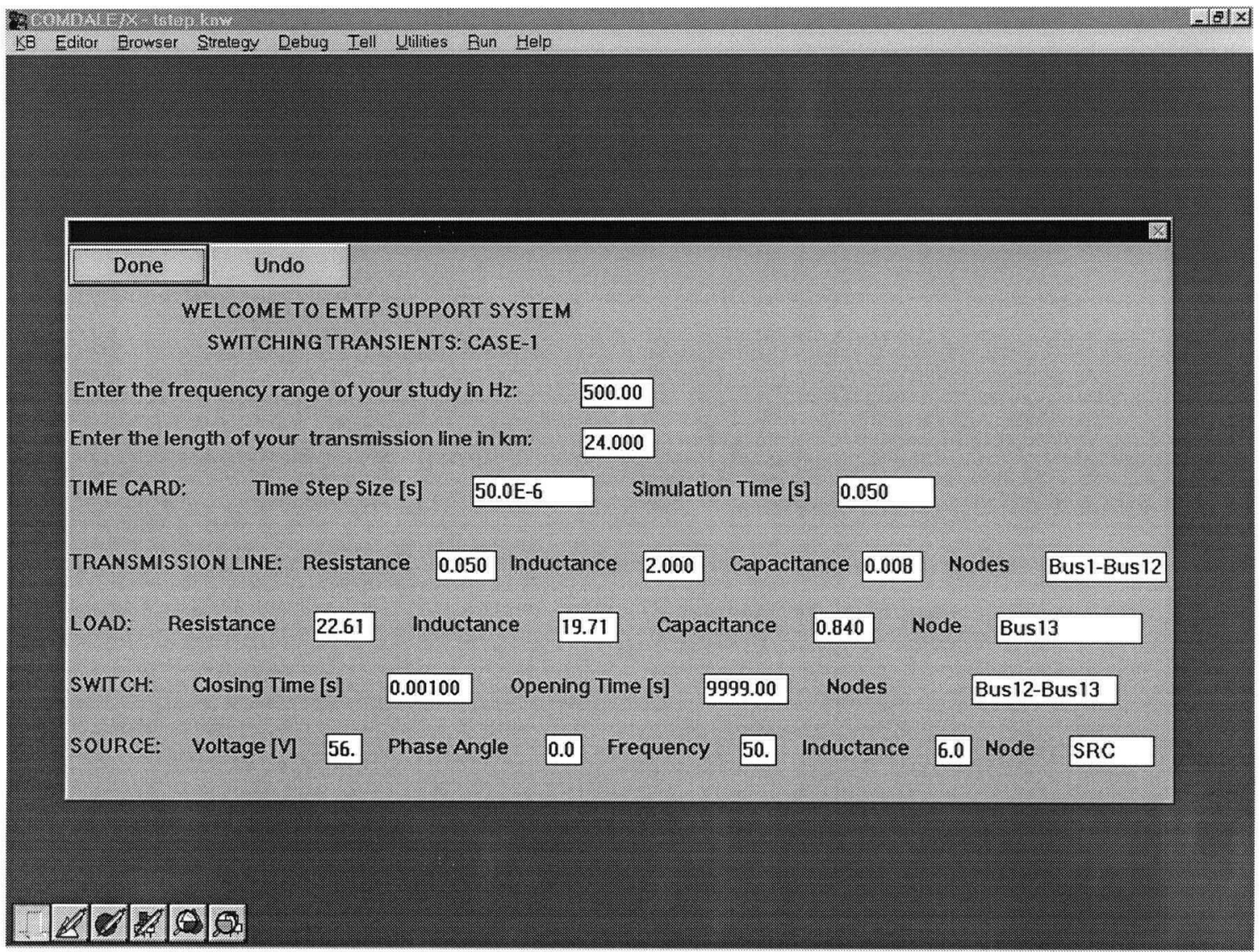

Figure 2.6 Input data form for load energization case study ( $\mathrm{R}$ in $\Omega, \mathrm{L}$ in $m H$, and $\mathrm{C}$ in $\mu F$ ) 


\subsubsection{Rule-Based Data Validation System}

After the user modifies the case data and clicks on the DONE button, the rule-based system will initiate the validation process for this case data. For this simple switching case data, approximately 20 rules were applied to perform the data validation on different objects. The diagnostics from these rules will tell the users about possible inconsistencies of data, so they can correct the case data. Some of these rules are explained as related to their models (objects) as follows:

1. Frequency range \& transmission line length rule

The validity of the $\pi$-circuit line model depends on length $(l)$ of the line as well as on the frequency range of the transient phenomena $(f)$, as shown in equation (1.1).

"If the frequency range of this load energization study is $f$, then the length of the transmission line should not exceed $\left(\frac{10,000}{f}\right)$ for overhead lines".

2. The step size rule

The step size is related to the maximum frequency through the Nyquist frequency. The step size rules will be discussed in more detail in Chapter 3 .

"If the maximum frequency in your system is $f_{\max }$, then use a simulation step size of $\frac{1}{10 \times f_{\max }} "$ 


\section{Transmission line model rules}

In the following cases, the parameters or models are considered incorrect, and the users are instructed to check the parameters.

i) "If the line model parameters ( $L$ and $C$ ) are equal to zero, then this is not a line model".

ii)"If the characteristic impedance of the transmission line $z=\sqrt{(R+j \omega L)^{\prime}(j \omega C)} \approx \sqrt{L^{\prime} C}$ is greater than $1000 \Omega$ or less than $200 \Omega$, then the surge impedance of the line is incorrect if it is an overhead line".

iii) "If the wave speed $c=1 /(\sqrt{L C})$ of the line is greater than $300,000 \mathrm{~km} / \mathrm{s}$ or less than $250,000 \mathrm{~km} / \mathrm{s}$ then the wave speed of the line is incorrect".

The other rules will perform similar sanity checking for the other objects of the case data instance, and the rule-based system will guide the user to select the proper values for each object attribute. Then, the support system will save these values to a file to be converted later to an EMTP format.

When the user is finished with this case data, the support system will activate the EMTP to run this case data as shown in Figure 2.7. 


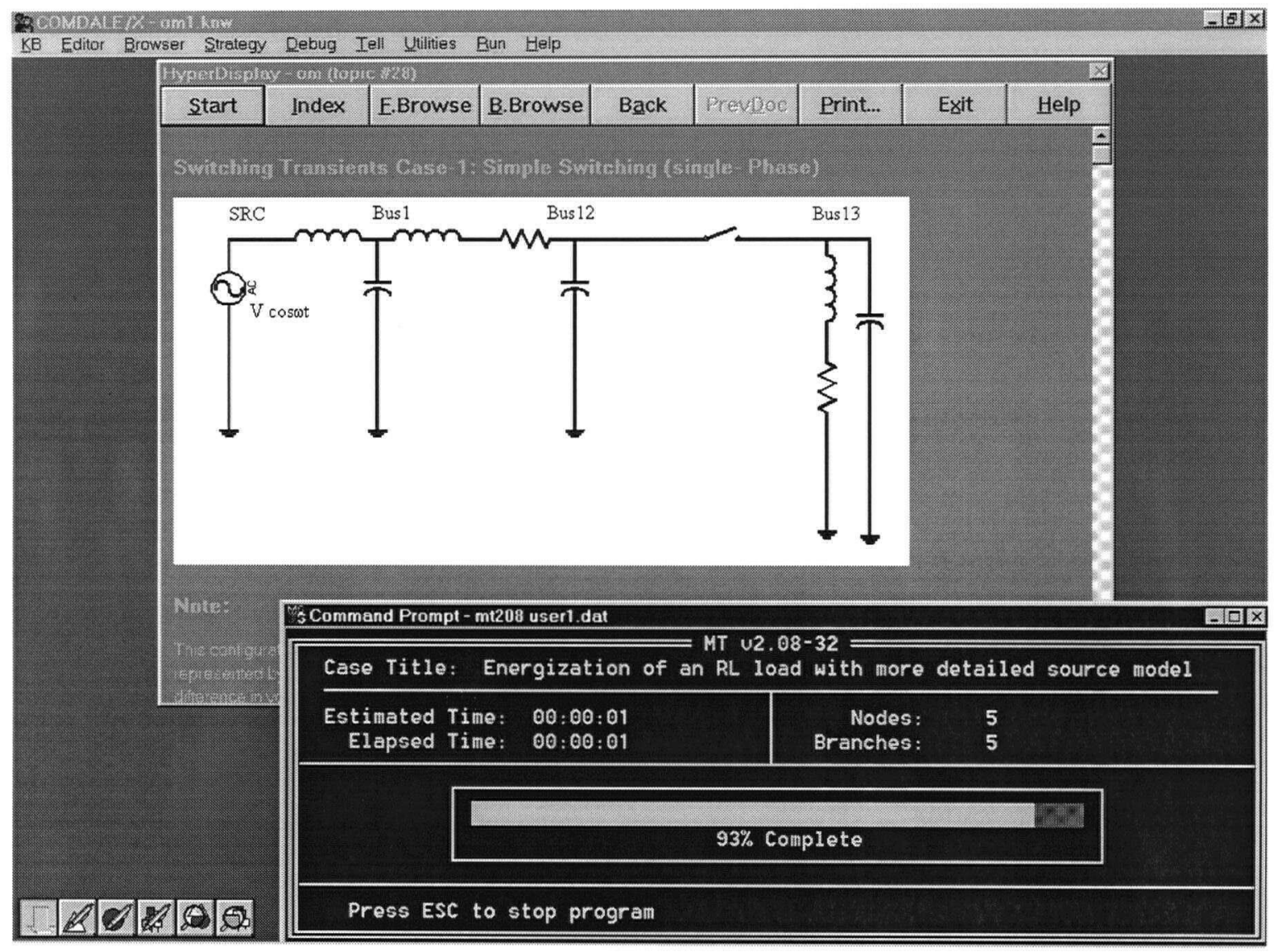

Figure 2.7 The support system running the EMTP

\subsection{Results Evaluation}

This section describes a new method to evaluate the results of EMTP simulations. This method provides a quick check for EMTP results based on parameter variations. In this section, the step size is selected to explain this methodology. This is because the selection of the step size is very important for the accuracy of EMTP simulation results. The step size must be sufficiently small to maintain accuracy and it should be as large as possible in order to provide high simulation efficiency. 
Other parameters such as the load variations were considered in [71] for our results evaluation process. These variations have been applied to the load elements $(R, L$, and $C)$ in order to see how these elements can affect the output voltage waveform.

\subsubsection{Characteristic Parameters}

We selected some features as an example, to evaluate the output waveform of the modified case. More practical features for switching surge studies are discussed in Chapter 3. The two parameters chosen for this particular case study are the maximum voltage amplitude of the first peak $\left(V_{\max }\right)$, and the time at which it crosses the zero voltage axis for the first time $\left(T_{z e r o}\right)$. Figure 2.8 shows a typical energization output waveform and the characteristic parameters that are used to evaluate the results. The overall simulation time for this case is 50 $\mathrm{ms}$, however only the time portion to $5 \mathrm{~ms}$ is shown in Figure 2.8 for comparison purposes.

\subsubsection{Step Size Variations}

In this case study, we consider the step size variation for the results evaluation process, because the waveform shape for the output is sensitive to the selection of the step size. Figure 2.8 compares the base case results (solid line) for a step size of $50 \mu \mathrm{s}$ with the results for a step size of $100 \mu \mathrm{s}$ (dashed line). From Figure 2.8, we can observe how the waveform of the modified case data deviates from the base case. 


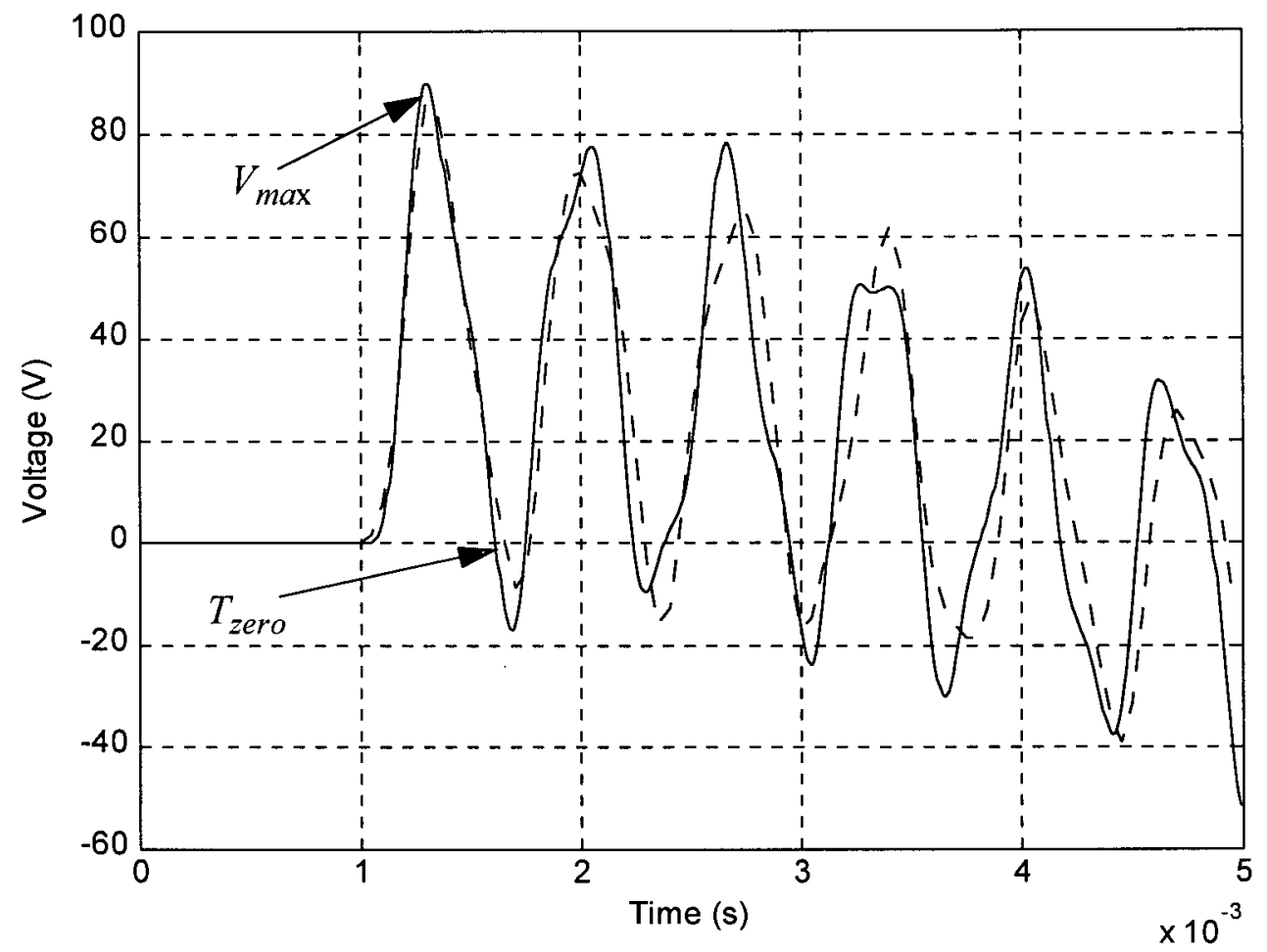

Figure 2.8 Characteristic parameters and case study comparison for step size of $50 \mu \mathrm{s}$ (solid) and $100 \mu \mathrm{s}$ (dashed)

\subsubsection{Fuzzy Logic Analysis}

In this case study, we consider upper and lower limits for the characteristic parameter change from the base case as follows:

$$
\begin{aligned}
& -10.0<\Delta V_{\max }<10.0 \text { in } \mathrm{V} \\
& -1.0<\Delta T_{\text {zero }}<1.0 \text { in } \mathrm{ms}
\end{aligned}
$$


These upper and lower limits were chosen based on experience gained by running many simulations with different step sizes [71].

From the observation of the simulation results, we could declare that the waveform is unacceptable if the changes in the maximum voltage amplitude of the first peak $\left(\Delta V_{\max }\right)$ and the change in time of zero crossing $\left(\Delta T_{z e r o}\right)$ of the modified case data exceeds the upper limits or if they go below the lower limits.

For the application of fuzzy logic, we segment the inputs, namely $\Delta V_{\max }$ and $\Delta T_{z e r o}$, into some overlapped regions by fuzzy sets in Figure 2.9, Figure 2.10. These regions correspond to linguistic labels such as "far low for $\Delta V_{\max }$ ". The output of the waveform comparison $W_{\text {index }}$ represents the matching between the base case and the modified case. $W_{\text {index }}$ can be defined by fuzzy sets as shown in Figure 2.11.

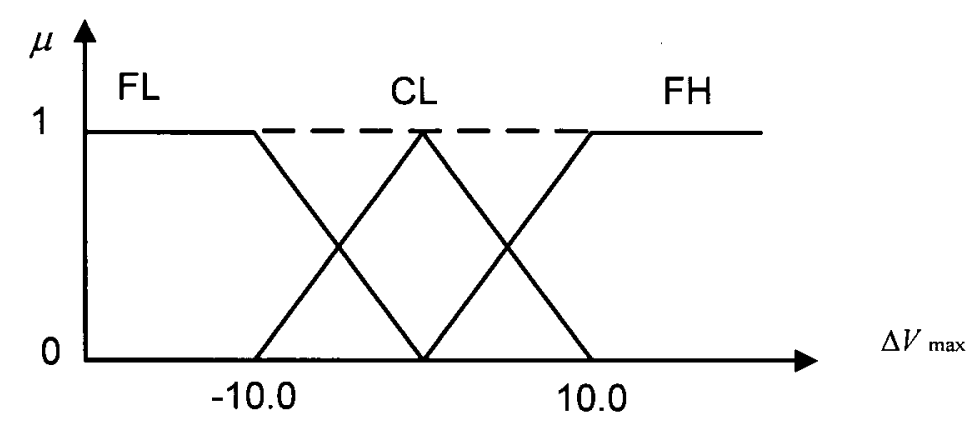

Figure 2.9 The maximum voltage amplitude of the first peak 


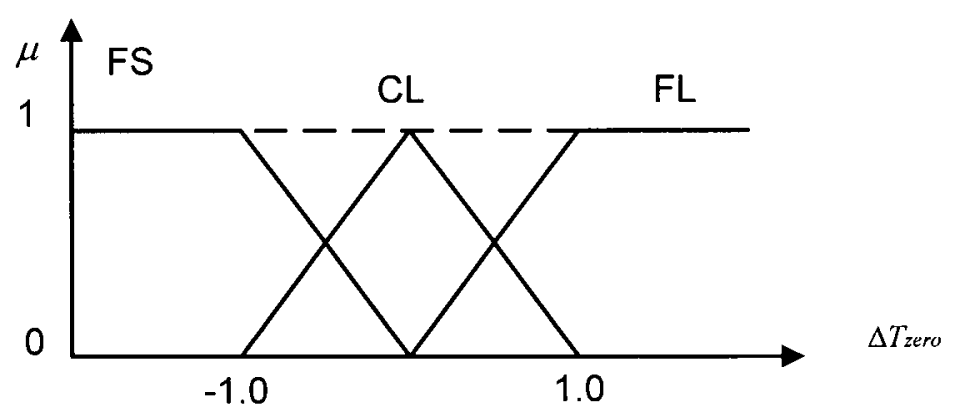

Figure 2.10 The time of zero crossing

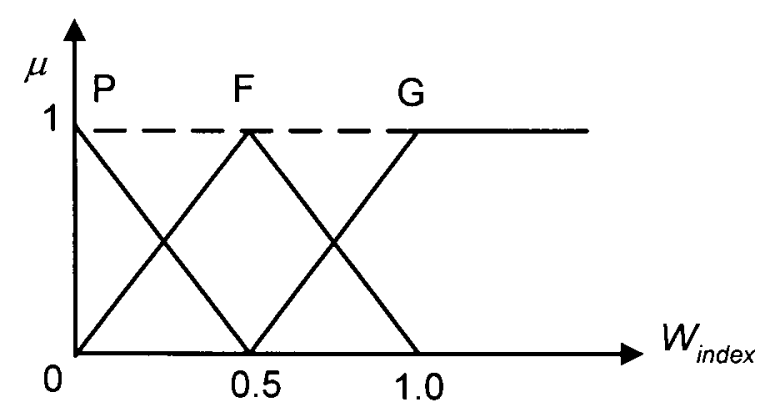

Figure 2.11 Output voltage waveform comparison

The labels in the above figures are as follows:

$\mathrm{FL}=$ Far Low CL $=$ Close $; \mathrm{FH}=$ Far High; $\mathrm{FS}=$ Far Short FL = Far Long; $\mathrm{P}=$ Poor Matching; $F=$ Fair Matching; $\mathrm{G}=$ Good Matching.

Table 2.3 summarizes the rules that are used in the results evaluation process. 
Table 2.3: Results evaluation rules

\begin{tabular}{|c|c|c|c|}
\hline$\Delta \boldsymbol{T}_{\text {zero }}$ & FS & CL & FL \\
\hline FH & $\mathrm{P}$ & $\mathrm{F}$ & $\mathrm{P}$ \\
\hline $\mathbf{C L}$ & $\mathrm{F}$ & $\mathrm{G}$ & $\mathrm{F}$ \\
\hline FL & $\mathrm{P}$ & $\mathrm{F}^{*}$ & $\mathrm{P}$ \\
\hline
\end{tabular}

The rule table reads, for example at the cell marked with asterisk, as:

IF $\Delta T_{\text {zero }}$ is Close

AND $\Delta V_{\max }$ is Far Low

THEN $\quad W_{\text {index }}$ is Fair Matching

There are different fuzzy reasoning methods [69]. For this problem, the popular Mamadnitype fuzzy reasoning mechanism is applied because the number of input-output variables is small.

For example, for a modified case study with characteristic parameters of $\Delta V_{\max }=4 \mathrm{~V}$ and $\Delta T_{\text {zero }}=0.3 \mathrm{~ms}$, and using Mamdani's direct method, the matching index is $W_{\text {index }} \approx 0.7$, which is acceptable. The reasoning process for this given example is shown in Figure 2.12. From this example, we can conclude that the given case has a good similarity to the base case, hence it exhibits reasonably acceptable output. 


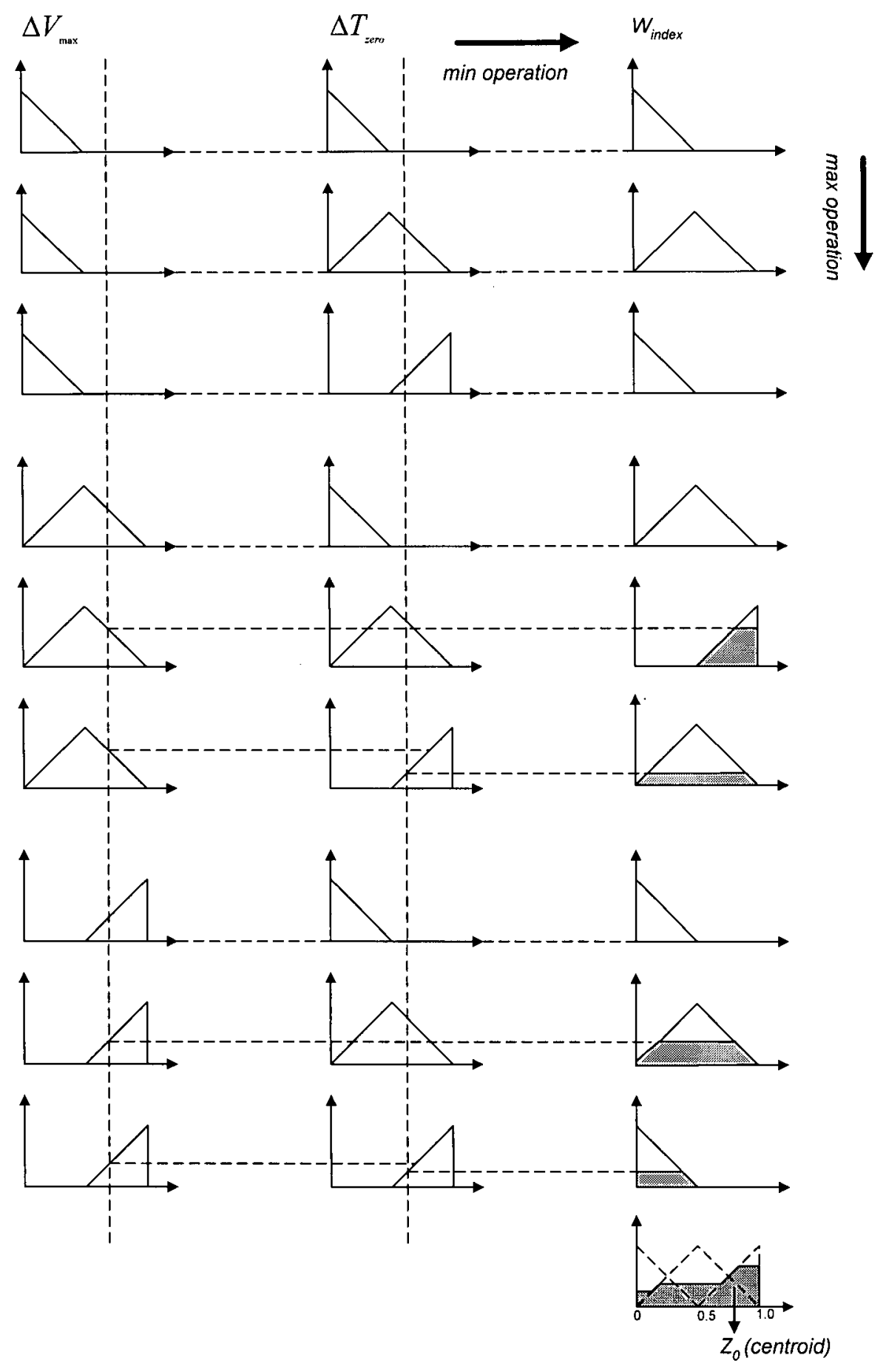

Figure 2.12 Reasoning process 
The fuzzy logic toolbox of MATLAB Version 5.1, was used to implement the results evaluation process for all the derived rules of Table 2.3.

\subsection{Summary}

This chapter has described an EMTP intelligent support system which allows the users of the EMTP to choose the closest case data from a case database that contains different types of simulation case studies. Based on this data, the users can modify their case to meet their particular targets. Also, the data validation and results evaluation process has been applied to a load energization study. The results evaluation process of the support system will advice the user about the acceptable range of the results obtained from the simulation.

The next chapter will present the development of a more realistic knowledge base for switching transient surges. The introduced knowledge base will help in selecting the proper model of representing power system components in the EMTP according to the problem specifications. It will help the user in checking the validity of the data used to represent the simulated transient phenomena before simulation. Also it will help in the evaluation of the results of the EMTP simulation, by using the knowledge of the phenomena being simulated. 


\section{Chapter 3}

\section{Switching Surge Transients}

\subsection{Introduction}

This chapter presents a knowledge base for switching transients overvoltages, and more specifically, for transmission line switching. A practical case of line energization is also presented in this chapter as a base case. The objective is to provide practical rules and modelling suggestions to evaluate switching transients simulations. These rules will be extracted by studying the switching transient phenomena in more detail.

The chapter begins by introducing a summary of different types of overvoltages in general. Section 2 then focuses on the switching overvoltages. This section describes briefly the origin and characteristics of switching overvoltages, the parameter that influence the switching overvoltages and means for limiting them. Section 3 describes a practical case of a three-phase 
transmission line energization. Section 4 then provides some modelling suggestions for the use of different EMTP models.

\subsection{Switching Surge Transients-Background}

With the increasing operating voltage of transmission systems, switching surge overvoltages determine the insulation design rather than lightning overvoltages. The insulation level required to withstand the switching surge overvoltages can have significant influence on the cost of transmission systems. Therefore, an accurate estimation of the switching overvoltages under various conditions of operation is an important factor for the design of transmission systems [1][13].

\subsubsection{Summary of Different Classes of Overvoltages}

Overvoltages in power systems can be produced by a wide variety of phenomena, such as faults, switching operations and lightning strokes. It is not practical to design power equipment to withstand all types of overvoltages. The power engineer must seek a compromise between insulation or protection level and economics. The goals of insulation coordination studies are to select appropriate insulation levels for equipment and to choose protection devices so as to minimize damage and interruptions [17].

Overvoltages that can occur in power systems are classified according to their duration and frequency range:

1. Lightning Overvoltages: These are fast front transients, with front times in the order of microseconds. They are either caused by direct strokes to phase conductors and by back- 
flashovers, or by strokes to earth close to the line [18] [19].

2. Switching Overvoltages: These result from the operation of switching devices, either during normal conditions or as a result of fault clearings. These transients have a duration from tens to thousands of microseconds. They belong to the category of slow front transients. The main operations that can produce switching overvoltages are line energization and re-energization, capacitor and inductor switching, occurrences of faults and breaker openings [1] [2].

3. Temporary Overvoltages: These have frequencies near to or at a harmonic of the power frequency and they are known as long duration power frequency oscillations [20][21].

\subsubsection{Source and Characteristic of Switching Overvoltages}

In general, a switching operation in a power system changes the status of the system from those conditions existing before the switching to those existing after the operation. This will produce transient phenomena. The power frequency voltage before and after the switching operation may be of a different value due to the change in the state of the system. This means that the total amplitude of the overvoltage due to switching may be considered in two parts, namely a transient component which is superimposed on a power frequency component [1].

Switching transients usually show complex waveforms with frequencies in the range of 100 $\mathrm{Hz}$ to $1000 \mathrm{~Hz}$ superimposed on the power frequency. 


\subsubsection{Parameters Influencing Switching Overvoltages}

There are number of parameters of the system and of the operating elements which influence overvoltages. CIGRE Working Group 13.02 [1] presents these parameters as shown in Table 3.1 [1][13], based on an evaluation of a large amount of collected data on closing and reclosing overvoltages. This data is based on Transient Network Analyzer (TNA), computer and field test results. The evaluation shows the relative influence of the large number of parameters on overvoltages. The most important influencing parameters are explained below.

In long transmission lines, the most important factors which affect the power frequency voltages on the line during normal operation, and the increase in voltages during a fault, are the length of the line and the degree of shunt compensation. Both parameters have a major indirect influence on the transient phenomena connected with the initiation or clearing of a fault, as well as with normal switching operations. Even the connection of a transformer at the end of a line can affect the overvoltages. Although one might think that the saturation of the transformer limits the increase in power frequency voltage, that is not so. Instead, the harmonics created by saturation and superimposed on the power frequency typically cause an increase in voltage.

Circuit breakers are less often the direct cause of high overvoltages in power systems than is generally assumed. Their operation is similar to that of an ideal circuit breaker, even when switching high short-circuit currents, switching capacitive current, and to a lesser extent in the case of low inductive currents. They interrupt the current mostly at its natural zero crossing 
point. Overvoltages are, on the other hand, caused by the switching process itself, the characteristic of the system and operation elements, as well as by switching operations and faults immediately prior to it. The circuit breaker itself, together with its closing resistance (the optimum value of which depends on the system characteristic) has a damping effect on switching surges.

There are two characteristics of the system itself which have a major influence on the increase in power frequency voltage and transient phenomena. One of them is the short-circuit power and the other is the system configuration. Low short-circuit power and feeding via a transformer only (inductive source), which occurs in the initial stages of setting up a system, cause much higher switching overvoltages than a high short-circuit power and feeding via transformers and transmission lines (complex system).

As shown in Table 3.1 [1][13], a distinction is made between those parameters inherent in the switched line, the circuit breaker and the supply network. It should be clear that the influence of a single parameter cannot by itself determine the overvoltage, since mutual effects with other parameters are often important. 
Table 3.1:Network parameters influencing the switching overvoltages [1]

\begin{tabular}{|c|c|}
\hline $\begin{array}{c}\text { Network parameter influencing the switching } \\
\text { overvoltages }\end{array}$ & $\begin{array}{l}\text { Influence on total overvoltage } \\
\text { factors }\end{array}$ \\
\hline \multicolumn{2}{|l|}{ 1. Line side parameters } \\
\hline $\begin{array}{l}\text { - Positive and zero sequence inductance, capacitance and } \\
\text { resistance }\end{array}$ & Medium \\
\hline - Frequency dependence of the above line parameters & Medium \\
\hline - Line length & Strong \\
\hline - Degree of shunt compensation & Strong \\
\hline - Degree of series compensation & Medium \\
\hline - Line termination (open or transformer terminated) & Strong \\
\hline $\begin{array}{l}\text { - Presence and degree of trapped charges on the line with } \\
\text { out closing resistors }\end{array}$ & Strong \\
\hline $\begin{array}{l}\text { - Presence and degree of trapped charges on the line with } \\
\text { closing resistors }\end{array}$ & Medium \\
\hline - Corona effects & Minor \\
\hline - Saturation of reactors & Medium \\
\hline - Damping or reactors & Minor \\
\hline \multicolumn{2}{|l|}{ 2. Circuit-breaker parameters } \\
\hline - Max. pole span of contacts & Medium \\
\hline - Dielectric closing characteristics & Minor \\
\hline - Presence of closing resistors & Strong \\
\hline - Value of closing resistor & Strong \\
\hline - Insertion time of closing resistors & Medium \\
\hline - Phase angle at instant of switching & Strong \\
\hline \multicolumn{2}{|l|}{ 3. Supply-side parameters } \\
\hline - Service Voltage & Minor \\
\hline - Service frequency & Minor \\
\hline - Total short-circuit power & Strong \\
\hline $\begin{array}{l}\text { - Frequency-dependent damping factors of transformer and } \\
\text { generators }\end{array}$ & Minor \\
\hline - Inductive and complex network & Strong \\
\hline - Parallel lines to switched line & Minor \\
\hline - Ratio of positive to zero sequence impedance & Minor \\
\hline
\end{tabular}




\subsubsection{Switching Overvoltages in Closing and Reclosing Operations}

Many measurements, and TNA and computer calculations of voltage surges occurring during closing and re-closing of transmission lines were compiled in [1][13]. The results are shown in Figure 3.1. The following parameters were varied:

1. Closing or reclosing

2. Circuit breaker with or without closing resistors

3. Complex or inductive feeding systems

4. Shunt compensation greater or less than $50 \%$. In most of the cases for $\geq 50 \%$ the degree of compensation was approximately $70 \%$, while for the cases of $<50 \%$ mostly no compensation was employed [1].

The average values as well as the maximum and minimum values for the overvoltages were entered into the charts of Figure 3.1.

It can be clearly seen that the highest overvoltages occur during reclosing without closing resistors, when the system consists of a feeding transformer only and no shunt compensation of the transmission line was provided. On the other hand, a simple charging of the line via closing resistors from a complex system, and with shunt compensation of the line, results in the lowest value of overvoltage.

From Figure 3.1 we can see that switching overvoltages are a function of various parameters of the system and the operating elements. At EHV levels, they are limited by shunt compensation, closing resistors, surge arresters and other measures taken during system operation. The 
overvoltages occurring at a particular location in the system must be known in order to decide on the use of particular equipment and its insulation [1].

In conclusion, faults and switching surges are daily occurrences in a system. The switching surges that they cause should not result in further faults or failure of the necessary switching operation. Overvoltages caused by switching on and off of lines, transformers, reactors and other equipment can be effectively reduced by shunt compensation, closing resistors, and surge arresters. Overvoltages which occur at the moment of fault initiation and fault clearing are, on the other hand, a function of the system configuration, the short-circuit power and the method of neutral grounding, and can be controlled or influenced only to a limited extent. Although switching surges are unavoidable, we can nevertheless reduce their frequency of occurrence and magnitude [13].

\subsubsection{Definitions}

In the study presented in Figure 3.1, the following definitions are used [1]:

Inductive source: This expression refers to the supply side network which is fed exclusively through transformers with no lines or cables directly connected to the busbar.

Complex source: The supply side network includes one or more lines or cables directly connected to the busbar.

Variant: A variant is a single examined case in which all system, line and breaker parameters have a specified value, except those which must be varied to find a statistical distribution. 


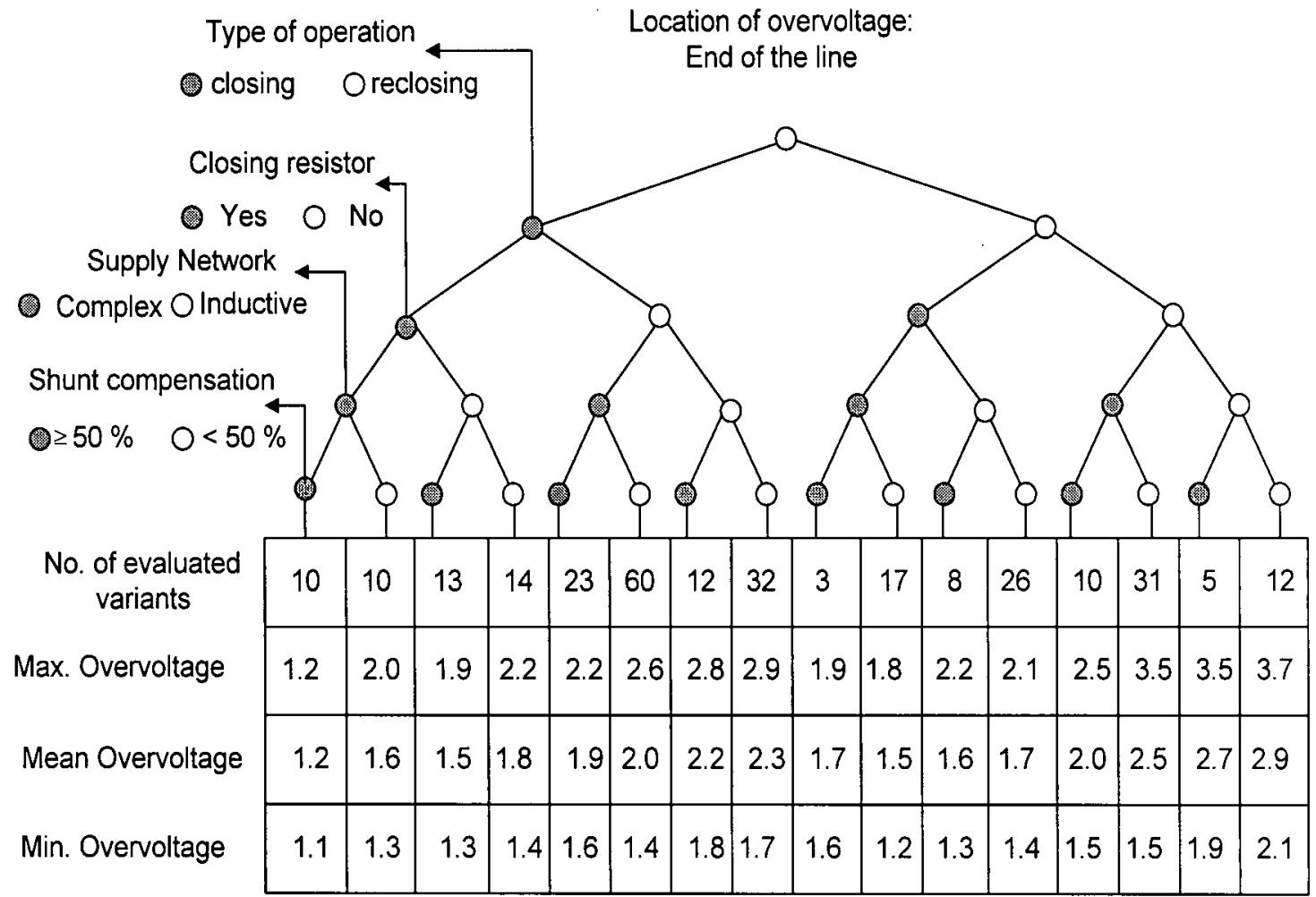

Figure 3.1 Evaluation of overvoltage factors dependent on type of operation and system [1] 


\subsection{Switching Surge During Energization}

This section introduces a switching surge case study of a transmission line energization to be used as the base case for studying the transient phenomena. The network configuration for this case study is shown in Figure 3.2. The data comes from tests on the Jaguara-Taquaril line, which were conducted by the Brazilian utility company CEMIG. Field test data was made available for these test [6] [7][8].

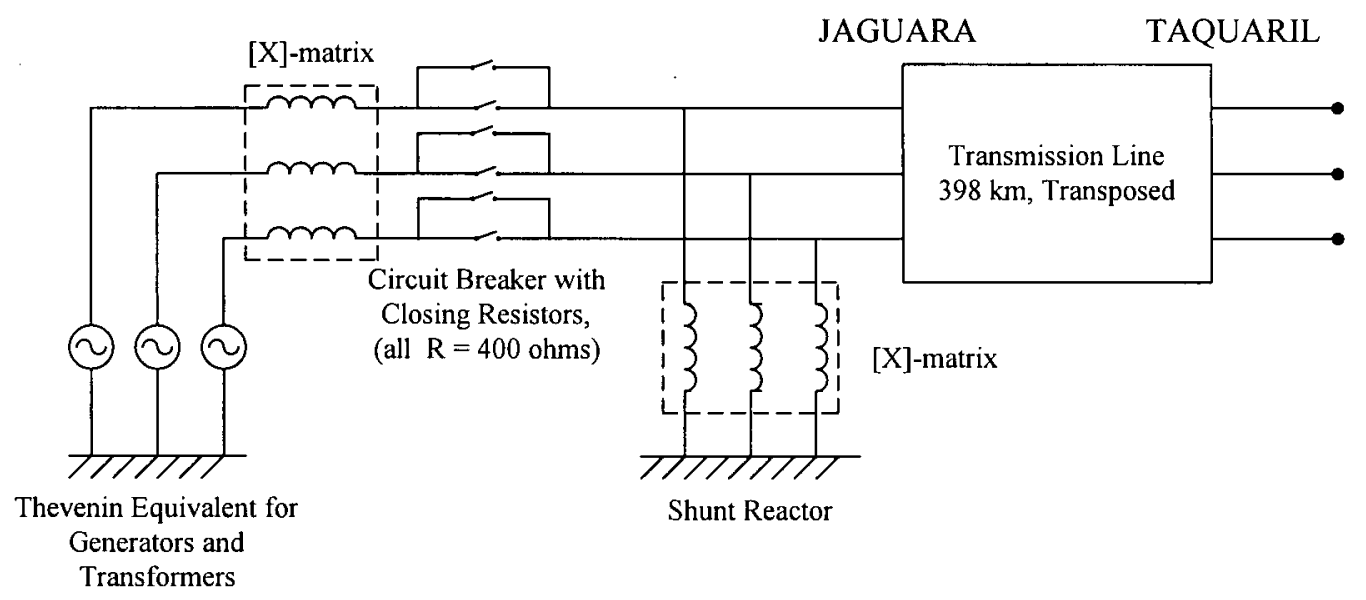

Figure 3.2 Network configuration for switching surge case study

\subsubsection{Network Configuration}

\subsubsection{Feeding Network}

The feeding network was a power plant with synchronous generators and transformers. The subtransient reactances of the generators and the reactances of the transformers could be reduced to a Thevenin equivalent circuit. The Thevenin equivalent impedance matrix was obtained from the positive and zero sequence impedances after conversion to phase quantities 
as shown below:

$$
[X]=\left[\begin{array}{ccc}
77.66 & -22.25 & -22.25 \\
-22.25 & 77.66 & -22.25 \\
-22.25 & -22.25 & 77.66
\end{array}\right] \Omega
$$

These values were referred to the $345 \mathrm{kV}$ side of the transformer.

Immediately before the breaker closing, the voltage on the high side of the transformer was $328 \mathrm{kV}$ (RMS, line to line). These are the values to be used for the voltage source behind the $[X]$ matrix. In the simulation, voltages of 1.0 p.u. were used because the results were to be shown in p.u. of the pre-closing voltage of $328 \mathrm{kV}$. The phase angles were such that the voltage source of phase A passed through zero at $\mathrm{t}=0$, heading for negative values, or:

$$
\begin{aligned}
& \mathrm{v}_{\mathrm{A}}=-1.0 \cos \left(\omega \mathrm{t}+90^{\circ}\right) \\
& \mathrm{v}_{\mathrm{B}}=-1.0 \cos \left(\omega \mathrm{t}-30^{\circ}\right) \\
& \mathrm{v}_{\mathrm{C}}=-1.0 \cos \left(\omega \mathrm{t}-150^{\circ}\right) \quad \text { p.u. }
\end{aligned}
$$

\subsubsection{Shunt Reactor}

The shunt reactor parameter ratings are: $\mathrm{V}_{\text {rating }}=440 \mathrm{kV}, \mathrm{Q}_{\text {rating }}=91 \mathrm{Mvar}, \mathrm{X}_{\text {zero }}=$ $0.35 \mathrm{X}_{\text {pos }}$, wye connection solidly grounded.

From these parameters, the three-phase $[\mathrm{X}]$-matrix was found as:

$$
[X]=\left[\begin{array}{ccc}
1666 . & -461 . & -461 . \\
-461 . & 1666 . & -461 . \\
-461 . & -461 . & 1666 .
\end{array}\right] \Omega \text { at } 60 \mathrm{~Hz}
$$




\subsubsection{Transmission Line}

The sequence parameters of the transmission line were:

$$
\begin{aligned}
& \mathrm{R}_{\text {pos }}=0.0503 \Omega / \mathrm{mile}, \quad \mathrm{X}_{\text {pos }}=0.6021 \Omega / \mathrm{mile}, \quad \mathrm{C}_{\text {pos }}=18.98 \mathrm{nF} / \mathrm{mile} \\
& \mathrm{R}_{\text {zero }}=0.4957 \Omega / \mathrm{mile}, \quad \mathrm{X}_{\text {zero }}=2.061 \Omega / \mathrm{mile}, \quad \mathrm{C}_{\text {zero }}=12.67 \mathrm{nF} / \mathrm{mile} \\
& \text { length }=247.36 \text { miles }=398 \mathrm{~km}(\text { parameters calculated at } 60 \mathrm{~Hz}) .
\end{aligned}
$$

\subsubsection{Circuit Breaker}

The circuit breaker was equipped with closing resistors of $400 \Omega$, which would be in series with the line when the auxiliary contacts were first closed. The closing resistors were then shorted out approximately $7 \mathrm{~ms}$ later as the main contacts closed. The closing times were determined from oscillographs as follows:

$\begin{array}{ccc}\text { Phase } & \text { Auxiliary Contacts } & \text { Main Contacts } \\ \text { A } & 8.45 \mathrm{~ms} & 15.85 \mathrm{~ms} \\ \text { B } & 7.15 \mathrm{~ms} & 14.45 \mathrm{~ms} \\ \text { C } & 8.10 \mathrm{~ms} & 15.10 \mathrm{~ms}\end{array}$

The closing resistors with the resistance values of $400 \Omega$ are represented as switch resistances in the auxiliary contact switches.

The data file used for this case is shown in shown in Table A.1 in Appendix D, while the output file is shown in Table A.2 in Appendix D. 


\subsection{Modelling Suggestions}

This section presents some modelling suggestions for the simulation of line energizations. These suggestions will provide some practical rules which are based on some of the parameters listed in Table 3.1. These modelling suggestions will help the EMTP user to select the proper model and to understand the simulation results better.

\subsubsection{Step Size}

When using the EMTP, the selection of the step size $\Delta t$ is of importance. On the basis of the highest expected frequency, and assuming that ten points would define one period of this frequency $f_{\max }$ with sufficient accuracy, $\Delta t$ is given by [3]:

$$
\Delta t \leq \frac{1}{10 \times f_{\max }}
$$

For the line energization case study, the maximum frequency $f_{\max }$ is expected to be less than 2 kHz. A step size for this case study of $50 \mu \mathrm{s}$ is therefore a reasonable choice. For $\Delta t<50 \mu s$, the results are practically identical with $\Delta t=50 \mu \mathrm{s}$. When the step size $\Delta \mathrm{t}$ is increased to $100 \mu \mathrm{s}$, the results are still accurate. However for $200 \mu \mathrm{s}$, the deviations become noticeable and the results are less accurate [14]. 


\section{The maximum frequency rule:}

"If the maximum frequency in your system is $f_{\text {max }}$ then use the simulation step size of $\frac{1}{10 \times f_{\max }} "$

The travel times for the transmission line are calculated as follows:

$$
\begin{gathered}
\tau_{1}=l \sqrt{L_{1}^{\prime} \cdot C_{1}^{\prime}} \\
\tau_{0}=l \sqrt{L_{0}^{\prime} \cdot C^{\prime}}
\end{gathered}
$$

where $\tau_{1}$ is the positive sequence travel time and $\tau_{0}$ is the zero sequence travel time. In this case, $\tau_{1}=1.36328 \mathrm{~ms}$ and $\tau_{0}=2.06432 \mathrm{~ms}$.

For the existing distributed parameter line model in the EMTP, the step size $\Delta t$ must be less than the travel time of the shortest line in the network. This limitation has been overcome with the new line model presented in more detail in the next chapter. In the case here, the step size $\Delta t$ is less than the travel times $\tau_{1}$ and $\tau_{0}$.

\section{The step size constraint rule:}

"If the step size $\Delta t$ is larger than the travel time $\tau$ of the shortest line in the network, then the new line model should be used for lines with $\tau<\Delta t$ ". 
All line models have some discretization errors, except the lossless line if its travel time is an integer multiple of the step size. If this is not the case, then linear interpolation is used in the EMTP. Linear interpolation is believed to be a reasonable approximation for most cases, since the curves are usually smooth rather than discontinuous. If discontinuity or very sharp peaks do exist, then rounding $\tau$ to the nearest integer multiple of $\Delta \mathrm{t}$ may be more sensible than interpolation [4].

One simple rule that can be applied for checking whether the step size is suitable is to check if no further accuracy can be obtained if the step size is divided by two [3].

\section{Step size accuracy rule:}

"If you want to check the accuracy of the simulation, then divide the used step size by 2 and run the simulation". 


\subsubsection{Transmission Line Models}

The Jaguara-Taquaril case has been simulated using the constant parameter line model, and good results were obtained. This is because there is very little zero sequence current in the results, it is the zero sequence parameters where frequency dependence is pronounced. In cases that have high zero sequence currents, the constant parameter line model is not the right model to be used.

For comparison purposes, the transmission line was also represented with frequency-dependent parameters.

\subsubsection{The Constant Parameter Line Model}

The simulation results for the constant parameter line model are shown in Figure 3.3, Figure 3.4 and Figure 3.5 for the voltages at the receiving end in phase A, phase B and phase C, respectively. For the receiving end at Taquaril, the differences between field test and computer simulation results is larger than at the sending end (not shown here). The exact reason for this difference is not sufficiently known. It could have been caused by the fact that the metering installation at Taquaril was relatively unreliable, consisting of a galvanometric oscillograph coupled to differential amplifiers placed inside a metallic cubicle without having any protection against the heat of the sun [7]. There was difficulty in calibrating the heat-sensitive amplifiers. At the sending end, a more reliable high-speed cathode ray oscillograph was used [7]. 


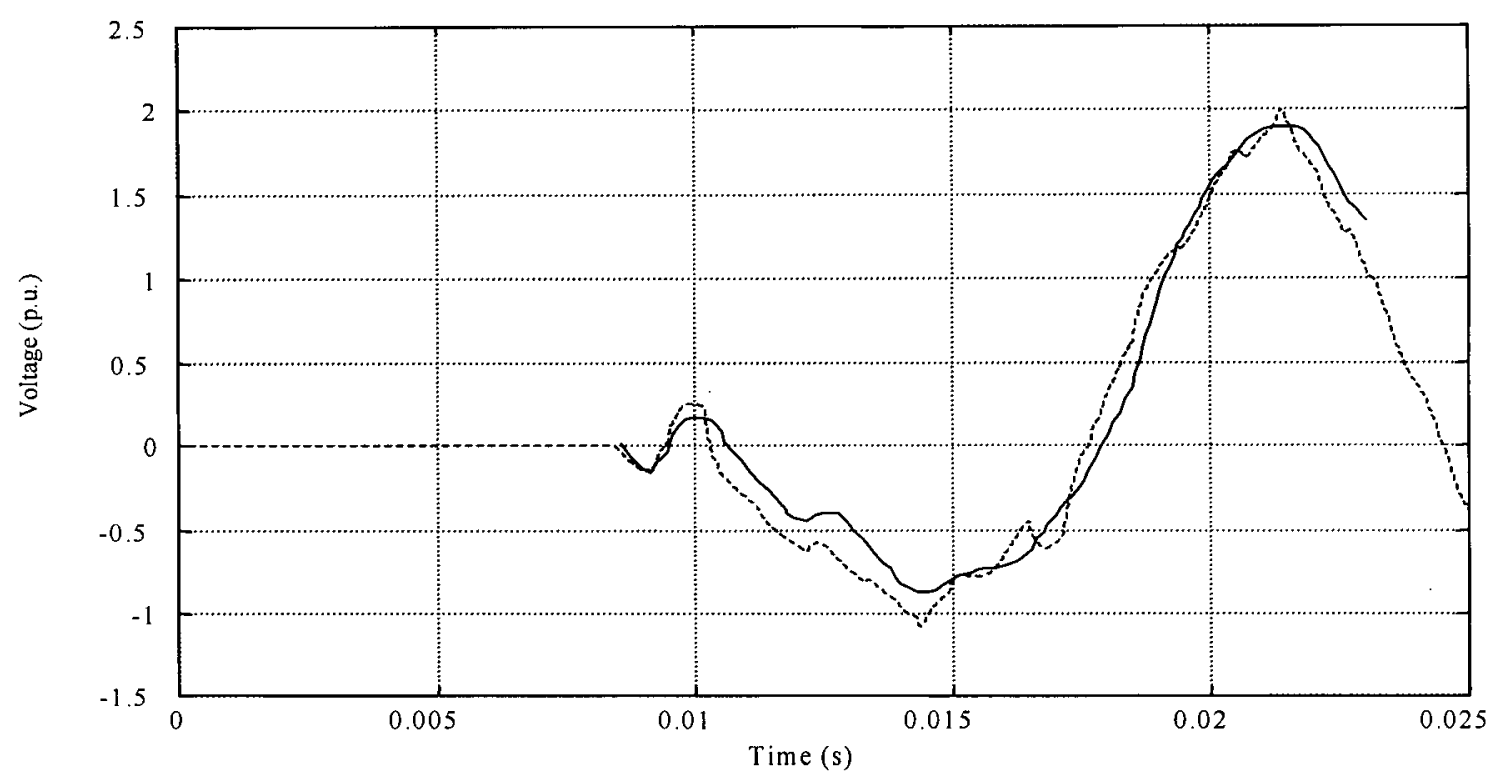

Figure 3.3 Comparison between field test (solid) and constant parameter line model (dashed) in phase $\mathrm{A}$

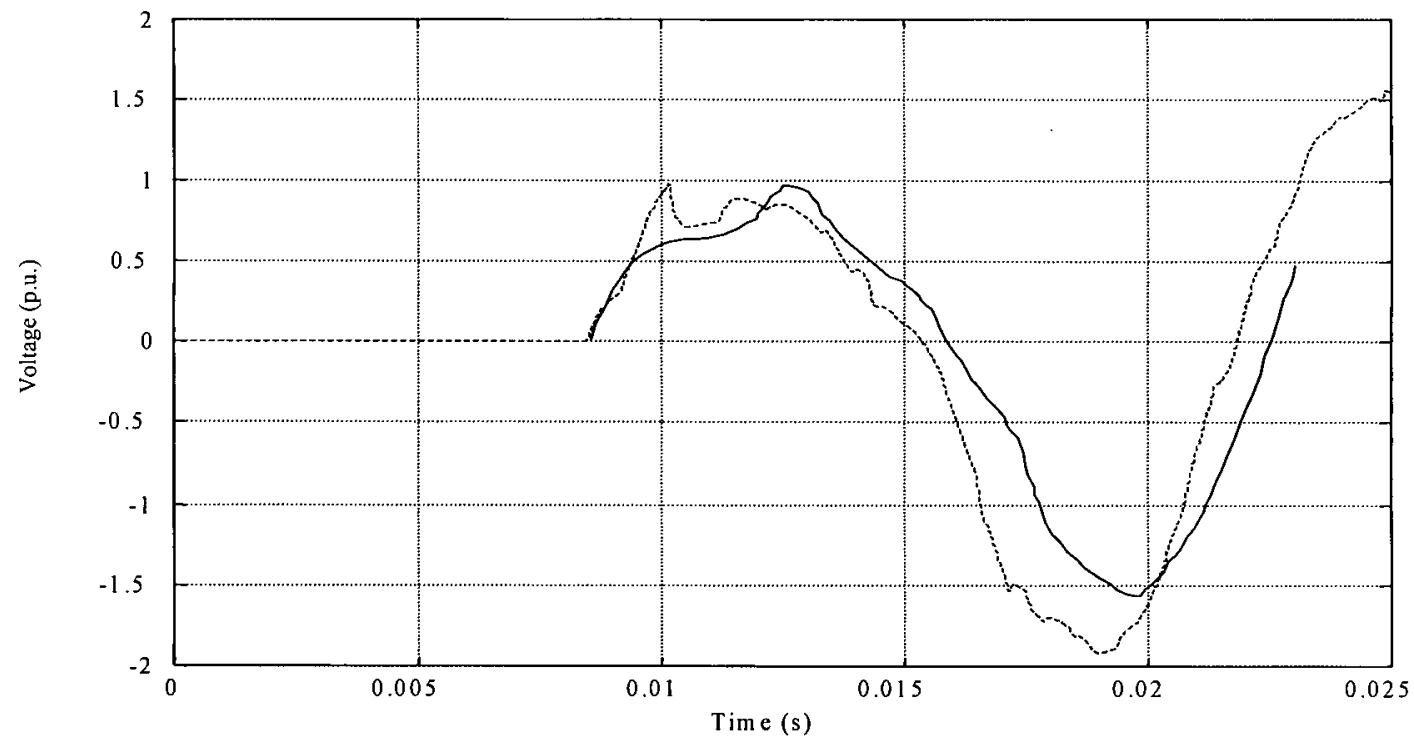

Figure 3.4 Comparison between field test (solid) and constant parameter line model (dashed) in phase $B$ 


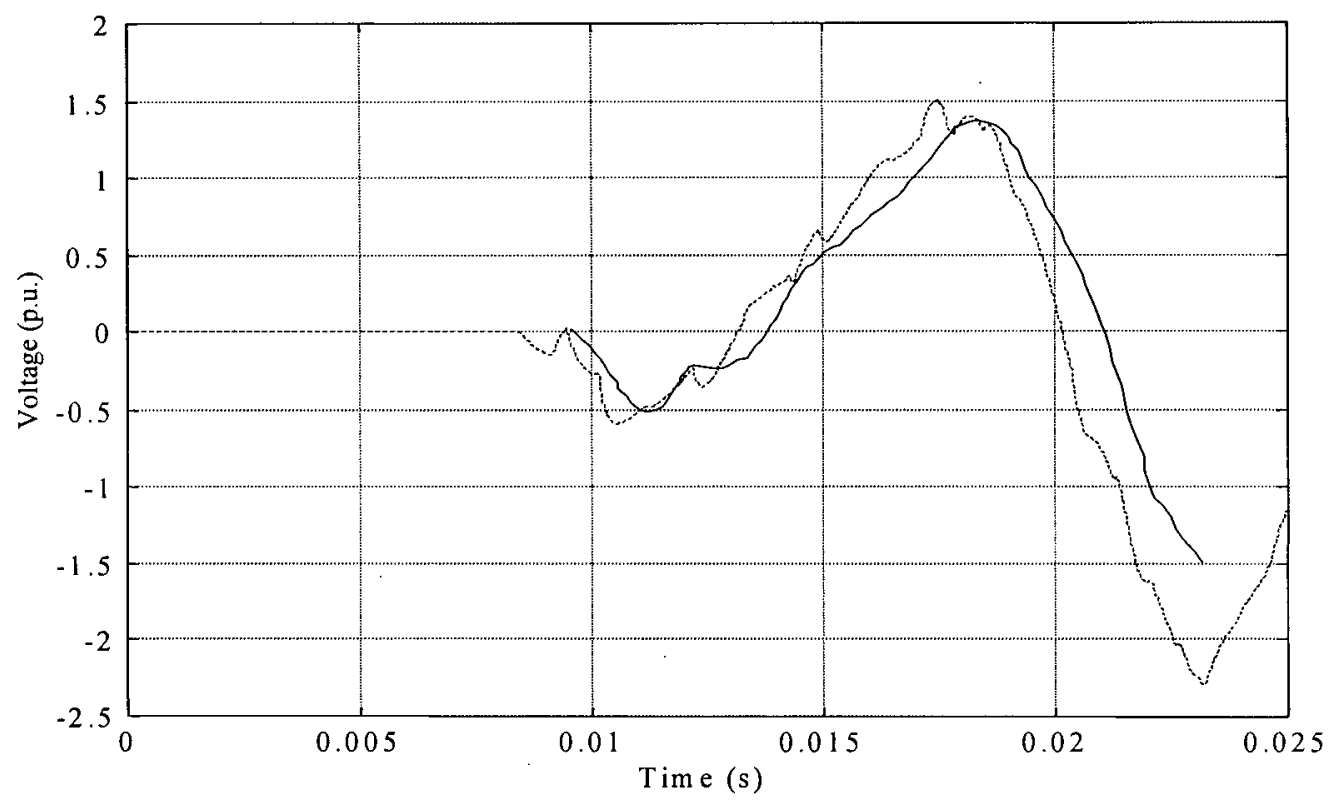

Figure 3.5 Comparison between field test (solid) and constant parameter line model (dashed) in phase $\mathrm{C}$

\subsubsection{The Frequency Dependent Line Model}

Figure 3.6 gives the tower configuration of the line. This geometry and the characteristic of the conductors are needed to calculate the frequency dependent line parameters for both positive and zero sequence. The conductor characteristics were taken from [11], [12].

All the parameters, including series resistance and shunt conductance, are modeled as continuously distributed along the line's length in this line model. It is based on the synthesis by rational functions, in the frequency domain, of the line propagation function and characteristic impedance. These rational approximations correspond to a sum of simple partial fractions in the time domain [4]. 
The EMTP input data file for this line model is produced by the auxiliary program fdData. This file contains the partial fractions expansion of the characteristic impedance and propagation function for the zero sequence and positive sequence mode [10].

The fdData input file for this case is shown in Table A.3 in Appendix D. The EMTP input data file for the case with the frequency dependent line model is shown in Table A.4 in Appendix D.

The simulation results for the frequency dependent line model are shown in Figure 3.7, Figure 3.8 and Figure 3.9 for phase A, phase B and phase C, respectively. 


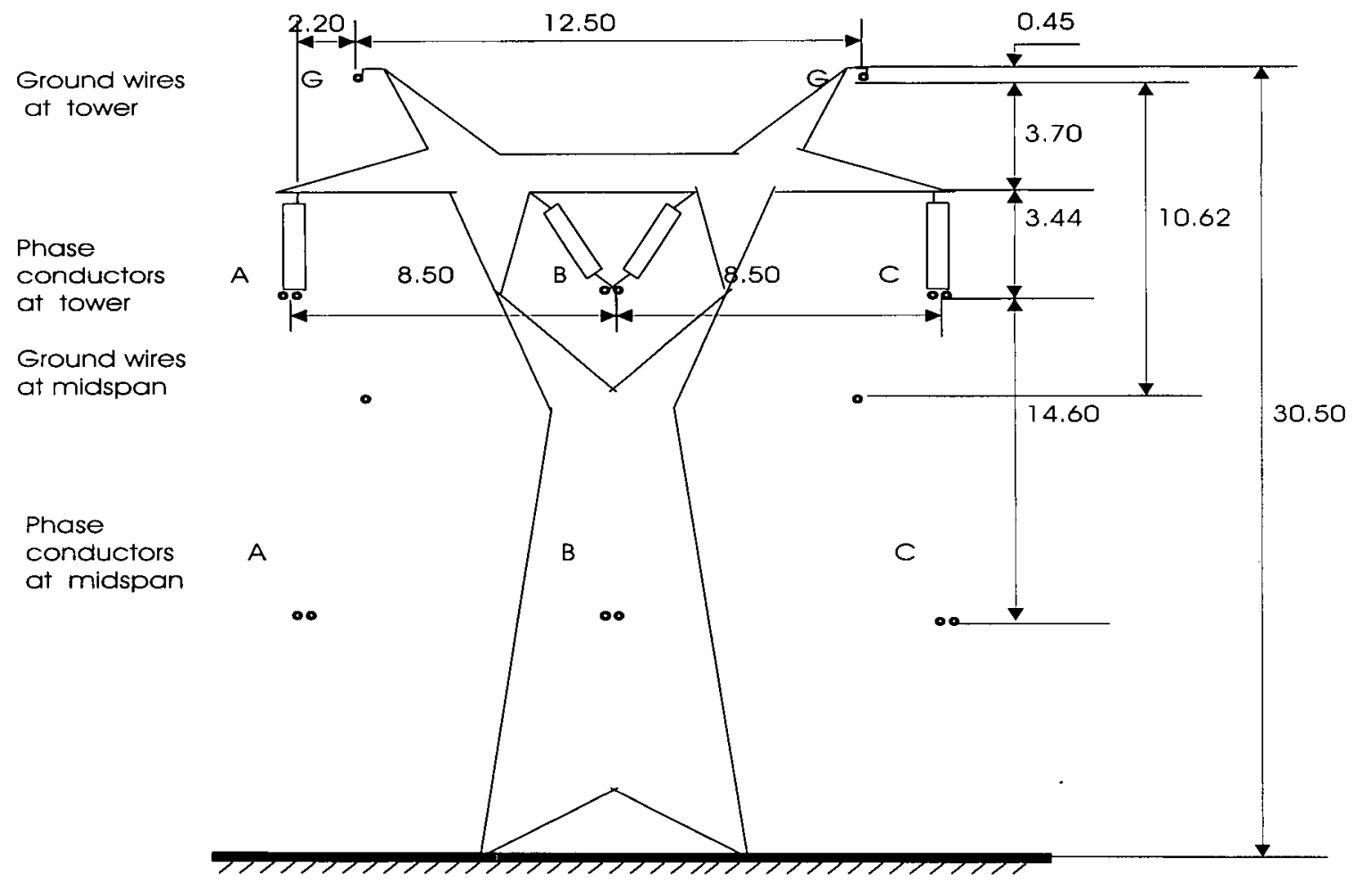

Figure 3.6 Tower configuration of Jaguara-Taquaril line. All measurements in meters, earth resistivity $=100 \Omega \mathrm{m}$ 


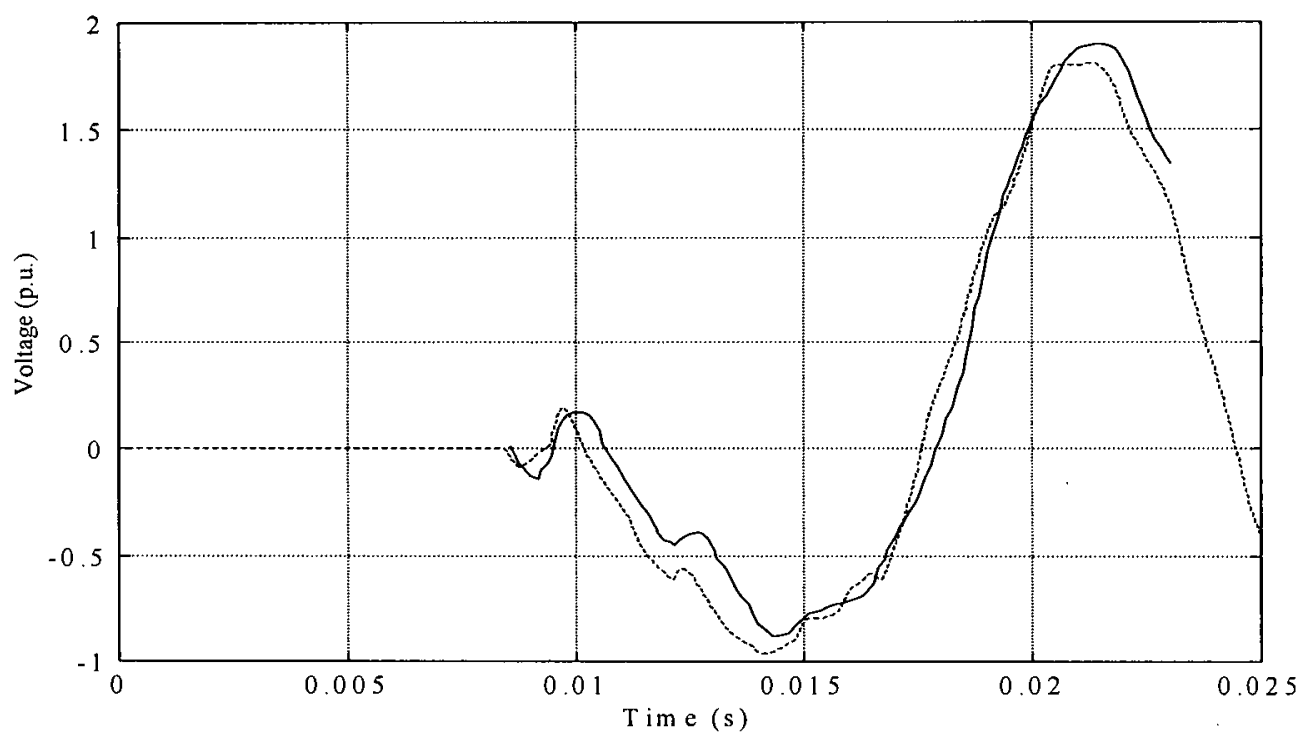

Figure 3.7 Comparison between field test (solid) and frequency dependent line (dashed) in phase A

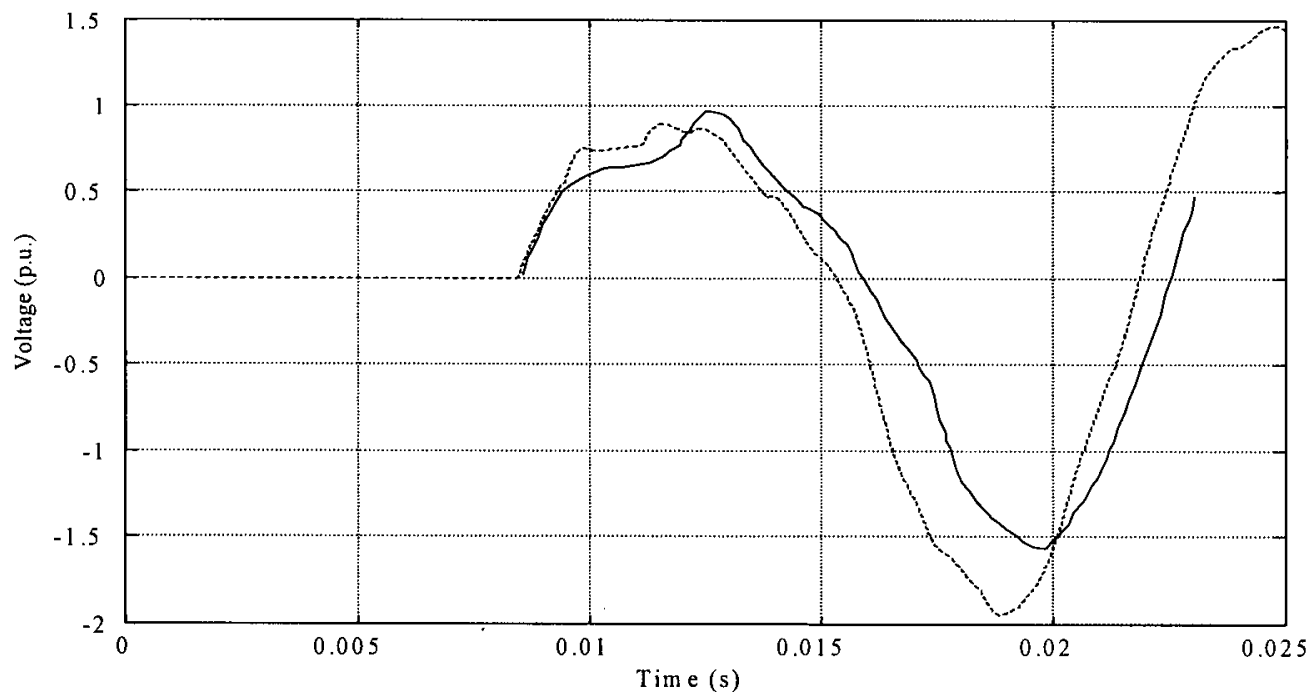

Figure 3.8 Comparison between field test (solid) and frequency dependent line (dashed) in phase B 


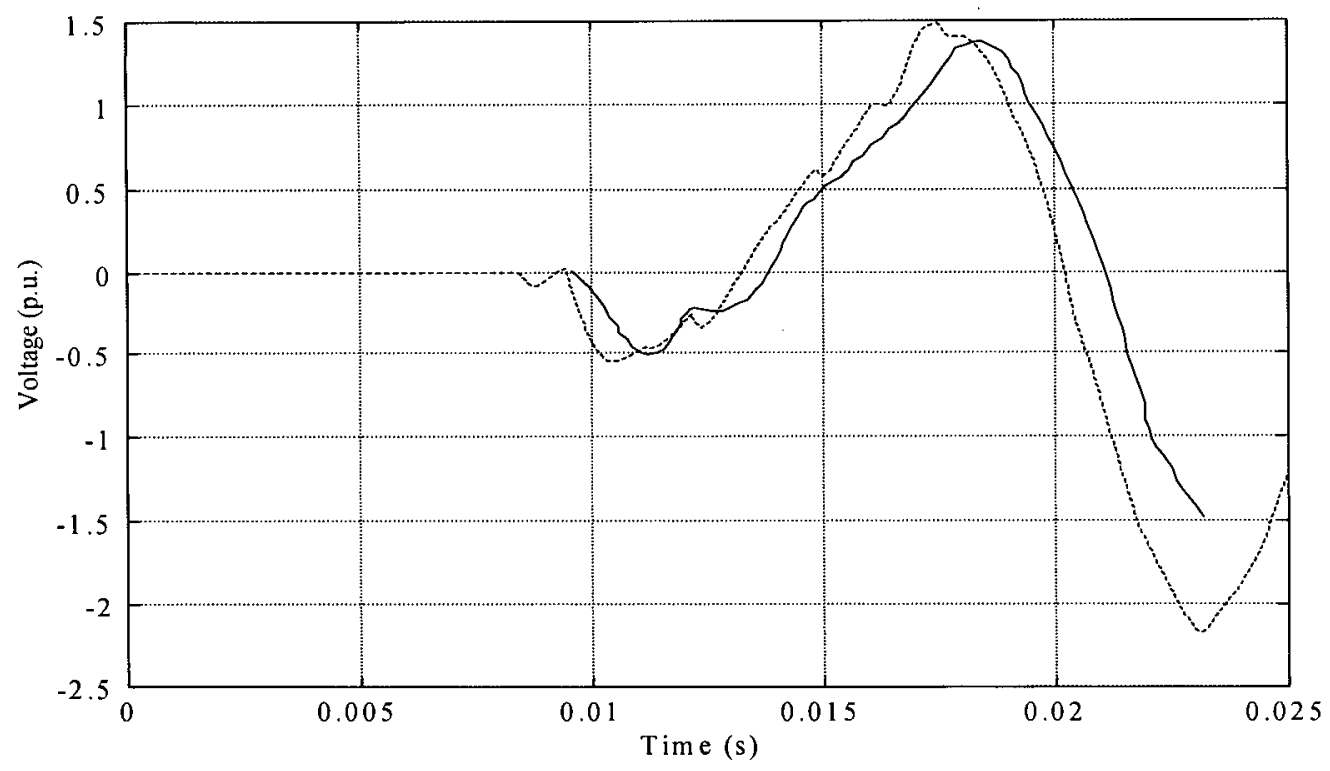

Figure 3.9 Comparison between field test (solid) and frequency dependent line (dashed) in phase $\mathrm{C}$ 


\subsubsection{Comparison Between the Two Line Models}

The simulation results for the frequency dependent line model are compared with those of the constant parameter line model in Figure 3.10, Figure 3.11 and Figure 3.12. From these results we can observe that the voltages at the receiving end are almost identical, except for a $10 \%$ difference in the peak which is caused by a less damped high frequency oscillation in the constant parameter line model. This is because the zero sequence parameters have the strong frequency dependence, but the zero sequence current flowing in the network is small compared to the phase currents. The zero sequence current for this case is shown in Figure 3.13. The currents for the three phases A, B and C are shown in Figure 3.14.

If the zero sequence current is relatively high, then the constant parameter line model will not give accurate results as compared to the frequency dependent line model. This is because the higher frequency damping in zero sequence cannot be accurately represented with the constant parameter line model. 


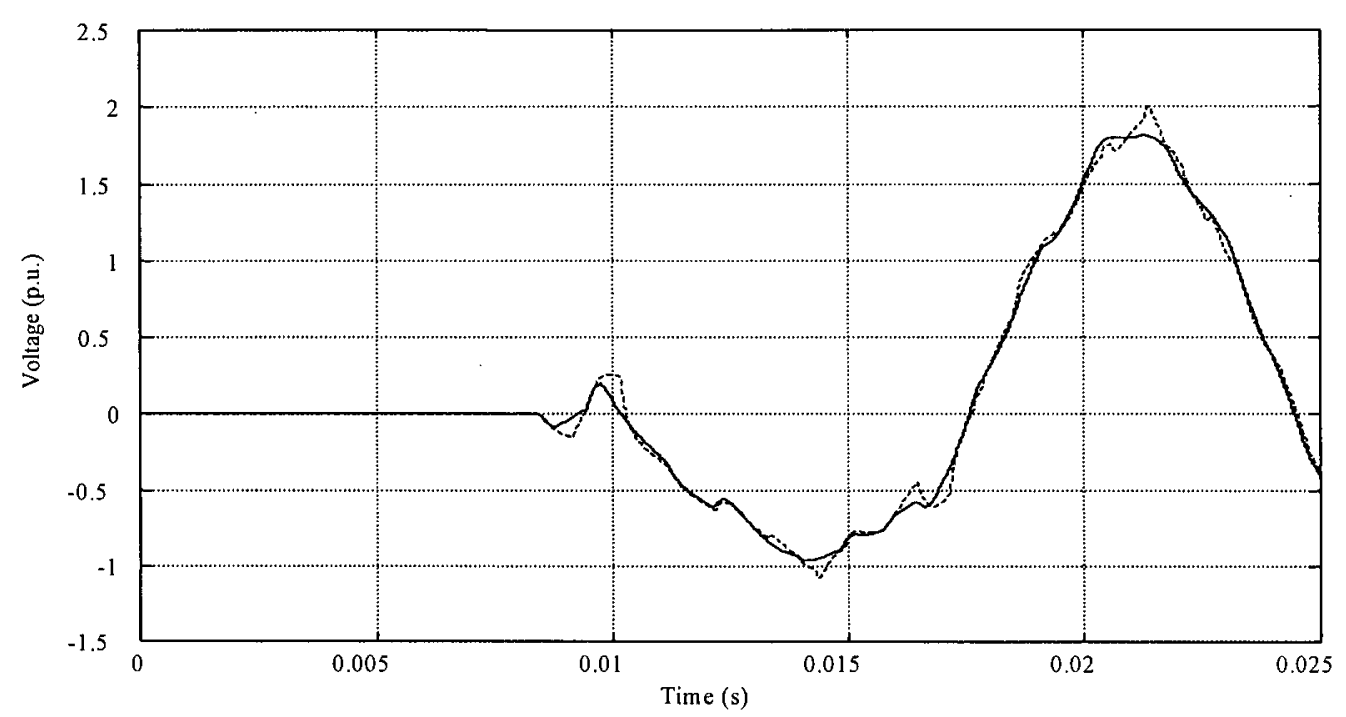

Figure 3.10 Comparison between frequency dependent line (solid) and constant parameter line (dashed) in phase A

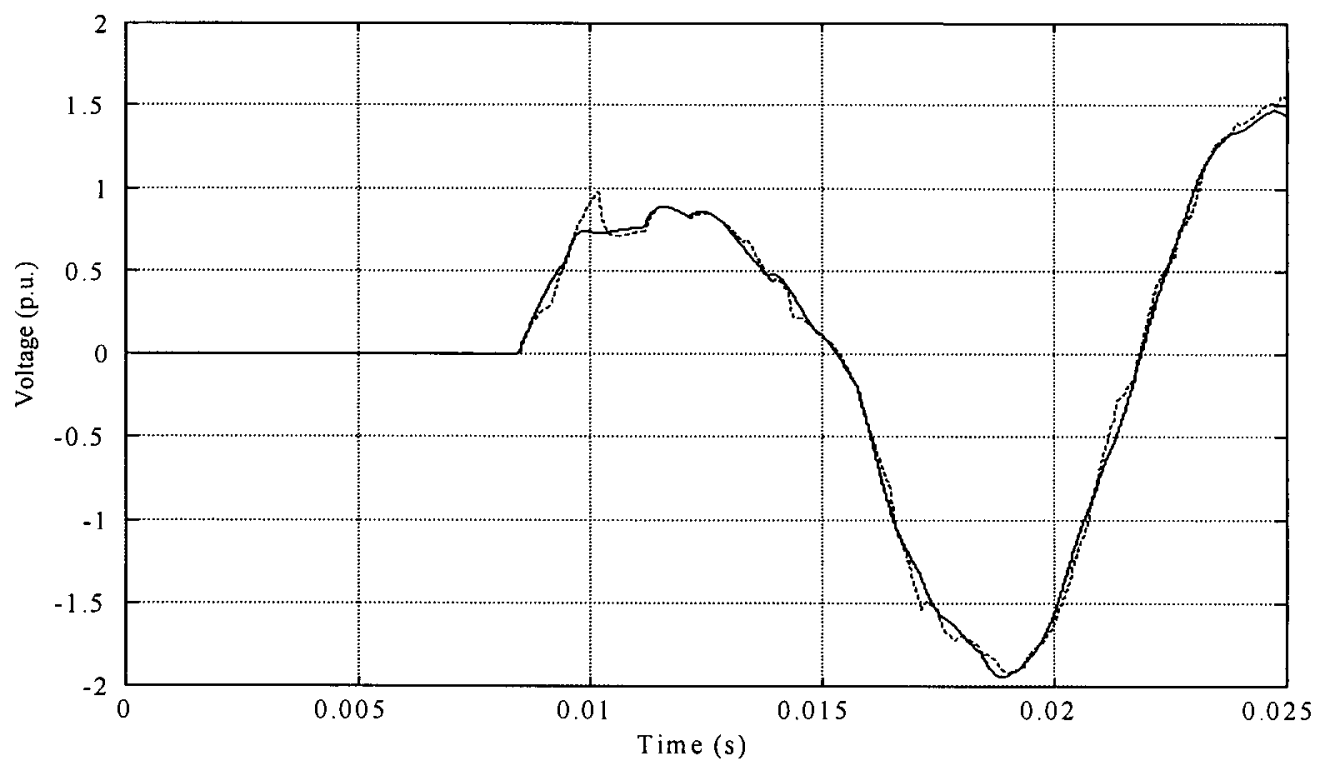

Figure 3.11 Comparison between frequency dependent line (solid) and constant parameter line (dashed) in phase B 


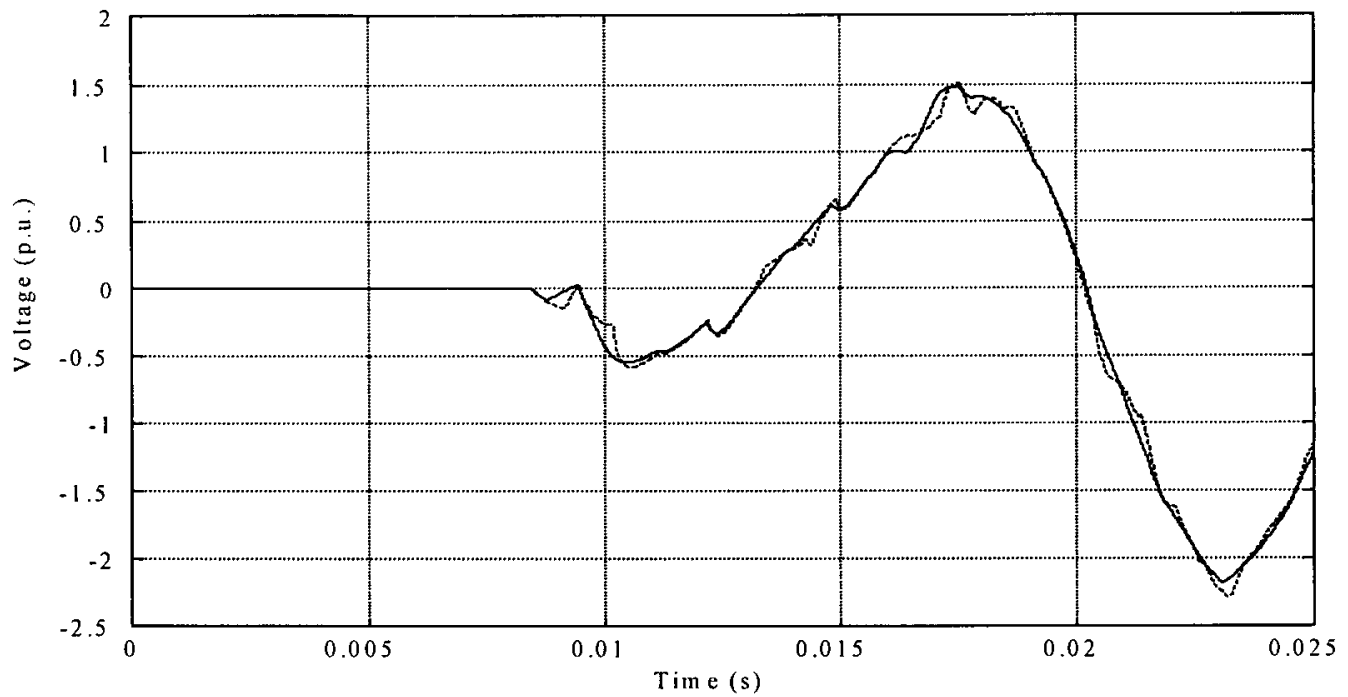

Figure 3.12 Comparison between frequency dependent line (solid) and constant parameter line (dashed) in phase C

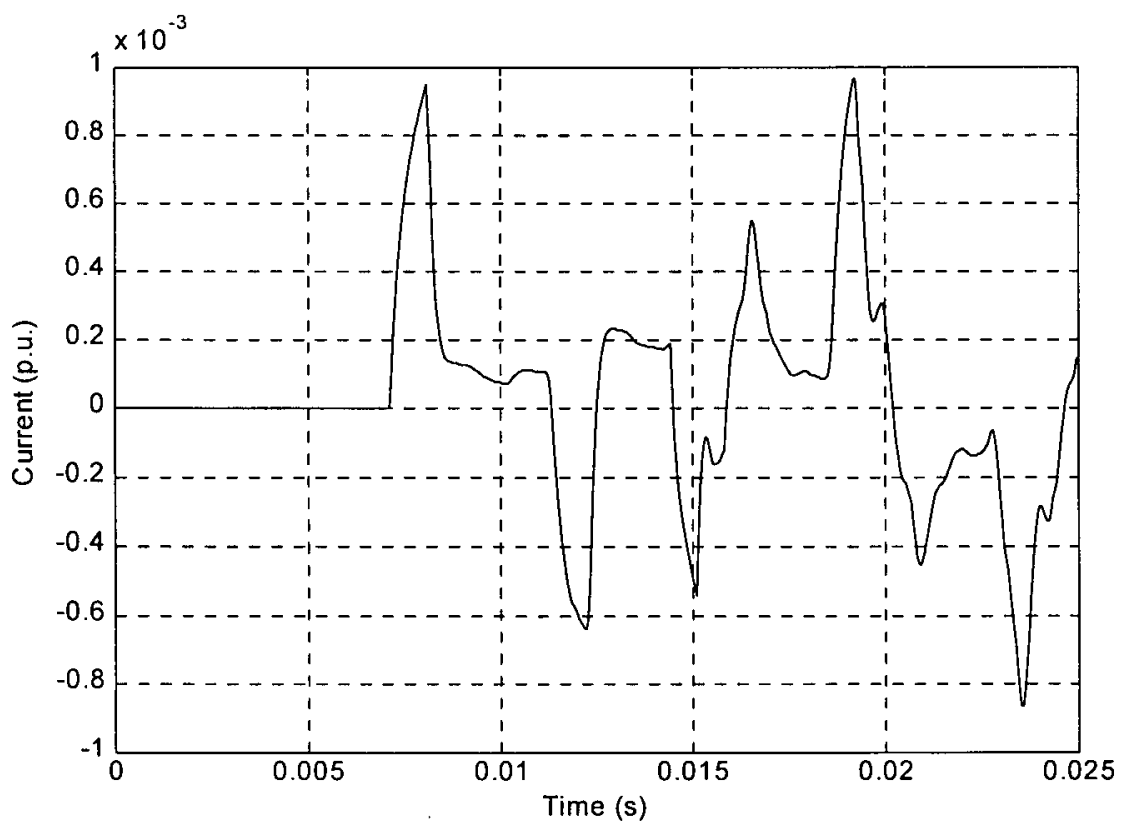

Figure 3.13 Zero sequence current for Jaguara case study 


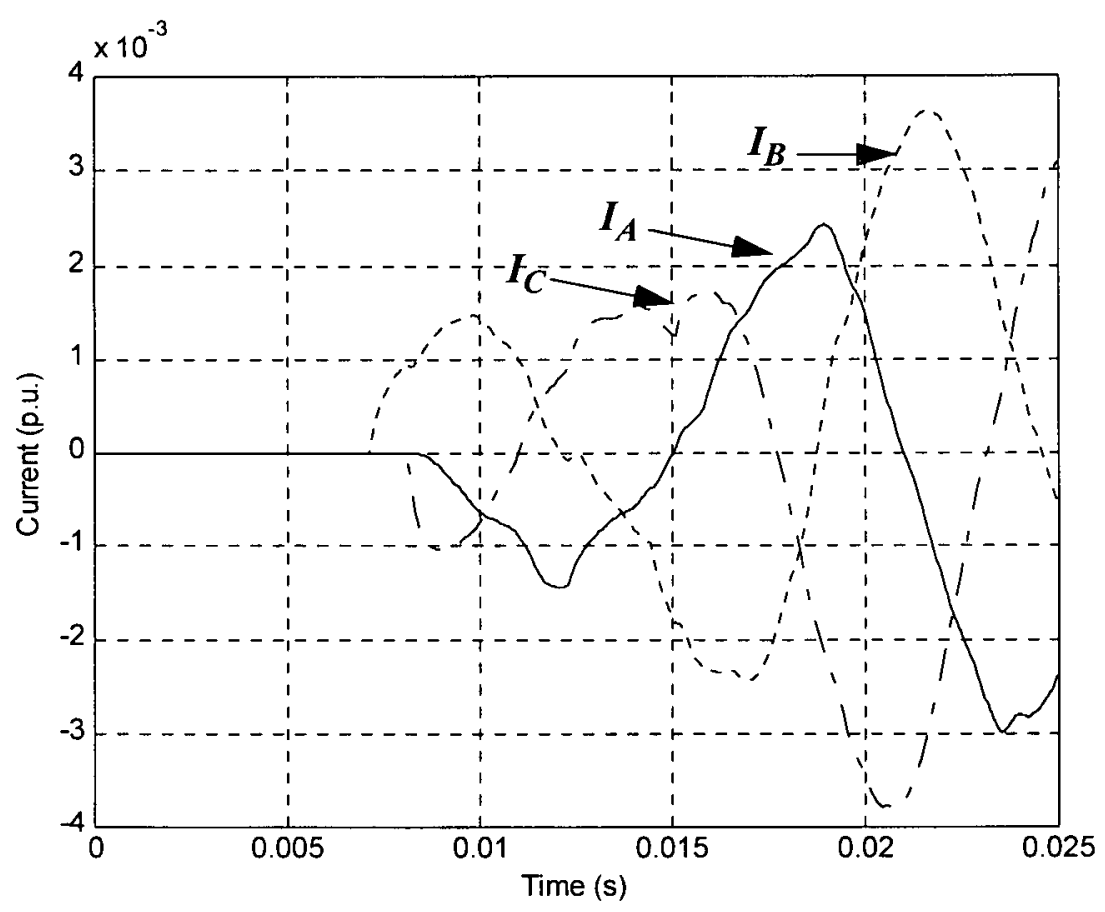

Figure 3.14 Three phase currents for Jaguara case study

\subsubsection{The Single-Phase Energization Case Study}

A case of single-phase energization is presented to show a situation where the results from the constant parameter line model differ more from those of the frequency dependent line model. The simulation results are shown in Figure 3.15 and Figure 3.16 for phases A and B, and C, respectively. The voltages in the unenergized phases $\mathrm{B}$ and $\mathrm{C}$ are identical, and result from coupling effect to the energized phase.

In this case the zero sequence current is equal to the single phase current of phase $\mathrm{A}$. It is shown in Figure 3.17. The case was slightly modified from the three-phase energization case: there was no closing resistors, and the voltage in the feeding network was at the peak value 
when the circuit breaker closed. This produced more higher frequencies than closing at zero voltage.

If we compare this zero sequence current to that of the three phase energization, we can see that for this case the zero sequence current is much larger. This makes a difference in choosing the proper model. If we look at the positive sequence inductance of the overhead line we can see that it is practically constant, as shown in Figure 3.18, while the positive sequence resistance remains more or less constant until the skin effect in conductors becomes noticeable, as shown in Figure 3.19. The zero sequence inductance is shown in Figure 3.20 and zero sequence resistance is shown in Figure 3.21. Both are very much frequency dependent, due to the skin effect in the earth return.

The frequency dependent model includes information about the variation of the parameters with frequency. This is an important consideration when the ground return mode (zero sequence) is involved. In these cases, the frequency dependent line model will give more accurate representation for a wide range of frequencies contained in the transient phenomena, as compared to the constant parameter line model. 


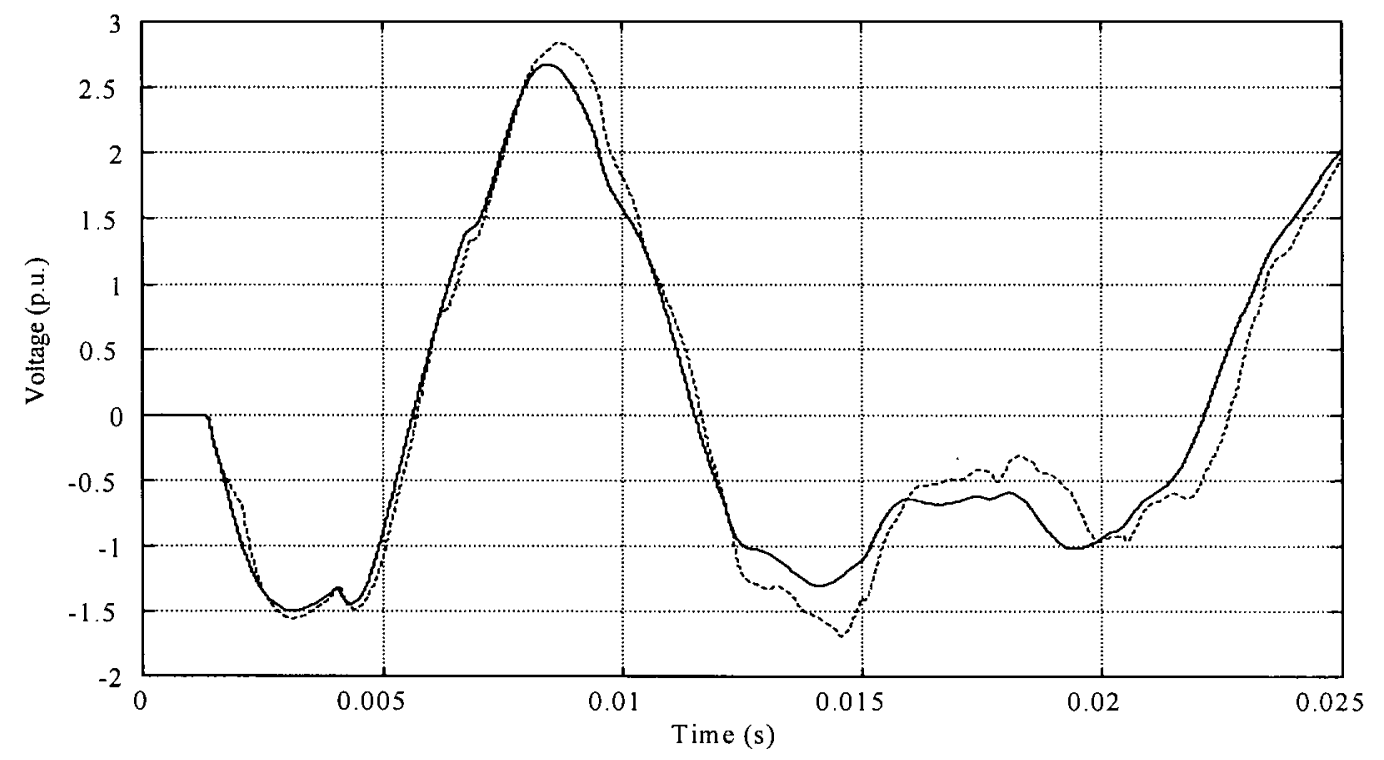

Figure 3.15 Comparison between frequency dependent line (solid) and constant line (dashed) for phase $\mathrm{A}$

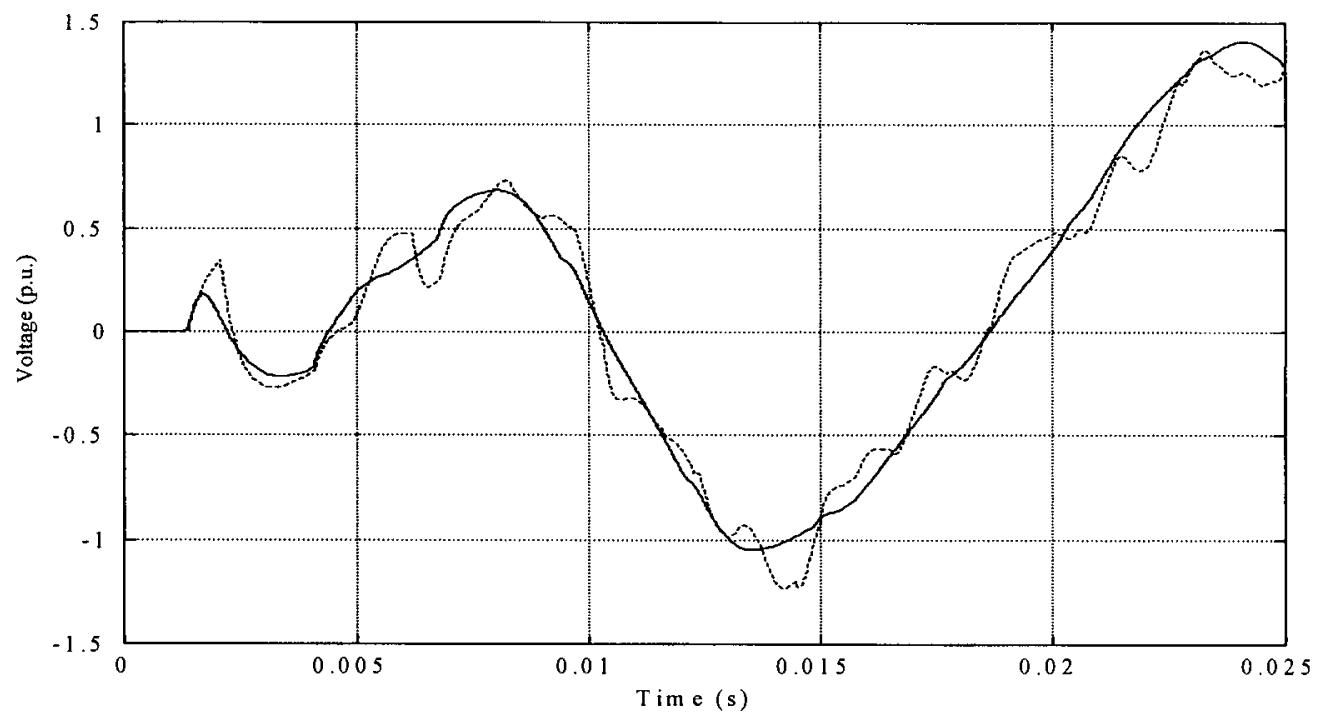

Figure 3.16 Comparison between frequency dependent line (solid) and constant line (dashed) for phase $\mathrm{B}$ and $\mathrm{C}$ 


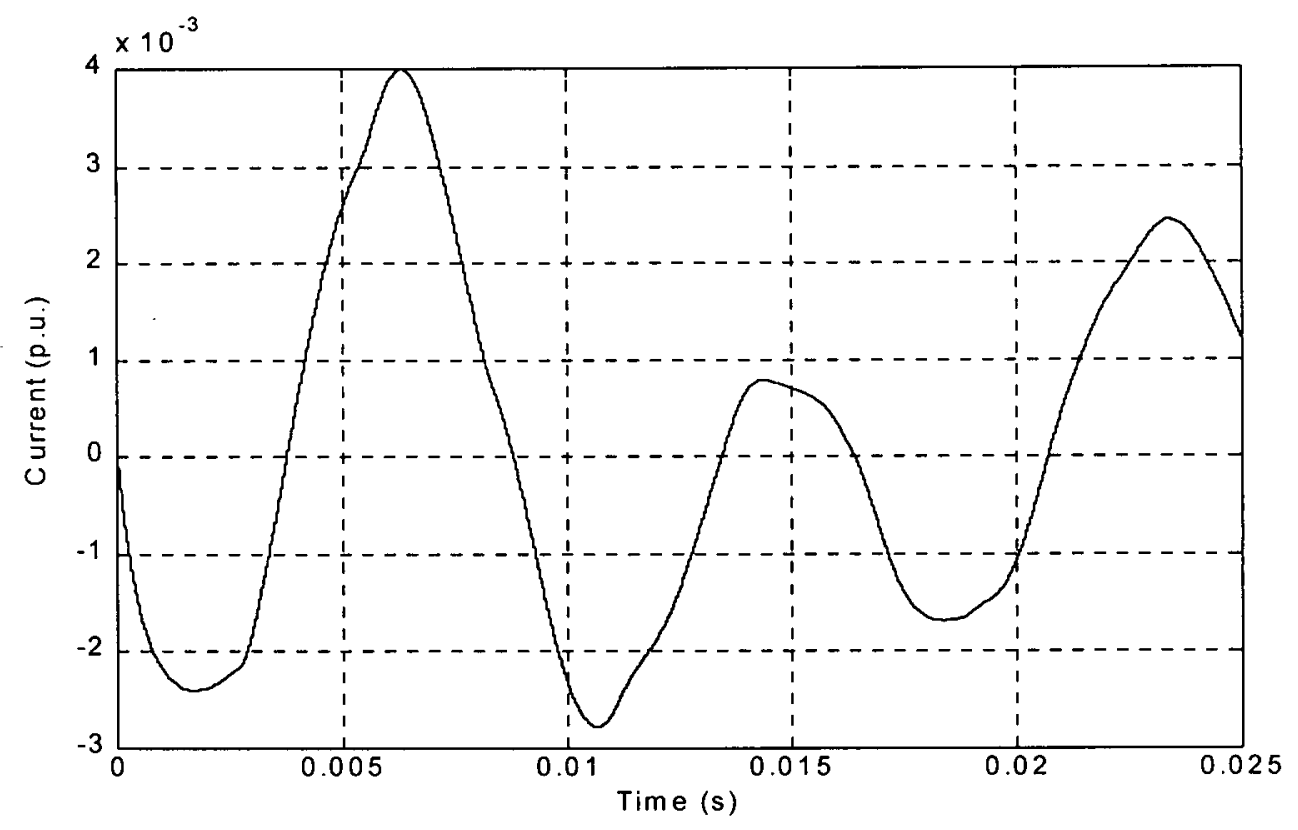

Figure 3.17 Zero sequence current and current in phase A

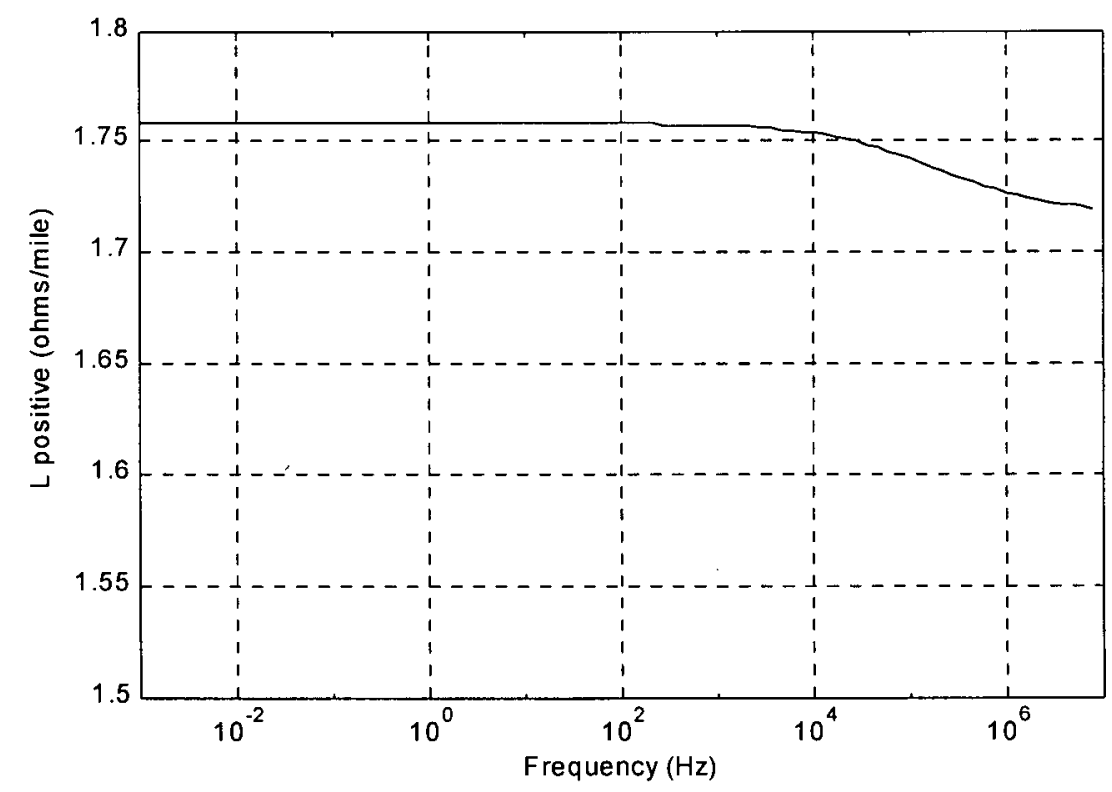

Figure 3.18 Positive sequence inductance of the three-phase line 


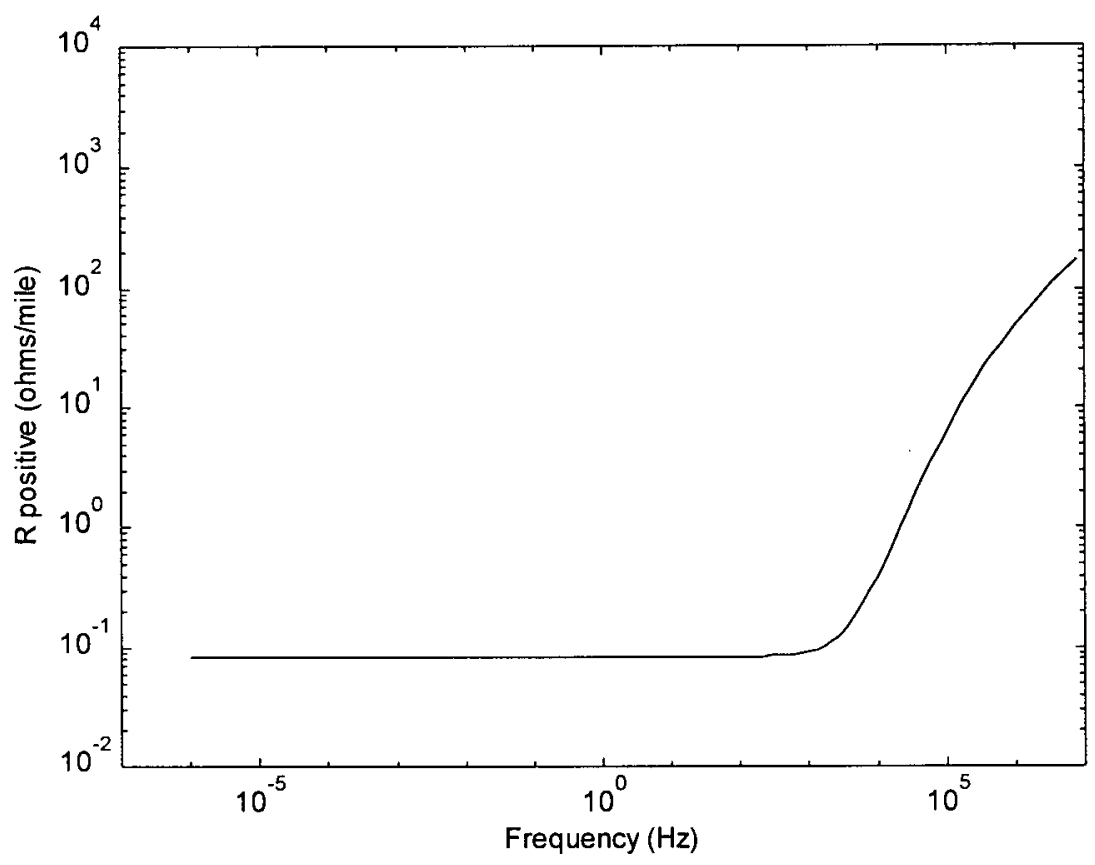

Figure 3.19 Positive sequence resistance of the three-phase line

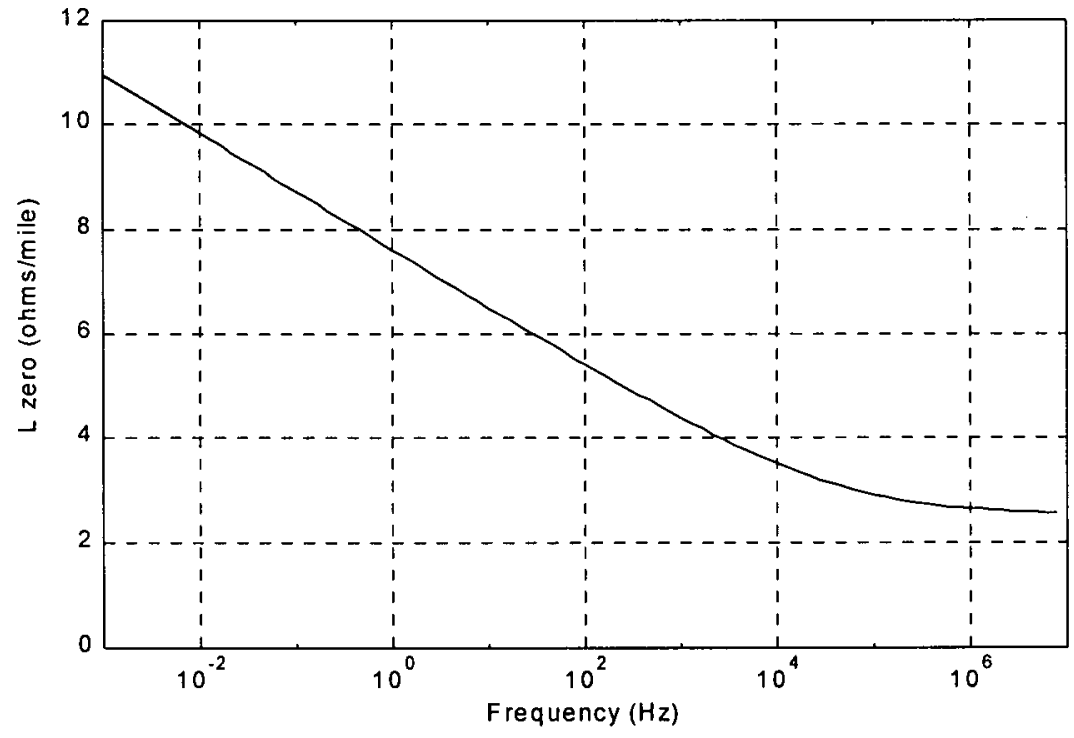

Figure 3.20 Zero sequence inductance of the three-phase line 


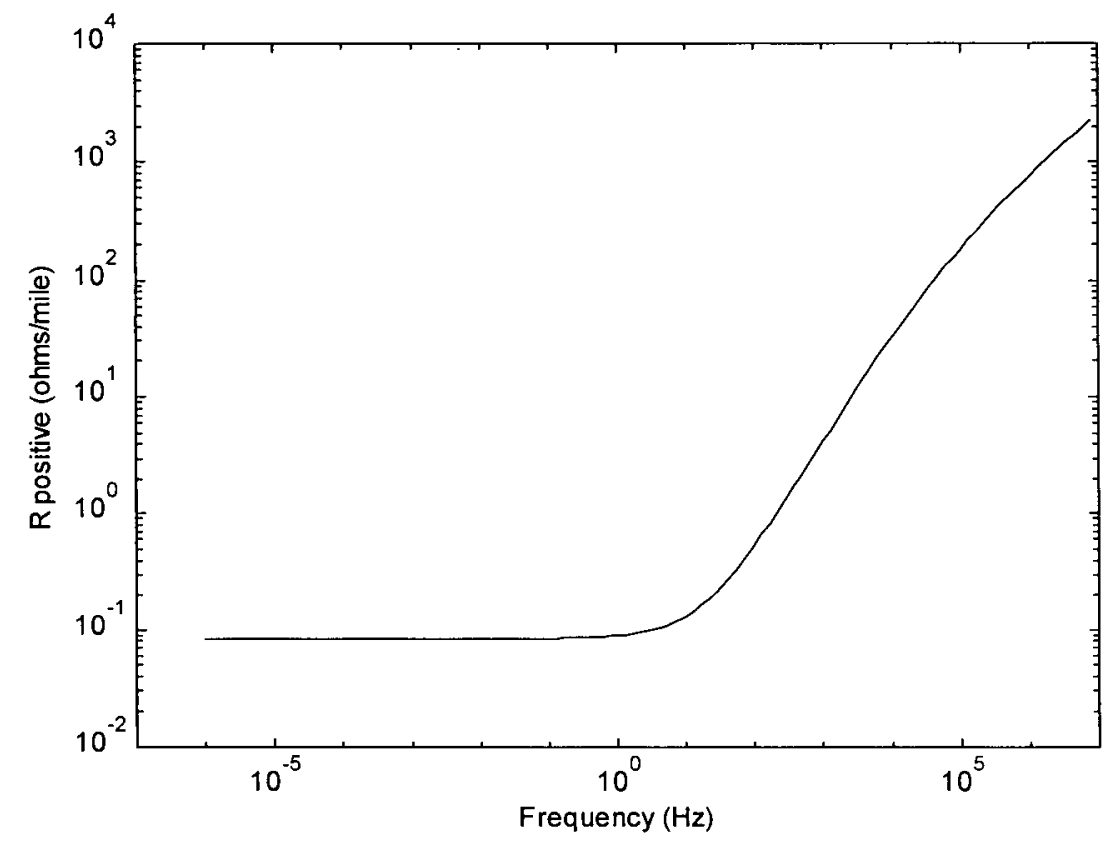

Figure 3.21 Zero sequence resistance of the tree-phase line

\section{The zero sequence current rules:}

"If the zero sequence current is small, then use the constant parameter line model".

"If the zero sequence current is high and contains high non-power frequencies, then the frequency dependent line model should be used". 


\subsubsection{Shunt Compensation}

Shunt reactors are usually modelled as a simple lumped inductance with a series resistance. A parallel resistance may be added for more realistic high frequency damping [14].

The main purpose of shunt compensation in EHV systems is to limit the power frequency overvoltages. Since the total overvoltage factors on closing and reclosing depend approximately linearly on the power frequency overvoltages, shunt compensation also has an important effect on the magnitude of the total overvoltages.

Table 3.2 shows the steady-state nodal voltages for the network of Figure 3.2 when shunt compensation is included at the sending end, while Table 3.3 shows the steady-state nodal voltages for the same network without shunt compensation. The total overvoltages will in general be higher for networks having little or no shunt compensation as compared with cases with more shunt compensation. Figure 3.22, Figure 3.23, and Figure 3.24 show the comparison between the overvoltages with and without shunt reactors at the receiving end for phase A, phase $B$ and phase $C$, respectively.

Table 3.4 shows the steady-state nodal voltages for the same network if the same shunt reactor at the sending end is also placed at the receiving end. As we can observe, the overvoltages are reduced even more than in the other two cases.

For $100 \%$ compensation, we need a shunt reactor at both ends of the line with a positive sequence value of $\frac{1}{X_{\text {pos }}}=\frac{1}{2} \omega C_{\text {pos }}^{\prime} l$. In this case, the voltages at both ends would be identi- 
cal under no-load condition as shown in Table 3.5. This can easily be shown with the nominal $\pi$-circuit of equation (1.1). In practice, $100 \%$ compensation is avoided because of the danger of resonance. Typically degrees of compensation are $50 \%$ to $70 \%$. In that case, $\frac{1}{X_{p o s}}=\frac{1}{2} \omega C_{p o s}^{\prime} l \times \frac{k}{100}$ where $k$ is the percentage of compensation.

\section{The shunt compensation rule:}

"If you want to have $k \%$ shunt compensation for the line, then place a shunt reactor with a positive sequence value of $\frac{1}{X_{\text {pos }}}=\frac{1}{2} \omega C_{\text {pos }}^{\prime} l \times \frac{k}{100}$ at both ends of the line".

Table 3.2:Network steady-state nodal voltages with shunt compensation

$\begin{array}{cc}\text { NAME } & \text { MAGNITUDE } \\ \text { JAG } & 0.8289 \\ \text { TAQ } & 0.9518\end{array}$

Table 3.3:Network steady-state nodal voltages without shunt compensation NAME MAGNITUDE $\mathrm{JAG} \quad 0.8770$

TAQ $\quad 1.007$


Table 3.4:Network steady-state nodal voltages with shunt compensation doubled

$\begin{array}{cc}\text { NAME } & \text { MAGNITUDE } \\ \text { JAG } & 0.8228 \\ \text { TAQ } & 0.9098\end{array}$

Table 3.5:Network steady-state nodal voltages with $100 \%$ shunt compensation NAME MAGNITUDE

$\begin{array}{ll}\text { JAG } & 0.710 \\ \text { TAQ } & 0.7120\end{array}$

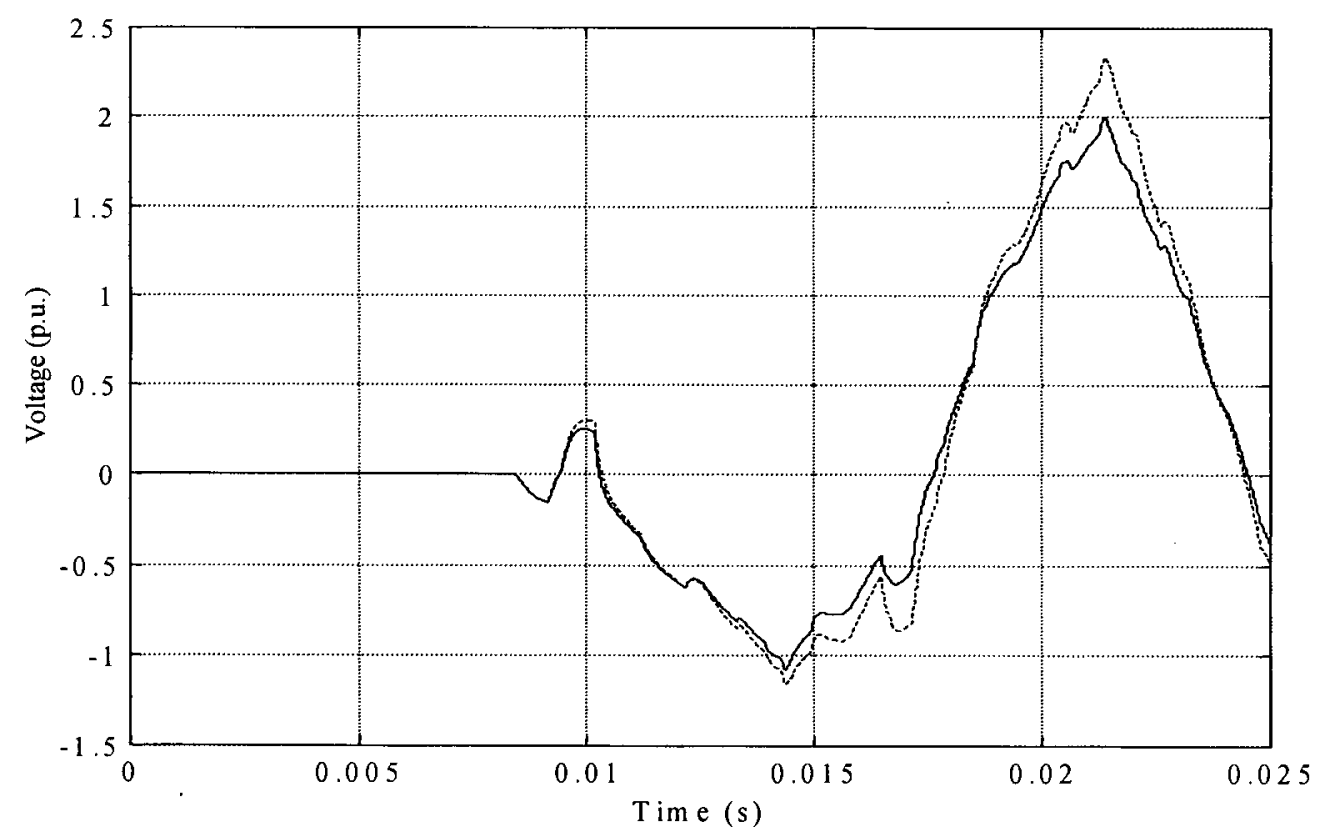

Figure 3.22 Overvoltages with shunt reactor (solid) and without shunt reactor (dashed) in phase A 


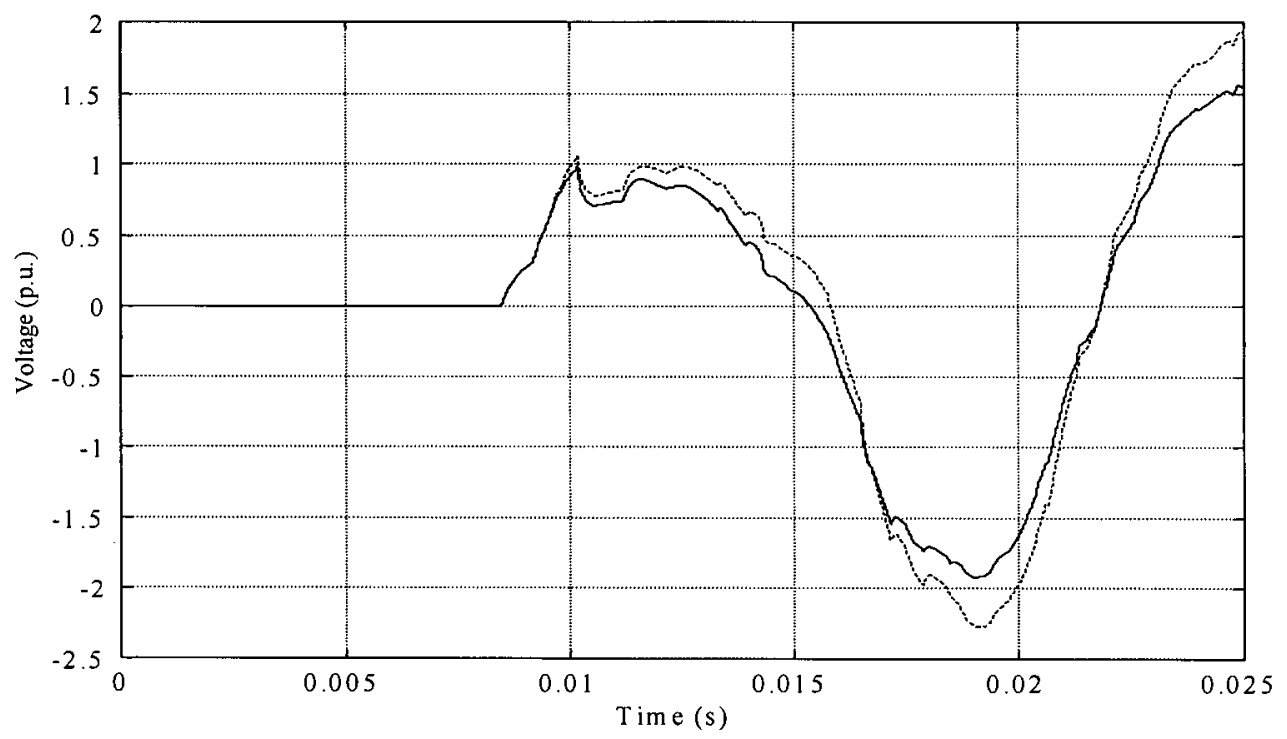

Figure 3.23 Overvoltages with shunt reactor (solid) and without shunt reactor (dashed) in phase B

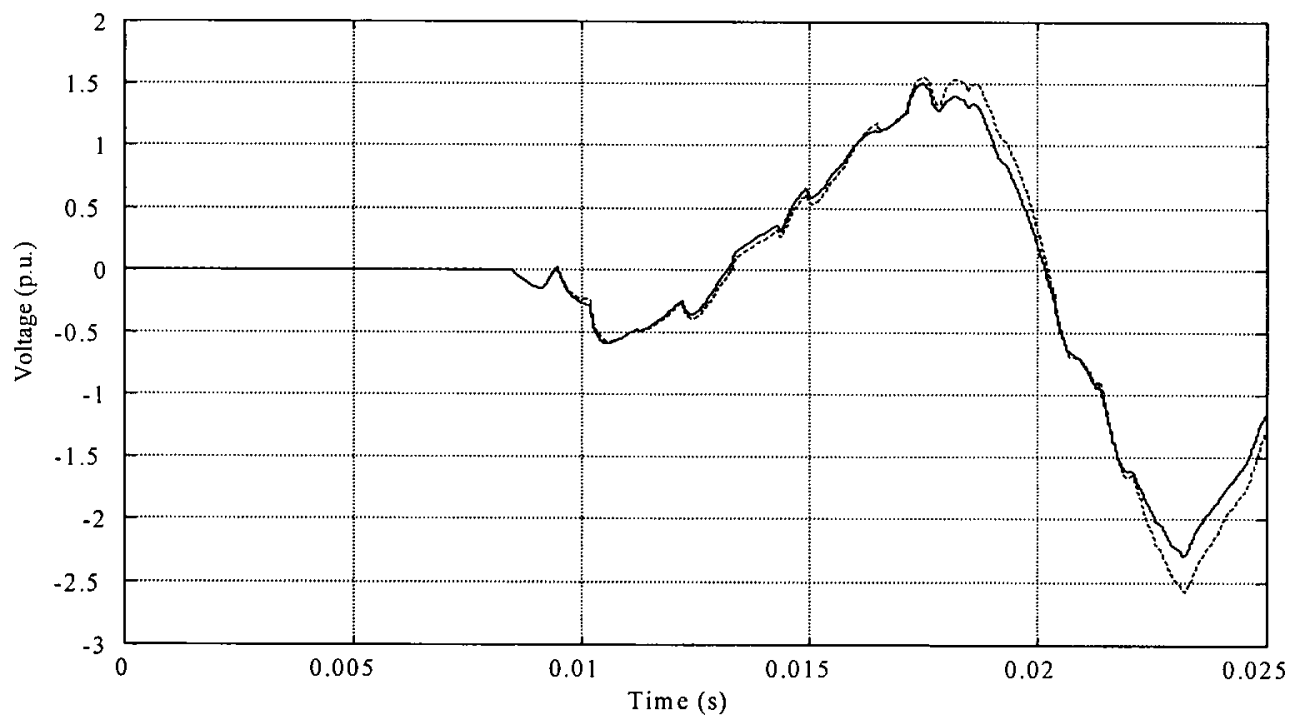

Figure 3.24 Overvoltages with shunt reactor (solid) and without shunt reactor (dashed) in phase C 


\subsubsection{Trapped Charges}

In switching surge studies, one must also simulate cases where it is assumed that the line to be energized has trapped charges on it, while the feeding network behind the circuit breaker will be in normal ac steady-state condition. This produces the highest overvoltages in the network. This applies to lines without shunt reactors only, because shunt reactors connected to the line (or inductive potential transformers) would drain off the trapped charges. The severity of the overvoltages in cases with trapped charges depends on the polarity of the trapped charges and the inserting instants of the breaker poles. There are two ways of simulating trapped charges in the EMTP:

1. Use the override "initial conditions" feature of the EMTP.

2. Let the circuit breaker opening action of switches trap a charge, before the circuit breakers are closed again.

To show the effect of trapped charges on switching surges, the following simulation was performed with trapped charges of $+1.0,-1.0,+0.5$ pu voltages on phases $A, B$, and $C$ respectively. The voltage waveforms for trapped charges are shown in Figure 3.25, Figure 3.26, and Figure 3.27.

\section{The trapped charges rule:}

"If you want to simulate trapped charges in the EMTP, then use the override "initial conditions" feature of the EMTP, or let the circuit breaker opening action of switches trap a charge before the circuit breakers are closed again". 


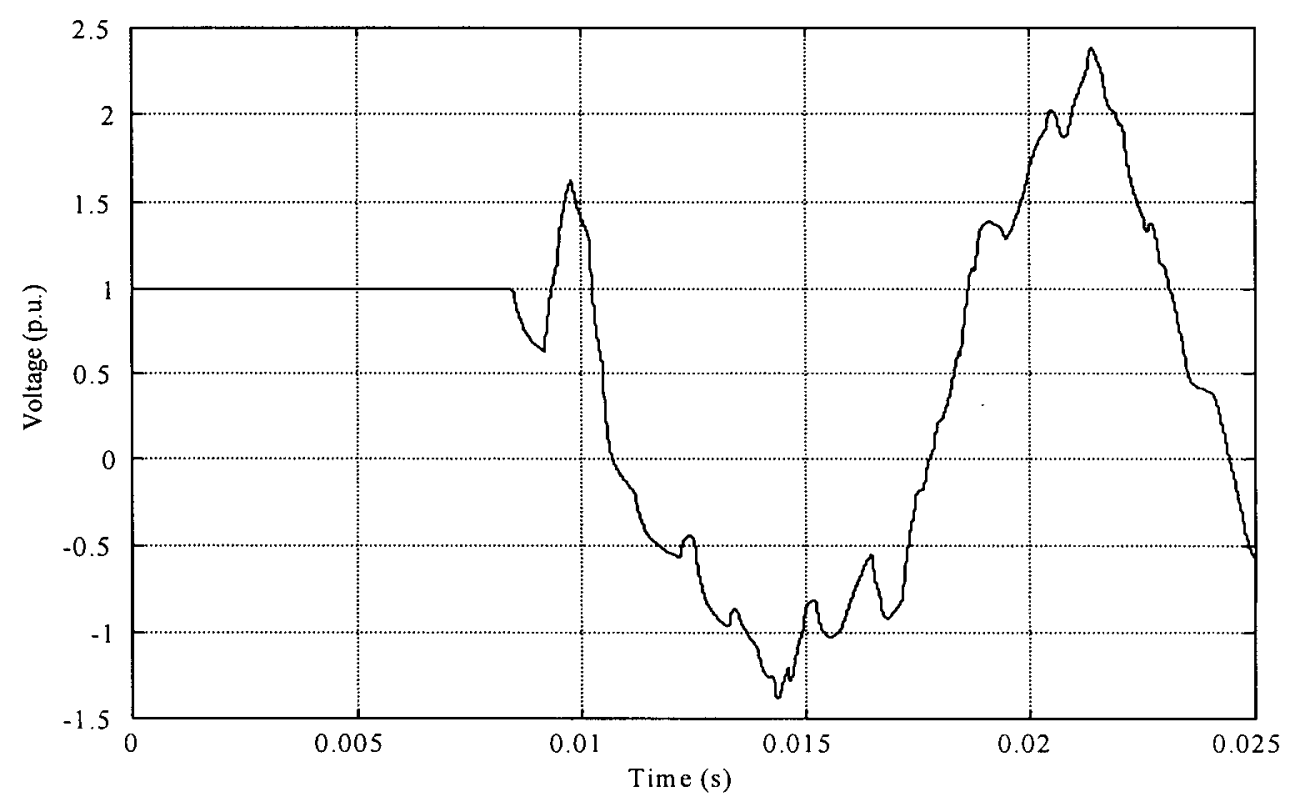

Figure 3.25 Effect of trapped charges on switching surge (1.0 p.u. on phase A)

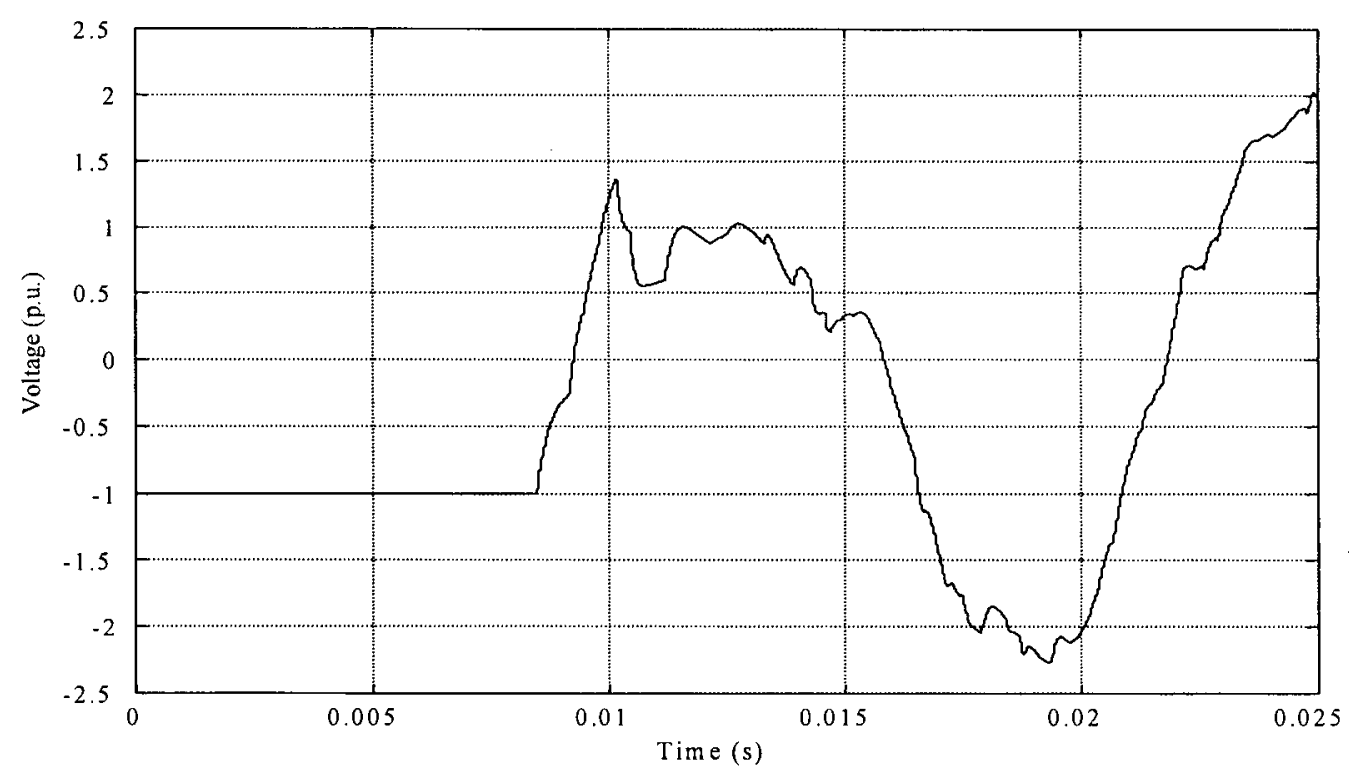

Figure 3.26 Effect of trapped charges on switching surge (-1.0 p.u. on phase B) 


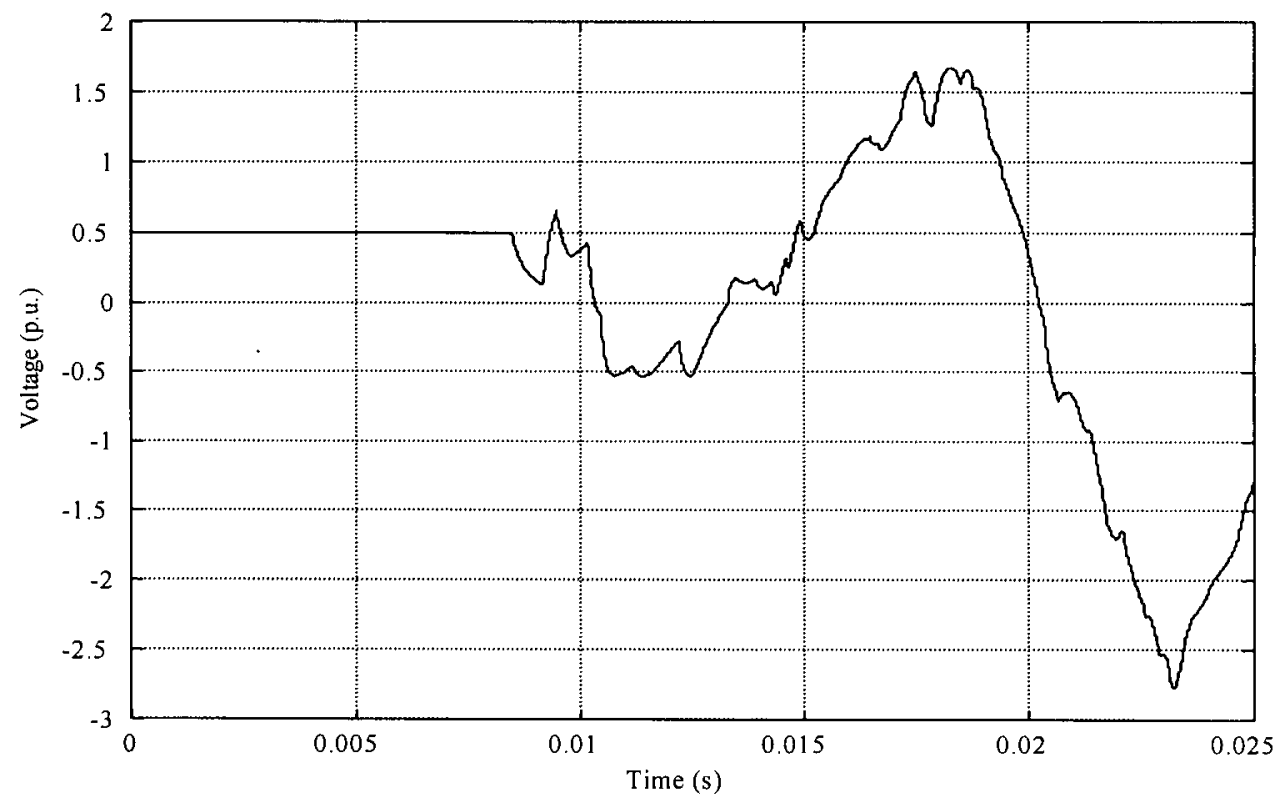

Figure 3.27 Effect of trapped charges on switching surge ( 0.5 p.u. on phase C)

\subsubsection{Feeding Network}

In most switching transient studies, the generators are modelled as voltage sources behind subtransient reactances, the non-switched lines with constant parameter models, and the transformers with their short-circuit impedances. How extensive the feeding network has to be modelled depends on the particular case. CIGRE Working Group 13.05 [3] recommends for normal switching operations "that the detailed model of the system in general must comprehend the part of network up to the second substations behind that of the operating circuit breaker. For line energization and re-energization exact representation only up to the first substations is sufficient in most cases". 
Often, a network equivalent which approximately represents the frequency response characteristic of the entire feeding network is used to simplify its representation [57][58][59].

In Figure 3.1 the distinction is made between inductive and complex source feeding networks. The results presented in [1] show that the overvoltages with complex feeding networks are typically $10 \%$ to $15 \%$ less than those with an inductive source, especially when no closing resistors are used.

\section{The feeding network rules:}

"If you want to model the feeding network for normal switching operations, then the detailed model of the system must include the part of the network up to the second substations behind that of the operating circuit breaker".

"If you want to model the feeding network for line energization and re-energization then line models only up to the first substations are sufficient in most cases".

"If the feeding network is complex, then the resulting overvoltages are typically 10\% to $15 \%$ less than those with an inductive source".

\subsubsection{Closing Resistors}

Circuit breakers are often equipped with closing resistors, which are inserted in series with the circuit for a short period of time before the main breaker contacts closed. Figure 3.1 shows that closing resistors are one of the most effective ways to reduce the switching overvoltages [1]. When shunt compensation is present, the effect is more assured. The selection of the opti- 
mum pre-insertion resistor value depends on the line shunt compensation, the short circuit power of the feeding network and the length of the line. For example, shorter lines have higher optimum pre-insertion resistors.

An example for the importance of closing resistors in reducing overvoltages is shown in Figure 3.28, Figure 3.29, and Figure 3.30 for phases A, B and C, respectively.

\section{The closing resistors rule:}

"If the circuit breaker has one-step closing resistors, then model each pole with two switches, one for the auxiliary contacts with the resistor and one for the main contact".

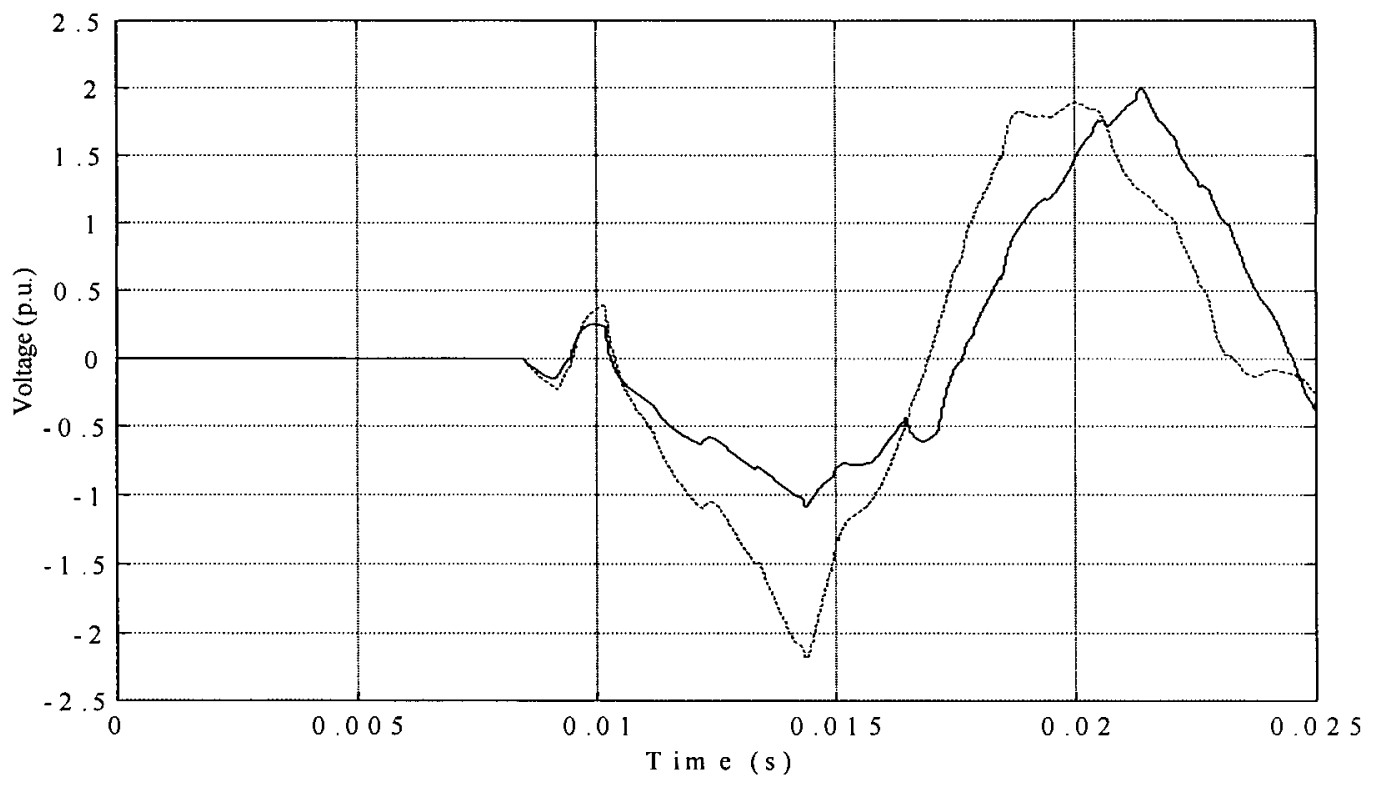

Figure 3.28 Overvoltages with closing resistors (solid) and without closing resistors (dashed) at phase $\mathrm{A}$ 


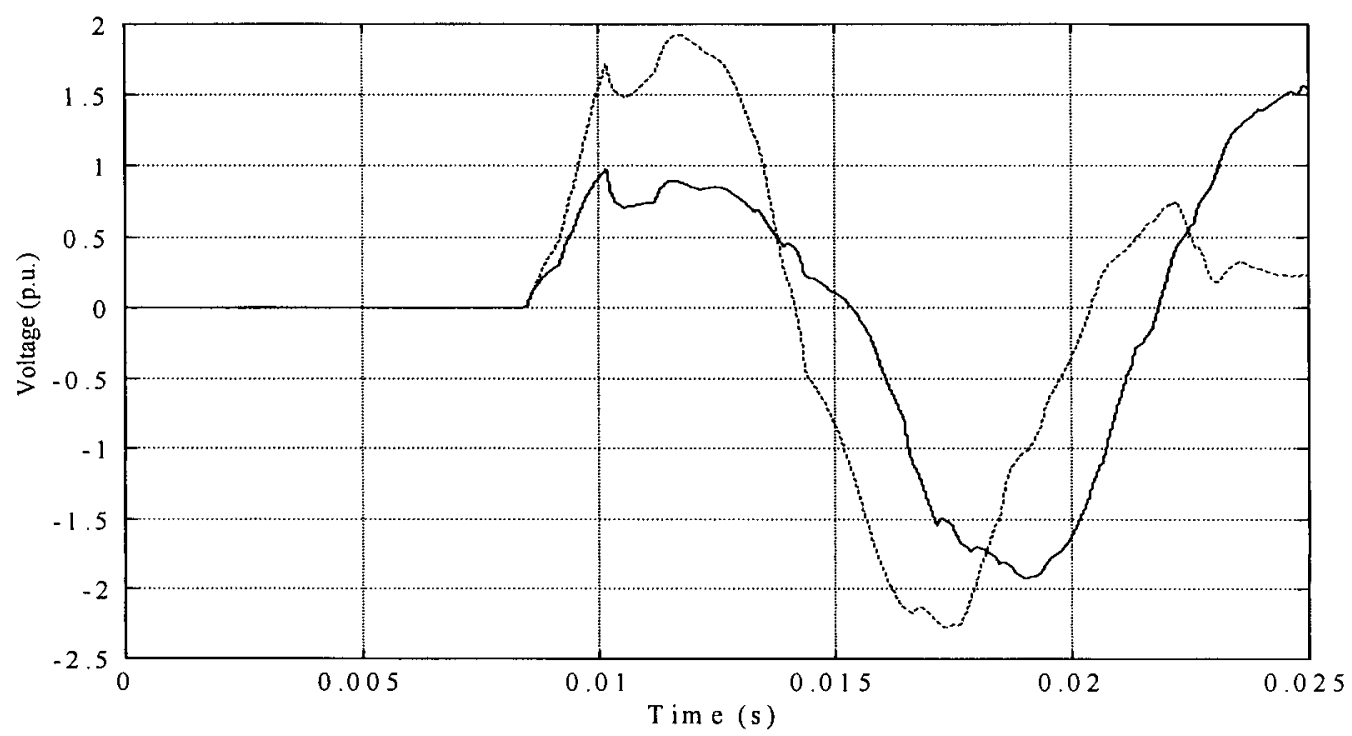

Figure 3.29 Overvoltages with closing resistors (solid) and without closing resistors (dashed) at phase $\mathrm{B}$

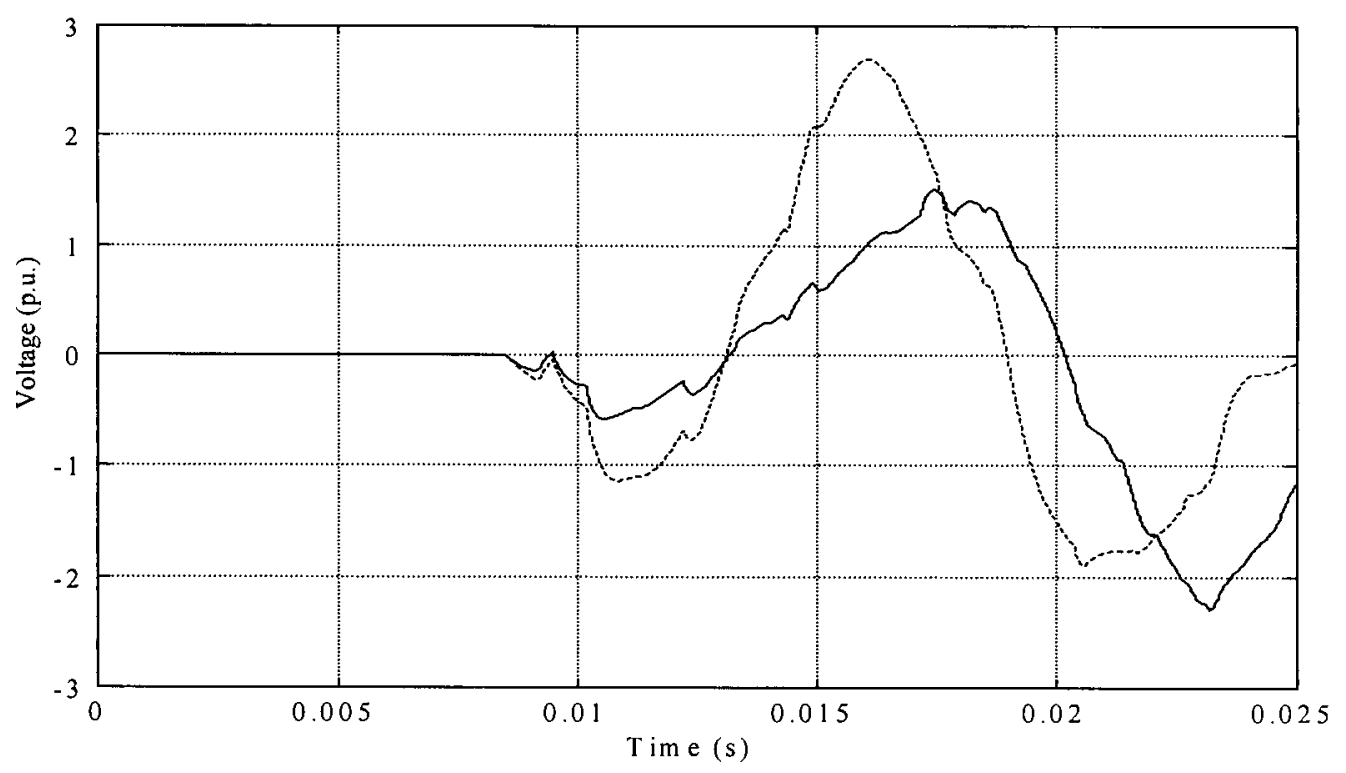

Figure 3.30 Overvoltages with closing resistors (solid) and without closing resistors (dashed) at phase $\mathrm{C}$ 


\subsubsection{Line Length}

Table 3.1 shows that the total overvoltages are strongly affected by the line length. The increase in the total overvoltages with the increasing line length is primarily due to the increase of the power frequency voltage, which is further a function of the shunt compensation and the short-circuit power of the feeding network. The transient overvoltage shows no clear dependence on the length of the switched line, but is influenced by closing resistors [1]. The given line energization case is simulated with a shorter line length of 125 miles and compared with the original simulation results. The effect of the line length is shown in Figure 3.31, Figure 3.32, and Figure 3.33 for phases A,B and C, respectively.

As we can observe, the overvoltages for the longer line are higher than those for the shorter one. This is because of the Ferranti rise effect phenomenon [15]. The rise in the receiving end voltage due to the Ferranti effect can be explained with a voltage divider equation, using the series impedance $R^{\prime} l+j \omega L^{\prime} l$ of the $\pi$-circuit and the shunt impedance $\frac{1}{\frac{1}{2} j \omega C^{\prime} l}$ at the receiving end of the $\pi$-circuit. Since $R^{\prime} « \omega L^{\prime}$ on high voltage lines, we can ignore the resistance and get:

$$
\frac{V_{2}}{V_{1}}=\frac{\frac{1}{\frac{1}{2} j \omega C^{\prime} l}}{\frac{1}{\frac{1}{2} j \omega C^{\prime} l}+j \omega L^{\prime} l}=\frac{1}{1-\frac{\omega^{2} L^{\prime} C^{\prime} l^{2}}{2}}
$$


Or

$$
\frac{V_{1}-V_{2}}{V_{2}}=\frac{\Delta V_{2}}{V_{2}}=-\frac{1}{2} \omega^{2} L^{\prime} C^{\prime} l^{2}
$$

This shows that the relative voltage drop $\frac{\Delta V_{2}}{V_{2}}$ is negative, which means a voltage rise, and that this relative voltage rise is proportional to the square of the line length.

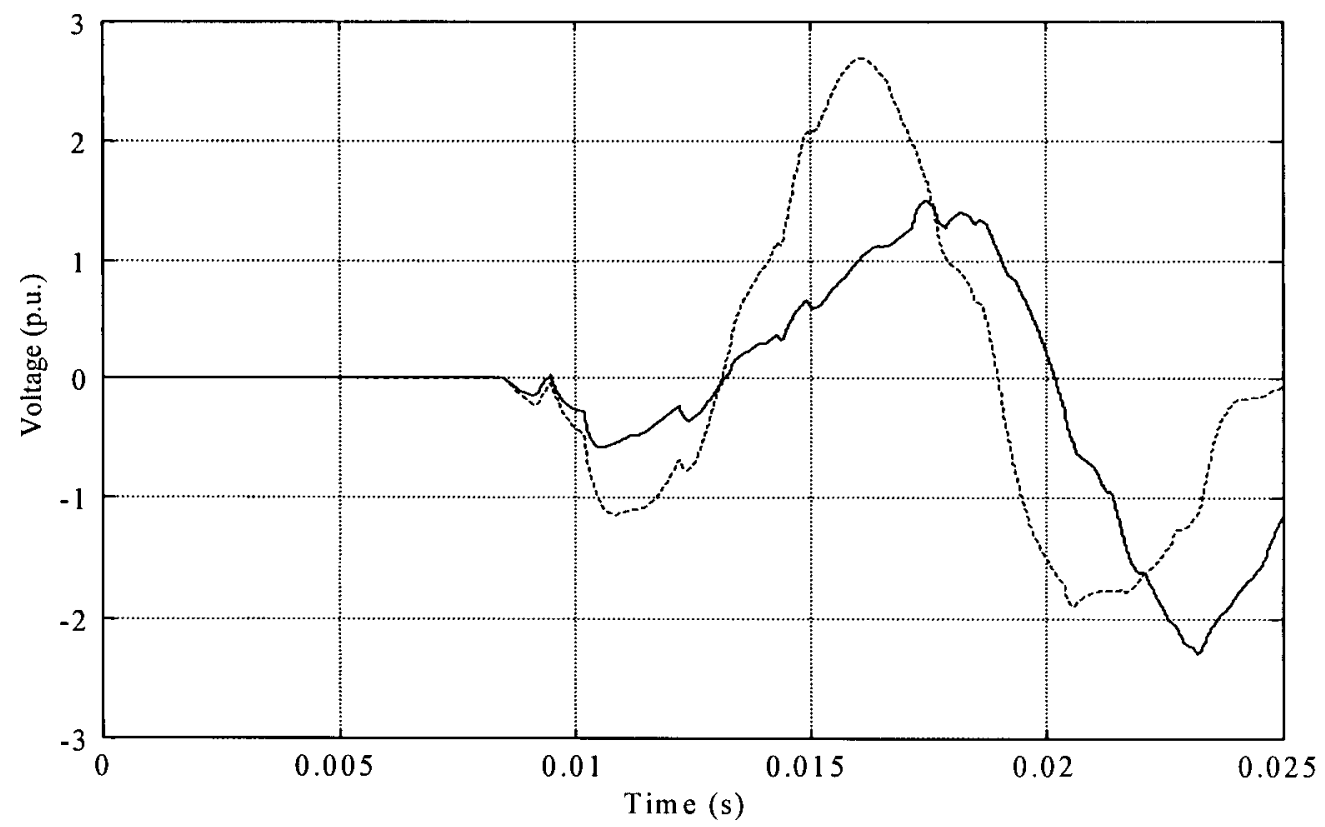

Figure 3.31 The effect of line length on the overvoltages in phase A: original line (solid), shorter line (dashed) 


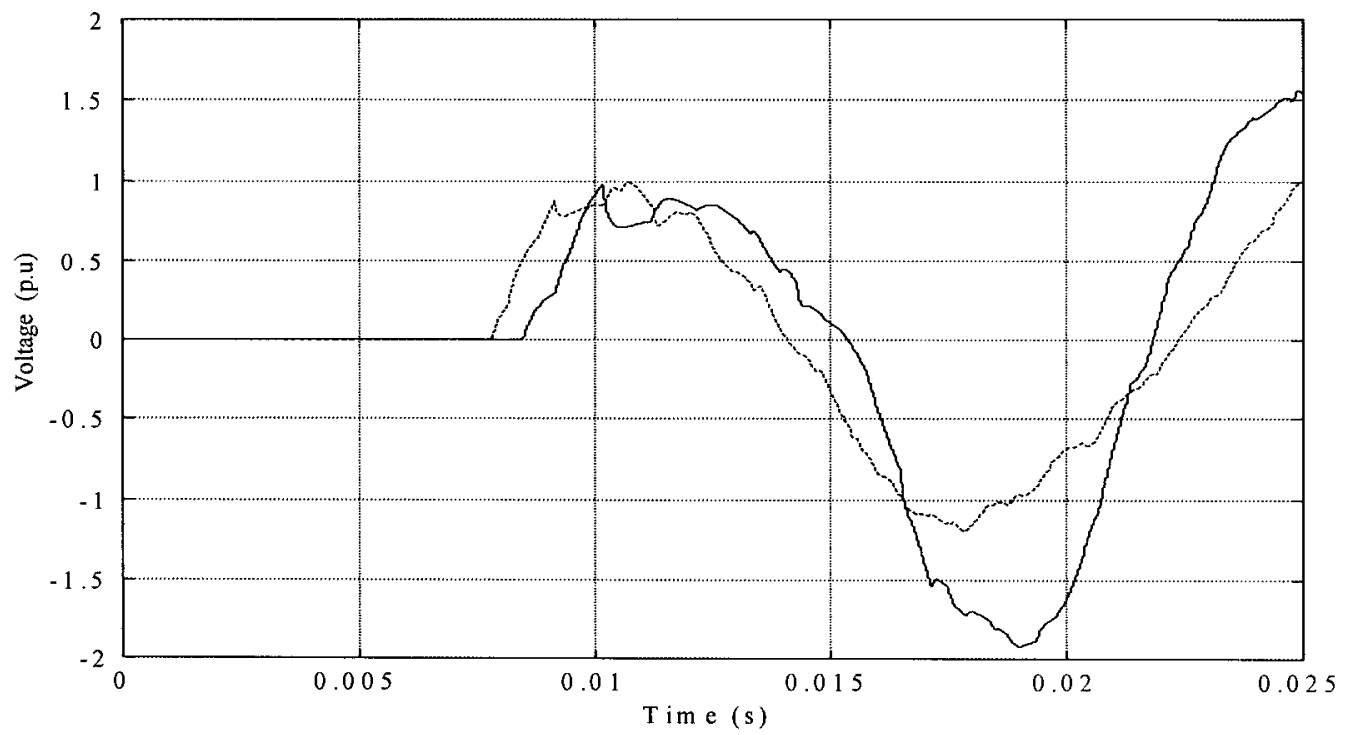

Figure 3.32 The effect of line length on the overvoltages in phase B: original line (solid), shorter line (dashed)

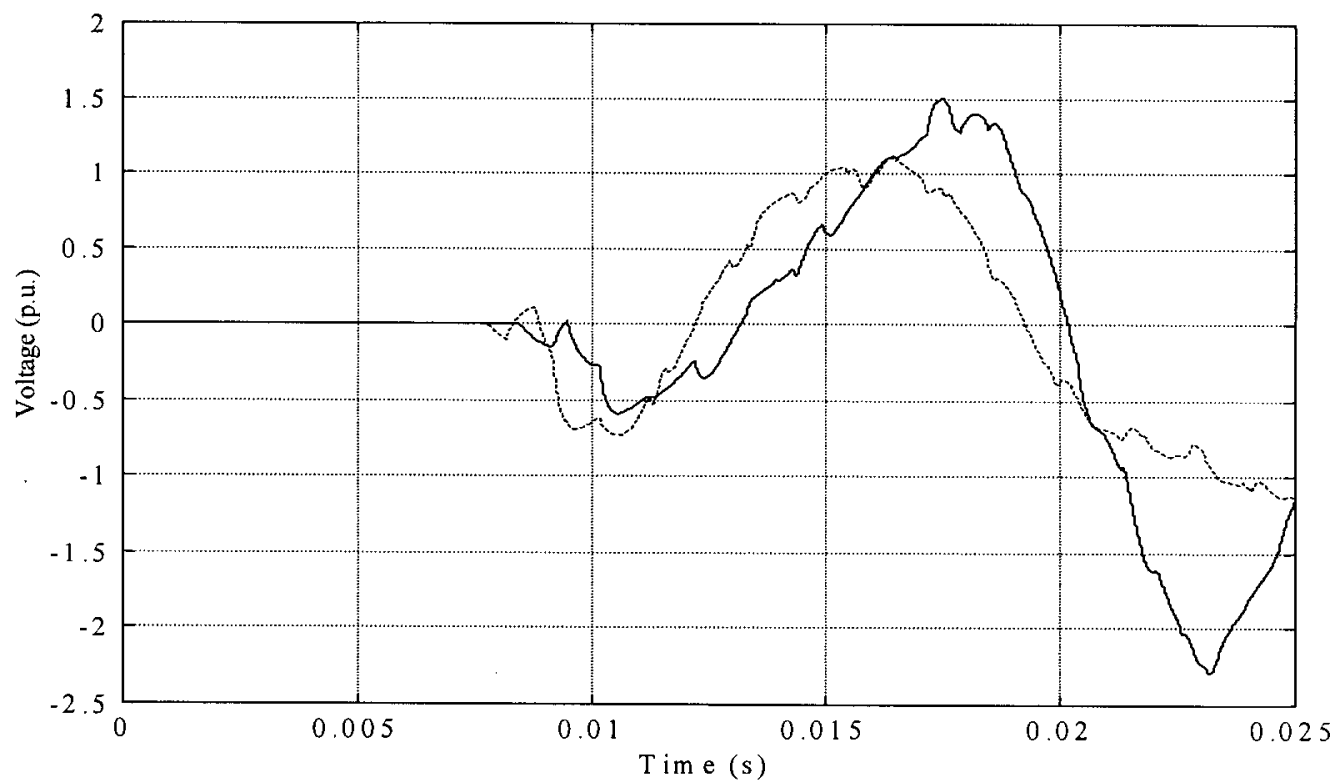

Figure 3.33 The effect of line length on the overvoltages in phase C: original line (solid), shorter line (dashed) 


\subsubsection{Closing Angle and Pole Span}

The closing angles of the three breaker poles are the phase angles of the source side voltages at the instant of electrical closure of the contacts. These angles have a strong influence on the line closing and reclosing overvoltages as they determine the initial conditions for the transients. For transients, when they are not controlled, undesired closing instants of the three poles may occur, but only within the limits of the breaker's pole span. The pole span is the time between the first and the last pole to close. When all the three breaker poles close simultaneously, the overvoltages are smaller than those of random closing. When closing resistors are used, the resistor insertion time should exceed the pole span of the breaker [1].

\subsubsection{Statistical Switching}

Transient voltage and current magnitudes depend upon the instant on the voltage waveform at which the circuit breaker contacts close electrically. A statistical switching case study typically consists of 100 or more separate simulations, each using a different set of circuit breaker closing times. Statistical methods can then be used to process the peak overvoltages from all the simulations.

The switching overvoltages that occur in any specific system arrangement follow a statistical distribution. The distribution function of the amplitude of the switching overvoltages does not behave according to a normal Gausssian distribution. There exists a minimum and a maximum overvoltage magnitude that cannot be exceeded due to technical reasons. 
The procedure for assessing the statistical distribution of overvoltages can be presented as follows:

1. Generate the input data file.

2. Run the case many times with different closing times to get a large enough random sample for the phase voltages.

3. Apply statistical methods to the phase voltages to get their statistical distribution.

4. Determine the protective level of the system considering both reliability and economy.

An extensive statistical analysis for the Jaguara-Taquaril case has been done in [16].

\section{Statistical switching rule:}

"To design the insulation of the line, run 100 cases or more, with statistical variation of the closing angles and pole spans. Normally, the $2 \%$ value on the cumulative frequency distribution curve is used to design overvoltages".

\subsubsection{Derived Practical Rules from the Knowledge Base}

From the knowledge base presented in the previous sections, several practical rules can be obtained. Some of these derived rules are presented in Table 3.6. These rules could be included in the proposed rule-based systems. 
Table 3.6: Some derived rules from the knowledge base for switching surge transients

\begin{tabular}{|c|c|}
\hline Parameters & Derived Rules \\
\hline \multirow{3}{*}{ Step Size } & $\begin{array}{l}\text { If the maximum frequency in your system is } f_{\max } \text {, then use the simulation step size } \\
\qquad \text { of } \frac{1}{10 \times f_{\max }}\end{array}$ \\
\hline & $\begin{array}{l}\text { If the step size } \Delta t \text { is larger than the travel time } \tau \text { of the shortest line in the net- } \\
\text { work, then the new line model should be used for lines with } \Delta t>\tau\end{array}$ \\
\hline & $\begin{array}{l}\text { If you want to check the accuracy of the simulation, then divide the used step size } \\
\qquad \text { by } 2 \text { and run the simulation again }\end{array}$ \\
\hline \multirow[b]{2}{*}{$\begin{array}{c}\text { Transmission Line } \\
\text { Models }\end{array}$} & If the zero sequence current is small, then use the constant parameter line model \\
\hline & $\begin{array}{c}\text { If the zero sequence current is high and contains high non-power frequencies, } \\
\text { then the frequency dependent line model should be used }\end{array}$ \\
\hline Shunt Compensation & $\begin{array}{l}\text { If you want to have } k \% \text { shunt compensation for the line, then place a shunt reac- } \\
\text { tor with a positive sequence value of } \frac{1}{X_{\text {pos }}}=\frac{1}{2} \omega C_{p o s}^{\prime} l \times \frac{k}{100} \text { at both ends of the } \\
\text { line }\end{array}$ \\
\hline Trapped Charges & $\begin{array}{l}\text { If you want to simulate trapped charges in the EMTP, then use the override "ini- } \\
\text { tial conditions" feature of the EMTP or let the circuit breaker opening action of } \\
\text { switches trap a charge before the circuit breakers are closed again }\end{array}$ \\
\hline \multirow{3}{*}{ Feeding Network } & $\begin{array}{l}\text { If you want to model the feeding network for normal switching operations, then } \\
\text { the detailed model of the system must include the part of the network up to the } \\
\text { second substations behind that of the operating circuit breaker }\end{array}$ \\
\hline & $\begin{array}{l}\text { If you want to model the feeding network for line energization and re-energiza- } \\
\text { tion then line models only up to the first substations are sufficient in most cases }\end{array}$ \\
\hline & $\begin{array}{l}\text { If the feeding network is complex, then the resulting overvoltages are typically } \\
10 \% \text { to } 15 \% \text { less than those with an inductive source }\end{array}$ \\
\hline Closing Resistors & $\begin{array}{l}\text { If the circuit breaker has one-step closing resistors, then model each pole with } \\
\text { two switches, one for the auxiliary contacts with the resistor and one for the } \\
\text { main contact }\end{array}$ \\
\hline Statistical Switching & $\begin{array}{l}\text { To design the insulation of the line, run } 100 \text { cases or more, with statistical varia- } \\
\text { tion of the closing angles and pole spans. Normally, the } 2 \% \text { value on the cumula- } \\
\text { tive frequency distribution curve is used to design overvoltages }\end{array}$ \\
\hline
\end{tabular}


Also, from the information presented in Figure 3.1 we can derive various rules for the results evaluation process. These rules can be formulated based on the average values, as well as the maximum and minimum values for the overvoltages. For example, if the overvoltages occur during reclosing without closing resistors, when the system consists of a complex feeding network and no shunt compensation of the transmission line is used, then the maximum overvoltage should not exceed 3.5 p.u. The same scenario can be repeated for different system conditions.

\subsection{Summary}

The following sections have been covered in this chapter: an overview of switching transient overvoltages, the parameters that influence the switching overvoltages, the means for limiting the switching overvoltages, a practical case study of transmission line energization, and modelling suggestions for the simulation of line energizations based on the parameters that influence the switching overvoltages.

Using the knowledge presented in this chapter as a starting point should increase the knowledge of EMTP users about transients phenomena, and how to apply this knowledge to the use of the EMTP for more complex studies. An understanding of power system transient phenomena is needed before sufficient expertise can be claimed; there is no other alternative for understanding the fundamentals of transient phenomena and what their effects are expected to be, and this is what this chapter is partially trying to answer. 
The next chapter presents a new transmission model for short lines and cables based on one of the rules that was suggested for a knowledge base for switching surges. This rule suggests that if the travel time is smaller than the step size then the new line model should be used to get proper results. 


\section{Chapter 4}

\section{Transmission Line Model}

\subsection{Introduction}

This chapter presents a new EMTP line model for the representation of short overhead transmission lines and cables [64][72][73]. This model overcomes the limitation of using a time step size not larger than the travel time. The interpolation errors inherent in this line model produce a filtering effect for higher frequencies.

For simulating short transmission lines and cables in electromagnetic transients programs of the EMTP type [5], nominal $\pi$-circuits are usually used when the travel time $\tau$ is less than the time step size $\Delta t$. This $\pi$-circuit approximation is used because the constant parameter line models [4] as well as the frequency dependent line models [61, 62] require that the step size should not be larger than the travel time. 
In this chapter, a new modelling approach is presented which overcomes the time step size constraint for short lines. This modelling approach was suggested in the previous chapter when the step size $\Delta t$ is larger that the travel time $\tau$, which is the case for short lines and cables.

The constant parameter line model will be used to explain the approach, and to keep the derivation simple.

The new line is tested for two case studies. The first one is a case of transmission line energization, with data taken from the Jaguara-Taquaril line tests discussed in the previous chapter [6][7][8]. Simulation results for this case show that the new line model gives results for a short line which are close to the constant parameter line model results, while results with a $\pi$ circuit show unrealistic high frequency oscillations.

The new transmission line model is also tested for the case of a drive system which involves power electronics devices [80]. In this case, the new line model is used to represent a short cable. The $\pi$-circuit and the constant parameter line models would give unrealistic voltage waveforms.

\subsection{Transmission Line Models-Background}

Transmission lines and underground cables are the main transmission links in power systems. Transmission lines usually extend over many kilometers and are affected by a wide variety of phenomena, from short circuits, to switching surges and to lightning discharges. To simulate 
these and other related phenomena in a power system simulation program, accurate transmission line models need to be developed. A number of transmission line and underground cables have been developed and successfully implemented in the EMTP. The Power Systems Research Group in the Department of Electrical and Computer Engineering at the University of British Columbia has developed a number of line models for electromagnetic studies in the past twenty years [60][61][62][66][67].

To introduce the proposed line model properly, the best known and widely used transmission line models in the EMTP are briefly reviewed. A comprehensive review of different transmission lines and their features is presented in [4] and [68].

\subsubsection{The $\pi$-Circuit}

The $\pi$-circuit is the simplest representation of a transmission line. Here, the line is represented with lumped elements: a series impedance and two shunt admittances for the complete line or for line segments if cascade connections of $\pi$-circuits are used. This model is also used by the Transient Network Analyzer. This model is a good choice for steady-state applications. However, the $\pi$-circuit is not the best choice for transient solutions because it cannot represent the frequency dependent parameters and one must also accept the unrealistic oscillations caused by the lumped parameters of the circuit even for short lines. The cascade connection of $\pi$-circuits may still be useful for untransposed lines. 


\subsubsection{The Constant Parameter Line Model}

The constant parameter line model was the first line model used in the EMTP [5]. In this line model, the capacitance and the inductance are evenly distributed along the line while the losses are lumped in three places along the line. This line model has a constraint that the step size must be smaller than the travel time.

\subsubsection{The Frequency Dependent Line Model}

The frequency dependent line model is one of the most successful models for considering the frequency dependence of the parameters of the transmission line in the EMTP. This model is very accurate for both single-phase lines and for multi-phase lines. This line model has the same step size constraint as the constant parameter line model.

The line model presented in this chapter overcomes the mentioned step size constraint of the constant parameter and the frequency dependent line models. This is important for short lines as well as for transient stability analysis.

\subsection{Transmission Line Model For Long Lines}

Before introducing the new line model, let us review the lossless line model that is implemented in the EMTP, based on Bergeron's method [9]. Figure 4.1 shows the equivalent circuit from which the model's most important property is immediately clear: Both terminals $\mathbf{k}$ and $\mathbf{m}$ are galvanically separated. The currents into the two terminals $\mathbf{k}$ and $\mathbf{m}$ are given by: 


$$
\left[\begin{array}{l}
i_{k}(t) \\
i_{m}(t)
\end{array}\right]=\left[\begin{array}{cc}
G_{c} & 0 \\
0 & G_{c}
\end{array}\right]\left[\begin{array}{l}
v_{k}(t) \\
v_{m}(t)
\end{array}\right]+\left[\begin{array}{l}
h_{k}(t) \\
h_{m}(t)
\end{array}\right]
$$

where the history vector is known from the currents and voltages of preceding time steps

$$
\left[\begin{array}{l}
h_{k}(t) \\
h_{m}(t)
\end{array}\right]=-\left[\begin{array}{cc}
0 & G_{c} \\
G_{c} & 0
\end{array}\right]\left[\begin{array}{c}
v_{k}(t-\tau) \\
v_{m}(t-\tau)
\end{array}\right]-\left[\begin{array}{ll}
0 & 1 \\
1 & 0
\end{array}\right]\left[\begin{array}{l}
i_{k}(t-\tau) \\
i_{m}(t-\tau)
\end{array}\right]
$$

and where $G_{c}$ is the reciprocal of the surge impedance $Z_{c}$ :

$$
G_{c}=\sqrt{C^{\prime} / L^{\prime}}
$$

Equation (4.2) shows that the conditions at one end depend on what happened at the other end at travel time $\tau$ earlier.

As long as the step size $\Delta \mathrm{t}$ is less than the travel time $\tau$, those conditions at the far end at time $(t-\tau)$, which appear at the near end at instant $t$, can be retrieved from "history" tables.

\subsection{The New Line Model For Short Lines}

The concept of this new line model was suggested in [63]. The model equations were derived and implemented in MicroTran (UBC-version of the EMTP) in co-operation with S. Henschel [64]. The new line model allows a smooth transition from electromagnetic transients phenomena to very slow dynamic phenomena, and vice versa. The use of the new line model for switching between electromagnetic transients and stability simulations with variable step size was described in [64] and [78]. The work here [72][73] concentrates on using the new line model for electromagnetic transient studies. 


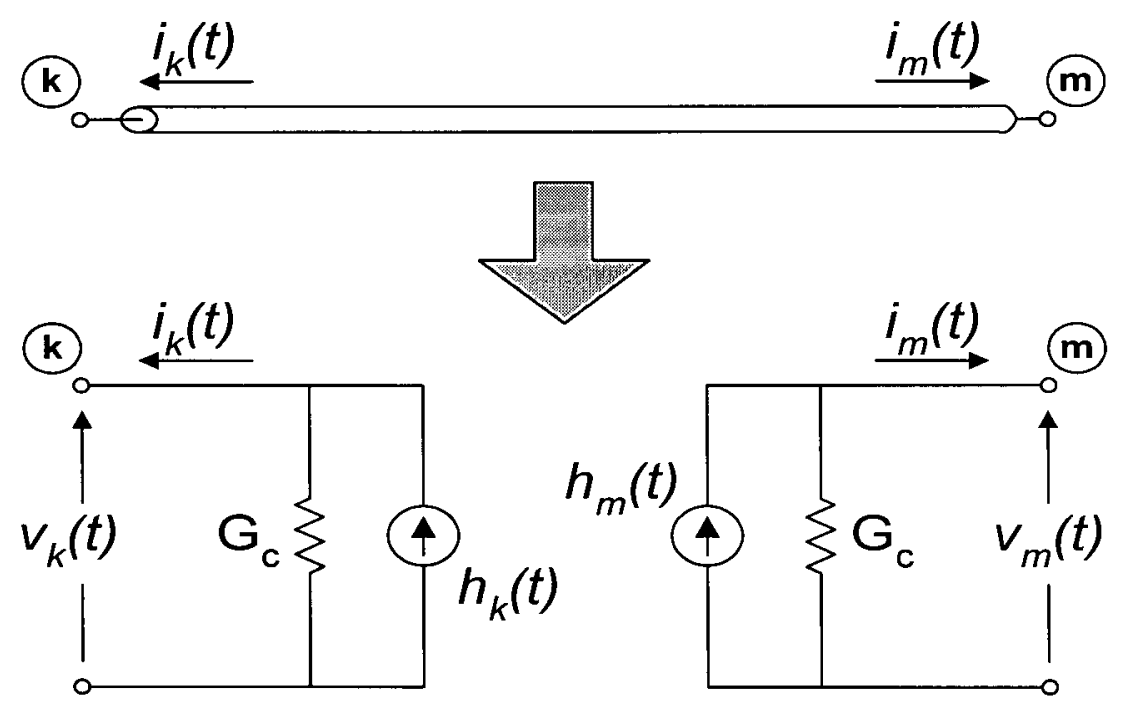

Figure 4.1 Lossless line model using Bergeron's method

The integration method used in the EMTP is the trapezoidal rule of integration. With this rule, we assume that a variable changes linearly as a straight line between two adjacent points. Therefore, if two solutions $x(t)$ and $x(t-\Delta t)$ were known, they could be linearly interpolated to yield $x(t-\tau)$ provided that $\Delta t \geq \tau$. The variable $x$ could either be a voltage or a current. These interpolated values can then be used to compute the history vector in equation (4.2).

The interpolation formula is:

$$
x(t-\tau)=a x(t)+b x(t-\Delta t)
$$

where

$$
a=\frac{\Delta t-\tau}{\Delta t} ; b=\frac{\tau}{\Delta t}
$$


By replacing the past values in equation (4.2) with the interpolated values, we can obtain a new model for the lossless line:

$$
\left[\begin{array}{l}
i_{k}(t) \\
i_{m}(t)
\end{array}\right]=\frac{G_{c}}{1-a^{2}}\left[\begin{array}{cc}
1+a^{2} & -2 a \\
-2 a & 1+a^{2}
\end{array}\right]\left[\begin{array}{l}
v_{k}(t) \\
v_{m}(t)
\end{array}\right]+\left[\begin{array}{l}
h_{k}(t) \\
h_{m}(t)
\end{array}\right]
$$

The history vector, whose values are obtained from the previous time step, is equal to:

$$
\begin{gathered}
{\left[\begin{array}{l}
h_{k}(t) \\
h_{m}(t)
\end{array}\right]=\frac{b G_{c}}{1-a^{2}}\left[\begin{array}{cc}
a & -1 \\
-1 & a
\end{array}\right]\left[\begin{array}{l}
v_{k}(t-\Delta t) \\
v_{m}(t-\Delta t)
\end{array}\right]} \\
+\frac{b}{1-a^{2}}\left[\begin{array}{cc}
a & -1 \\
-1 & a
\end{array}\right]\left[\begin{array}{l}
i_{k}(t-\Delta t) \\
i_{m}(t-\Delta t)
\end{array}\right]
\end{gathered}
$$

From equation (4.6) we can observe that the conductance matrix also possesses off-diagonal elements, so that the line equations can no longer be solved independently for both ends.

Equations (4.5) and (4.6) are only used if $\Delta t<\tau$; otherwise the EMTP automatically replaces them with equations (4.1) and (4.2).

The history vector of the new line model requires only values of the previous time step, so that the history memory of this model is extremely short.

The equivalent circuit of this new line model for the single-phase case is shown in Figure 4.2. 


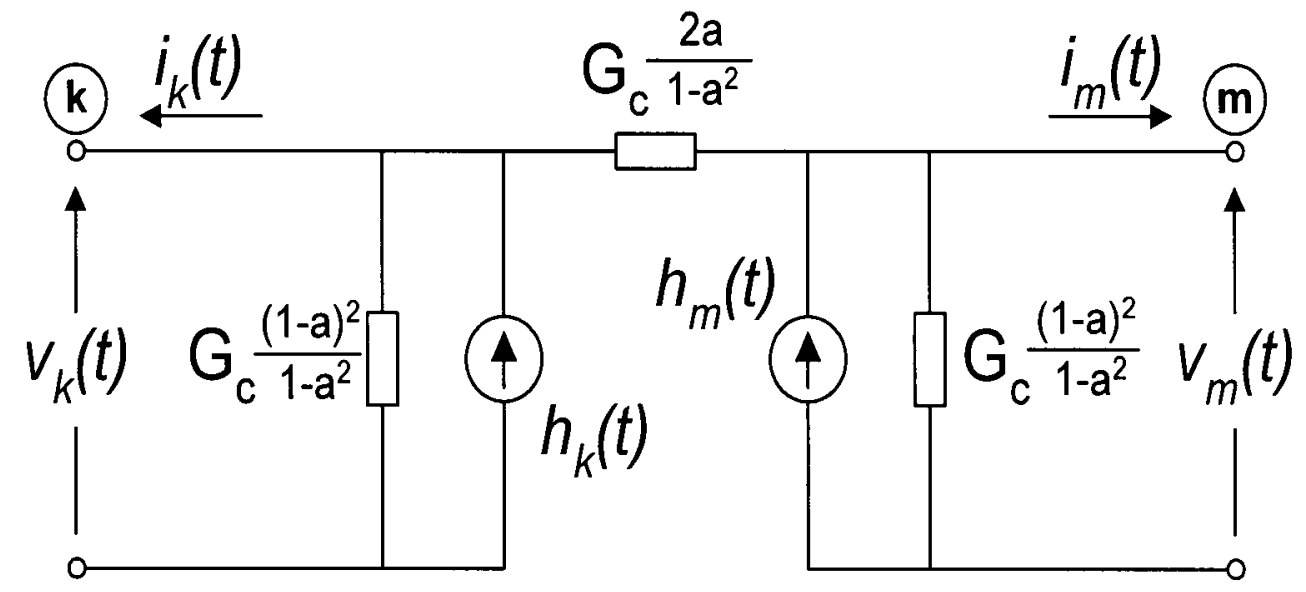

Figure 4.2 Lossless transmission line model

\subsection{Interpolation Error Analysis}

Interpolation with equation (4.4) produces errors which depend on frequency. It is therefore best to show them in the frequency domain. The errors of the new line model are derived here for the open and short-circuit responses, and the results are compared with those of the exact solution.

The new line model equations (4.5) and (4.6) can be transformed into the frequency domain as follows, with $I$ and $V$ being phasors:

$$
\left[\begin{array}{c}
I_{k} e^{j \omega t} \\
I_{m} e^{j \omega t}
\end{array}\right]=\frac{G_{c}}{1-a^{2}}\left[\begin{array}{cc}
1+a^{2} & -2 a \\
-2 a & 1+a^{2}
\end{array}\right]\left[\begin{array}{l}
V_{k} e^{j \omega t} \\
V_{m} e^{j \omega t}
\end{array}\right]+\left[\begin{array}{l}
H_{k} e^{j \omega t} \\
H_{m} e^{j \omega t}
\end{array}\right]
$$

where: 


$$
\begin{gathered}
{\left[\begin{array}{c}
H_{k} e^{j \omega t} \\
H_{m} e^{j \omega t}
\end{array}\right]=\frac{b G_{c}}{1-a^{2}}\left[\begin{array}{cc}
a & -1 \\
-1 & a
\end{array}\right]\left[\begin{array}{c}
V_{k} e^{j \omega(t-\Delta t)} \\
V_{m} e^{j \omega(t-\Delta t)}
\end{array}\right]} \\
+\frac{b}{1-a^{2}}\left[\begin{array}{cc}
a & -1 \\
-1 & a
\end{array}\right]\left[\begin{array}{l}
I_{k} e^{j \omega(t-\Delta t)} \\
I_{m} e^{j \omega(t-\Delta t)}
\end{array}\right]
\end{gathered}
$$

After dividing equations (4.7) and (4.8) by $e^{j \omega t}$, we obtain the phasor equation:

$$
\left[\begin{array}{c}
I_{k} \\
I_{m}
\end{array}\right]=\left[\begin{array}{cc}
Y_{k k} & Y_{k m} \\
Y_{m k} & Y_{m m}
\end{array}\right]\left[\begin{array}{c}
V_{k} \\
V_{m}
\end{array}\right]
$$

where:

$$
\begin{gathered}
Y_{k k}=Y_{m m}=\frac{-G_{c}\left(a^{2}+2 a b e^{-j \omega \Delta t}+b^{2} e^{-2 j \omega \Delta t}+1\right)}{\left(a^{2}+2 a b e^{-j \omega \Delta t}+b^{2} e^{-2 j \omega \Delta t}-1\right)} \\
Y_{k m}=Y_{m k}=\frac{2 G_{c}\left(a+b e^{-j \omega \Delta t}\right)}{\left(a^{2}+2 a b e^{-j \omega \Delta t}+b^{2} e^{-2 j \omega \Delta t}-1\right)}
\end{gathered}
$$

The exact solution for the lossless line model with constant parameter can be obtained from:

$$
\left[\begin{array}{c}
I_{k} \\
I_{m}
\end{array}\right]=G_{c}\left[\begin{array}{ll}
\frac{\cos \omega \tau}{j \sin \omega \tau} & \frac{-1}{j \sin \omega \tau} \\
\frac{-1}{j \sin \omega \tau} & \frac{\cos \omega \tau}{j \sin \omega \tau}
\end{array}\right]\left[\begin{array}{c}
V_{k} \\
V_{m}
\end{array}\right]
$$

If the receiving end is short circuited, the receiving end voltage equals zero $\left(V_{m}=0\right)$ and the short-circuit current ratio is given as: 


$$
\frac{I_{m}}{I_{k}}=\frac{Y_{k k}}{Y_{m k}}
$$

On the other hand, if the receiving end is open-circuited, the receiving end current equals zero $\left(I_{m}=0\right)$ and the open-circuit voltage ratio is given as:

$$
\frac{V_{m}}{V_{k}}=-\frac{Y_{m k}}{Y_{m m}}
$$

Equations (4.13) and (4.14) represent the short and open-circuit responses of the transmission line model at any given frequency $\omega$.

Figure 4.3 compares the magnitude of the short-circuit response for the exact frequency response (solid line), with that of the new line model (dashed lines from bottom to top), for step sizes $\Delta t$ equal $1.3 \tau, 1.6 \tau, 1.9 \tau$ and $2.2 \tau$, respectively. The values were obtained for $Z=$ $100 \Omega$ and $\tau=50 \mu \mathrm{s}$.

Figure 4.4 compares the magnitude of the open-circuit response for the exact model (solid line), with that of the new line model (dashed lines from bottom to top), for the same step sizes.

From the results shown in Figure 4.3 and Figure 4.4, it can be seen that the short and opencircuit responses of the new line model show a strong filtering effect, which becomes more emphasized as the simulation step size is increased. In a time domain simulation with $\Delta t \geq \tau$, this effect results in averaging of the transient voltage and current oscillations. The results with this filtering effect may come closer to reality. To prove this, comparisons with 
frequency dependent line models should be done in future research.

The results obtained in the frequency domain can also be duplicated in the time domain at a given frequency using the EMTP. The comparison between the time domain simulation and the frequency domain results shows that both answers are practically identical. The EMTP data file for the new line model is shown in Table A.5 in the Appendix D. The time domain simulation for the currents $I_{m}$ and $I_{k}$ at this point $(\Delta t=1.3 \tau$ and $f=1000 \mathrm{~Hz})$ are shown in Figure 4.5 and Figure 4.6. From these two figures, we can calculate the short-circuit response for the given point using equation (4.13). The results obtained are practically equal to the short circuit response that is measured in the frequency domain as $\frac{I_{m}}{I_{k}} \approx 0.98$.

The interpolation error analysis for the new line model, as compared with other line models and the exact solution, was implemented in MATLAB environment and it is presented in Appendix C. 


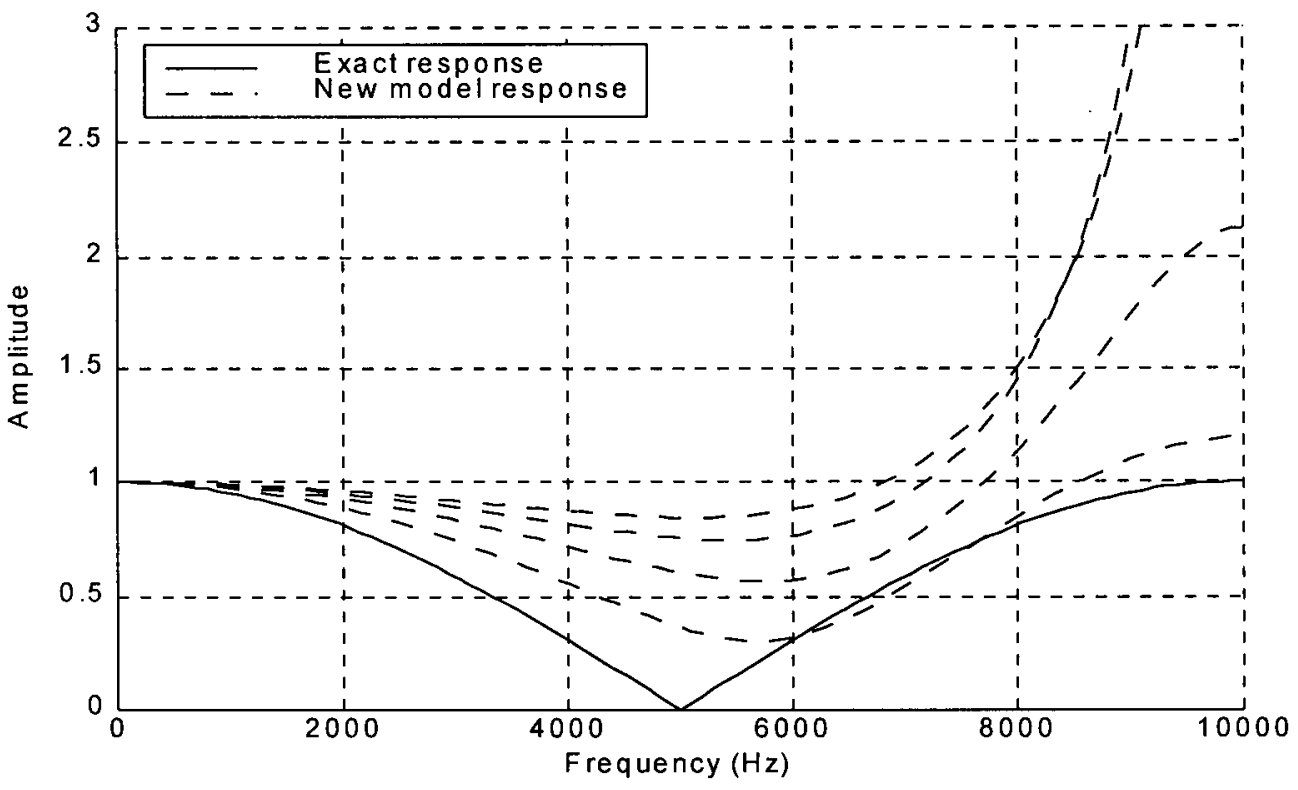

Figure 4.3 The short-circuit ratio

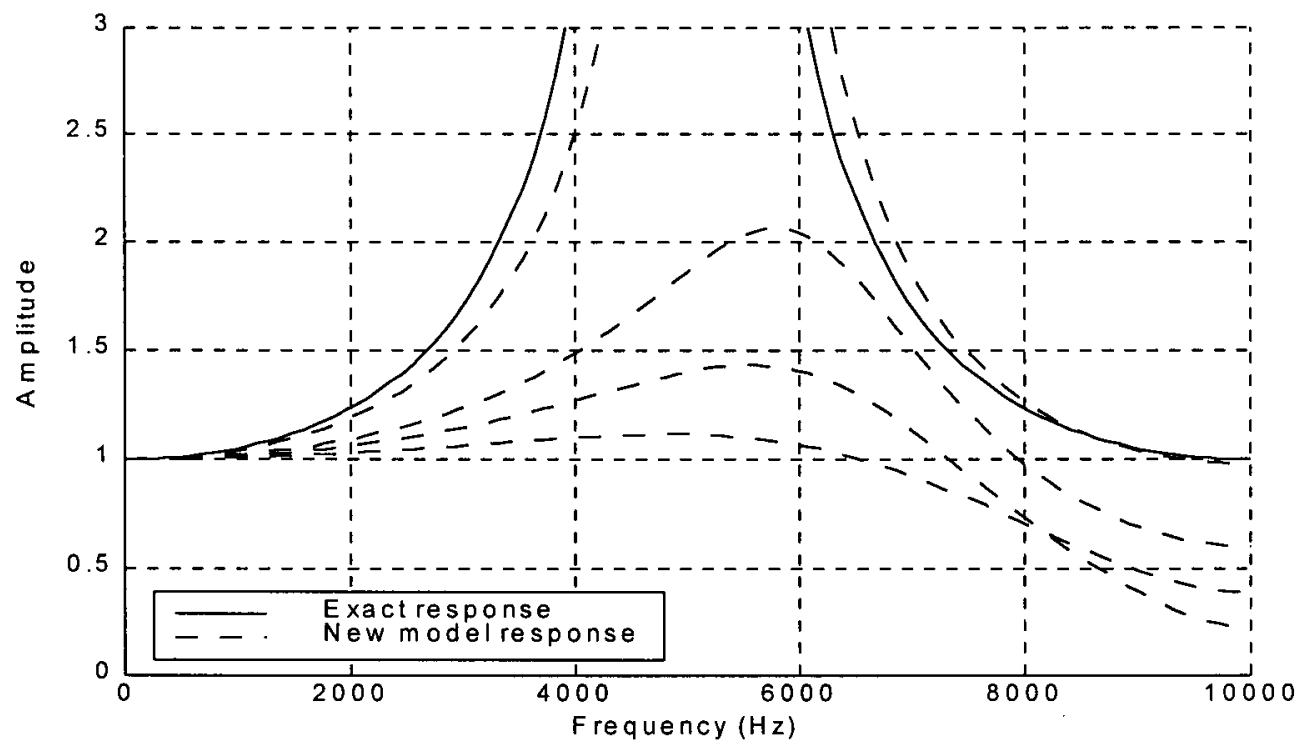

Figure 4.4 The open-circuit ratio 


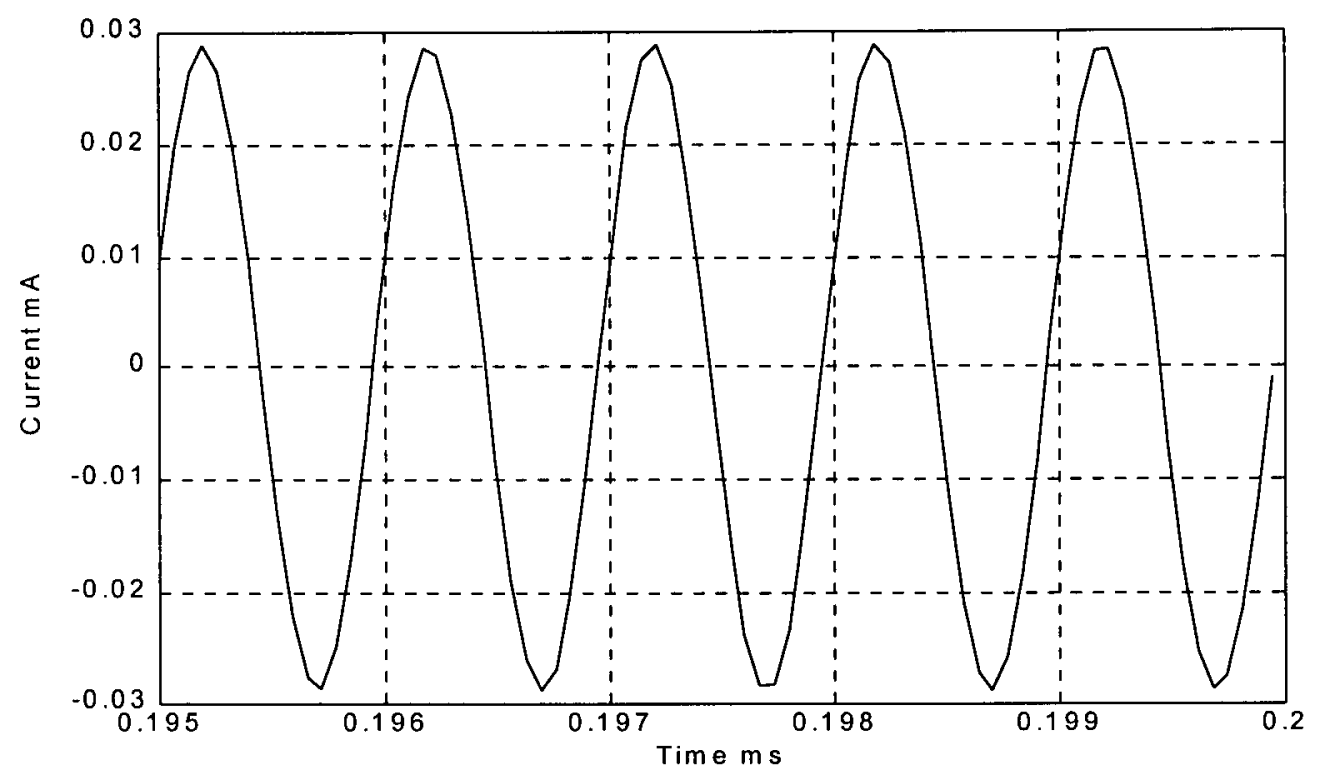

Figure 4.5 The Current $I_{m}$ response in the time domain

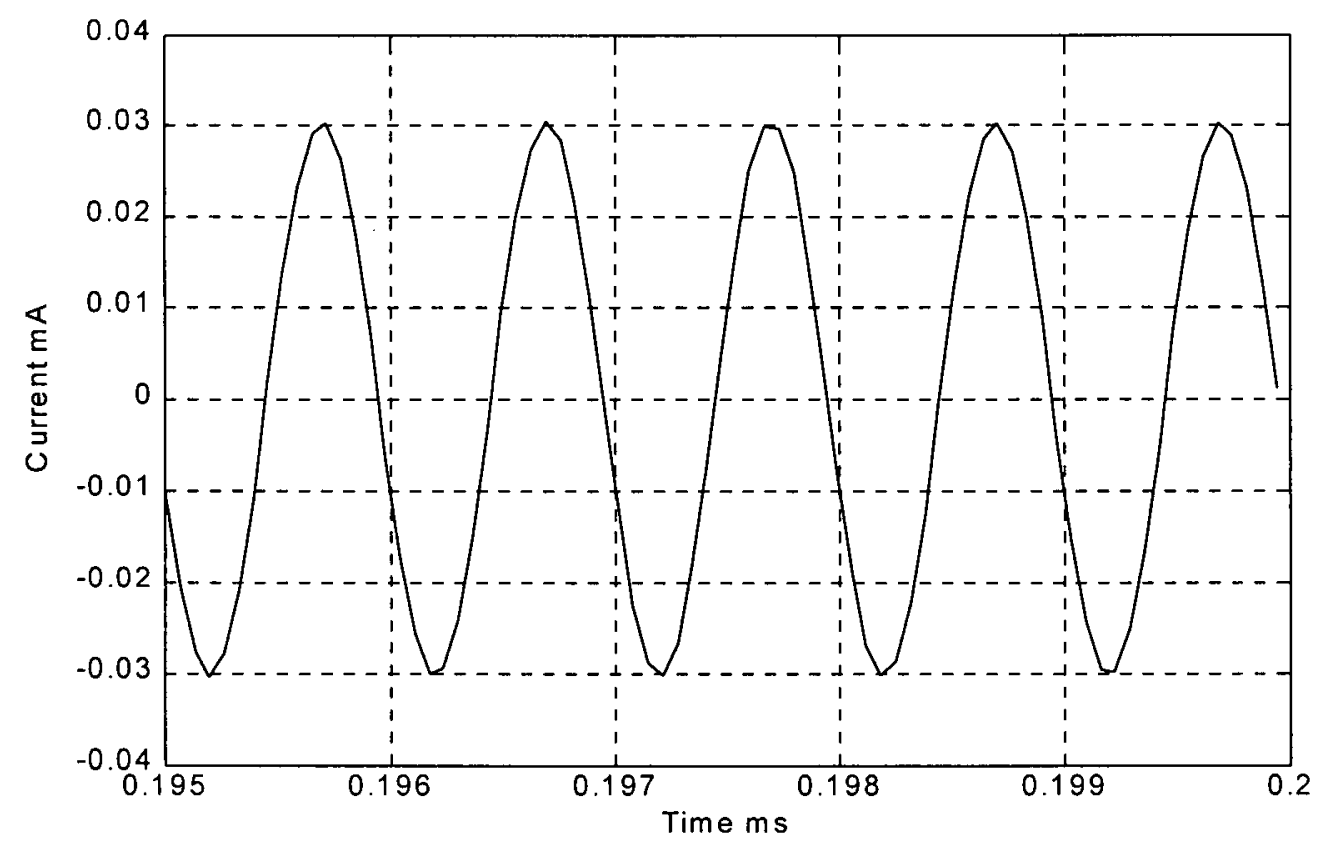

Figure 4.6 The current $I_{k}$ response in the time domain 


\subsection{The Lossy Line Model}

To represent the losses approximately, lumped resistances are inserted at both ends and in the middle of two lossless line sections, as shown in Figure 5. This approach is also used for the polyphase case [64]. The lossy line model was implemented in MicroTran (UBC-version of the EMTP). The connec subroutine for this implementation is presented in Appendix B.

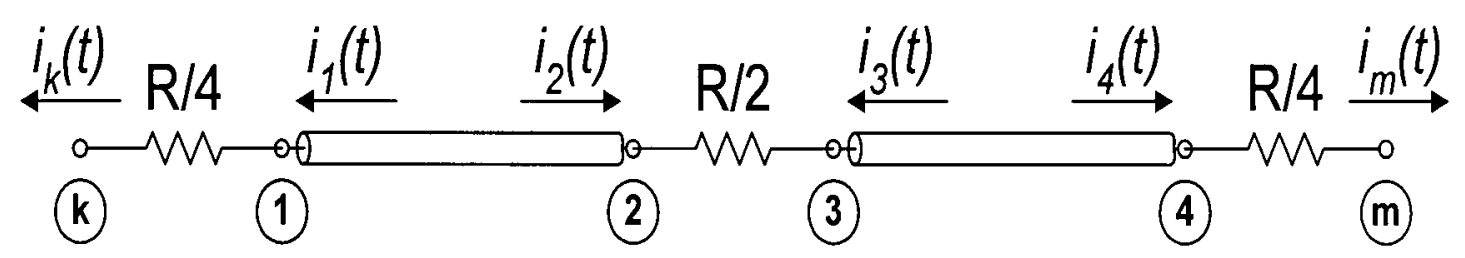

Figure 4.7 Schematic of a lossy line model

\subsection{Case Studies}

In order to demonstrate the usefulness of the proposed line model, a time domain simulation of transmission line energization and adjustable speed drive cases are used. The results and analyses are presented in this section.

\subsubsection{Transmission Line Energization}

A transmission line energization case is shown in Figure 4.8. The data comes from the Jaguara-Taquaril tests conducted by a Brazilian utility company [6][7][8].

To show the performance of the new line model as compared with other EMTP line models, the transmission line is divided into two sections. The longer section is represented by a 
constant parameter line model, while the short section is to be represented by different line models, including the constant parameter line model, the $\pi$-circuit and the new line model. The main and auxiliary contacts of the circuit breaker close at times specified in [65] for an unfaulted line energization case, between 7.15 and $15.85 \mathrm{~ms}$. A single-phase to ground fault is assumed to occur in phase A at $10 \mathrm{~ms}$. From the field test results for the case of the unfaulted line energization, we found that the constant parameter line model provides results which come close to the field test results [65]. The performance of the new line model for the case of the faulted line energization is compared with the response of the constant parameter line model.

The response of the constant parameter line model is shown in Figure 4.9. The travel time $\tau$ of the short line is $4.31 \mu \mathrm{s}$. The simulation step size is chosen to be $4 \mu \mathrm{s}$, as the constant parameter line model requires that the simulation step size $\Delta t$ should not be larger than the travel time $\tau$ of the shortest line.

Figure 4.10 shows the response produced by the new line model for a step size $\Delta t$ slightly larger than the travel time $\tau$ of $4.31 \mathrm{~s} \mu$. From Figure 4.10, we can see that the response of the new line model agrees very well with that of the constant parameter line model.

The response of the nominal $\pi$-circuit line model is shown in Figure 4.11. This response is simulated for the same step size as for the new line model. From Figure 4.11, we can observe that the response of the nominal $\pi$-circuit line model does not match the results of the 
constant parameter line model. Also, it fails to duplicate the maximum peak overvoltage which are shown for both the new line model and the constant parameter line model. This is because the nominal $\pi$-circuit model is not accurate enough to represent the high frequency transients involved in the switching action.

From these results we can see that the new line model is more accurate than the $\pi$-circuit for the representation of short lines.

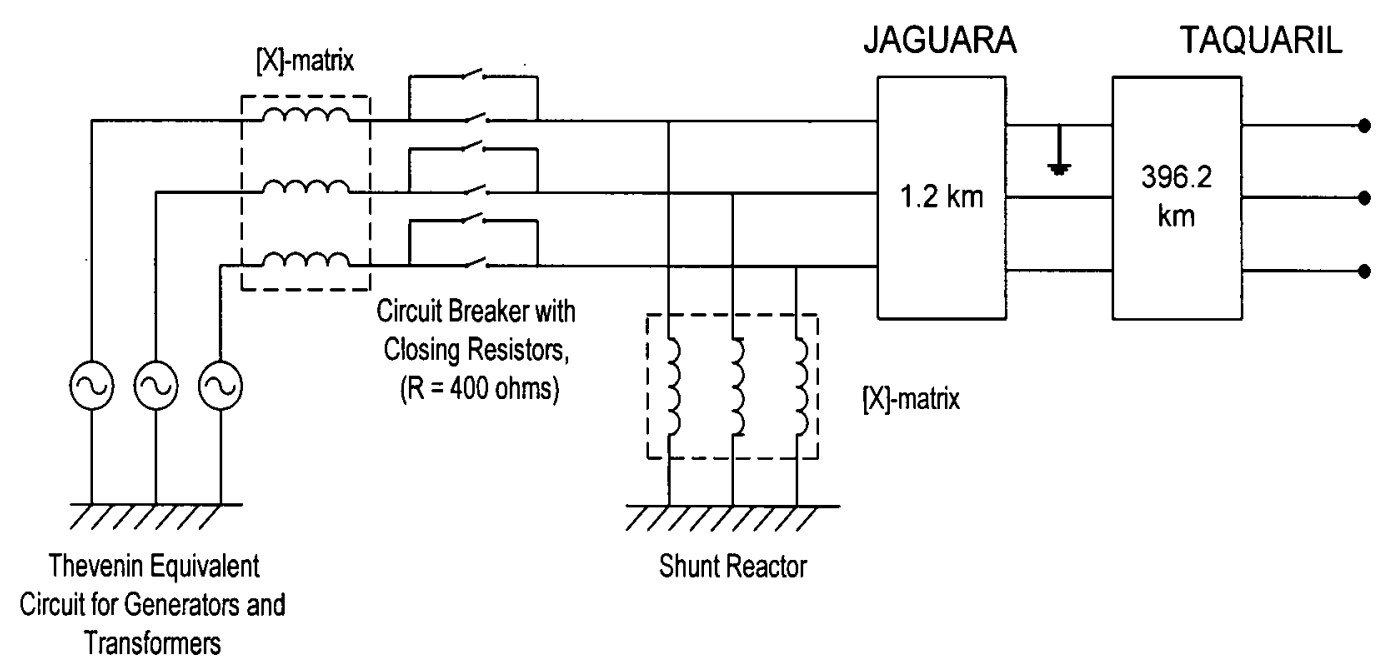

Figure 4.8 Transmission line energization 


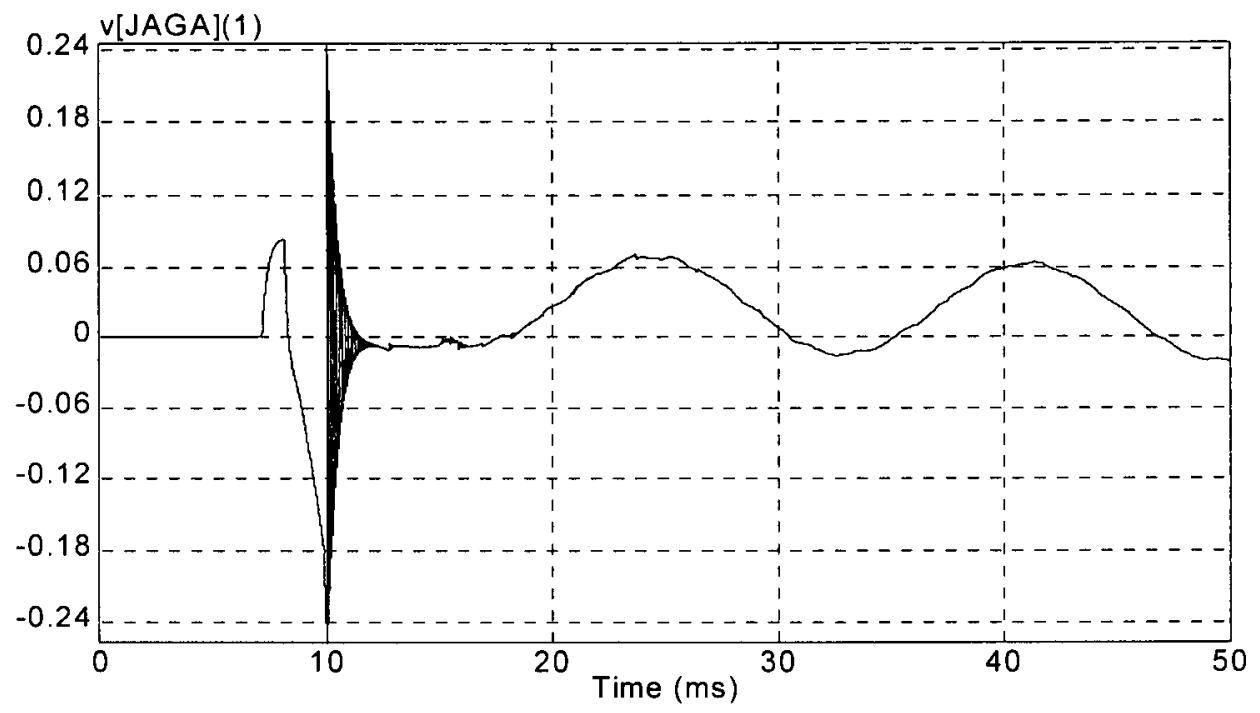

Figure 4.9 The constant parameter model response

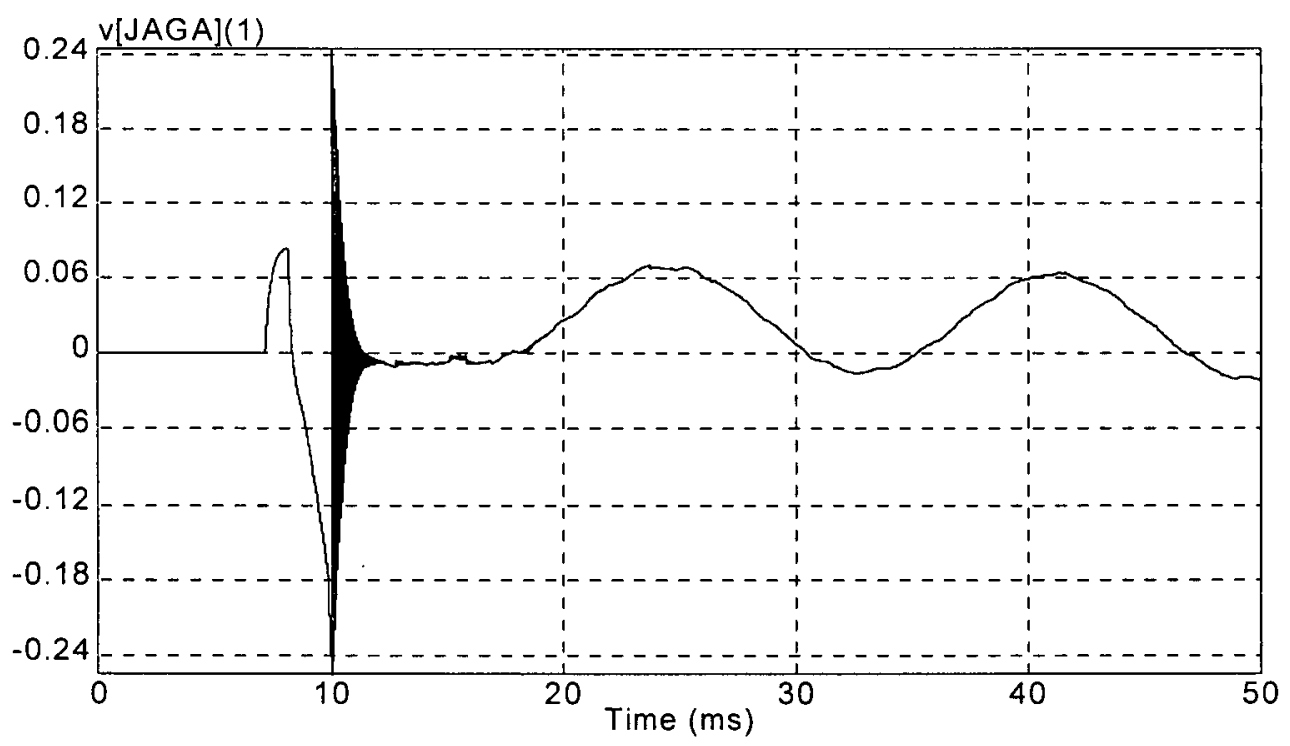

Figure 4.10 The new line model response 


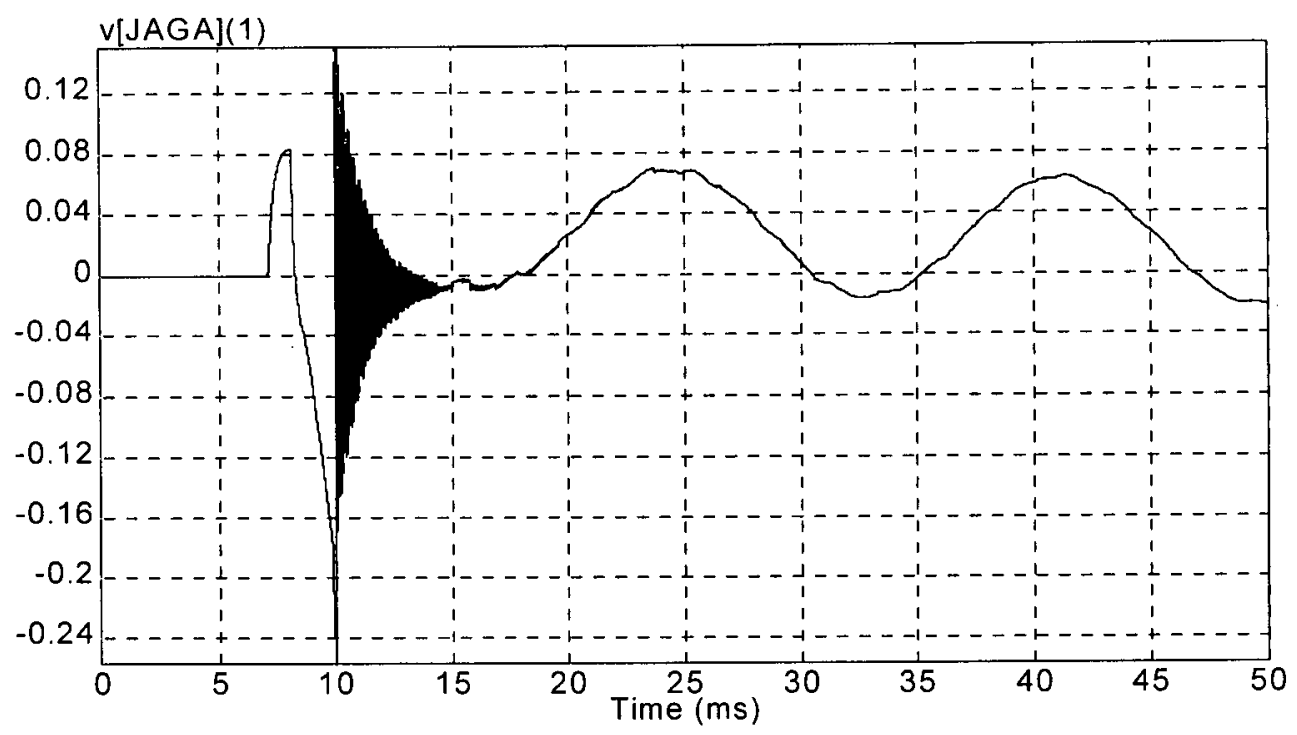

Figure 4.11 The pi-circuit line model response

\subsubsection{Power Electronics Case Study}

Power electronics devices are becoming very common in industrial power systems, for example in adjustable speed drives (ASD) which are often found in oil exploitation and mining [65]. ASD's are used because they allow a more flexible motor operation, above and below rated speed. ASD's generate harmonics, which are analyzed in this study [80].

The drive system studied is a square wave inverter with $480 \mathrm{~V}$ output. It has a step-up transformer 480:1350V, with a $135 \mathrm{HP}$ motor rated $950 \mathrm{~V}$. The motor is connected through a $2.5 \mathrm{~km}$ long submarine cable. Since the converter is operating at a very low switching frequency, low harmonics are generated. The basic components of this adjustable drive system are shown in Figure 4.12. 
The step-up transformer at the inverter is used because the semiconductor switches are, in this type of system, usually of low voltage rating. The power supply was represented by a voltage source behind a reactance. This representation is permissible when the drive system represents a small load compared to the short-circuit level of the supply system. If this is not the case, a frequency-dependent network would have to be used, or a detailed generator model in case of an isolated pumping system. The converter can be divided into three parts: rectifier, DC link, and inverter. The semiconductors in the rectifier are either diodes or thyristors, depending on the inverter type (voltage or current) and on the switching strategy, Pulse Width Modulation (PWM) or Pulse Amplitude Modulation (PAM).

This implies that the input voltage (or current) in the inverter will contain characteristic harmonics of the diode or thyristor bridge, together with the characteristic harmonics generated by the inverter. The DC link has the function of filtering the rectifier output, so as to produce as much as possible a ripple free voltage (or current).

The inverter has switches which are capable of conducting and interrupting the current at predetermined times. They are mostly Insulated Gate Bipolar Transistors (IGBT) with antiparallel diodes. The harmonics on the inverter side are reflected through the DC link to the converter input.

This leads to inter-harmonics, which may coincide with a multiple (or sub-multiple) of the supply frequency during some specific operating frequencies. Harmonic instability can arise when anti-resonances in the supply system coincide with a multiple or sub-multiple of the 
resonance in the drive system.

The aforementioned phenomena stress the importance of representing both converter stages (rectifier and inverter). Besides small resistances to account for conduction losses, all semiconductors were treated as ideal. Experience has shown that, for studies of the complete drive system, the modelling with ideal switches is sufficiently accurate in MicroTran, because this version uses the Critical Damping Adjustment (CDA) scheme to eliminate numerical oscillations. Other EMTP versions may require more complicated modelling to dampen numerical oscillations.

Figure 4.13 shows the voltage waveform of the field test recording conducted at the given drive system, at the sending end of the submarine cable between phase A and B. The field test waveform is asymmetric due to grounding problems while measuring the voltage. These measurements were done on an oil platform where fluctuations can occur in the ground voltage [80].

Figure 4.14 shows the simulation results with the new line model used for the representation of the submarine cable. The travel time for the short cable is $18.31 \mu \mathrm{s}$, while the simulation step size is chosen to be $30 \mu \mathrm{s}(\Delta t>\tau)$. From Figure 4.14 we can observe that the proposed model gives very reasonable answers. The filtering effect for high frequencies seems to be similar to the damping effect caused by skin effect in the actual cable.

Figure 4.15 show the simulation results for the $\pi$-circuit line model. This response is 
simulated with the same step size of the new line model of $30 \mu \mathrm{s}(\Delta t>\tau)$.

Figure 4.16 show the simulation results for the constant parameter line model for a step size of $18 \mu \mathrm{s}(\Delta t<\tau)$.

When we compare the field test recording of Figure 4.13 with the responses of the $\pi$-circuit and the constant parameter line models, we can observe that the responses of these two models are unrealistic. This is because the new line model has a filtering effect for high frequencies similar to the damping effect caused by skin effect in the actual cable. The new line model does not have the step size constraint of the constant parameter line mode, which is especially important for short lines and cables.

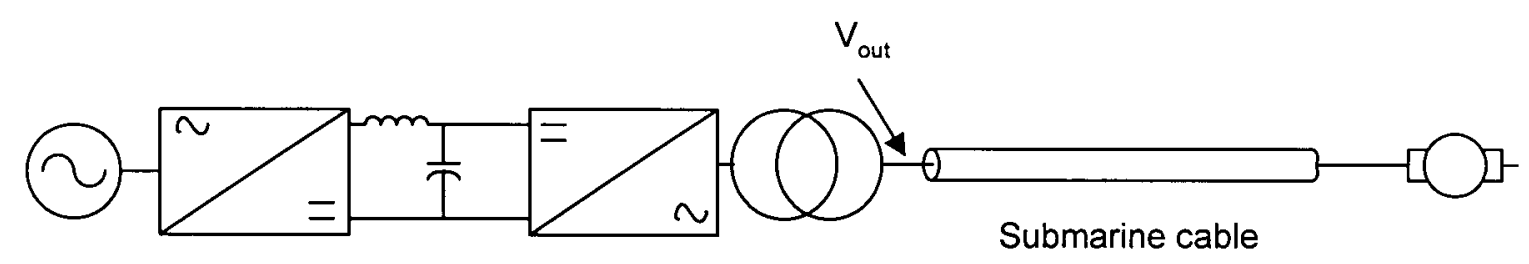

Figure 4.12 The power electronics case 


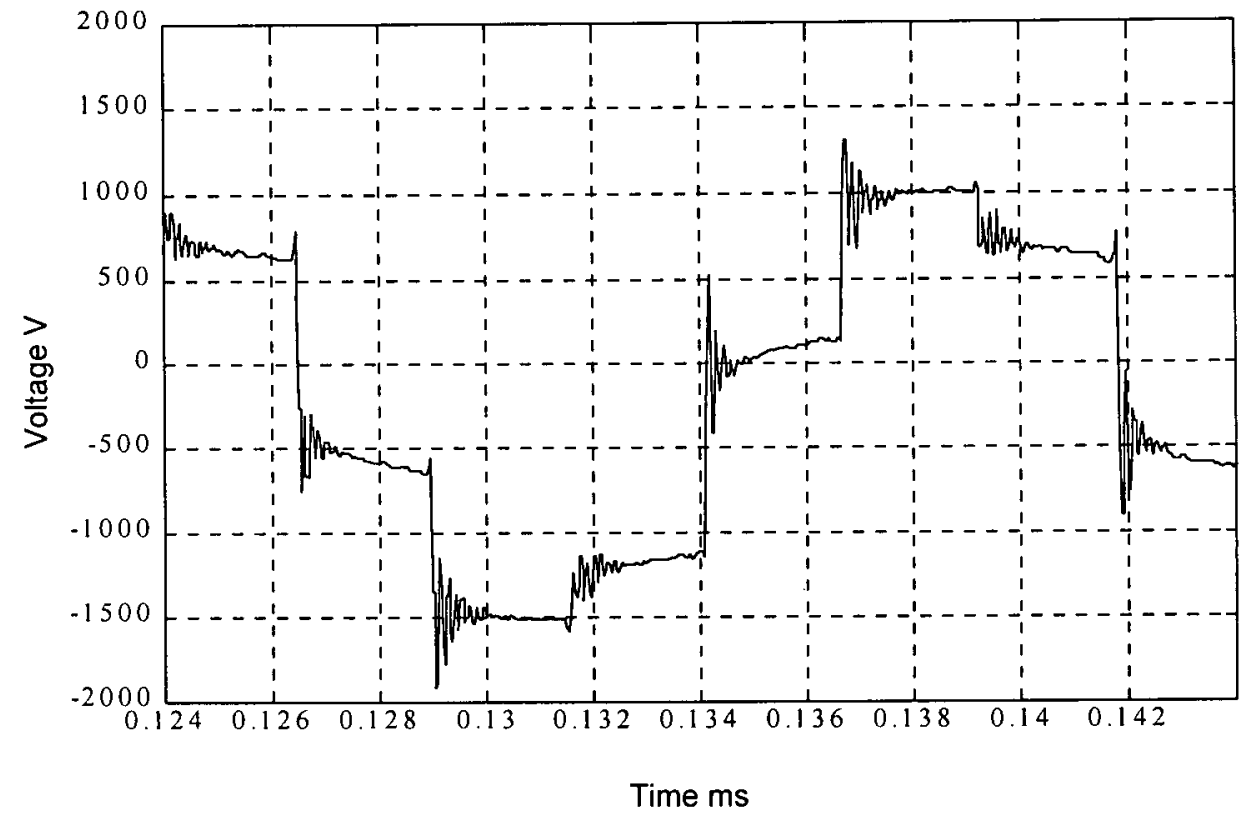

Figure 4.13 The field test recording for the drive system

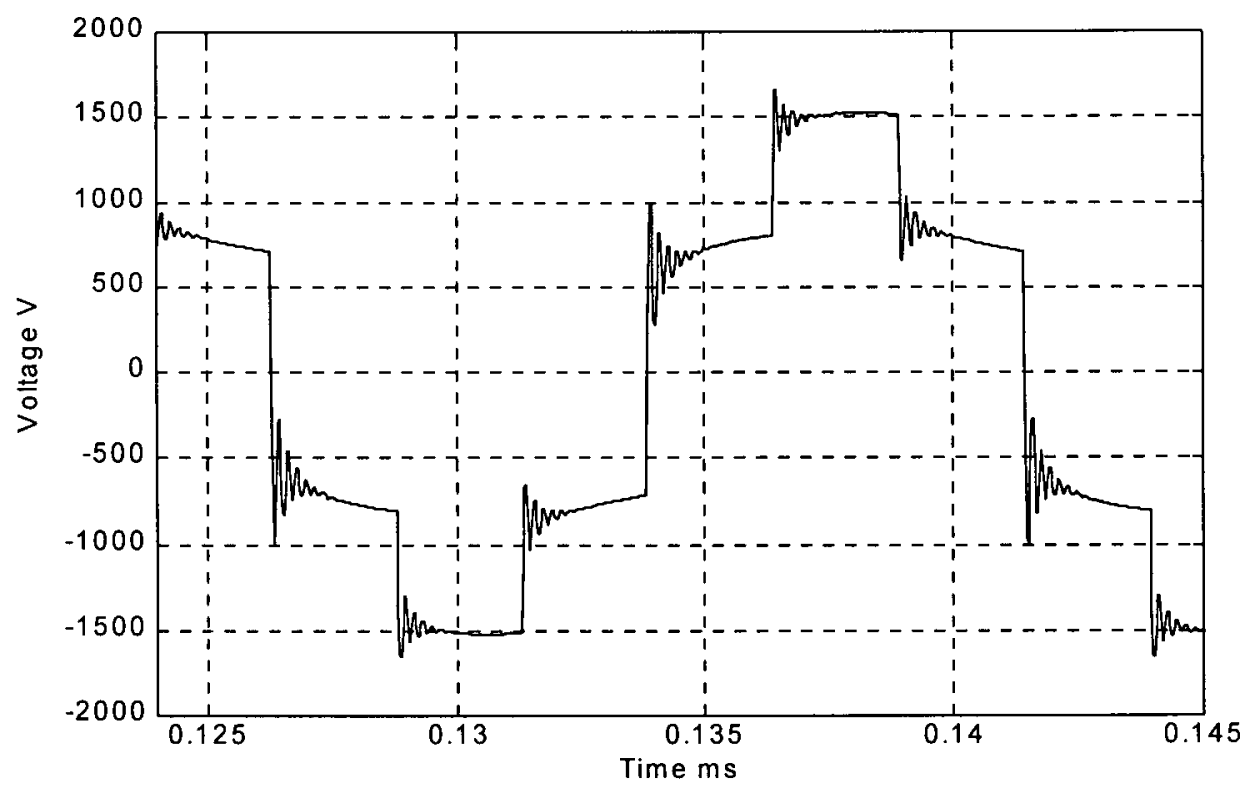

Figure 4.14 Simulation results for the new line model 


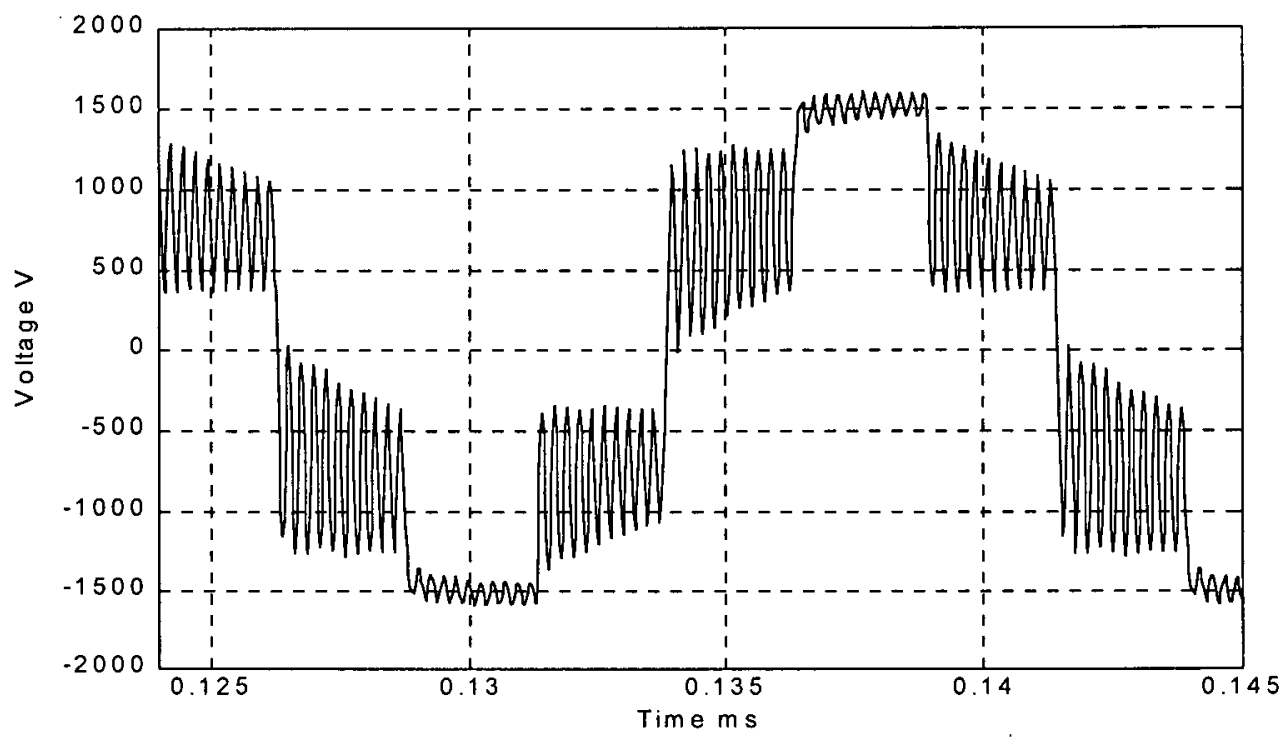

Figure 4.15 Simulation results for the $\pi$-circuit line model

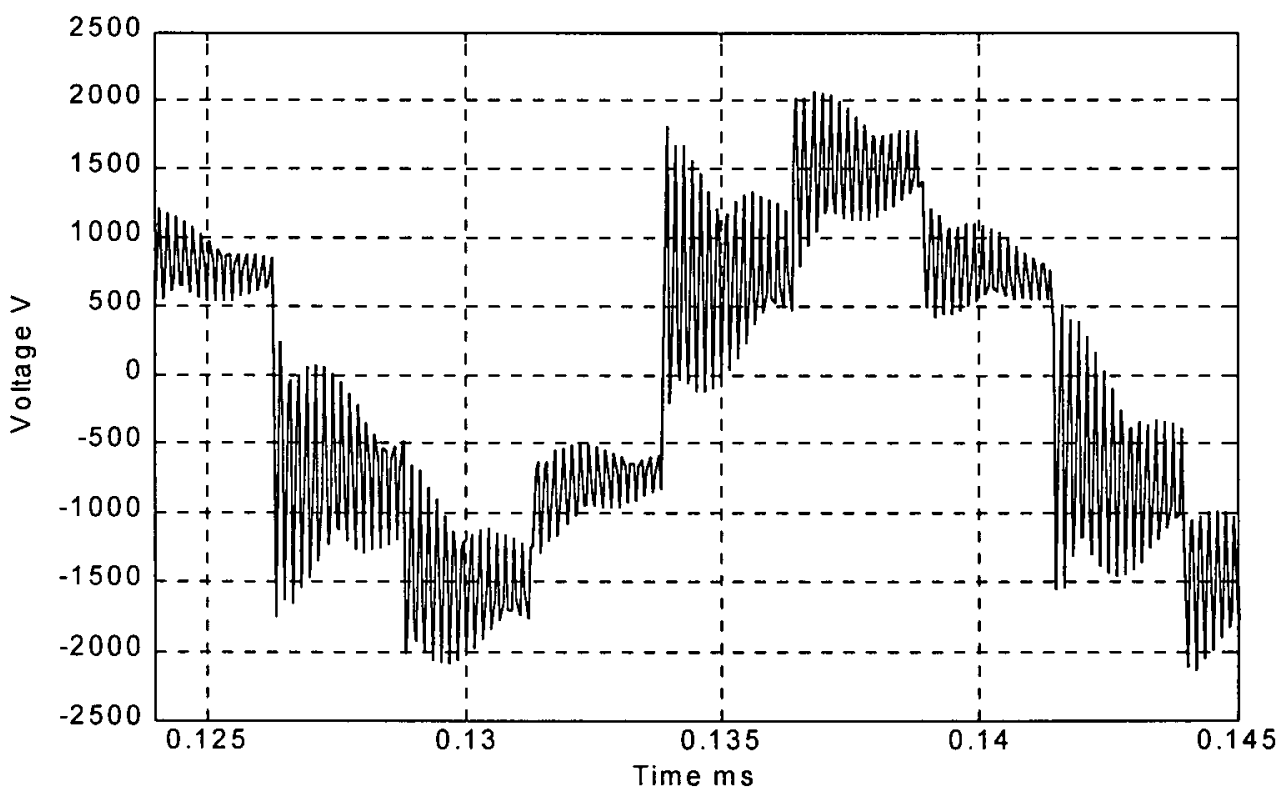

Figure 4.16 Simulation results for the constant parameter model 


\subsection{Summary}

This chapter describes a new transmission line model for the representation of short transmission lines and cables. This model is an extension of the constant parameter line model of the EMTP. It overcomes the limitation of requiring a time step size not larger than the travel time. This new line model can represent the transient phenomena accurately in the low frequency region. The new line model was tested with two practical case studies. Simulation results show that the new line model may provide more accurate answers for transient studies as compared with the nominal $\pi$-circuit line model as well as the constant parameter line model. 


\section{Chapter 5}

\section{Conclusions and Future Work}

\subsection{Overview}

The EMTP is widely used for simulating fast transient effects in electric power systems. However, using the EMTP and similar programs is not easy. Although the use of the program may be made more user-friendly with customized pre-/post-processors, such as graphical user-interfaces, the difficulty of proper modeling of the target power system remains basically unchanged. This is because of the high level of expertise required for the analysis of a large variety of transient phenomena.

In the preceding chapters, the research objectives of developing an EMTP support system for the simulation and analysis of power system transients were outlined, and the implementation of the proposed prototype intelligent system has been presented in more detail. Also, a 
knowledge base for switching surge overvoltages has been introduced. Finally, a new EMTP line model for representing short transmission lines and cables has been implemented. This line model was developed to overcome the step size constraint of the constant parameter and frequency dependent line models in the EMTP.

The conclusions and recommendations for future work are presented in the following sections.

\subsection{Conclusions}

The main accomplishments of this research are summarized below:

\subsubsection{The Prototype Intelligent Support System}

This dissertation has introduced an intelligent support system that supports the users of the EMTP in studying diverse transient phenomena in electric power systems. The support system assists the users to choose a base case data from a case database that contains various simulation cases as a starting point. When the modifications have been made to the base data to meet the user's particular purpose, the support system checks the validity of the data. Through the validation process the data set cannot only be made more appropriate, but the inexperienced user can learn the reasonable range of parameters and proper usage of the models. The results evaluation process of the support system can give hints to the user about the acceptable range of results obtained from the simulation. 


\subsubsection{Switching Surge Overvoltages}

This thesis has introduced a knowledge base for switching surge transients. From this knowledge base, some practical rules were derived for switching surge transients. The objective of this part of the thesis was to develop simple and approximate rules for the support system. These practical rules and the modelling suggestions were validated either by simulations or verified by interviews with EMTP experts. The modelling suggestions will help the EMTP user to select the proper model and to gain some insight into the case study being simulated.

\subsubsection{New Transmission Line Model}

This thesis described a new EMTP transmission line model for the representation of short overhead lines and cables. It overcomes the limitation of using a time step size not larger than the travel time. The error analysis for the short and open-circuit responses shows that the new line model has a filtering effect for higher frequencies. In a comparison with actual field test measurements, it could be shown that the new line model is suitable for a reasonable representation of cables, whereas both the $\pi$-circuit and the constant parameter line models produced unrealistic high frequency oscillations.

\subsection{Recommendations for Future Work}

The following are some recommendations for future directions of research activities for the development of a support system for electric power system transients.

1. The case database should be expanded to include more case data from different power system transient studies. 
2. The complete scheme of the Case-Based Reasoning (CBR) presented in [39][40] with indexing, case storage and retrieval, could be used to improve the design of the proposed case database.

3. The expanded data validation process should then help the user in selecting the proper models based on more rules derived from the knowledge base.

4. The results evaluation should be expanded to provide the user with more modeling experience in the overall picture of the simulated system. Also, the information presented in Figure 4.1 about overvoltages magnitudes for different system conditions could be for the results evaluation process.

5. The existing knowledge base should be expanded to include more types of transient case studies. The derived practical rules would be used in the proposed EMTP support system.

6. The new line model with $\Delta t \geq \tau$ should be extended to the more accurate frequency dependent line model which has been used extensively for cases with $\Delta t<\tau$, and from single-phase to multi-phase models. 


\section{Bibliography}

[1] CIGRE Working Group 13-02 Switching Surges Phenomena in EHV Systems, "Switching Overvoltages in EHV and UHV Systems with Special Reference to Closing and Reclosing Transmission Lines," Electra, Vol. 30, pp 70-122, 1973.

[2] CIGRE Working Group 13.05, "The Calculation of Switching Surges (I). A Comparison of Transient Network Analyzer Results," Electra, Vol. 19, pp. 67-78, 1971.

[3] CIGRE Working Group 33.02, "Guidelines for Representation of Network Elements When Calculating Transients", Paris, 1990.

[4] H. W. Dommel, EMTP Theory Book, Microtran Power System Analysis Corporation, Vancouver, B.C., Canada, 1992.

[5] H. W. Dommel, "Digital Computer Solution of Electromagnetic Transients in Single- and Multiphase Networks," IEEE Trans. on Power Apparatus and Systems, Vol. PAS-88, No. 4, pp. 388-399, April 1969. 
[6] C.A.F. Cunha and H.W. Dommel, "Computer simulation of field tests on the $345 \mathrm{kV}$ Jaguara-Taquaril line", (in Portuguese), Paper BH/GSP/12, presented at iII Seminario Nacional de Producao e Transmissao de Energia Eletrica" in Belo Horizonte, Brazil, 1973 (Portuguese paper or English translation by D.I. Cameron, available from H.W. Dommel).

[7] H. W. Dommel, Case Studies for Electromagnetic Transients, second edition, Microtran Power System Analysis Corporation, September 1993.

[8] Electromagnetic Transients Program (EMTP), EMTP Field Test Comparisons, EPRI Report EL-6788s, 1990.

[9] L. Bergeron, Water Hammer in Hydraulics and Wave Surges in Electricity; ASME Committee, John Wiley \& Sons, Ltd., New York, 1961.

[10] H. W. Dommel, MicroTran Reference Manual, Microtran Power System Analysis Corporation, Vancouver, B.C., Canada, 1992.

[11] Transmission Line Reference Book $345 \mathrm{kv}$ and Above, Electric Power Research Institute (EPRI), 1975.

[12] Electrical Transmission and Distribution Reference Book, Westinghouse Electric Corporation, 1964.

[13] Klaus Ragaller, "Surges in High-Voltage Networks", Plenum Press, New York, 1980.

[14] IEEE Working Group 15.08.09, "Modeling and Analysis of System Transients Using Digital Program", IEEE PES Special Publication, 1998. 
[15]P.M. Anderson and R.G. Farmer, Series Compensation of Power Systems, Book, 1st edition, PBLSH, California, 1996.

[16] Dai Nan, Processing Tools for Transient Simulation Programs at UBC, M.S. Thesis, October 1999.

[17] J. A. Martinez-Velasco, Computer Analysis of Electric Power System Transients, Selected Readings, IEEE, 1997.

[18] A. Inoue and S. Kanao, "Observation and Analysis of Multi-phase Grounding Faults Caused by Lightning", IEEE Transactions on Power Delivery, Vol. 7, No. 2, pp 12051215, January 1996.

[19] Y. Matsumoto et al., "Measurements of Lightning Surges Transmission Line Equipped with Arresters Struck by Natural and Triggered Lightning", IEEE Transactions on Power Delivery, Vol. 11, No. 2, pp 996-1002, April 1996.

[20] J. A. Hallady and C.H. Shin, "Resonant Overvoltage Phenomena Caused by Transmission Line Fault', IEEE Transactions on Power Apparatus and Systems, Vol. 104, No. 9, pp 2531-2539, September 1985.

[21] M. M. Adibi, R. W. Alexander and B. Avramovic, "Overvoltage Control During Restoration", IEEE Transactions on Power Systems, Vol. 7, No. 4, pp 1464-1470, November 1992.

[22] F. Alvarado, R. Lasseter, and Y. Liu “An Integrated Engineering Simulation Environment," IEEE Trans. on Power Systems, Vol. 3, No. 1, pp. 245-253, February 1988. 
[23]J. Lee, R. Lasseter, and J. Beetem, "A Graphic Interface for Power System Simulation," Proceedings of the 1993 IEEE Power Industry Computer Application Conference (PICA), Vol. No. 1, pp. 269-276, May 1993.

[24]R. Lasseter "Hierarchical Interface Eases Power System Simulation," IEEE Computer Applications in Power Magazine, Vol. No. 8, pp.29-31, January 1995.

[25] Alternative Transient Program "ATP" Rule Book, printed by the Leuven EMTP Center (LEC), July, 1987.

[26] J. Martinez, "How to Adapt The EMTP for Classroom Instruction," IEEE Trans. on Power Systems, Vol. 7, No. 1, pp. 351-359, February 1992.

[27]D. Leonard and D. Wilcox, "Development of a New EMTP Using MATLAB," Proceedings of The $29^{\text {th }}$ Universities Power Engineering Conference, University College, Galway, Ireland, Vol. No. 2, pp. 641-644. September 1994.

[28] MATLAB User Guide, The Math Works Inc., 1993

[29] ATPDRAW- Graphical Preprocessor to ATP, Version 2.0, 1994.

[30]E. Feigenbaum, "The Art of Artificial Intelligence: Themes and Case Studies of Knowledge Engineering," IJCAI, Vol.5, pp. 1024-1029, 1977.

[31]F. Hayes-Roth, "The Knowledge Base Expert System: A Tutorial," Computer, IEEE, September 1984.

[32] J. Wentworth, "Developing expert systems", OECD Workshop on Knowledge- based Expert Systems in Transportation, Espoo, Finland, pp. 36-77, 1990. 
[33] S. Kumar, et al,. "An Expert System for Trouble-Shooting and Quality Problems in Continuos Casting of Steel Billets," FAC, Workshop on Expert Systems in Mineral and Metal Processing, Helsinki, Finland, 1991.

[34] T. Dillon, and M. Laughton, Expert System Applications in Power Systems, Prentice Hall International, 1990.

[35] A Tutorial Course on: "Knowledge-Based Systems Techniques with Applications to Power Systems", IEEE Power Engineering Society, IEEE Catalog No. 93 EHO 387-1PWR, October 1993.

[36] Z. Zhang, G. Hope, and O. Malik, "Expert Systems in Electric Power Systems - A Bibliographical Survey," IEEE Trans. on Power Systems, Vol. 4, No. 4, pp. 1355-1361, October 1989.

[37] A. Germond and D. Niebur, "Survey of Knowledge-Based Systems in Power Systems:Europe,", Proceedings of The IEEE, Vol.80, No. 5, pp. 732-744, May 1992.

[38] CIGRE Report "Application of Expert Systems to Education and Training of Power System Engineers", TF 38.06.05, Electra, Vol. No. 165, pp. 97-127,April 1996.

[39] J. Hseih and C. Liu, "An Integrated Knowledge- and Algorithm- Based Method for Power Converter Design," IEEE Power Electronics Specialists Conference Record, pp. 13161323, March 1992.

[40] J. Hseih and C. Liu, "Intelligent System as a Computer Aid for Power Converter Designs," Engineering Intelligent Systems, Vol. 1, No. 1, pp. 21-30, June 1993. 
[41] M. Huneault, et al, "A Study of Knowledge Engineering Tools in Power Engineering Applications," IEEE Trans. on Power Systems, Vol. 9, No. 4, pp. 1825-1832, November 1994.

[42] J. Martin and S. Oxman, Building Expert Systems- A Tutorial, Prentice Hall, 1988.

[43] Young D., et al, "Development of a Practical Expert System for Alarm Processing" IEE Proceedings-C, Vol. 139, No. 5, pp. 437-447, Sept.1992.

[44] Artificial Intelligence Section, CLIPS Reference Guide, CLIPS Version 6.0, Lyndon B. Johnson Space Center, 1993.

[45] Knowledge Systems Laboratory, Fuzzy CLIPS User's Guide, Fuzzy CLIPS Version 6.02A, Institute for Information Technology, National Research Council Canada, 1994.

[46] W. Mettrey, "A Comparative Evaluation of Expert System Tools", IEEE Transactions Computer Magazine, pp. 19-31, February 1991.

[47] W. Mettrey, "Evaluation of AI Languages and Knowledge Engineering Environments", Northern Telecom Report KBS-88-009, Research Triangle Park, NC, 1988.

[48] W. Mettrey, "An Assessment of Tools for Building Large Knowledge-Based Systems", AI Magazine, Vol. 8, No. 4, pp. 81-89, Winter 1987.

[49]L. Brownston et al., Programming Expert Systems in OPS5, An Introduction to RuleBased Programming, Addison Wesley, Reading. Mass., 1985.

[50] COMDALE/X user's manual, Comdale Technologies (Canada) Inc., 1986-1993.

[51]T. Niimura, H.W. Dommel, J.R. Marti, "An Intelligent Guide to Support EMTP Simulation", Stockholm Power Tech. Conference, June 1995, Stockholm, Sweden. 
[52] A. Ibrahim T. Niimura, H.W. Dommel, J.R. Marti, "Case-based Approach for Transient Analysis Modeling Using EMTP”, The International Conference on Power Systems Transients, Seattle, Washington, June 1997.

[53] F.L Alvarado, et al., EMTP Workbook, Vol. 1-3, EPRI Report EL-4651s, 1986-89.

[54] Electromagnetic Transients Program (EMTP), Version 2.0: Revised Application Guide, EPRI Report EL-7321s, 1991.

[55] A. Greenwood, Electrical Transients in Power Systems, John Wiley \& Sons, 1991.

[56] M. El-Hawary, Electric Power Systems, Reston Publishing Company, 1983.

[57] A. Ibrahim, M. Salama, "Frequency Dependent Network Equivalents for Electromagnetic Transient Studies", The International Journal of Electrical Power \& Energy Systems, Vol. 21, pp. 395-404, August 1999.

[58] A. Ibrahim, M.M.A. Salama, "Frequency Dependent Network Equivalent for AC Power System Using the QZ Algorithm, "IEEE Canadian Conference on Electrical and Computer Engineering CCECE/CCGEI'95, Montreal, Canada, Vol. No. 3/4, 1995, pp. 56- 59. [59] A. Ibrahim, M.M.A. Salama, "Frequency Dependent Network Equivalent Algorithm for AC Power Systems, "IEEE Canadian Conference on Electrical and Computer Engineering CCECE/CCGEI'96, Calgary, Canada, Vol. No. 2, 1996, pp. 639-642.

[60]F.J. Marcano, Modelling of Transmission Lines Using Idempotent Decomposition, M.S. Thesis, The University of British Columbia, Department of Electrical and Computer Engineering, Vancouver, BC, Canada, August 1996 
[61] J. Marti, "Accurate Modelling of Frequency-Dependent Transmission Lines in Electromagnetic Transients Simulations," IEEE Trans. on Power Apparatus and Systems, Vol. PAS-101, pp. 147-157, January 1982.

[62] F. Castellanos, J. Marti, "Full Frequency-Dependent Phase-Domain Transmission Line Model," IEEE Trans. on Power Systems, Vol. 12, No. 3, pp. 1331-1339, August 1997.

[63] W. Meyer, H. Dommel, "Numerical Modelling of Frequency Dependent Transmission Line Parameters in an Electromagnetic Transients Program," IEEE Trans. on Power Apparatus \& Systems, Vol. PAS-93, pp. 1401-1409, September/October 1974.

[64] S. Henschel, A. I. Ibrahim, H. W. Dommel, "Transmission Line Model for Variable Step size Simulation Algorithms", The International Journal of Electrical Power \& Energy Systems, Vol. 21, pp. 191-198, January 1999.

[65] A. Lima, R. Stephan, and A Pedroso," Modelling the Electrical Drive System for Oil Exploitation," International Power System Transients Conference, pp. 234-239, June 1997.

[66]H. V. Nguyen, Simulation of Lighting Surges on Transmission Lines, Ph.D. Thesis, The University of British Columbia, Department of Electrical and Computer Engineering, Vancouver, BC, Canada, February 1996.

[67]L. Marti, Simulation of Electromagnetic Transients in Underground Cables with Frequency Dependent Modal Transformation Matrices, Ph.D. Thesis, The University of British Columbia, Department of Electrical and Computer Engineering, Vancouver, BC, Canada, November 1986. 
[68]H. W. Dommel and J.R. Marti, "Overhead Transmission Line Models for Steady State and Transient Analysis," The Canadian Electrical Association, Power Planning \& Operation Section, March 1985.

[69] K. Tanaka, An Introduction to Fuzzy Logic for Practical Applications, Springer-Verlag, 1997.

[70]A. Ibrahim, T. Niimura, H. Dommel, "An Intelligent Support System for the Analysis of Electric Power System Transients" The International Conference on Intelligent Systems and Control, June 1-3, 1998, Halifax, Canada.

[71]A. Ibrahim, D. Lindenmeyer, T. Niimura, H. Dommel, "An Intelligent Support System for the Results Evaluation Analysis of Electric Power System Transients" IEEE Canadian Conference CCECE/CCGEI'98, Waterloo, Canada, Vol. No. 1, May 24-28,1998.

[72] A. Ibrahim, S. Henschel, H. W. Dommel, T Niimura, "Transmission Line Model For Large Step Size Simulations", The IEEE Canadian Conference CCECE/CCGEI'99, Edmonton, Alberta, Canada, May 1999.

[73] A. Ibrahim, S. Henschel, A. C. Lima, H. Dommel, T. Niimura, "A New EMTP Line Model For Short Overhead Lines And Cables ", Submitted to the IEEE Transactions on Power Delivery.

[74] A. Ibrahim, T. Niimura, H. Dommel, "An Intelligent Support System for the Analysis of Electric Power System Transients", Submitted to The International Journal of Intelligent Systems and Control. 
[75] J. Mahseredjian and F. Alvarado, "Creating an Electromagnetic Transients Program in Matlab: MatEMTP," IEEE/PES Winter Meeting, Paper 96 WM 098-4 PWRD, January, 1996.

[76] O. Nayak, G. Irwin and A. Neufeld, "GUI Enhanced Electromagnetic Transients Simulation Tools" IEEE Computer Applications in Power, Vol. 8, No. 1, pp. 17-22, January 1995.

[77] R. S. Rosales, Simulation environment for a real-time power system simulator, M.S. Thesis, The University of British Columbia, Department of Electrical and Computer Engineering, Vancouver, BC, Canada, 1997.

[78] Sebastian Henschel, Analysis of Electromagnetic and Electromechanical Power System Transients with Dynamic Phasors, Ph.D. Thesis, The University of British Columbia, Department of Electrical and Computer Engineering, Vancouver, BC, Canada, February 1999.

[79] H. W. Dommel, Notes on Power Systems Analysis, ELEC 463 Course Notes, The University of British Columbia, Department of Electrical and Computer Engineering, Vancouver, BC, Canada, 1975.

[80] A. Delima, H. Dommel, and R. Stephan, "Modelling Adjustable Speed Drives with Long Feeders", IEEE Transactions on Industrial Electronics, Vol. 47, No. 3, pp. 549-556, June 2000. 


\section{Appendix A}

\section{The Case Database}

The EMTP support system currently supports the following cases. Some of these cases are from the EMTP workbook [53]:

\section{Switching Transients:}

I. Overhead Line Energization

II. Overhead Line Re-energization with Trapped Charges

III. Capacitor Switching

IV. Cable Energization

V. Transformers and Shunt Reactor Energization

VI. Load Energization

\section{Fault Analysis:}


I. Single Line-to-Ground Fault of an Overhead Line

II. Single Line-to-Ground Fault of an Overhead Line with Frequency Dependence

III. Transient Stability Analysis with a Three-Phase Short-Circuit

IV. Load Rejection

3. Resonance Analysis:

I. Ferro-resonance Analysis

\section{A.1 Switching Transients Analysis}

The switching transients analysis menu of the EMTP support system case database has the following cases which will be described briefly.

\section{A.1.1 Overhead Line Energization}

The selection of the overhead line models depends on how accurate the transmission lines should be represented in terms of simulation step size. The following cases are included in the overhead line energization sub-menu:

\section{A.1.1.1 Energization of Transposed Line}

In this configuration shown in Figure A.1, a transposed three-phase transmission line is represented by the constant-parameter line model. The constant-parameter model is a distributed parameter line model without frequency dependence. If the effect of transmission line on overall transient phenomena is the matter of interest, you should model the transmission line by a distributed-parameter line model. On the other hand, the source circuit representations 
can be simplified. It may be more practical to assume a CB between Bus1 and the transmission line in the three-phase line energization of this example (There may be other load on the bus).

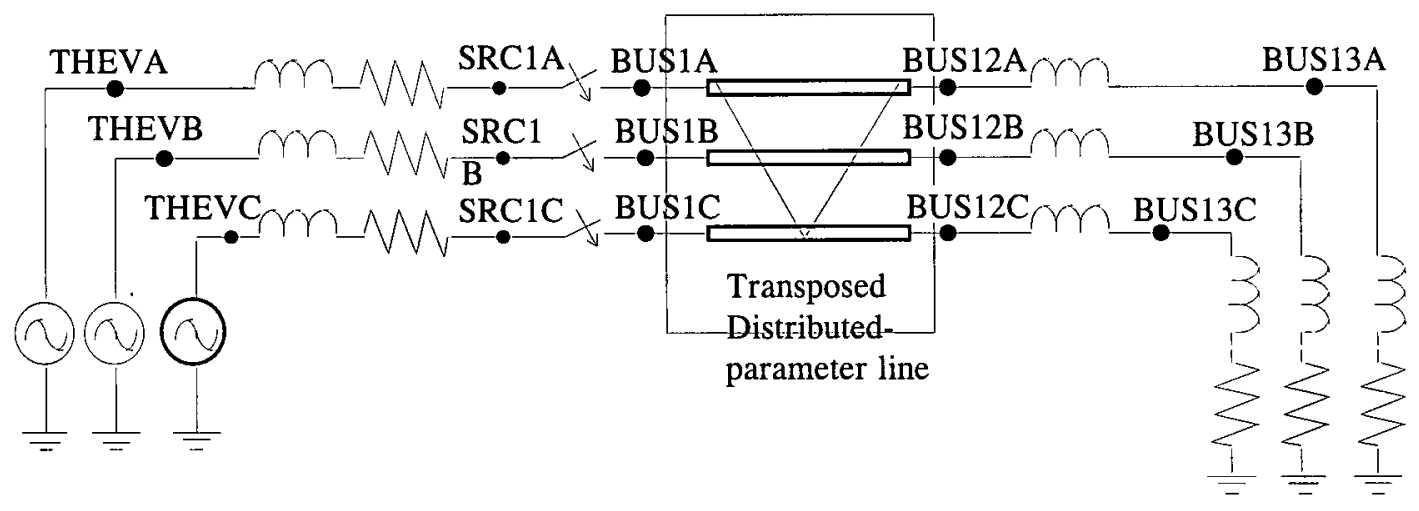

Figure A.1 Transposed transmission line.

\section{A.1.1.2 Energization of Untransposed Line}

If the transmission line is untransposed, the user must supply the modal transformation matrix. If the user has, for example, only positive- and zero-sequence parameters of the target circuit, he or she can use fdLine support program for modal parameters with detailed configuration of towers.

This sample power system shown in Figure A.2 includes a long overhead transmission line between BKR1 and Bus2. Bus 2 is assumed no-load, and the opening circuit breaker BKR1 leaves the unloaded (capacitive) line charged. The voltage sources are represented by Thevenin equivalents. The timing of breaker open or close action will influence the magnitude and polarity of trapped charges and the resultant overvoltage when reclosed. 


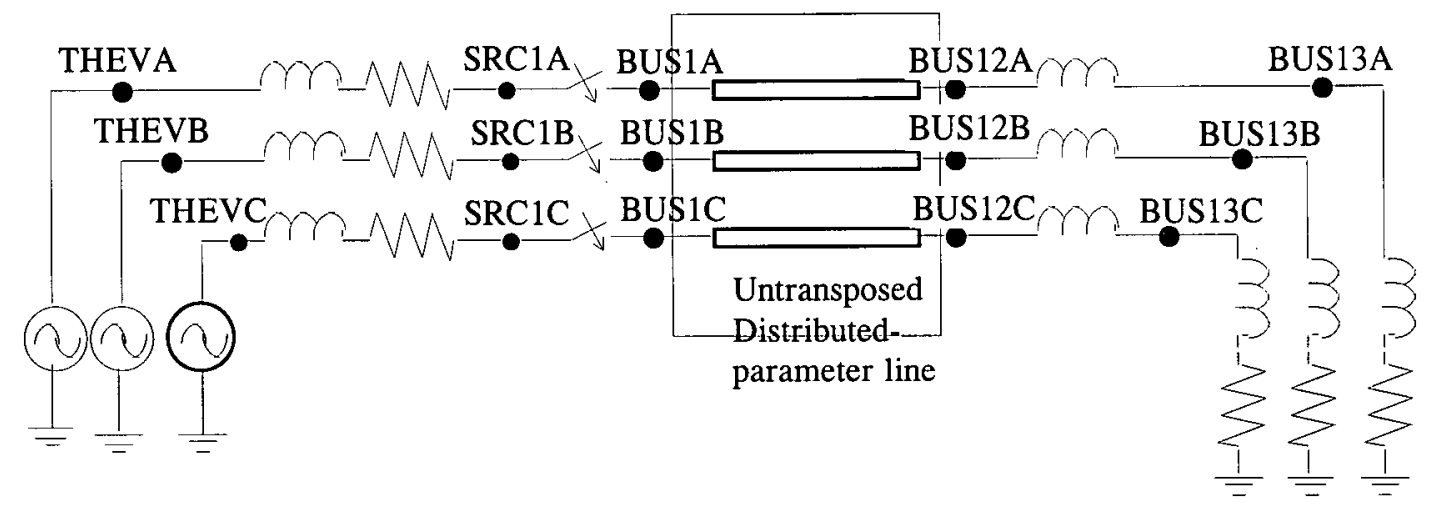

Figure A.2 Untransposed transmission line

\section{A.1.1.3 Reclosing into a Line with Trapped Charges}

This sample power system shown in Figure A.3 includes a long overhead transmission line between BKR1 and Bus2. Bus2 is assumed no-load, and the opening circuit breaker BKR1 leaves the unloaded (capacitive) line charged. The voltage sources are represented by Thevenin equivalents. The timing of breaker open or close action will influence the magnitude and polarity of trapped charges and the resultant overvoltage when reclosed. 


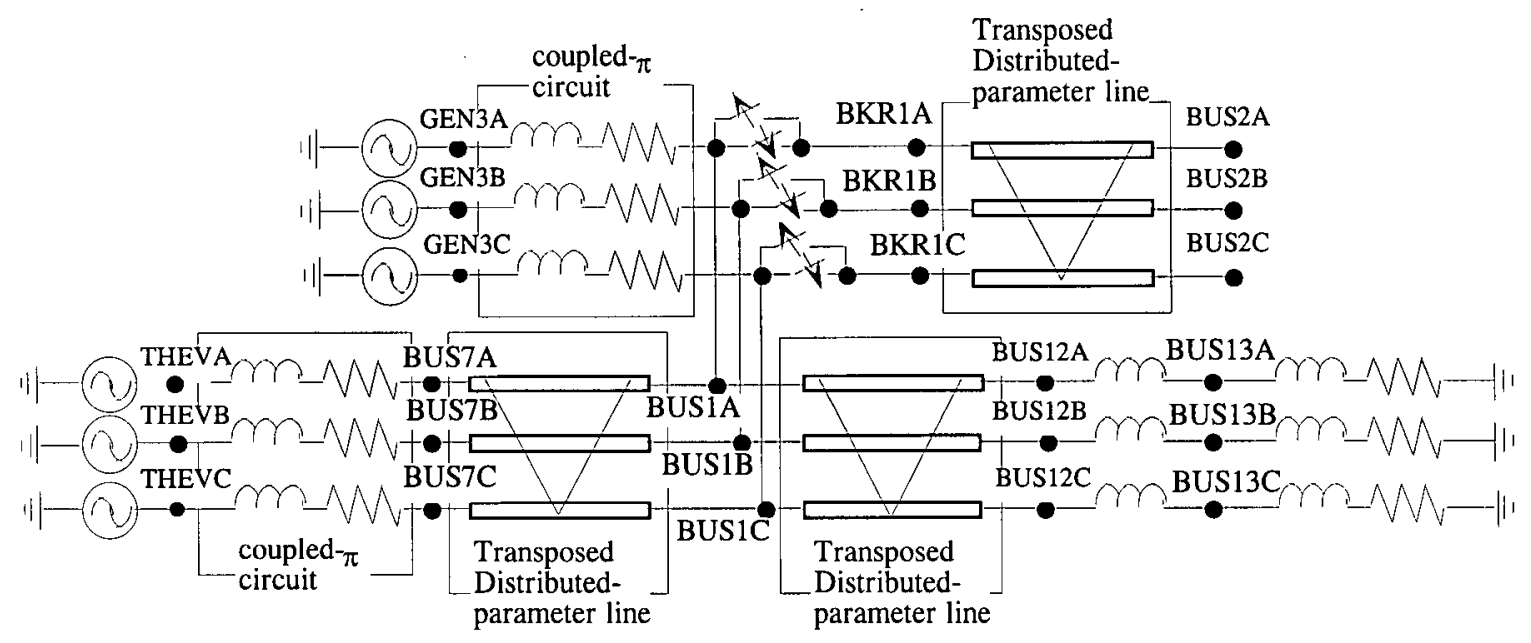

Figure A.3 Reclosing of overhead lines with trapped charges

\section{A.1.2 Capacitor Switching}

The main point in capacitor switching is:

a. Charging current upon energization.

b. Overvoltages by irregular breaker opening sequences (particularly, when breaker gets stuck). The capacitor switching sub-menu has the following cases:

\section{A.1.2.1 Capacitor Bank De-energization}

This example shown in Figure A.4 simulates the breaker opening actions when the threephase capacitor bank is energized by a three-phase source. The neutral grounding is considered by a separate capacitor. In this example, breakers are assumed to operate in correct sequence. 


\section{A.1.2.2 Capacitor Bank Re-energization with a Circuit breaker Stuck}

This example shown in Figure A.4 simulates the breaker opening actions when the threephase capacitor bank is energized by a three-phase source. The neutral grounding is considered by a separate capacitor. In this example, breaker in phase-b gets stuck. Breaker between BUSB and BANKB opening is delayed to half-cycle $(8.3 \mathrm{~ms})$ after the first zero current. This example simulates the breaker opening actions when the three-phase capacitor bank is energized by a three-phase source.

There is a recommendation on neutral grounding of capacitor banks in ANSI C37.99, pp.2425 (Guideline on protection relaying). Neutral grounding practice of capacitor banks are also summarized in the IEEE Standards. The neutral grounding branch is necessary for the EMTP. Without such branch the program stops due to zero diagonal element. It is reasonable to assume some stray capacitance in phase to ground.

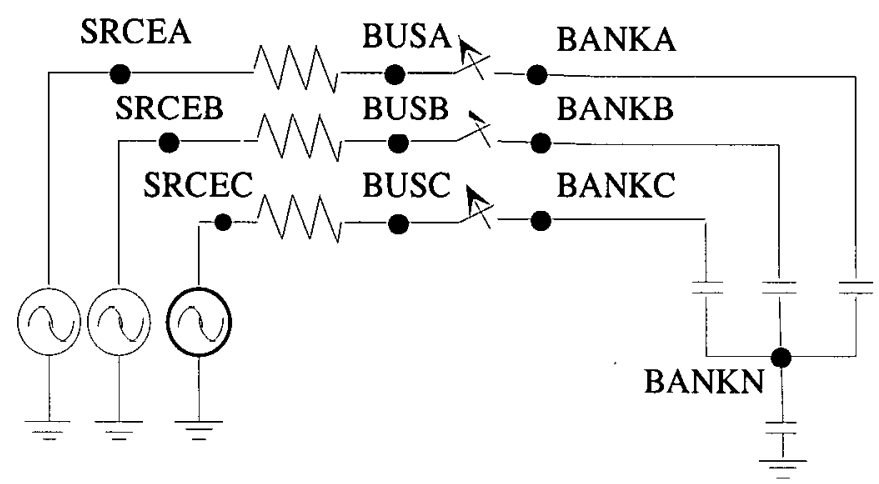

Figure A.4 Capacitor bank De-energization 


\section{A.1.2.3 Capacitor Bank De-energization with Transformers}

This sample network shown in Figure A.5 considers the de-energization of capacitor banks connected in parallel to transformer. Upon opening the breakers, transient recovery voltages across breaker contacts develop and it can result in breaker restrike. Also, the saturation of a transformer can lead to a resonant condition.

In a practice circuit configuration we consider the de-energization of capacitor banks connected in parallel to transformer.

Upon opening the breakers, transient recovery voltages across breaker contacts develop more than 2.0 p.u. This can result in breaker restrike. Also, the presence of a transformer can lead to a resonant condition due to transformer saturation.

Source impedance is represented by lumped R-L. The transformer is represented by ideal transformers and saturation is included on the low voltage side by piece-wise linear inductances (Type-93 for MicroTran). Phase-b breaker is assumed stuck for another cycle. Results may differ due to the residual flux in the transformer. 


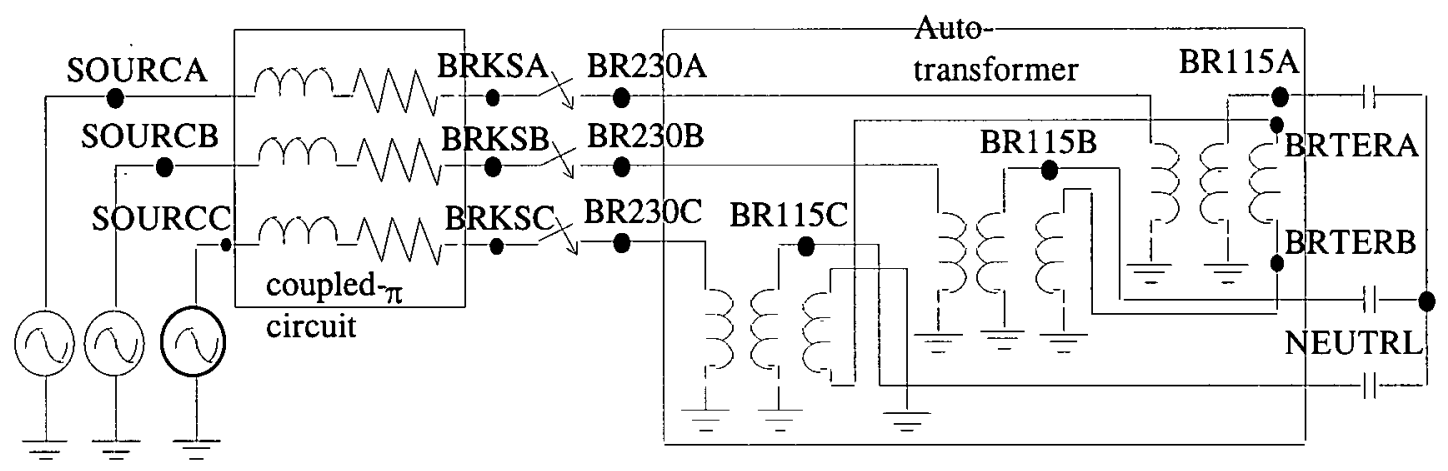

Figure A.5 Capacitor switching with transformers

\section{A.1.3 Cable Switching}

Cable modeling in the present MicroTran version of the EMTP is not resolved at this point. The difficulties are mainly due to frequency dependence and variety of connections of multiphase cables. The single-phase modeling of cables given here is only valid for submarine cables where we can neglect mutual coupling. Otherwise, we need to model mutual coupling of cables. $\pi$-models are practically inaccurate except for steady-state calculation.

fdLine support program may be used to obtain impedance for single-phase cables but so far there had not been such an attempt. All the following examples neglect the effect of frequency on modal parameters and modal transformation matrices.

\section{A.1.3.1 Three-Phase Cable Energization}

In this sample circuit shown in Figure A.6 cables are modeled as single-phase two mode lines (one sheath mode) assuming that all sheaths voltages are the same. This modeling neglects the mutual coupling of each phase. Such assumption is only valid for submarine cables. 


\section{A.1.3.2 Three-phase Cable Energization with Crossbonding}

In this example shown in Figure A.7 cables are assumed as crossbonded. By crossbonding the sheath of one phase in one cable section is connected to the sheath of a different phase in the next cable section. In the sample model, cables are represented by multi-sectioned $\pi$-circuits.

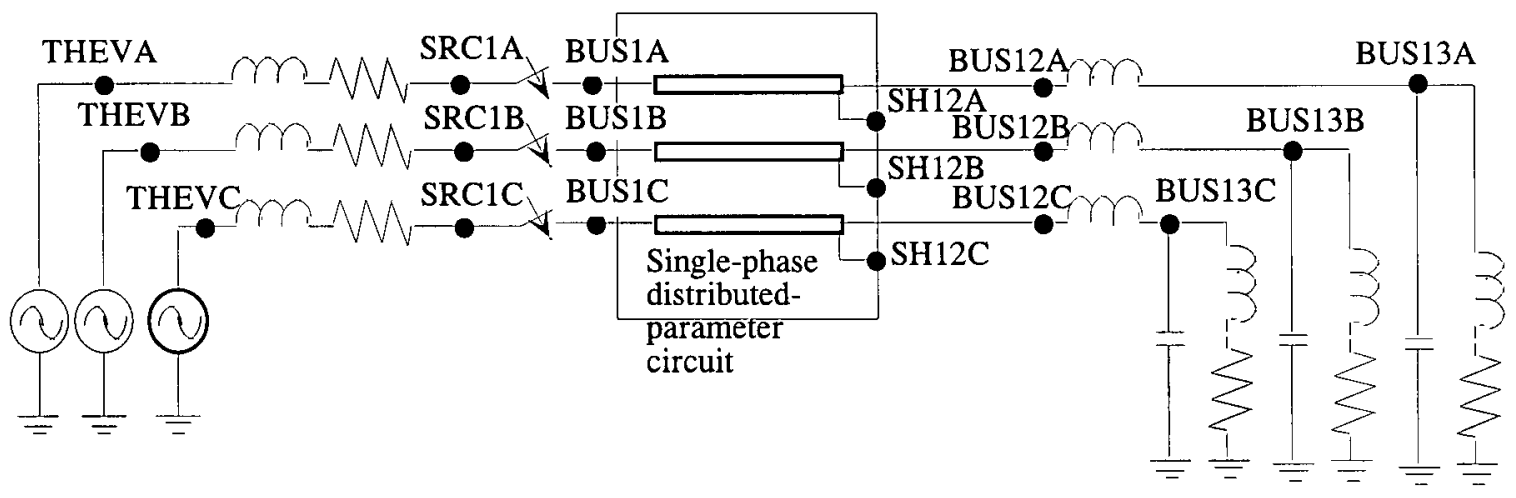

Figure A.6 Three-phase cable switching

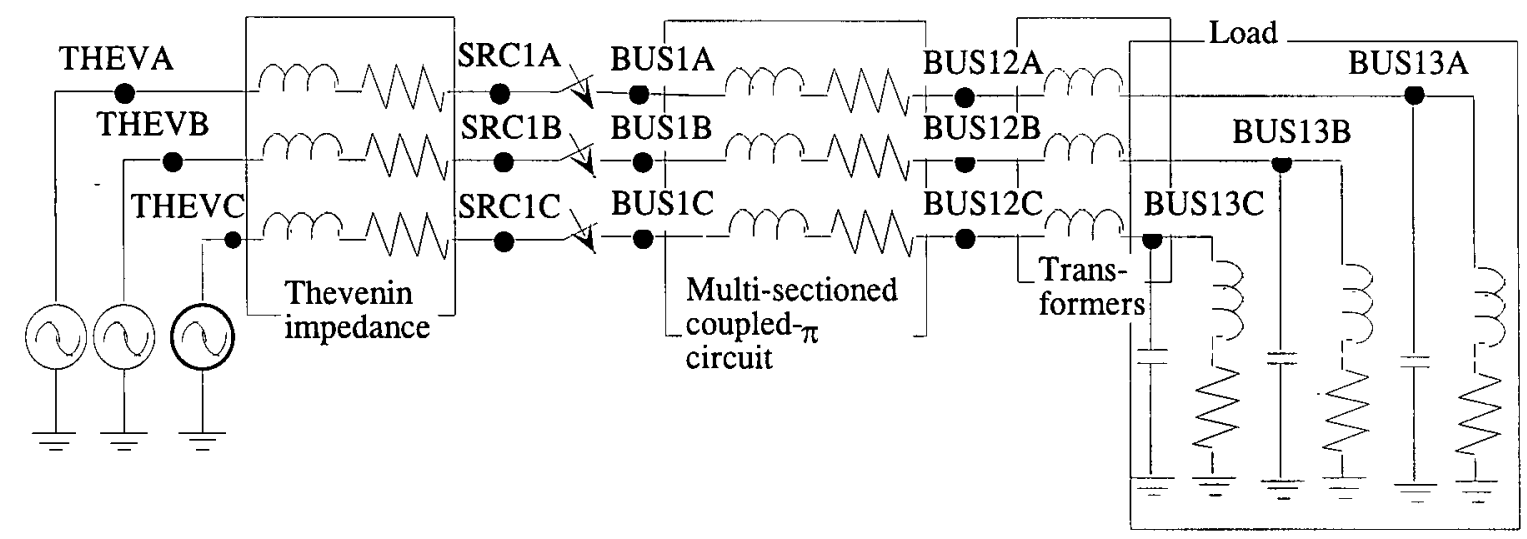

Figure A.7 Cable switching with crossbonding 


\section{A.1.4 Transformer Switching}

When an unloaded transformer is energized, there can result large and often distorted inrush current due to nonlinear inductance of transformer core. The major factor of concern are, therefore:

i) Current magnitude (typically, 2-5 times that of the rated current);

ii) Harmonic component (such as rectified DC wave).

The inrush current will vary with source voltage phase and remnant flux. The transformer switching sub-menu has the following cases:

\section{A.1.4.1 Transformer and Shunt Reactor Energization}

In this example shown in Figure A.8, the single-phase transformer with no load is considered. If a transformer is unloaded, the equivalent circuit will be reduced (from the T-circuit) to a single reactor (of the transformer core) as shown in the figure. The inrush current will vary with source voltage phase and remnant flux.

The modeling of transformer (core) may then involve the following properties:

i) Nonlinear reactance of the transformer core;

ii) Hysteretic curve;

iii) Remnant flux. 
The current vs. flux saturation curve inductance can be modeled by piece wise linear nonlinear inductance (Type-93).

The current vs. flux curve can be obtained from point-by-point voltage vs. current curve supplied by transformer manufacturer using CONVERT subroutine included in the EMTP.

[Further Remarks]

(a) Remnant flux is included in Type-93 piece-wise linear inductance as initial value.

(b) In a three-phase transformer (bank or three-phase core) the three-phase core should be such modeled as the sum of remnant flux being zero.

(c) A large resistance (typically $10 \times 6 \Omega$ ) across the nonlinear inductance is designed to serve as hysteresis. Similarly, a large inductance $(10 \times 20 H)$ may be used to model flux.

(d) There is a conversion program available for saturable transformer data which is present in other versions of EMTP.

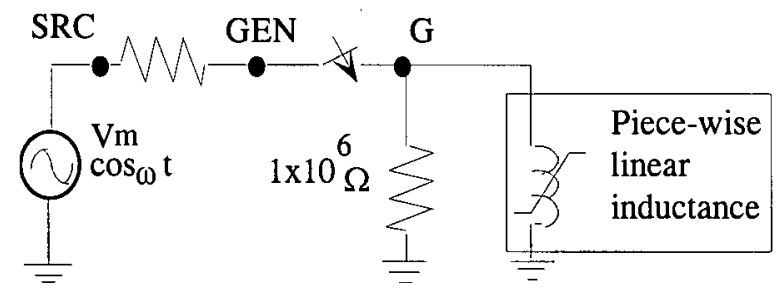

Figure A.8 Transformer switching 


\section{A.1.5 Load Energization}

The load energization sub-menu includes the following cases:

\section{A.1.5.1 Simple Switching (Single-Phase)}

This configuration shown in Figure A.9 represents an energization of a single-phase R-L load from an ideal source through a transmission line represented by a $\pi$-circuit. Single-phase representation of an originally three-phase circuit can be inaccurate because of the difference in voltage phases at the moment of CBs closing.

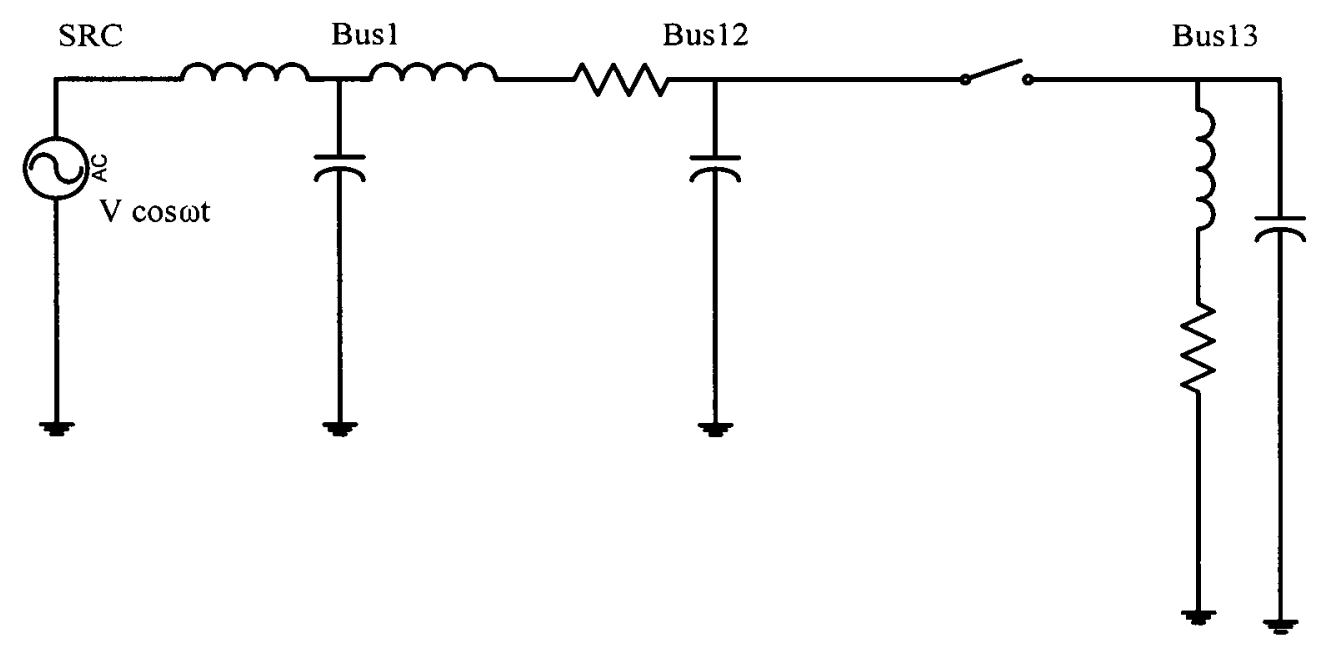

Figure A.9 Simple switching (single-phase)

\section{A.1.5.2 Simple Switching (Three-Phase)}

A short transmission line can be represented by coupled $\pi$-circuits as shown in Figure A.10. For fast transient solutions, however, distributed-parameter line models are generally better. You can use the $\pi$-models only when the traveling time is less than a reasonable time-step $\Delta \mathrm{t}$. 
The source voltage should be converted into the line to ground peak voltage. The three-phase R-L load can be calculated by the base kV/MVA (if their values are given in p.u.). Singlephase representation of an originally three-phase circuit can be inaccurate due to the difference in voltage phase of original phases at the moment of CBs closing. 

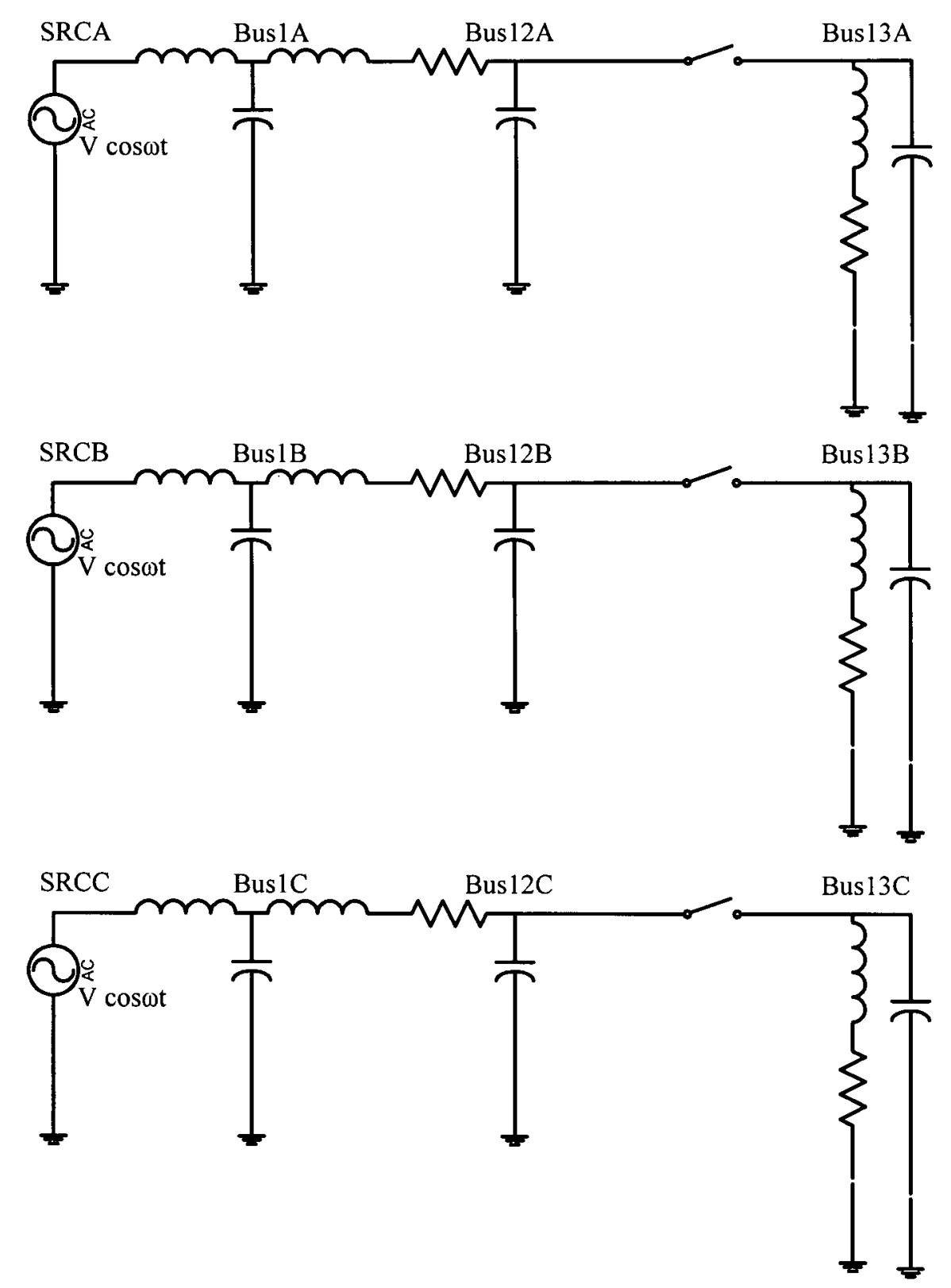

Figure A.10 Simple switching (three-phase) 


\section{A.2 Fault Analysis}

Modeling considerations for fault analysis are summarized as follows:

\section{(a) Source}

In most cases Thevinin equivalent of the source is used.

Simplification of voltage source into a Thevinin equivalent depends on the time-scale of simulation. Generally, such simplification is valid only for initial 2-3 cycles. For the analysis of longer time-scale, e.g. generator stability analysis, we need a detailed machine model.

\section{(b) Lines}

It is advisable to use a frequency-dependent model for an overhead line wherever data are available. Frequency-dependent model for underground cables is not available at present. For the frequency-dependent model input, detailed information of tower geometry is needed.

Using the REBUILD option in fdLine support program, we can approximate the tower geometry of a frequency-dependent line. The conductor geometry assumed by REBUILD option by fdLine is triangular. The height of conductors is given at the center of the triangle. Also, the program can guess the ground resistivity by default.

\section{(c) Fault}

Fault can be represented by a switch between a line and the ground with possible grounding resistance. To specify a fault location (distance from source, etc.), the user can split a line into 
two portions simply dividing by the distance.

\section{Further Remarks:}

Fault impedance is normally resistive but nonlinear (voltage-dependent) rather than timevariant. However, the tower footing resistance in lightening surge analysis can be better assumed as time-varying.

\section{A.2.1 Single-Line to-Ground of an Overhead Line}

In this example shown in Figure A.11 we consider a single-phase fault at the end of a long transmission line. The 120 mile transmission line is represented by a constant-parameter line model. The modeling does not include the frequency dependence of the line parameters.

\section{A.2.2 Single-Line to-Ground with Frequency Dependence}

The test system shown in Figure A.12 is used for BPA field test comparison. The source impedance is modeled by lumped inductance. The transmission line is represented by a frequency-dependent line. Support program fdLine is used to calculate equivalent tower geometry (separate data set is required for the fdLine option). 


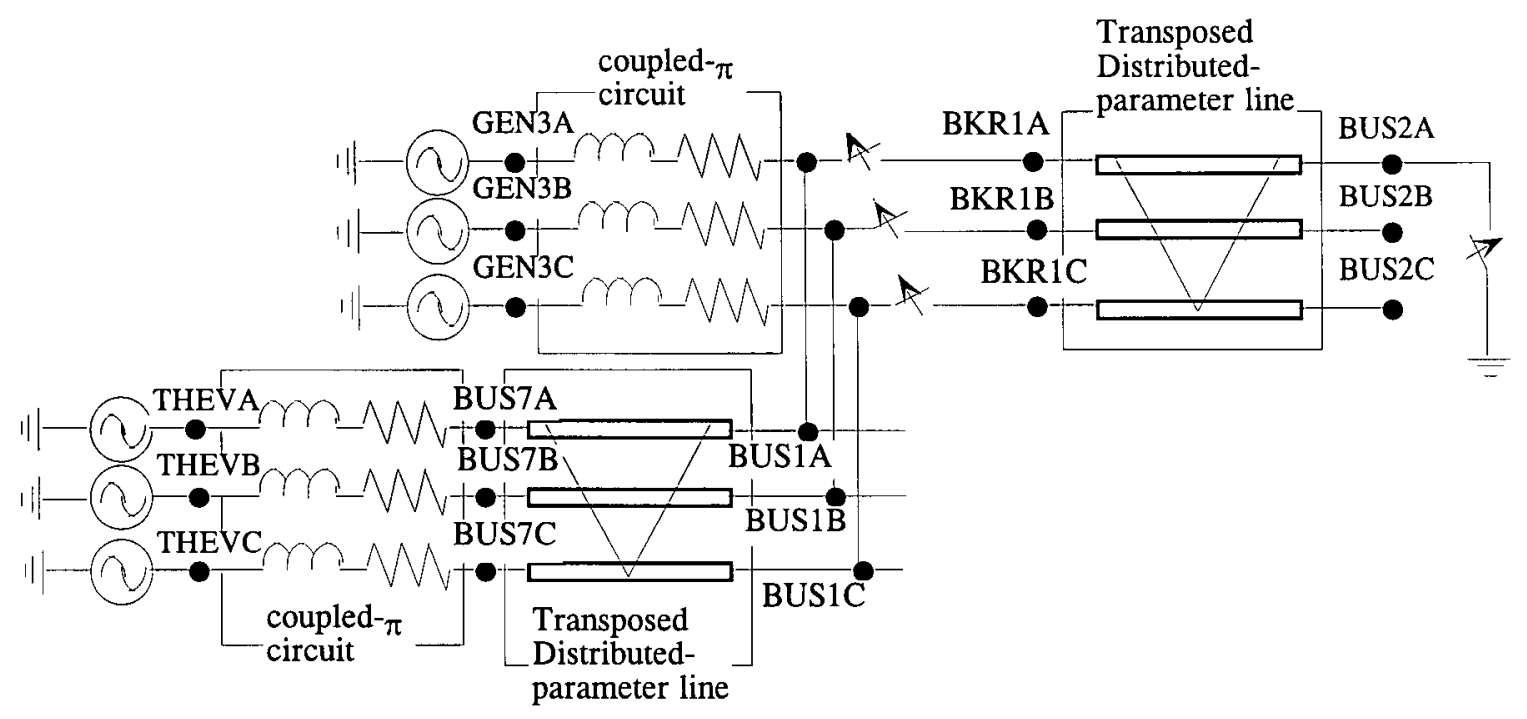

Figure A.1 1 Single-line to-ground fault

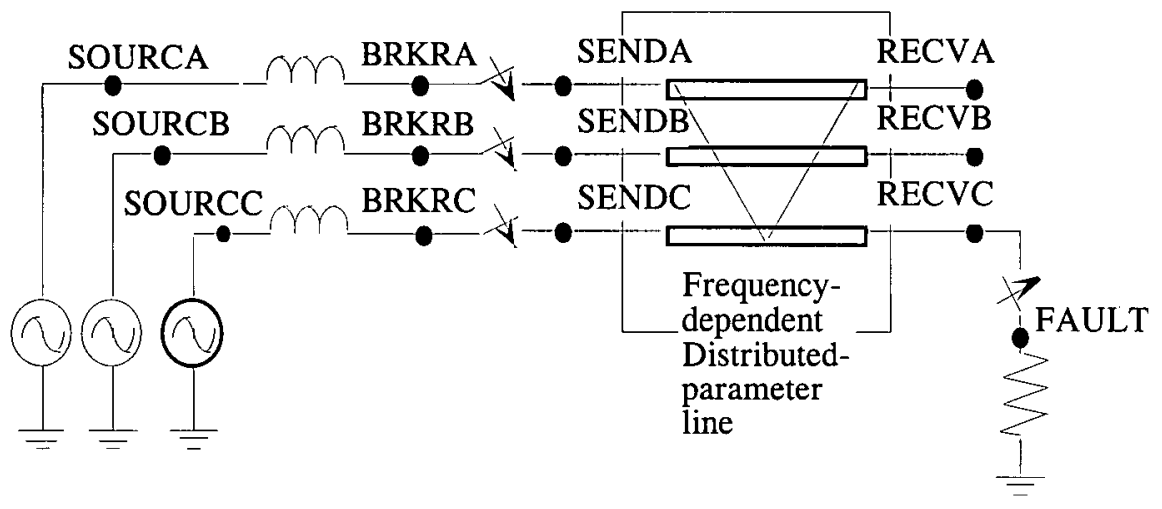

Figure A.12 Fault analysis with frequency dependence 


\section{A.2.3 Three-phase Short-Circuit for Transient Stability}

A three-phase to ground fault case is given in this example as shown in Figure A.13. The system configuration is given in the single-line diagram (but the actual data are three-phase). The fault is initiated at $\mathrm{t}=30 \mathrm{~ms}$, and CBs at Bus- 1 clears the faulted line after $100 \mathrm{~ms}$.

Line-to-ground fault and line-to-line short circuit are considered here.

The phenomena of concern may include:

i) Fault current on various paths from the source(s);

ii) Voltages during post-fault;

iii) Generator stability.

Modeling considerations include:

(a) For line-to-ground fault overhead lines should be modeled by frequency dependent model.

For short-circuit studies, overhead lines can be represented by constant parameter model. If in doubt, it is advisable to use frequency-dependent line model.

(b) Saturation of transformers should be included because the fault voltage is likely to produce harmonics. 


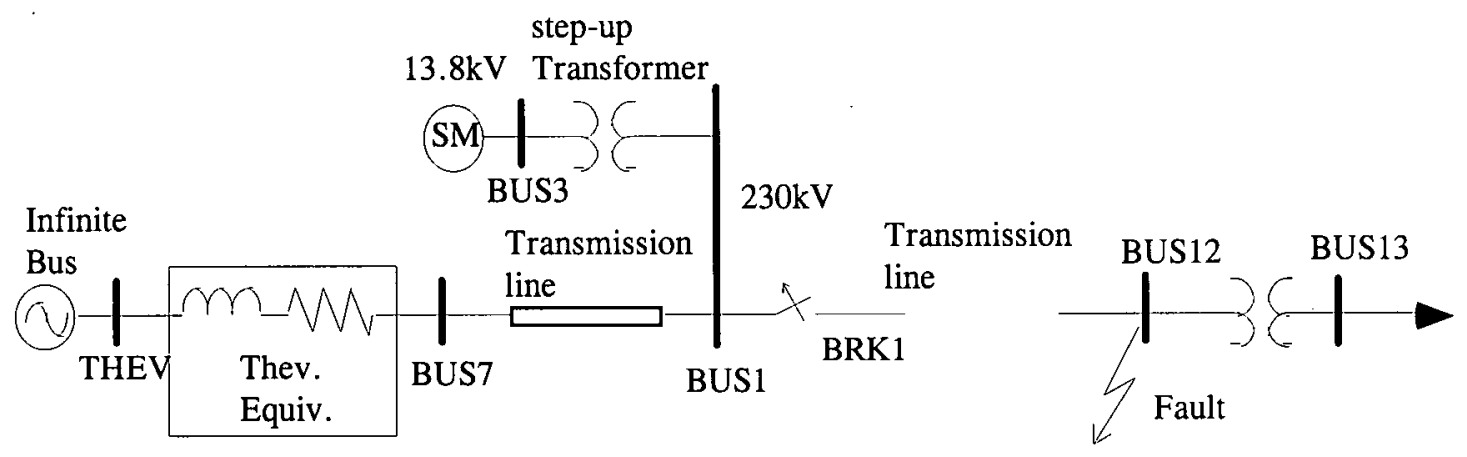

Figure A.13 Transient stability analysis

\section{A.2.4 Load Rejection}

The problem of load rejection often arises when radially connected generation is switched off at load end. The load rejection generally results in overvoltage by the following reasons:

i) Switching surge from a heavily loaded line to an unloaded line;

ii) The Ferranti effect (at the open end);

iii) Generator overspeeding.

The magnetic saturation in generator step-up transformer may result in ferroresonant oscillations. The overvoltage conditions can influence the lightening arrester selection.

In the sample circuit note the following modeling considerations:

- A synchronous machine model is used for a sinusoidal voltage source.

- If the switching surge and the Ferranti effect are only concerned the source can be modeled by a sinusoidal voltage source behind the subtransient reactance. 
- To represent the saturation of the transformer, a combination of ideal transformers and Type-93 nonlinear inductances are used for MicroTran.

- Some manual conversion of data may be needed for type-51 synchronous machines from Type-59 in other versions of EMTP.

The single-line diagram of a test system is shown in Figure A.14 (Actual data is three-phase). A synchronous machine model is used for a sinusoidal voltage source. The step-up transformer with saturation is represented by a combination of ideal transformers and Type-93 nonlinear inductances.

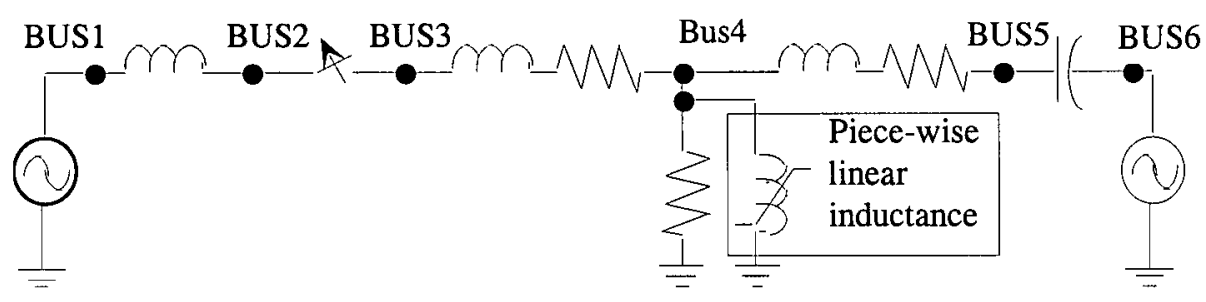

Figure A.14 Load rejection

\section{A.3 Ferro-resonance Analysis}

In this example shown in Figure A.15, a three-phase transformer bank is energized in only two phases and the ferro-resonance results from the interactions between the transformer and the capacitance among phases. A transformer is modeled by the T-equivalent with the core represented by nonlinear (piece-wise linear) inductance.

Ferro-resonance can occur when a nonlinear inductor resonates with a capacitor, typically 
when a high-voltage transmission line without load can act as a capacitance. Ferro-resonance may exhibit variety of distorted waveforms, typically sub-harmonic resonance.

A transformer is modeled by the T-equivalent with the core represented by nonlinear (piecewise linear) inductance as shown in the figure. The T-equivalent circuit is not valid for multiphase transformer cases because of the difference in positive- and zero-sequence impedances. At least the ideal transformer is needed in multi-phase cases. The support program BCTran can deal with such coupling effect.

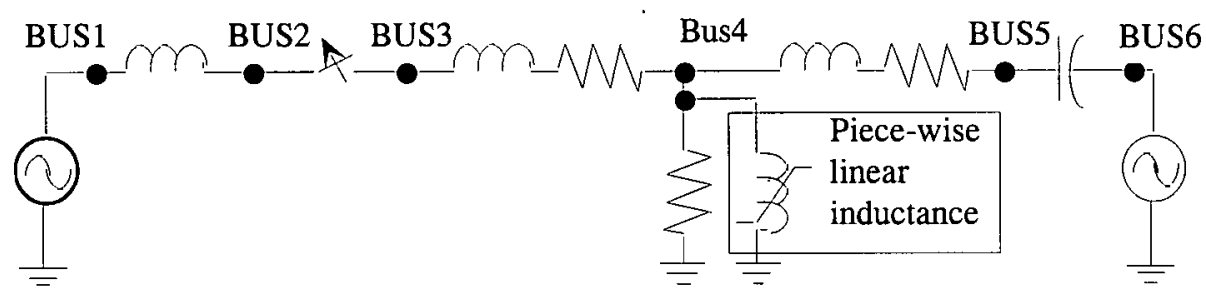

Figure A.15 Ferro-resonance analysis 


\title{
Appendix B
}

\section{EMTP Connec Subroutine for the New Line Model}

\author{
SUBROUTINE CONNEC ( IPHASE, ISTEP, ZTHEV, X, Y, NONLAD, NONLE, \\ 1 CURR, VOLD, VOPEN, VNONL, ILAST1, VZERO, T , DELTA2, IWHERE, ICHECK, ITMAX, \\ $2 *)$ \\ IMPLICIT NONE \\ C PROCEDURE ARGUMENTS : \\ INTEGER * 4 IPHASE , ISTEP , NONLAD, NONLE , ILAST1, IWHERE, ICHECK, ITMAX \\ REAL * $8 \quad Z T H E V, X, Y, C U R R, V O L D, V O P E N$, VNONL, VZERO , T , DELTA2 \\ C INTERNAL VARIABLES: \\ INTEGER * 4 I, K, L, DEN, MEM, MAXMEM, CNT \\ REAL $\star 8 \quad$ ZC, TAU, DT , A, FACT , RMAT, ZMAT, HIST, HVEC (IPHASE) \\ PARAMETER (MAXMEM=30) \\ DIMENSION ZTHEV $(*), X(*), Y(*), \operatorname{NONLAD}(*), \operatorname{NONLE}(*), \operatorname{ILAST} 1(*), \operatorname{CURR}(*)$, \\ $+\quad \operatorname{VOLD}(*), \operatorname{VOPEN}(*), \operatorname{VNONL}(*), \operatorname{VZERO}(*), \operatorname{RMAT}(3), \operatorname{ZMAT}(3)$, \\ $+\quad \operatorname{HIST}(2, \operatorname{MAXMEM})$
}


SAVE CNT , MEM, HIST

C

C CONNEC

C

C

C

c

C

C

C

C

C

C

C

C

C

C

C

C

C

C

C

C

C

C

$\mathrm{C}$

C

With this routine we hope to connect another version of a line model to MICROTRAN. While other line models require that the time step DT be less than or equal the traveling time TAU, the new model tries to overcome this limitation. The version implemented herein can only handle single phase lines. Purpose of this implementation is to gain more insight of the behavior and characteristics of this model.

A description of the subroutine arguments only includes those arguments that are actually used. For more details consult the MICROTRAN Manual (9/1992, Section 8.6) or Dr. H.W. Dommel. Following is a description of variables used in this subroutine:

A ratio of time step DT minus traveling time TAU and the time step DT:

$$
\begin{array}{lll}
A=(D T-T A U) / D T & \text { for } & D T>T A U \\
A=0.0 & \text { for } & D T<=\text { TAU }
\end{array}
$$

AUX1 auxiliary variable.

AUX2 auxiliary variable.

AUX3 auxiliary variable.

AUX4 auxiliary variable.

CNT index counter for history memory HIST (ring storage). Every time it reaches MEM, it resets itself to one.

CURR a vector containing the currents of the previous time 
C

C

C

OUTPUT) .

C

C

C

C

C

C

C

C

C

C

C

C

C

C

C

C

C

C

C

C

C

C

C

C step on input. This vector is modified at the end of this procedure to contain the new currents of the current time step on output (SUBROUTINE INPUT \& DELTA2 Half the integration time step DT (SUBROUTINE INPUT). DT the current time step.

FACT a factor for notational convenience.

HIST internal memory for history values. Especially needed if $\mathrm{DT}<\mathrm{TAU}$. At each time step, the history values are calculated from past voltages and current and stored in this memory. After a time span TAU they are called from the memory.

HK, HM history current sources at nodes $\mathrm{K}$ and $\mathrm{M}$.

I auxiliary variable.

IKM, IMK currents of the previous time step at nodes $K$ (flowing from $\mathrm{K}$ to $\mathrm{M}$ ) and $\mathrm{M}$ (flowing in the opposite direction). K auxiliary variable.

MAXMEM maximum possible number of memorable history values. MEM number of used memory MEM $<=$ MAXMEM.

RMAT matrix containing the line impedance. Hence, it is the inverse of the line admittance matrix. Its storage scheme is explained under ZTHEV.

VK, VM voltages of the previous time step at nodes $K$ and $M$.

VOLD a vector containing the voltages of the previous time step (SUBROUTINE INPUT).

VOPEN a vector containing the open circuit voltages of the external network (SUBROUTINE INPUT). $X, Y$ parameter vector. $X(1)$ contains the characteristic 


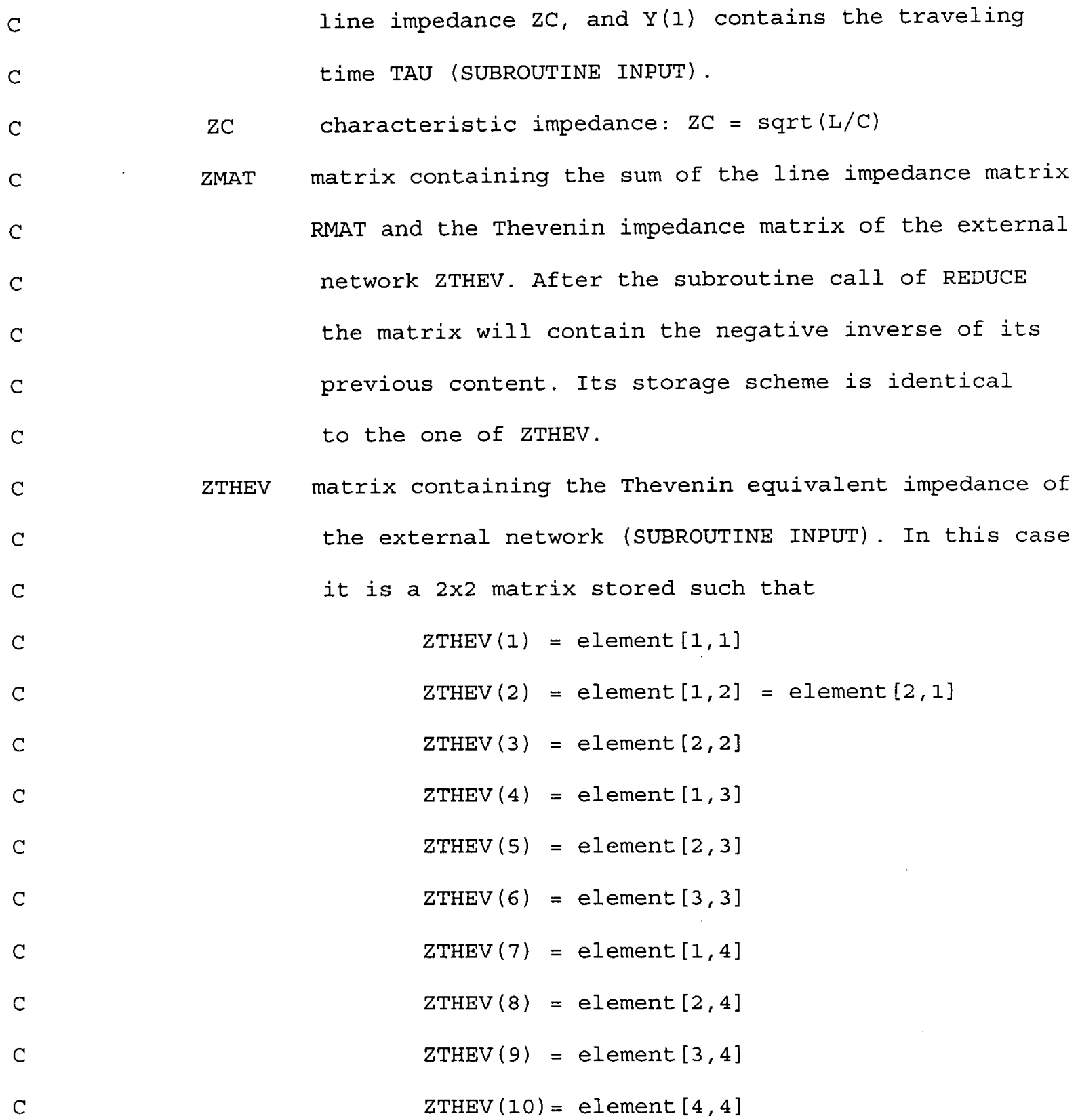

$\mathrm{zC}=\mathrm{X}(1)$

$\mathrm{TAU}=\mathrm{Y}(1)$

$\mathrm{DT}=2$. ODO $* \mathrm{DELTA} 2$

$\mathrm{A}=0.0 \mathrm{DO}$ 
IF (DT.GT.TAU) $A=(D T-T A U) / D T$

$\mathrm{FACT}=\mathrm{ZC} /(\mathrm{A} \star \star 2-1 . \mathrm{OD} 0)$

C Initialization of history memory (zero initial conditions only):

IF (ISTEP.EQ.I) THEN

MEM=1+INT $($ TAU/DT)

$\mathrm{CNT}=0$

IF (MEM.GT.MAXMEM) THEN

PRINT*, "CONNEC: Not enough history memory."

PRINT*, " Use a bigger time step."

STOP "PROGRAM TERMINATED"

END IF

DO $I=1, M E M$

DO $\mathrm{K}=1$, IPHASE

$\operatorname{HIST}(K, I)=0.0 \mathrm{DO}$

END DO

END DO

END IF

C RMAT is the inverse admittance matrix:

$\operatorname{RMAT}(1)=-(\mathrm{A} * \star 2+1.0 \mathrm{D} 0) *$ FACT

$\operatorname{RMAT}(2)=-2.0 \mathrm{DO} * \mathrm{~A} * \mathrm{FACT}$

$\operatorname{RMAT}(3)=\operatorname{RMAT}(1)$

C We now build the combined impedance ZMAT=RMAT+ZTHEVenin:

DO I $=1,($ IPHASE $\star * 2+I P H A S E) / 2$

$\operatorname{ZMAT}(I)=\operatorname{ZTHEV}(I)$

END DO

$\operatorname{ZMAT}(1)=\operatorname{RMAT}(1)+\operatorname{ZMAT}(1)$

$\operatorname{ZMAT}(2)=\operatorname{RMAT}(2)+\operatorname{ZMAT}(2)$ 


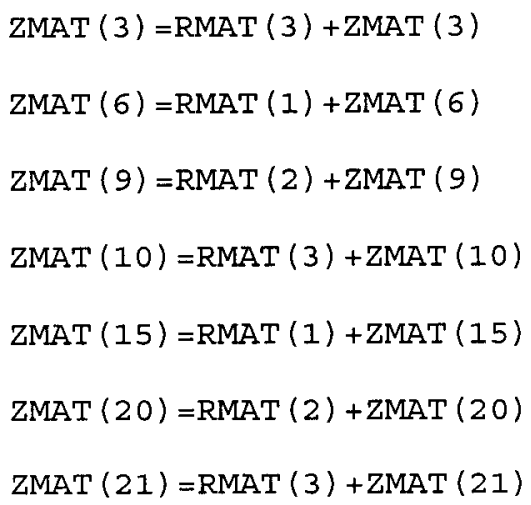




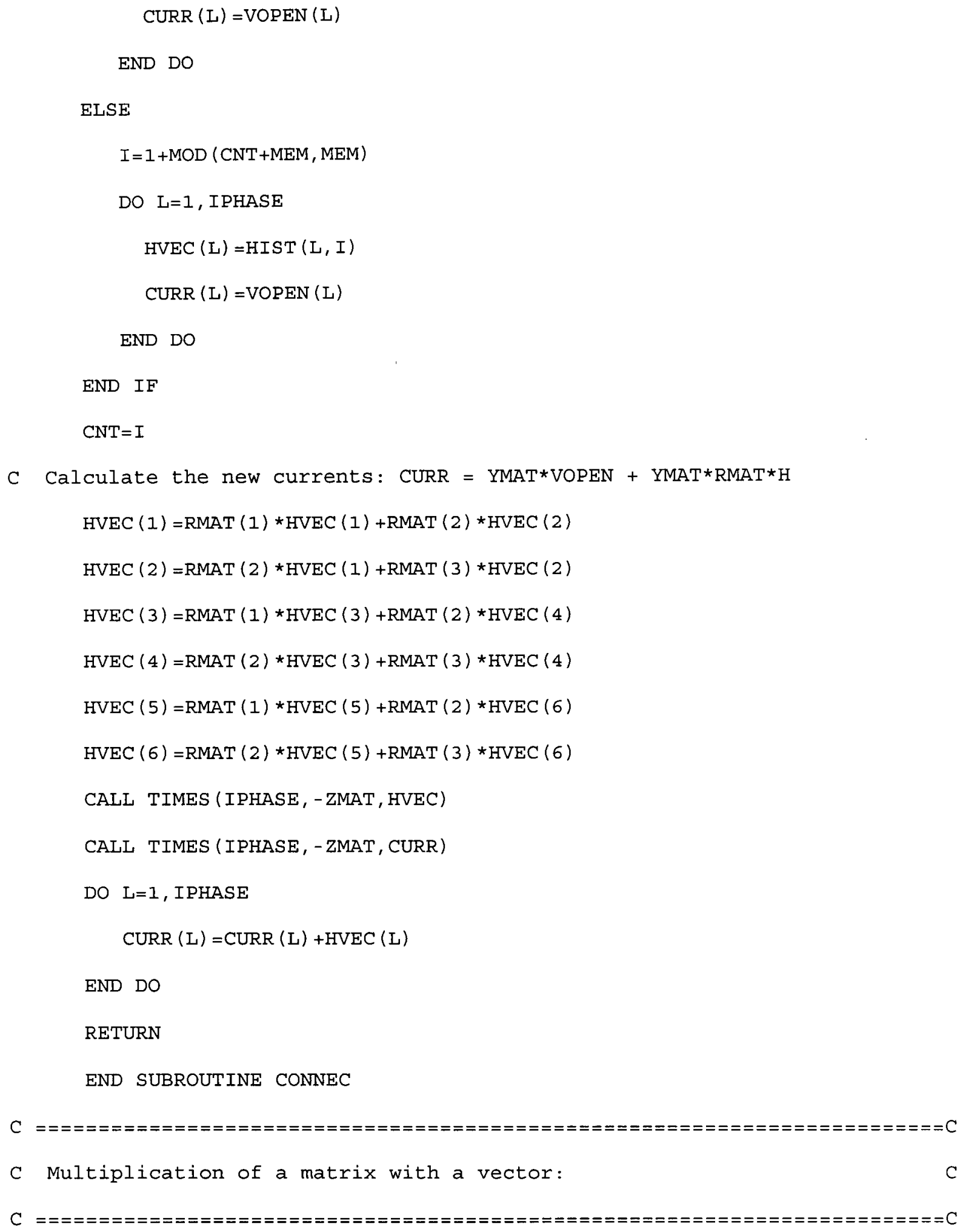




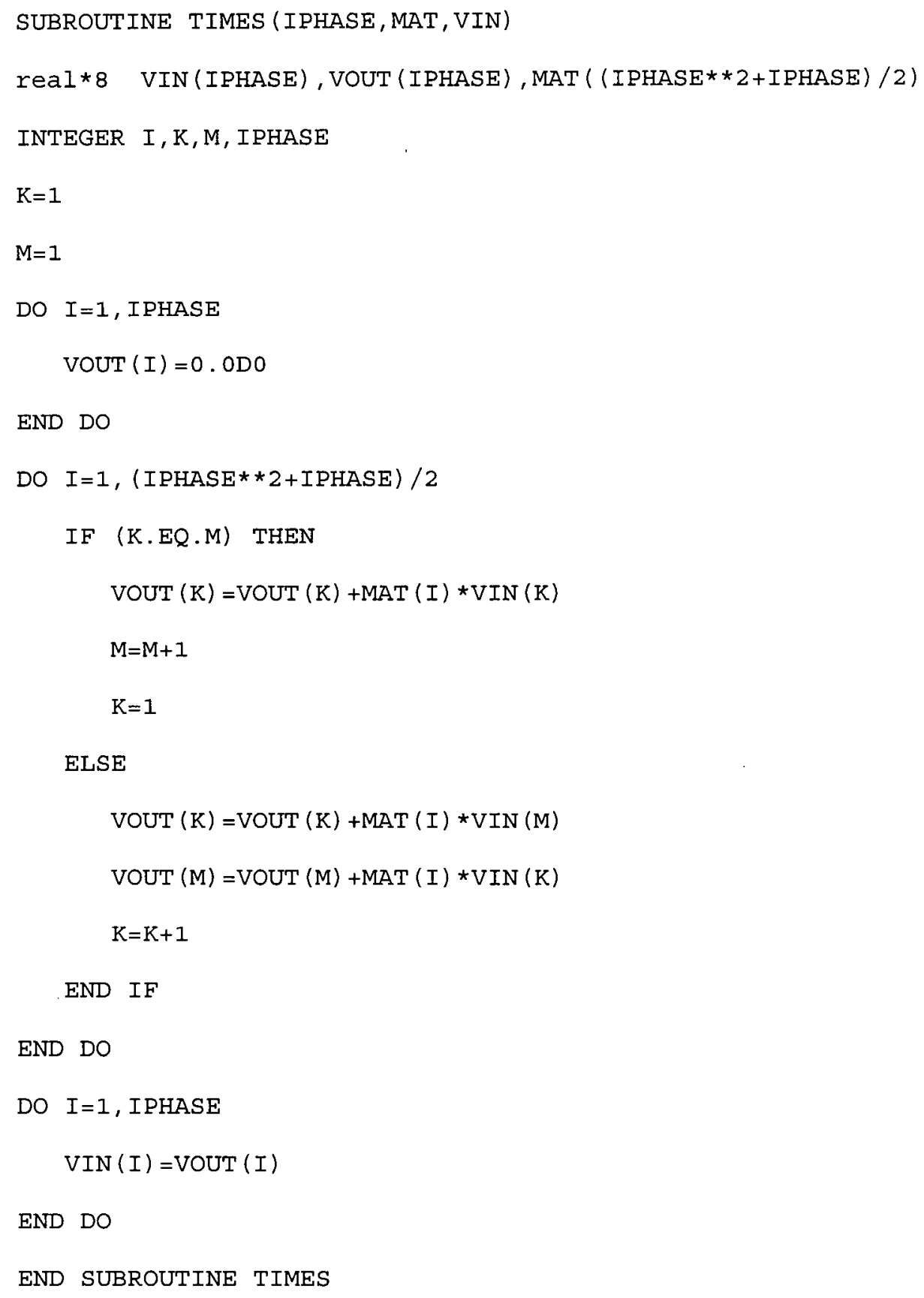




\section{Appendix C}

\section{Interpolation Error Analysis}

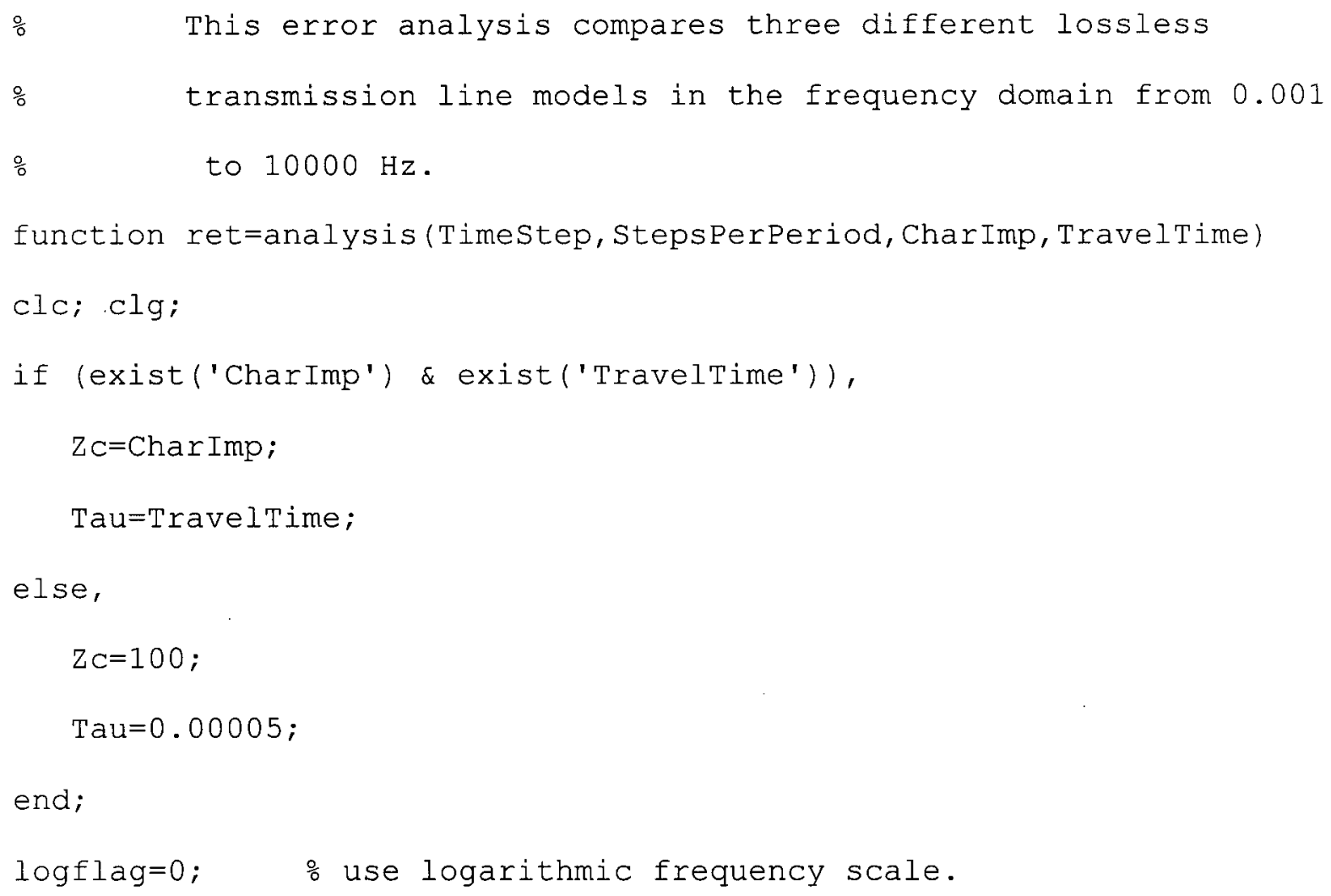


fprintf(1,'HARMONIC ANALYSIS OF LOSSLESS TRANSMISSION LINE MODELS $\left(n^{\prime}\right)$;

ret $=1$;

$j=\operatorname{sqrt}(-1) ;$

ToDeg=180/pi;

Huge $=1.0 \mathrm{E} 20$

StepsPerDecade $=50$;

$\operatorname{fmin}=100 . ;$

$f \max =10000.0$

$\mathrm{d} \log f=1 /$ StepsPerDecade;

decades $=\log 10($ fmax $)-\log 10($ fmin $)$;

$d f=(f \max -f m i n) /\left(1+\right.$ decades ${ }^{*}$ StepsPerDecade $)$;

$\circ *$

$\star \star$

응

o $\star \star$

$\mathrm{k}=0$ ；

$f=f \min ;$

while $(f>=f \min \& f<=f \max )$, $\mathrm{w}=2 * \mathrm{pi} * \mathrm{f} ;$

$\operatorname{Ad}(1,1)=\cos (w * T a u)$;

$\operatorname{Ad}(1,2)=-1$;

$\operatorname{Ad}(2,1)=-1$;

$\operatorname{Ad}(2,2)=\cos \left(w^{\star}\right.$ Tau $) ;$ 


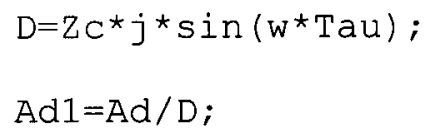

\% Open Circuit Gain, Short-Circuit Susceptance and Reactance

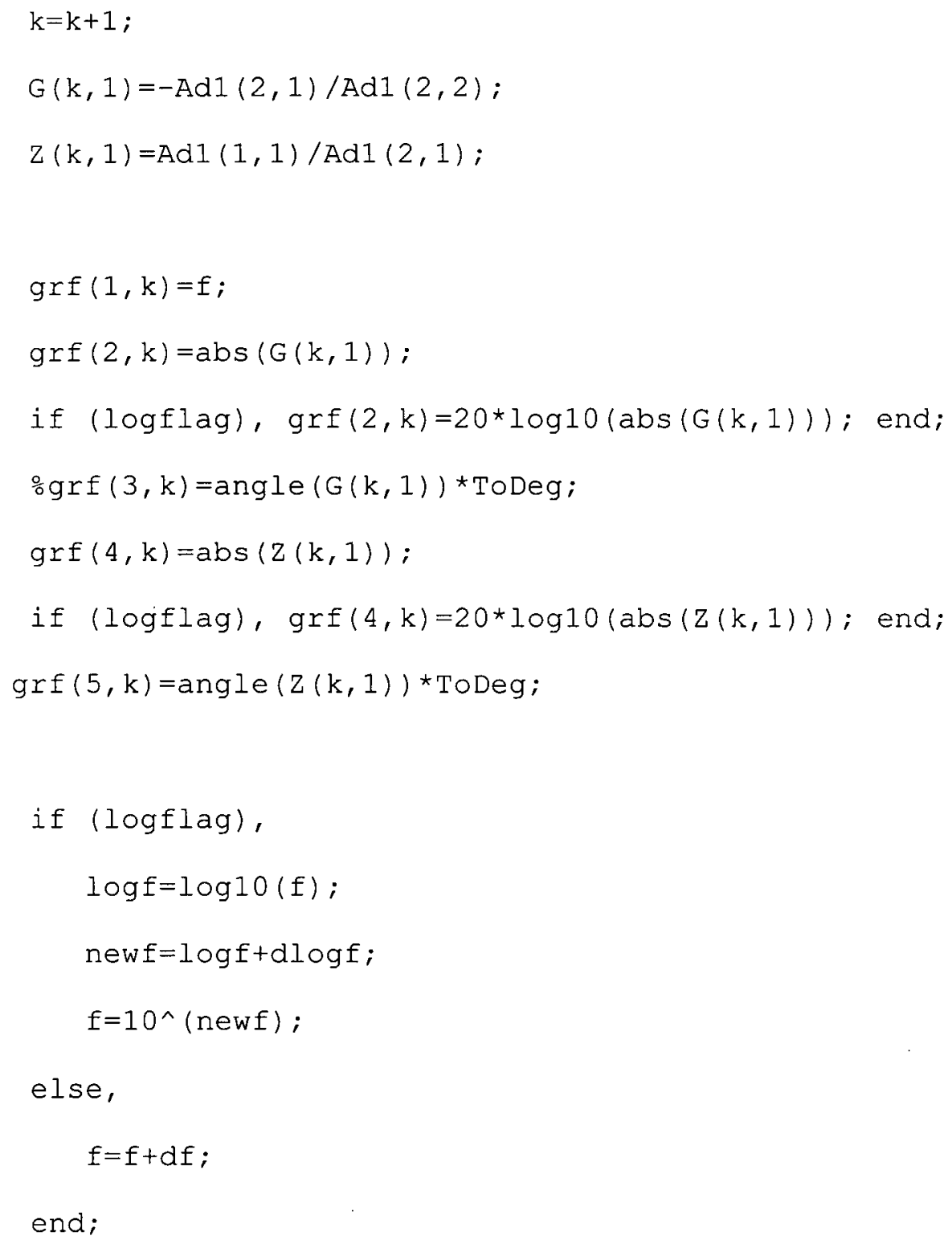




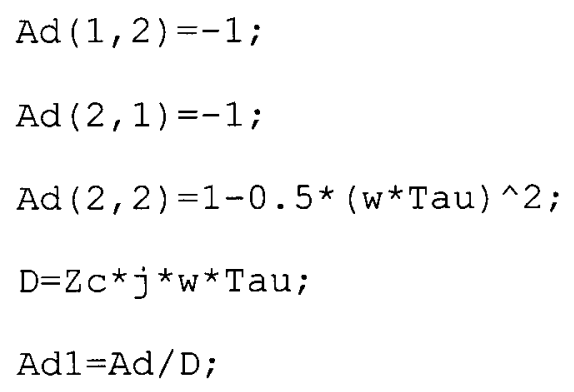

\% Open Circuit Gain, Short-Circuit Susceptance and Reactance

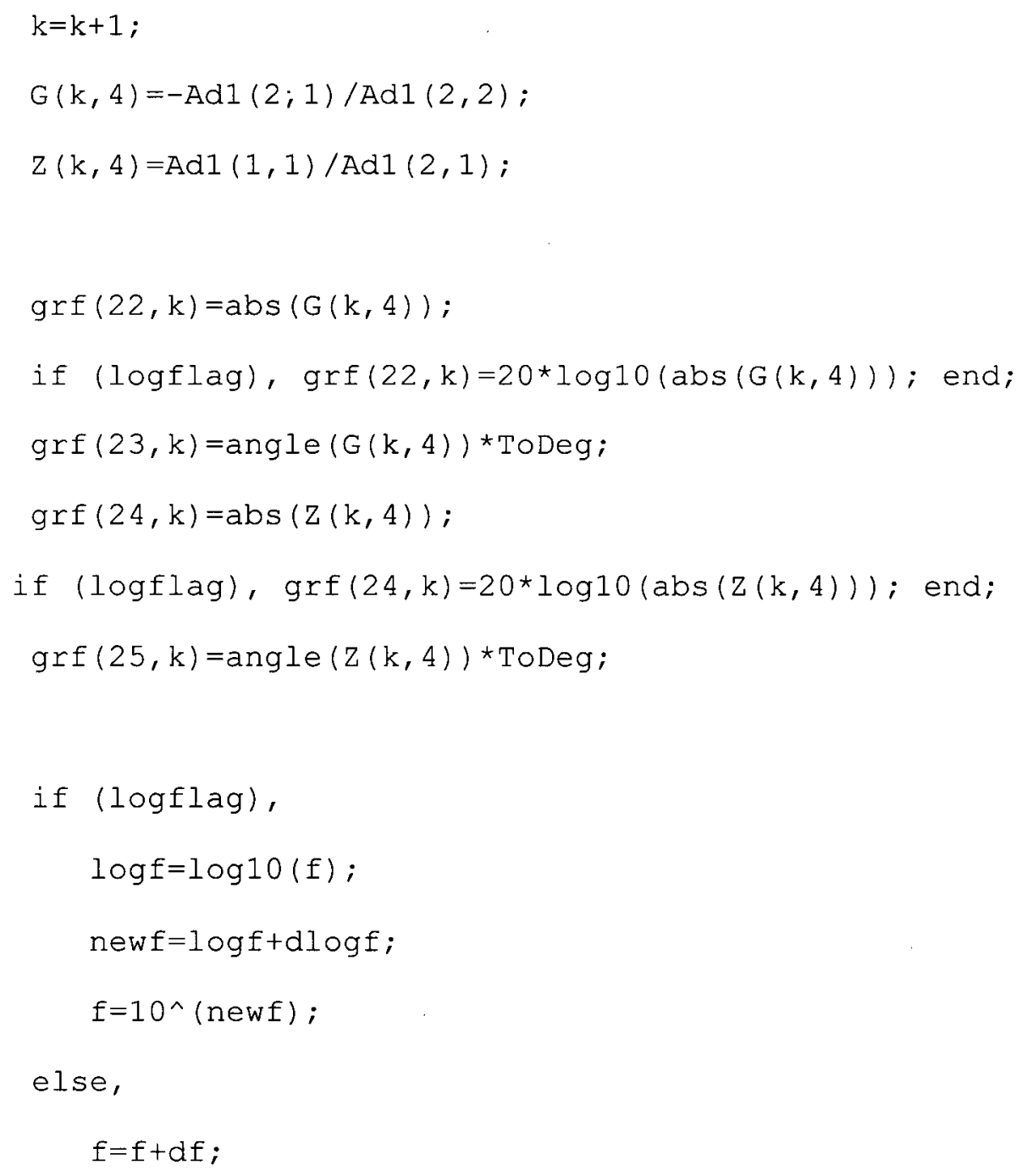


end;

end;

odisp('Done. Press any key to continue.'); pause;

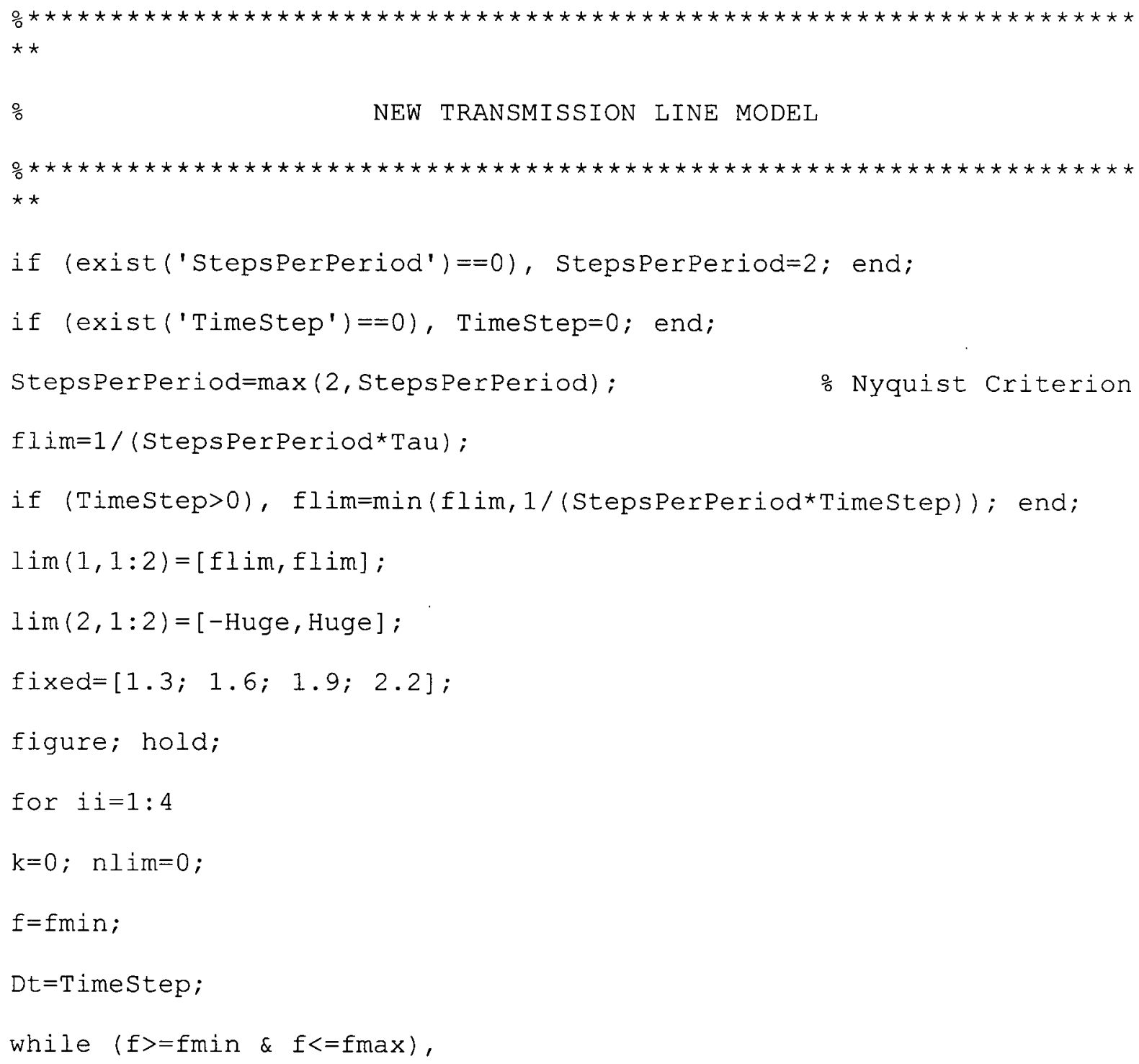




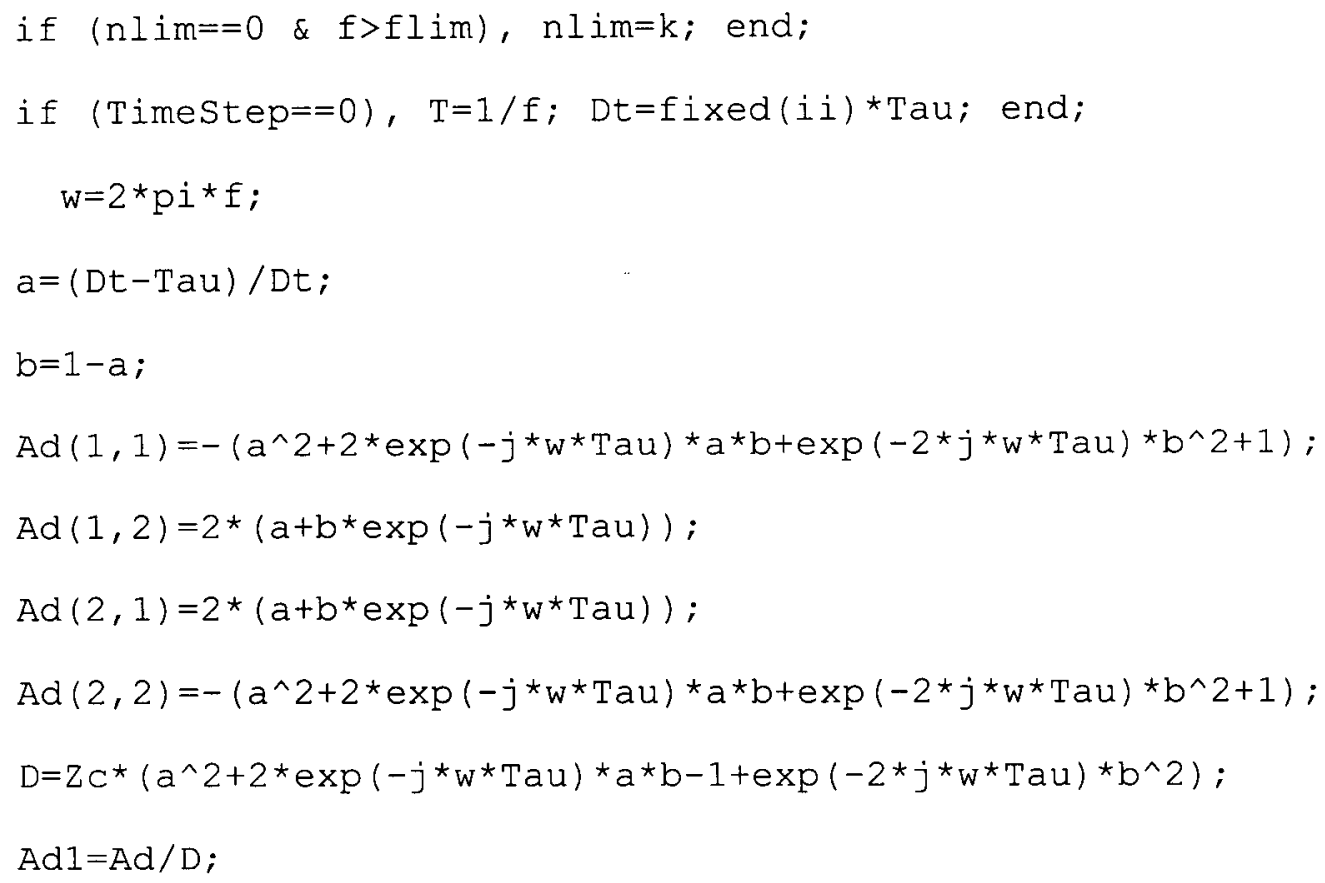

\% Absolute Deviation: 


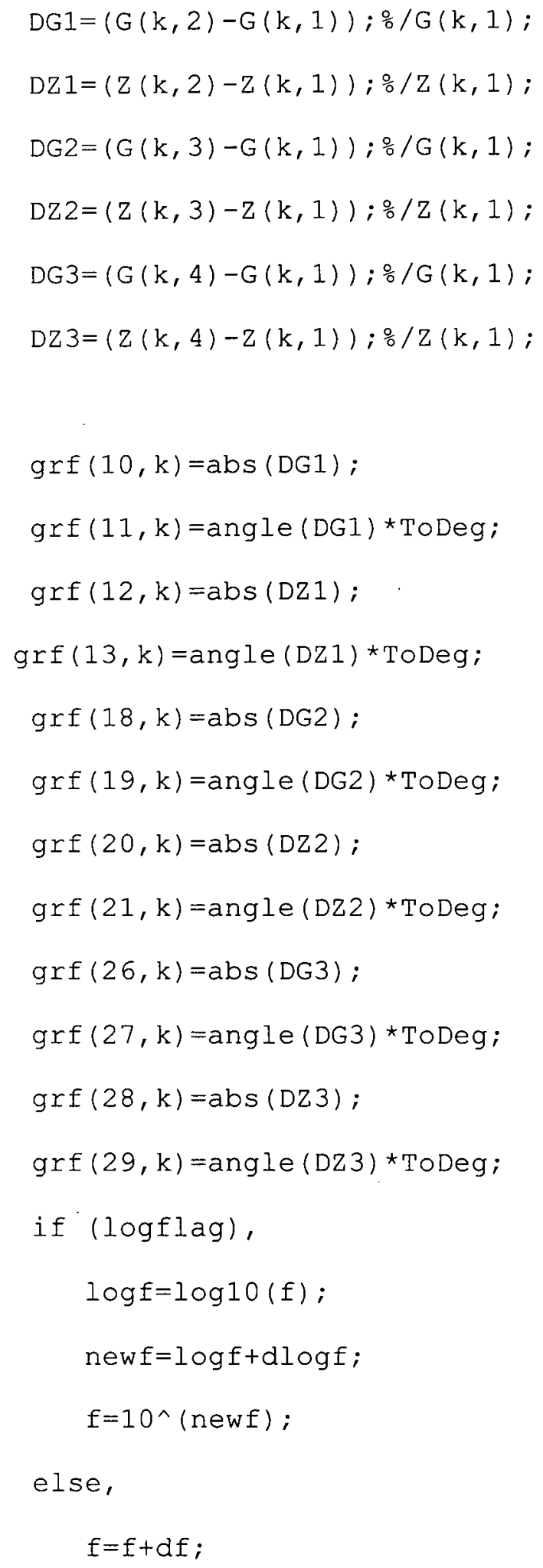




\section{end;}

end;

if (nlim==0 \& flim>=fmax), nlim=k; end; nlim=max (nlim,l);

fprintf $\left(1, ' \backslash n\right.$ Press any key to continue. $\left.\backslash n^{\prime}\right)$;

pause;

fprintf $\left(1, ' \backslash n\right.$ Press any key to continue. $\left.\backslash \mathrm{n}^{\prime}\right)$;

pause;

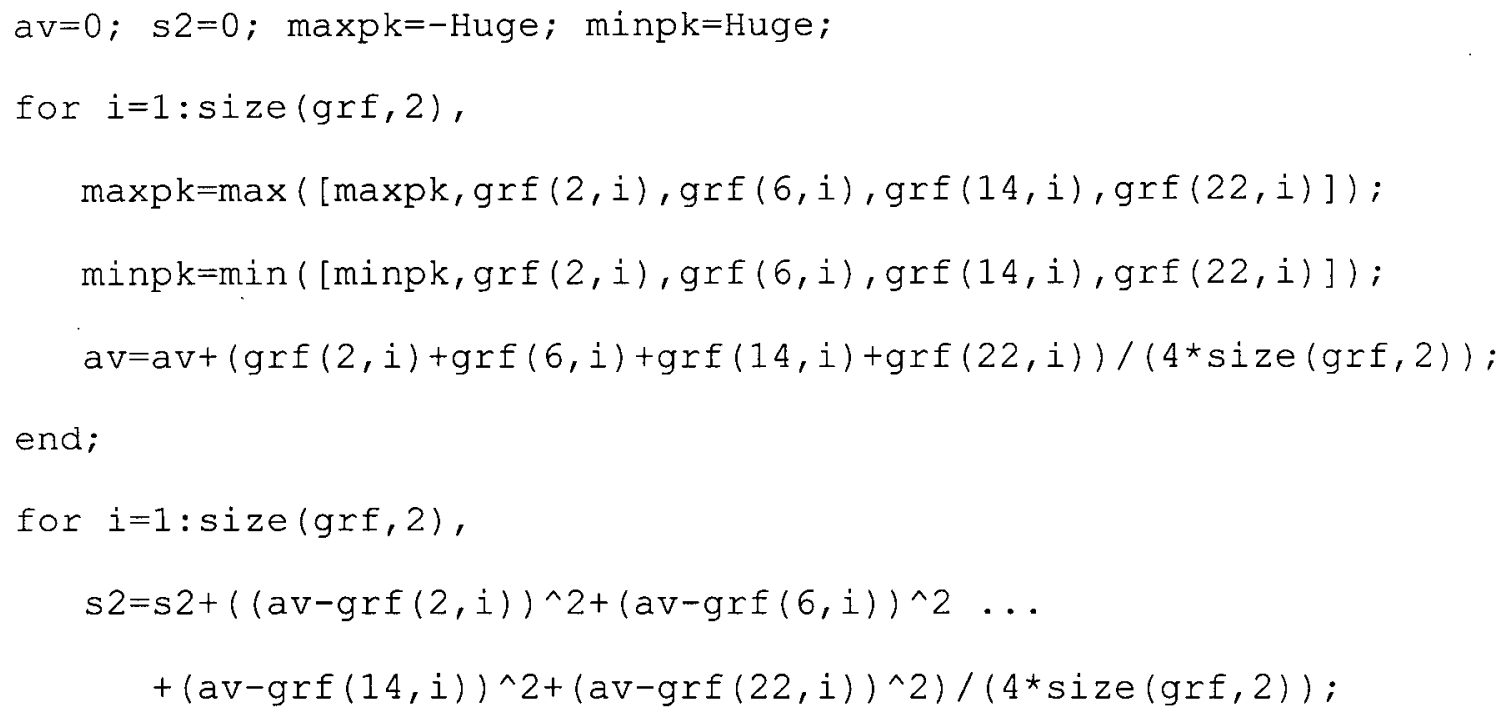

end;

$s=\operatorname{sqrt}(s 2)$

$\mathrm{up}=\mathrm{av}+\mathrm{s} ;$

$10=a v-s ;$

figure (1);

subplot $(2,1,1)$;

semilogx (grf $(1,:), \operatorname{grf}(2,:), y^{-1}, \ldots$ 


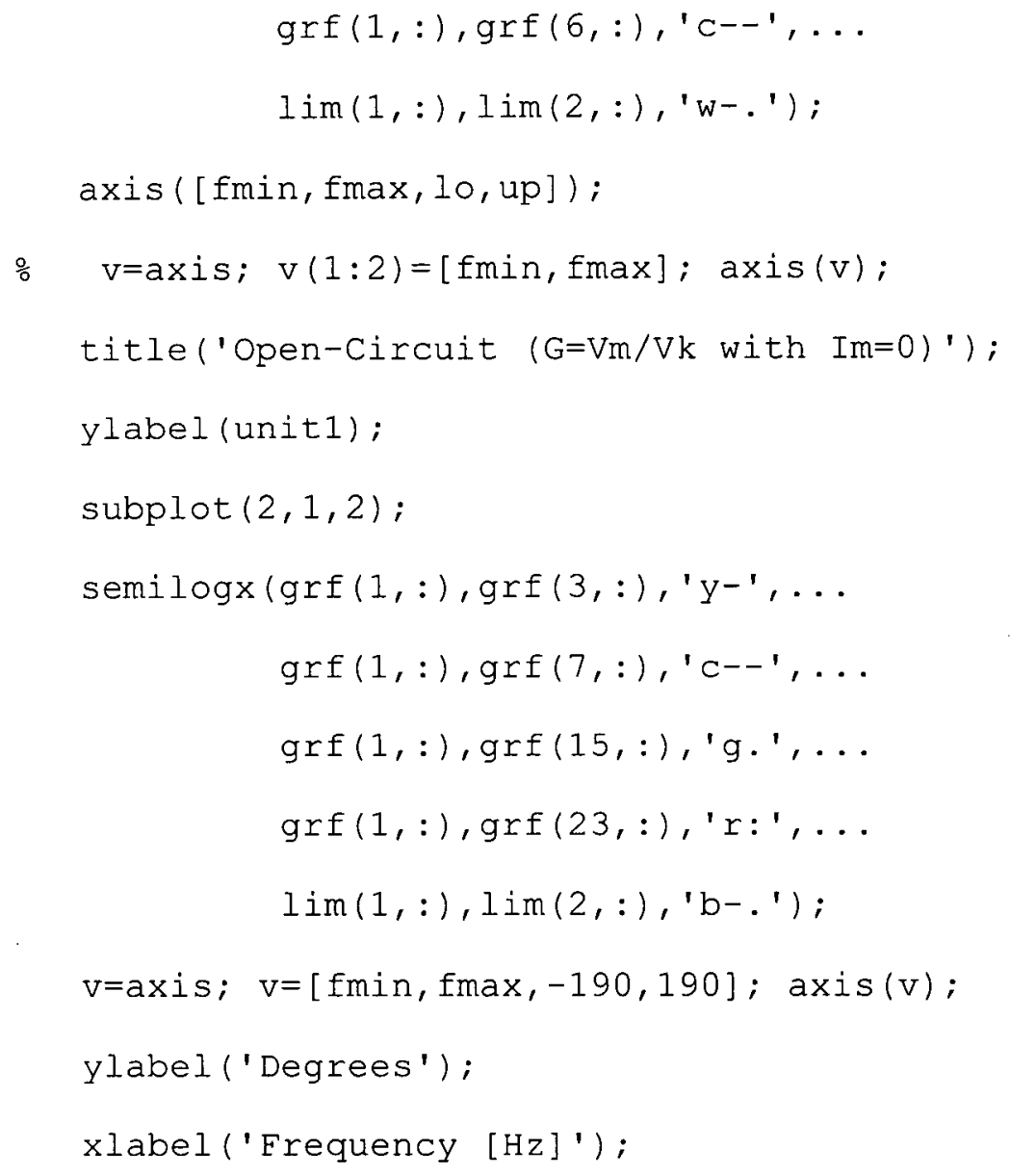




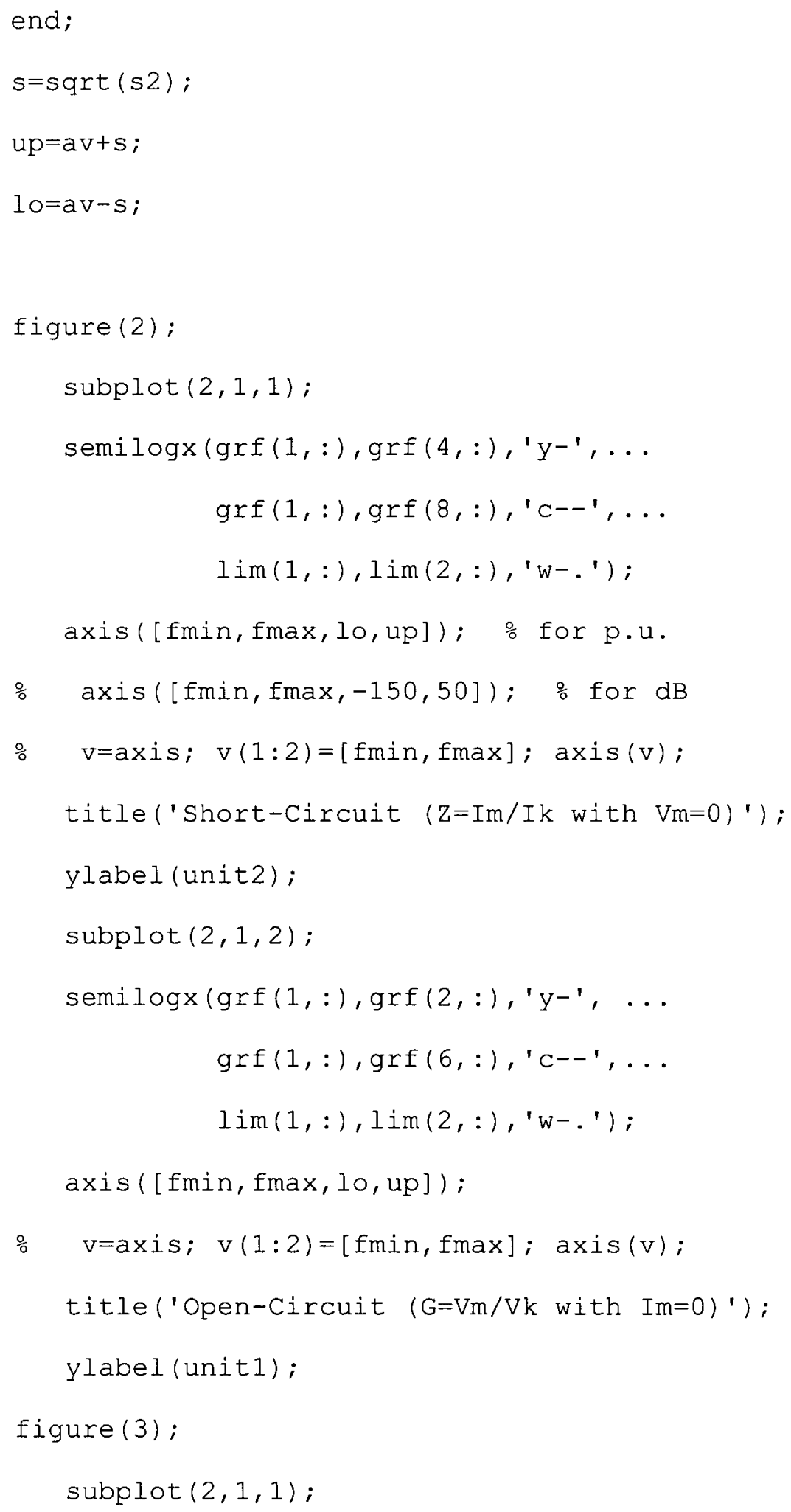




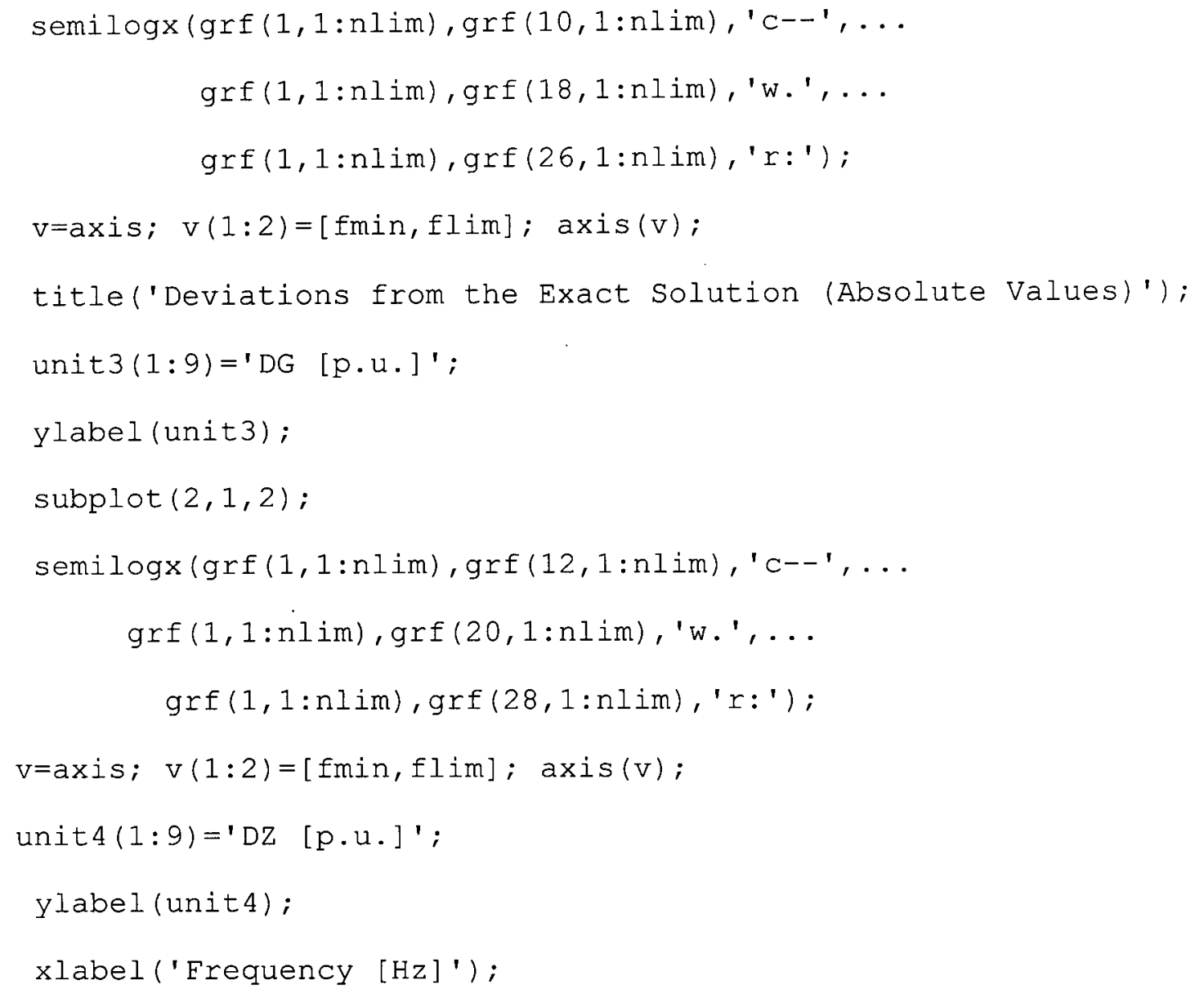




\section{Appendix D}

\section{Input \& Output Data Files for EMTP}

Table D.1:Data input file for the switching surge case study

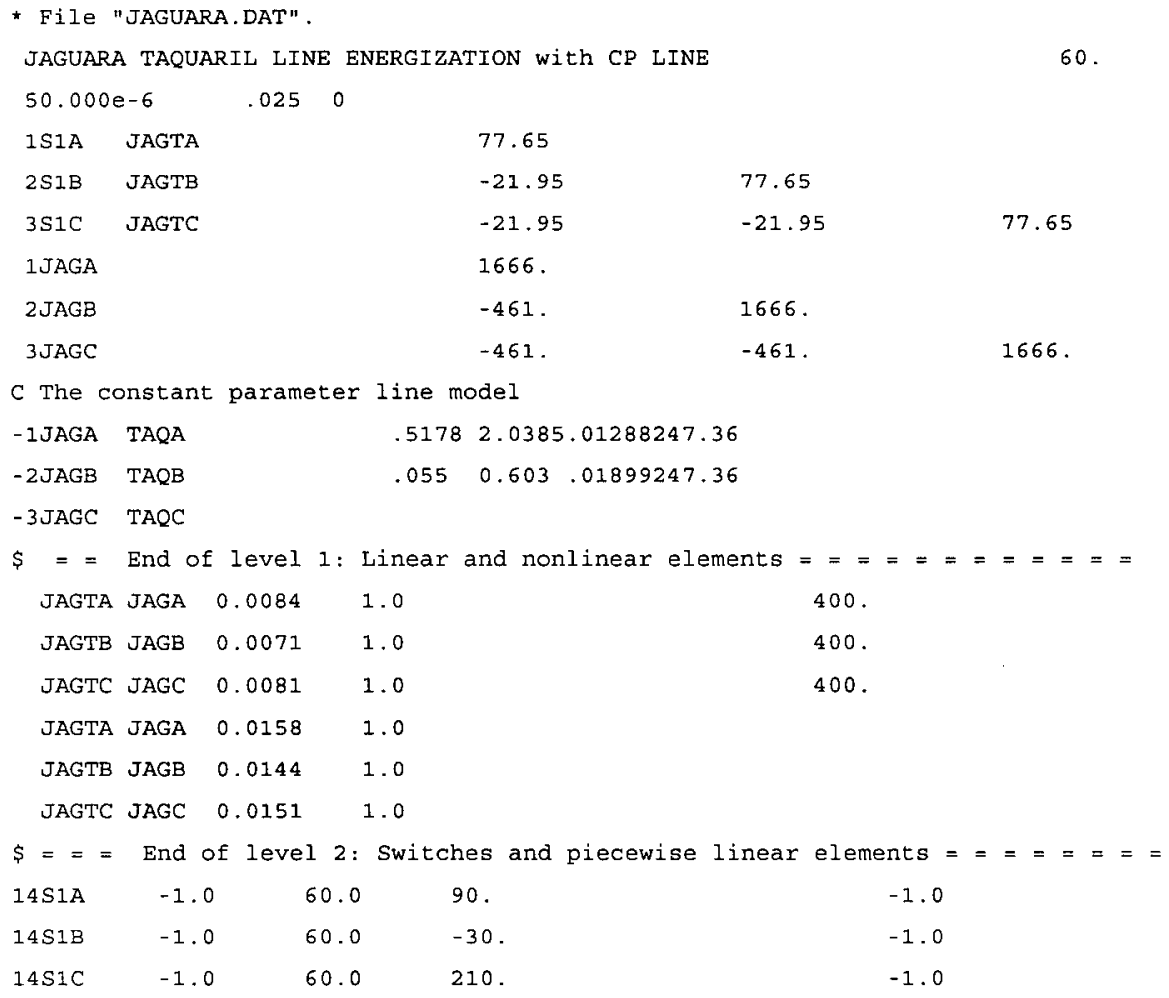


Table D.2:Output file for switching surge case study

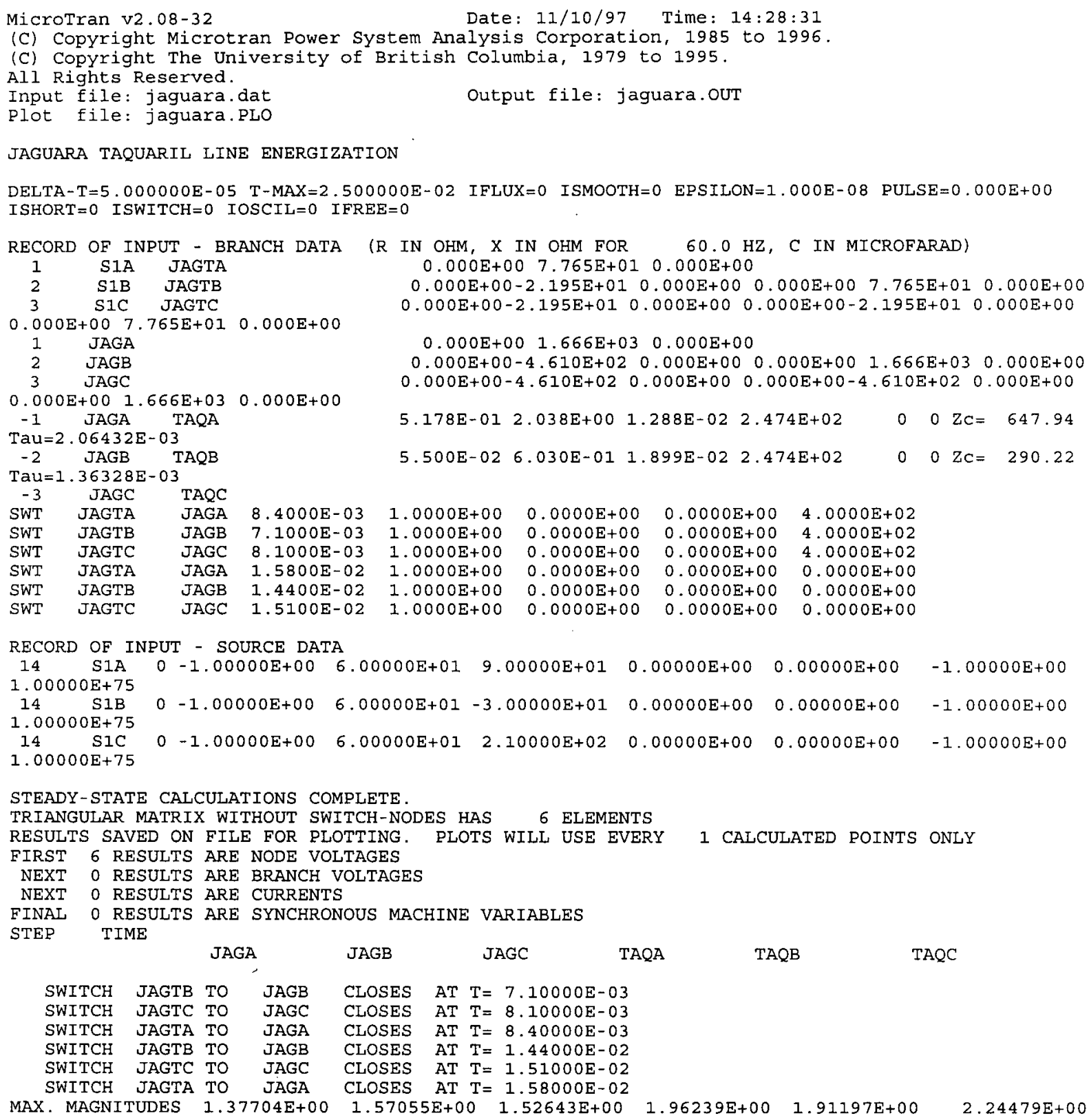


Table D.3:The fd Data input file for Jaguara case study

* FD DATA FIle for JAGUARA CASE

line-model

METRIC

$\begin{array}{llll}1.3333 .066998 & 4 \\ 2.3333 .066998 & 4 & \\ 3.3333 .066998 & 4 & \\ 0.5 & 4.188 & 4 & 50 \\ 0.5 & 4.188 & 4 & 50\end{array}$

100. 1000 .

*

* .ctlfit 15-1-100

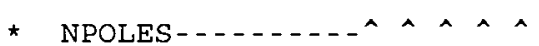

* IQUICK-......--1 | $\mid$

* IDYNAM-........- | |

* IALL -........-

* ICALC -.......

.outfit
BALANCED

$$
30.38
$$

$-8.5$

$22.91 \quad 8.31$

22.918 .31

22.918 .31

$30.38+8.5$

9.1

$-6.25$

$30.05 \quad 19.43$

9.14

$+6.25$

$30.05 \quad 19.43$

398.09

0 
Table D.4:Input data file for Jaguara case study using frequency dependent line model

* File "Jaguara.dat".

C LINE ENERGIZATION. FROM: H.W. DOMMEL ET AL, CASE STUDIES FOR ELECTROMAGNETIC C TRANSIENTS, MAY 1983 (IATEST REVISION JAN. 1991).

JAGUARA TAQUARIL LINE ENERGIZATION with FD LINE MODEL 60 .

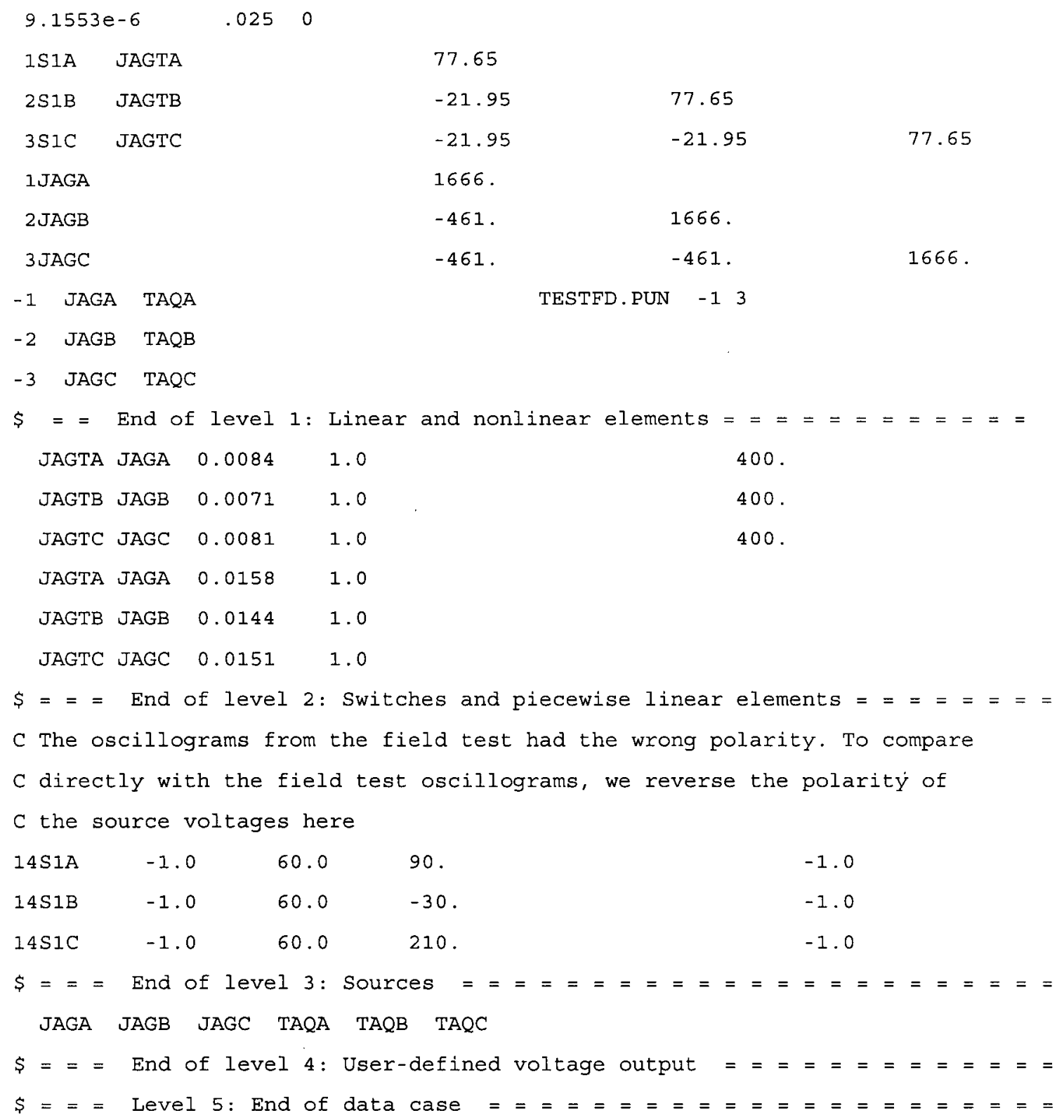


Table D.5:Error analysis in time domain

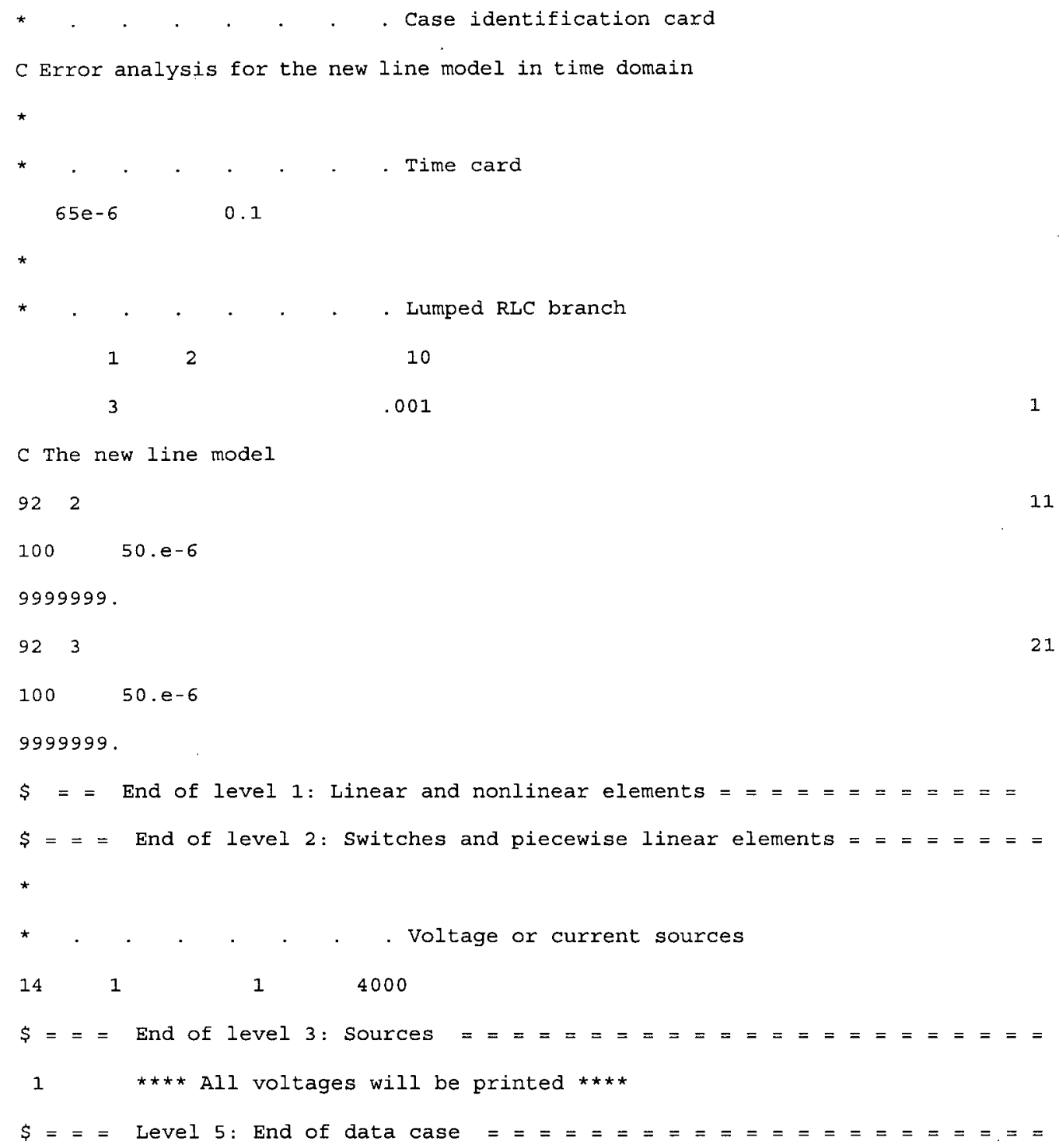

